Verification of a Six-Degree of Freedom Simulation Model

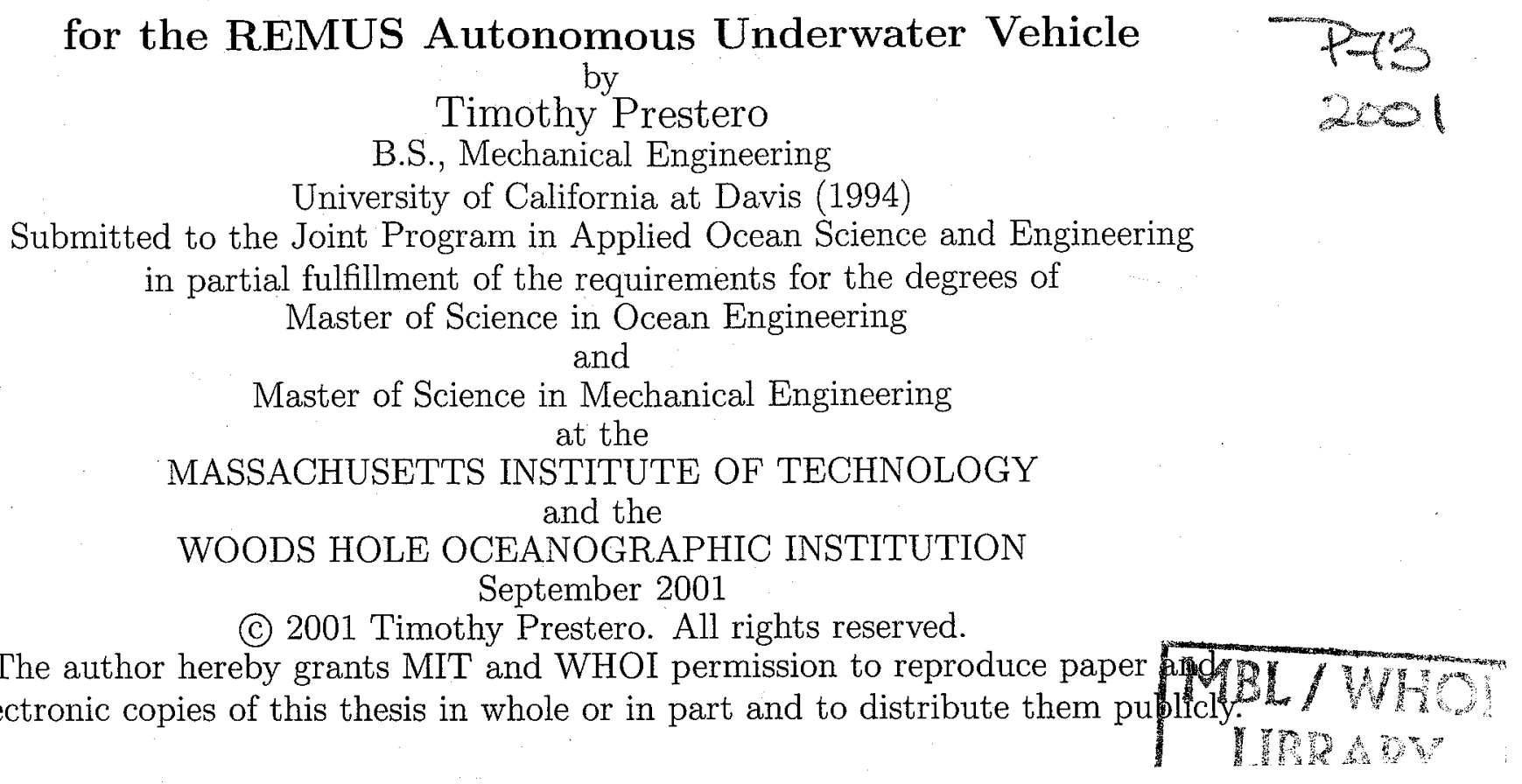




\title{
Verification of a Six-Degree of Freedom Simulation Model for the REMUS Autonomous Underwater Vehicle by Timothy Prestero
}

\author{
Submitted to the Joint Program in Applied Ocean Science and Engineering \\ on 10 August 2001, in partial fulfillment of the \\ requirements for the degrees of \\ Master of Science in Ocean Engineering \\ and \\ Master of Science in Mechanical Engineering
}

\begin{abstract}
Improving the performance of modular, low-cost autonomous underwater vehicles (AUVs) in such applications as long-range oceanographic survey, autonomous docking, and shallow-water mine countermeasures requires improving the vehicles' maneuvering precision and battery life. These goals can be achieved through the improvement of the vehicle control system. A vehicle dynamics model based on a combination of theory and empirical data would provide an efficient platform for vehicle control system development, and an alternative to the typical trial-and-error method of vehicle control system field tuning. As there exists no standard procedure for vehicle modeling in industry, the simulation of each vehicle system represents a new challenge.

Developed by von Alt and associates at the Woods Hole Oceanographic Institute, the REMUS AUV is a small, low-cost platform serving in a range of oceanographic applications. This thesis describes the development and verification of a six degree of freedom, non-linear simulation model for the REMUS vehicle, the first such model for this platform. In this model, the external forces and moments resulting from hydrostatics, hydrodynamic lift and drag, added mass, and the control inputs of the vehicle propeller and fins are all defined in terms of vehicle coefficients. This thesis describes the derivation of these coefficients in detail. The equations determining the coefficients, as well as those describing the vehicle rigid-body dynamics, are left in non-linear form to better simulate the inherently non-linear behavior of the vehicle. Simulation of the vehicle motion is achieved through numeric integration of the equations of motion. The simulator output is then checked against vehicle dynamics data collected in experiments performed at sea. The simulator is shown to accurately model the motion of the vehicle.
\end{abstract}

Thesis Supervisor: Jerome Milgram

Title: Professor of Ocean Engineering, MIT

Thesis Supervisor: Kamal Youcef-Toumi

Title: Professor of Mechanical Engineering, MIT

Thesis Supervisor: Christopher von Alt

Title: Principal Engineer, WHOI 

Candide had been wounded by some splinters of stone; he was stretched out in the street and covered with debris. He said to Pangloss: "Alas, get me a little wine and oil, I am dying."

"This earthquake is not a new thing," replied Pangloss. "The town of Lima suffered the same shocks in America last year; same causes, same effects; there is certainly a vein of sulfur underground from Lima to Lisbon."

"Nothing is more probable," said Candide, "but for the love of God, a little oil and wine."

"What do you mean, probable?" replied the philosopher. "I maintain that the matter is proved." Candide lost consciousness.

-Candide, Voltaire

Did I possess all the knowledge in the world, but had no love, how would this help me before God, who will judge me by my deeds?

-The Imitation of Christ, Thomas à Kempis 


\section{Acknowledgments}

If not for the assistance and support of the following people, this work would have been much more difficult, if not impossible to accomplish.

At MIT, I would first like to thank my advisor Prof. Jerry Milgram, for giving me a chance, for helping me to get started on such an interesting problem, and for allowing me the room to figure things out on my own. I would like to thank Prof. Kamal Youcef-Toumi for agreeing to read this thesis on top of what was already a very busy schedule. I would like to thank Prof. John Leonard for his humanity and his excellent advice. And finally, I have to thank the department administrators, Beth Tuths and Jean Sucharewicz, for their unfailing patience and courtesy in answering about a million emails from Africa.

At Woods Hole, I would like to thank Chris von Alt for his sage advice, and for his patience as I figured out how to assemble this Heath Kit. I would like to thank Ben Allen for not telling anyone that I dropped the digital camera into the tow tank. I would like to thank Roger Stokey, Tom Austin, Ned Forrester, Mike Purcell and Greg Packard for all of their help with the vehicle experiments, and for swatting their share of the green flies in Tuckerton. I would like to thank Marga McElroy for helping me navigate the WHOI bureaucracy, and I have to thank Butch Grant for inducting me into the mysteries of the circuit board and soldering iron.

I would like to thank Nuno Cruz for the excellent discussions about experimental methods, Oscar Pizarro, Chris Roman, and Fabian Tapia for solving the world's problems over dinner, and Tom Fulton and Chris Cassidy for the water-skiing lessons. And wherever he is now in Brooklyn, I have to thank Alexander Terry for that first kick in the pants.

At home, I have to thank my family for their confidence and constant support, and Sheridan for first giving me the good news. And finally, I would like to thank Elizabeth for absolutely everything.

TIMOTHY PRESTERO

Cambridge, Massachusetts 


\section{Contents}

1 Introduction 12

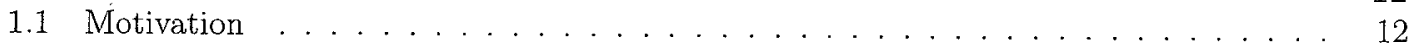

1.2 Vehicle Model Development . . . . . . . . . . . . . . . . . . . . . . 12

1.3 Research Platform . . . . . . . . . . . . . . . . . . . . . . . . . . . . . . . . . . . . . . . . . . . . . .

1.4 Model Code . . . . . . . . . . . . . . . . . . . . . . . . . . . . . . . . . . . . . . . . . . . . . . . . .

1.5 Modeling Assumptions . . . . . . . . . . . . . . . . . . . . . . . . . . . . 13

1.5.1 Environmental Assumptions . . . . . . . . . . . . . . . . . . . . . . 13

1.5.2 Vehicle/Dynamics Assumptions . . . . . . . . . . . . . . . . . . . . 13

2 The REMUS Autonomous Underwater Vehicle 14

2.1 Vehicle Profile . . . . . . . . . . . . . . . . . . . . . . . . . . . . 14

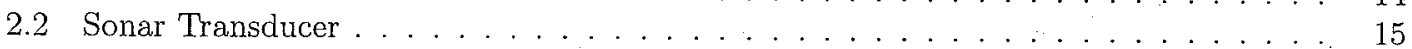

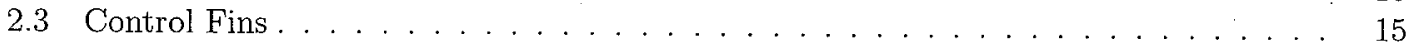

2.4 Vehicle Weight and Buoyancy . . . . . . . . . . . . . . . . . . . 15

2.5 Centers of Buoyancy and Gravity . . . . . . . . . . . . . . . . . . . . . . 17

2.6 Inertia Tensor . . . . . . . . . . . . . . . . . . . . . . . . . . . . . . . . . . . . . . . . . . . . . . . . . . . . .

2.7 Final Vehicle Profile . . . . . . . . . . . . . . . . . . . 18

3 Elements of the Governing Equations 20

3.1 Body-Fixed Vehicle Coordinate System Origin . . . . . . . . . . . . . . . . . . . 20

3.2 Vehicle Kinematics . . . . . . . . . . . . . . . . . . . . . . . . . . . . . . . . . . . . . . . . . . . . . . . . . . . . . . . . . . .

3.3 Vehicle Rigid-Body Dynamics . . . . . . . . . . . . . . . . . . . . . . . . . . . . . . . . . . . . . . . . . . . . . . . . . . . . . . . .

3.4 Vehicle Mechanics . . . . . . . . . . . . . . . . . . . . . . . . . . . . . . . . . 23

4 Coefficient Derivation $\quad 24$

4.1 Hydrostatics . . . . . . . . . . . . . . . . . . . . . . . . . . . . . 24

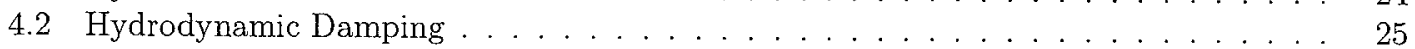

4.2 .1 Axial Drag . . . . . . . . . . . . . . . . . . . . . . . . . . . . . . . . . . . . . . . . . . . . . .

4.2 .2 Crossflow Drag . . . . . . . . . . . . . . . . . . . . . . . . . . . . . . . . . . . . . . . . . . . . . .

4.2 .3 Rolling Drag . . . . . . . . . . . . . . . . . . . . . . . . . . . . . . . . . . . . . . . . . . . . .

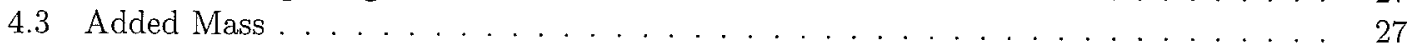

4.3 .1 Axial Added Mass . . . . . . . . . . . . . . . . . . . . . . . . . . . . . . . . . . . . . . . . . . . . . .

4.3 .2 Crossflow Added Mass . . . . . . . . . . . . . . . . . . . . . . . . . . . . . . . . . . . . . . . . .

4.3 .3 Rolling Added Mass . . . . . . . . . . . . . . . . . . . . . . . . . . . . . . . . . 29

4.3 .4 Added Mass Cross-terms . . . . . . . . . . . . . . . . . . . . . . . . . . . . . . . . . . . . . . .

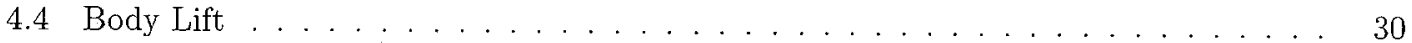

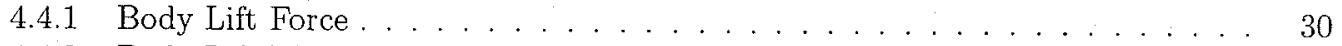

4.4 .2 Body Lift Moment . . . . . . . . . . . . . . . . . . . . . . . . . . . . . . . . . . . . . . . . . . . 31

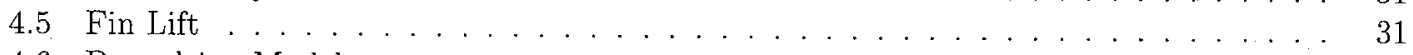

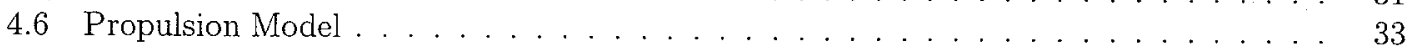


4.6 .1 Propeller Thrust . . . . . . . . . . . . . . . . . . . . . . . 33

4.6 .2 Propeller Torque . . . . . . . . . . . . . . . . . . . . . . . . . . . . . . . . . . . . . . . . . . . . .

4.7 Combined Terms . . . . . . . . . . . . . . . . . . . . . . . . . . . . . . . . . . . . . . . . . . . . . . . .

4.8 Total Vehicle Forces and Moments . . . . . . . . . . . . . . . . . . . . . . 34

5 Vehicle Tow Tank Experiments $\quad 37$

5.1 Motivation . . . . . . . . . . . . . . . . . . . . . . 37

5.2 Laboratory Facilities and Equipment . . . . . . . . . . . . . . . . . . . . . . . . . . . . . . . . . . . .

5.2 .1 Flexural Mount . . . . . . . . . . . . . . . . . . . . . . . . . . . . . . . . . . . .

5.2 .2 Tow Tank Carriage . . . . . . . . . . . . . . . . . . . . 38

5.3 Drag Test Experimental Procedure . . . . . . . . . . . . . . . . . . . . . . 41

5.3 .1 Instrument Calibration . . . . . . . . . . . . . . . . . . . 41

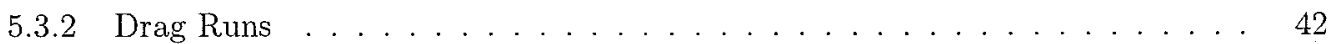

5.3 .3 Signal Processing . . . . . . . . . . . . . . . . . . . . . . . . . . . . 42

5.4 Experimental Results . . . . . . . . . . . . . . . . . . . . . . . . . . . . . . . . . . . . . . . . . . . . . . .

5.5 Component-Based Drag Model . . . . . . . . . . . . . . . . . . . . . 43

6 Vehicle Simulation $\quad \mathbf{4 5}$

6.1 Combined Nonlinear Equations of Motion . . . . . . . . . . . . . . . . . . . 45

6.2 Numerical Integration of the Equations of Motion . . . . . . . . . . . . . . . . . . . 47

6.2 .1 Euler's Method . . . . . . . . . . . . . . . . . . . . . 47

6.2 .2 Improved Euler's Method . . . . . . . . . . . . . . . . . . . 47

6.2 .3 Runge-Kutta Method . . . . . . . . . . . . . . . . . . . . . 48

6.3 Computer Simulation . . . . . . . . . . . . . . . . . . . . . . 48

7 Field Experiments $\quad 49$

7.1 Motivation . . . . . . . . . . . . . . . . . . . . . . . . . 49

7.2 Measured States . . . . . . . . . . . . . . . . . . . . . . 49

7.3 Vehicle Sensors . . . . . . . . . . . . . . . . . . . . . . . . . . 50

7.3 .1 Heading: Magnetic Compass . . . . . . . . . . . . . . . . . . . . 50

7.3 .2 Yaw Rate: Tuning Fork Gyro . . . . . . . . . . . . . . . . . . . . . 50

7.3 .3 Attitude: Tilt Sensor. . . . . . . . . . . . . . . . . . 50

7.3 .4 Depth: Pressure Sensor . . . . . . . . . . . . . . . . . . 51

7.4 Experimental Procedure . . . . . . . . . . . . . . . . . . . . . . . 51

7.4 .1 Pre-launch Check List . . . . . . . . . . . . . . . . . . . . . . 51

7.4 .2 Trim and Ballast Check . . . . . . . . . . . . . . . . . . 51

7.4 .3 Vehicle Mission Programming . . . . . . . . . . . . . . . . . . . 51

7.4 .4 Compass Calibration . . . . . . . . . . . . . . . . . . . . . 53

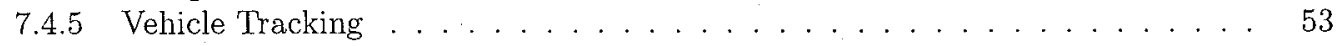

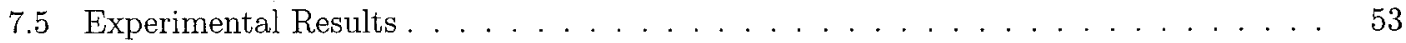

7.5 .1 Horizontal-Plane Dynamics . . . . . . . . . . . . . . . . . . . . . . . 53

7.5 .2 Vertical-Plane Dynamics . . . . . . . . . . . . . . . 55

8 Comparisons of Simulator Output and Experimental Data $\quad 60$

8.1 Model Preparation . . . . . . . . . . . . . . . . . . . . . . . 60

8.1 Initial Conditions . . . . . . . . . . . . . . . . . . . . . 60

8.1 .2 Coefficient Adjustments . . . . . . . . . . . . . . . . . . . . 60 60

8.2 Uncertainties in Model Comparison . . . . . . . . . . . . . . . . . . . . . . . . 61

8.3 Horizontal Plane Dynamics . . . . . . . . . . . . . . . . . . . . . . 61

8.4 Vertical Plane Dynamics . . . . . . . . . . . . . . . . . . . . . . . 6 61

8.4 Vehicle Pitching $\mathrm{Up} \ldots \ldots \ldots \ldots$. . . . . . . . . . . . . . . 67

8.4 .2 Vehicle Pitching Down . . . . . . . . . . . . . . . . . . . . . . . 67 
9 Linearized Depth Plane Model and Controller $\quad 78$

9.1 Linearizing the Vehicle Equations of Motion . . . . . . . . . . . . . . . . . . . . . . 78

9.1.1 Vehicle Kinematics . . . . . . . . . . . . . . . . . . . . . . . . 78

9.1 .2 Vehicle Rigid-Body Dynamics . . . . . . . . . . . . . . . . . . . 79

9.1 .3 Vehicle Mechanics . . . . . . . . . . . . . . . . . . . . 79

9.2 Linearized Coefficient Derivation . . . . . . . . . . . . . . . . . . . . . . . . 80

9.2 .1 Hydrostatics ... . . . . . . . . . . . . . . . . . . . . . . . . . . . . . . . . . . . . . . . . . .

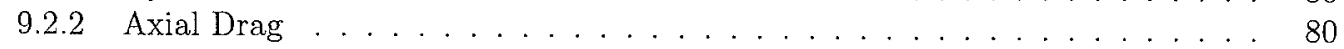

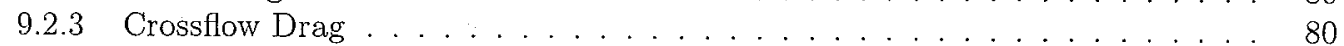

9.2 .4 Added Mass . . . . . . . . . . . . . . . . . . . . . . . . . . . . . . . . . . . . . . . . . . . . . . . . . . . . . . . . . . . . . .

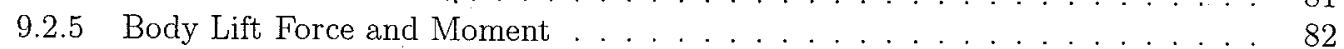

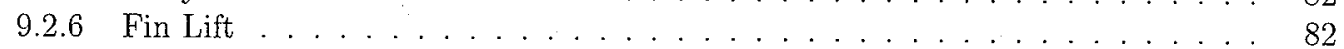

9.2 .7 Combined Terms . . . . . . . . . . . . . . . . . . . . . . . . . . . . . . . . . . . . . . . . . . . . . . . . .

9.2 .8 Linearized Coefficients . . . . . . . . . . . . . . . . . . . . . . . . . . . 83

9.3 Linearized Equations of Motion . . . . . . . . . . . . . . . . . . . . . . . . . . . . 84

9.3.1 Equations of Motion ........................ . . . . . 84

9.3 .2 Four-term State Vector . . . . . . . . . . . . . . . . . . . . . . 85

9.3.3 Three-term State Vector . . . . . . . . . . . . . . . . . . . . . . . . . . 86

9.4 Control System Design . . . . . . . . . . . . . . . . . . . . . . . . 86

9.4.1 Vehicle Transfer Functions . . . . . . . . . . . . . . . . . . . 87

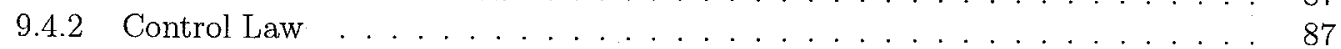

9.4 .3 Controller Design Procedure . . . . . . . . . . . . . . . . . . . . . 87

9.4 .4 Pitch Loop Controller Gains . . . . . . . . . . . . . . . . . . . . . . . . . . . . . 89

9.4.5 Depth Loop Controller Gains . . . . . . . . . . . . . . . . . . . . . . . . . . . . . . 89

9.5 Real-World Phenomena . . . . . . . . . . . . . . . . . . . . . . . . . . . . . . . . . . . 89

9.6 Controller Implementation . . . . . . . . . . . . . . . . . . . . . . . . . . . . . . . 93

10 Conclusion
99

10.1 Expanded Tow Tank Measurements . . . . . . . . . . . . . . . . . . . . . . . . . 99

10.2 Future Experiments at Sea . . . . . . . . . . . . . . . . . . . . . 100

10.2 .1 Improved Vehicle Instrumentation . . . . . . . . . . . . . . . . . . . . . . . . . . . . . . . . . . . . .

10.2 .2 Measurement of Vehicle Parameters . . . . . . . . . . . . . . . . . . . . . . . . . . . . . . . . . . 100

10.2 .3 Isolation of Vehicle Motion . . . . . . . . . . . . . . . . . . . . . . . . . . . . . . . . . . . . . . . . .

10.3 Controller-Based Model Comparison . . . . . . . . . . . . . . . . . . . . . . . . 100

10.4 Vehicle Sensor Model . . . . . . . . . . . . . . . . . . . . . . . . . . . . . . 101

10.5 Improved Coefficient-Based Model . . . . . . . . . . . . . . . . . . . . . . . . . 101

A Tables of Parameters $\quad 102$

B Tables of Combined Non-Linear Coefficients 104

C Tables of Non-Linear Coefficients by Type 106

D Tables of Linearized Model Parameters 112

E MATLAB Code $\quad 114$

E.1 Vehicle Simulation . . . . . . . . . . . . . . . . . . . . . . 114

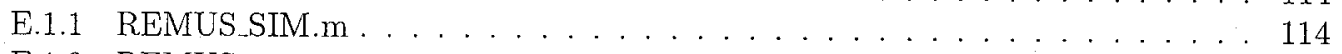

E.1.2 REMUS.m . . . . . . . . . . . . . . . . . . . 117

F Example REMUS Mission File $\quad 120$

F.1 REMUS Mission Code . . . . . . . . . . . . . . . . . . . . . . . . . . . . . . . 120 


\section{List of Figures}

2-1 Myring Profile . . . . . . . . . . . . . . . . . . . . . . 15

2-2 REMUS Low-Frequency Sonar Transducer (XZ-plane) $\ldots \ldots \ldots \ldots \ldots \ldots$

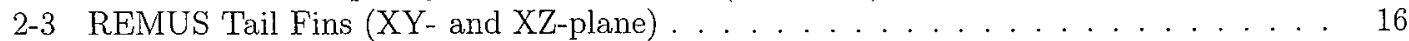

2-4 STD REMUS Profile (XZ-plane) . . . . . . . . . . . . . . . . . . . . . . . . . . . . . 19

2-5 The REMUS Autonomous Underwater Vehicle . . . . . . . . . . . . . . . . . . 19

3-1 REMUS Body-Fixed and Inertial Coordinate Systems $\ldots \ldots \ldots \ldots$

4-1 Effective Rudder Angle of Attack . . . . . . . . . . . . . . . . . . . . . . 32

4-2 Effective Stern Plane Angle of Attack . . . . . . . . . . . . . . . . 32

5-1 URI Tow Tank Layout . . . . . . . . . . . . . . . . . . . . . . . . . . . 38

$5-2$ URI Tow Tank . . . . . . . . . . . . . . . . . . . . . . . . . 39

$5-3$ Carriage Setup and Vehicle Mounting . . . . . . . . . . . . . . . . 40

$5-4$ URI Tow Tank Carriage . . . . . . . . . . . . . . . . . . . . . 40

5-5 URI Tow Tank Carriage . . . . . . . . . . . . . . . . . . . . . . 41

$5-6 \quad$ Unfiltered and Filtered Drag Data . . . . . . . . . . . . . . . . . . 43

5-7 Forward Speed vs. Vehicle Axial and Lateral Drag . . . . . . . . . . . . . . . . 44

7-1 Vehicle Experiments at the Rutgers Marine Field Station . . . . . . . . . . . 49

$7-2$ REMUS Pre-Launch Checklist (Page One) . . . . . . . . . . . . . . . 52

$7-3$ REMUS Mission Data: Crash Plot . . . . . . . . . . . . . . . . . . . . 54

$7-4$ Vehicle Experiments at WHOI . . . . . . . . . . . . . . . 55

$7-5$ The REMUS Ranger . . . . . . . . . . . . . . . . . . . . . 55

7-6 REMUS Mission Data: Closed-Loop Control . . . . . . . . . . . . . . . 56

$7-7$ REMUS Mission Data: Rudder . . . . . . . . . . . . . . . . . . . . . . 57

$7-8$ REMUS Mission Data: Pitching $\mathrm{Up} \ldots \ldots \ldots \ldots \ldots \ldots$

$7-9$ REMUS Mission Data: Pitching Down . . . . . . . . . . . . . . . . . . 59

8-1 Horizontal Plane Simulation: Linear . . . . . . . . . . . . . . . . . . . 62

$8-2$ Horizontal Plane Simulation: Angular . . . . . . . . . . . . . . . . . 63

8-3 Horizontal Plane Simulation: Forces and Moments . . . . . . . . . . . . . . . . 64

8-4 Horizontal Plane Simulation: Vehicle Trajectory . . . . . . . . . . . . . . 65

8-5 Horizontal Plane Simulation: Model Comparison . . . . . . . . . . . . . . . 66

$8-6$ Vertical Plane Simulation: Linear . . . . . . . . . . . . . . . . . . 68

8-7 Vertical Plane Simulation: Angular . . . . . . . . . . . . . . . . . . . . 69

8-8 Vertical Plane Simulation: Forces and Moments . . . . . . . . . . . . . . . . . 70

8-9 Vertical Plane Simulation: Vehicle Trajectory . . . . . . . . . . . . . . . . . 71

8-10 Vertical Plane Simulation: Model Comparison . . . . . . . . . . . . . . . . . 72

8-11 Vertical Plane Simulation: Linear . . . . . . . . . . . . . . . . . . . . . . 73

$8-12$ Vertical Plane Simulation: Angular . . . . . . . . . . . . . . . . . . 74 
8-13 Vertical Plane Simulation: Forces and Moments . . . . . . . . . . . . . . . . . . . . . 75

8-14 Vertical Plane Simulation: Vehicle Trajectory . . . . . . . . . . . . . . . . . . . . . . 76

8-15 Vertical Plane Simulation: Model Comparison . . . . . . . . . . . . . . . . . . . . 77

9-1 Perturbation Velocity Linearization . . . . . . . . . . . . . . . . . . . . . . . . 8 81

9-2 Depth-Plane Control System Block Diagram . . . . . . . . . . . . . . . . . . . . . . . 88

$9-3 \quad G_{\theta}$ Pole-Zero Plot . . . . . . . . . . . . . . . . . . . . . . . . . . . . . . . . . 90

$9-4 G_{\theta}$ Open-Loop Step Response . . . . . . . . . . . . . . . . . . . . . . . 90

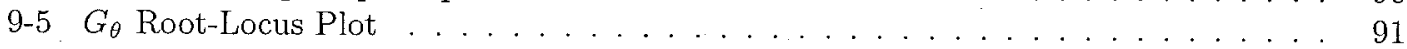

$9-6 \quad G_{\theta}$ Closed-Loop Step Response . . . . . . . . . . . . . . . . . . . . . . . . . . . . 91

$9-7 \quad G_{z} * H_{\theta}$ Pole-Zero Plot . . . . . . . . . . . . . . . . . . . . . . . . . . . . . 92

$9-8 \quad G_{z} * H_{\theta}$ Root-Locus Plot . . . . . . . . . . . . . . . . . . . . . . . . 92

$9-9 \quad G_{z} * H_{\theta}$ Closed-Loop Step Response . . . . . . . . . . . . . . . . . . . . . . . 94

9-10 Modified Depth Plane Control System Block Diagram . . . . . . . . . . . . . . . . . 94

9-11 Vehicle Simulation: Case One . . . . . . . . . . . . . . . . . . . . . . . . 95

9-12 Vehicle Simulation: Case Two . . . . . . . . . . . . . . . . . . . . . . . . . . . . . . . . . . . . . . . . .

9-13 Vehicle Simulation: Case Three . . . . . . . . . . . . . . . . . . . . . . . . 97

9-14 Vehicle Simulation: Case Four . . . . . . . . . . . . . . . . . . . . . . . . . . . . . . . 98

10-1 Forces on the vehicle at an angle of attack . . . . . . . . . . . . . . . . . . . . . 99

10-2 Vehicle performance limits as a function of depth and sea state . . . . . . . . . . 101 


\section{List of Tables}

2.1 Myring Parameters for STD REMUS . . . . . . . . . . . . . . . . . . . . . . . . . . . . . . . . . . . . . .

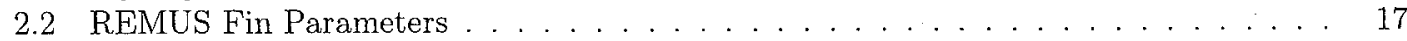

2.3 STD REMUS Weight and Buoyancy . . . . . . . . . . . . . . . . . . . . 17

2.4 STD REMUS Center of Buoyancy . . . . . . . . . . . . . . . . . . . . . . . 17

2.5 STD REMUS Center of Gravity . . . . . . . . . . . . . . . . . . . . . . . . . . . . 18

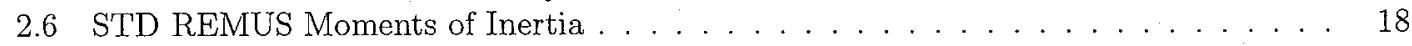

2.7 STD REMUS Hull Parameters . . . . . . . . . . . . . . . . . . . . . . . 18

4.1 Axial Added Mass Parameters $\alpha$ and $\beta$. . . . . . . . . . . . . . . . . . . . 28

4.2 STD REMUS Non-Linear Maneuvering Coefficients: Forces . . . . . . . . . . . . . . . . . . 35

4.3 STD REMUS Non-Linear Maneuvering Coefficients: Moments . . . . . . . . . . . . 36

5.1 REMUS Drag Runs . . . . . . . . . . . . . . . . . . . . . . . . . . . . . . . 42

5.2 REMUS Component-Based Drag Analysis - Standard Vehicle . . . . . . . . . . . . . 44

5.3 REMUS Component-Based Drag Analysis - Sonar Vehicle . . . . . . . . . . . . . . 44

7.1 Vehicle Field Experiments . . . . . . . . . . . . . . . . . . . 51

8.1 REMUS Simulator Initial Conditions . . . . . . . . . . . . . . . . . . . . . 60

8.2 Vehicle Coefficient Adjustment Factors . . . . . . . . . . . . . . . . . . . . . 61

9.1 Linearized Velocity Parameters . . . . . . . . . . . . . . . . . . . . . . . . . . . . . . 81

9.2 Combined Linearized Coefficients . . . . . . . . . . . . . . . . . . . . . . . . . . . . . 84

9.3 Linearized Maneuvering Coefficients . . . . . . . . . . . . . . . . . . . . . 85

9.4 Percent Overshoot and Damping Ratio . . . . . . . . . . . . . . . . . . . . . 88

A.1 STD REMUS Hull Parameters . . . . . . . . . . . . . . . . . . . . . . . . . . . . . 102

A.2 Hull Coordinates for Limits of Integration . . . . . . . . . . . . . . . . . . . . . . 102

A.3 STD REMUS Center of Buoyancy . . . . . . . . . . . . . . . . . . . . . . . . . . . . . . . . . . . . . . . . . . . . . .

A.4 STD REMUS Center of Gravity . . . . . . . . . . . . . . . . . . . . . . . . 103

A.5 REMUS Fin Parameters . . . . . . . . . . . . . . . . . . . . . . 103

B.1 Non-Linear Force Coefficients . . . . . . . . . . . . . . . . . . . . . . . . . . . . . . 104

B.2 Non-Linear Moment Coefficients . . . . . . . . . . . . . . . . . . . . 105

C.1 Axial Drag Coefficient . . . . . . . . . . . . . . . . . . . . . 106

C.2 Crossflow Drag Coefficients . . . . . . . . . . . . . . . . . . . . . 106

C.3 Rolling Resistance Coefficient . . . . . . . . . . . . . . . . . . . . . . 106

C.4 Body Lift and Moment Coefficients . . . . . . . . . . . . . . . . . . . . . . . . . . . 107

C.5 Added Mass Coefficients . . . . . . . . . . . . . . . . . . . . . . . . . . . . 107

C.6 Added Mass Force Cross-term Coefficients . . . . . . . . . . . . . . . . . . . . . . . 108 
C.7 Added Mass K-Moment Cross-term Coefficients . . . . . . . . . . . . . . . . . . . 109

C.8 Added Mass M-, N-Moment Cross-term Coefficients . . . . . . . . . . . . . . . 110

C.9 Propeller Terms . . . . . . . . . . . . . . . . . . . . . . . . . . . . . . . . 110

C.10 Control Fin Coefficients . . . . . . . . . . . . . . . . . . 111

D.1 Linearized Combined Coefficients . . . . . . . . . . . . . . . . . . . . . . 112

D.2 Linearized Maneuvering Coefficients . . . . . . . . . . . . . . . . . . 113 


\section{Chapter 1}

\section{Introduction}

\subsection{Motivation}

Improving the performance of modular, low-cost autonomous underwater vehicles (AUVs) in such applications as long-range oceanographic survey, autonomous docking, and shallow-water mine countermeasures requires improving the vehicles' maneuvering precision and battery life. These goals can be achieved through the improvement of the vehicle control system. A vehicle dynamics model based on a combination of theory and empirical data would provide an efficient platform for vehicle control system development, and an alternative to the typical trial-and-error method of vehicle control system field tuning. As there exists no standard procedure for vehicle modeling in industry, the simulation of each vehicle system represents a new challenge.

\subsection{Vehicle Model Development}

This thesis describes the development and verification of a simulation model for the motion of the REMUS vehicle in six degrees of freedom. In this model, the external forces and moments resulting from hydrostatics, hydrodynamic lift and drag, added mass, and the control inputs of the vehicle propeller and fins are all defined in terms of vehicle coefficients.

This thesis describes the derivation of these coefficients in detail, and describes the experimental measurement of the vehicle axial drag.

The equations determining the coefficients, as well as those describing the vehicle rigid-body dynamics, are left in non-linear form to better simulate the inherently non-linear behavior of the vehicle. Simulation of the vehicle motion is achieved through numeric integration of the equations of motion. The simulator output is then checked against open-loop data collected in the field. This field data measured the vehicle response to step changes in control fin angle. The simulator is shown to accurately model the vehicle motion in six degrees of freedom.

To demonstrate the intended application of this work, this thesis demonstrates the use of a linearized version of the vehicle model to develop a vehicle depth-plane control system.

In closing, this thesis discusses plans for further experimental verification of the vehicle coefficients, including tow tank lift and drag measurements, and precision inertial measurements of the vehicle open-water motion and sensor dynamics.

\subsection{Research Platform}

The platform for this research is the REMUS AUV, developed by von Alt and associates at the Oceanographic Systems Laboratory at the Woods Hole Oceanographic Institution [31]. REMUS 
(Remote Environmental Monitoring Unit) is a low-cost, modular vehicle with applications in autonomous docking, long-range oceanographic survey, and shallow-water mine reconnaissance [30]. See Chapter 2 for the specifications of the REMUS vehicle.

REMUS currently uses a field-tuned PID controller; previous attempts to apply more advanced controllers to REMUS have been hampered by the lack of a mathematical model to describe the vehicle dynamics.

\subsection{Model Code}

The author developed the simulator code using MATLAB. Although MATLAB runs slowly compared to other compilers, the program greatly facilitates data visualization. In developing the code, the author did not use any MATLAB-specific functions, so exporting the model code to another, faster language for controller development will be easy.

\subsection{Modeling Assumptions}

In order to simplify the challenge of modeling an autonomous underwater vehicle, it is necessary to make some assumptions on which to base the model development.

\subsubsection{Environmental Assumptions}

The author made the following assumptions about the vehicle with respect to its environment:

- The vehicle is deeply submerged in a homogeneous, unbounded fluid. In other words, the vehicle is located far from free surface (no surface effects, i.e. no sea wave or vehicle wave-making loads), walls and bottom.

- The vehicle does not experience memory effects. The simulator neglects the effects of the vehicle passing through its own wake.

- The vehicle does not experience underwater currents.

\subsubsection{Vehicle/Dynamics Assumptions}

The author made the following assumptions about the vehicle itself:

- The vehicle is a rigid body of constant mass. In other words, the vehicle mass and mass distribution do not change during operation.

- Control surface assumptions: We assume that the control fins do not stall regardless of angle of attack. We also assume an instantaneous fin response, meaning that that vehicle actuator time response is small in comparison with the vehicle attitude time response.

- Thruster assumptions: We will be using an extremely simple propulsion model, which treats the vehicle propeller as a source of constant thrust and torque.

- There exist no significant vehicle dynamics faster than $45 \mathrm{~Hz}$ (the modeling time step). 


\section{Chapter 2}

\section{The REMUS Autonomous Underwater Vehicle}

In order to calculate the vehicle coefficients, we must first define the profile of the vehicle, determine its mass, mass distribution, and buoyancy, and finally identify the necessary control fin parameters.

\subsection{Vehicle Profile}

The hull shape of the REMUS vehicle is based on the Myring hull profile equations [22], which describe a body contour with minimal drag coefficient for a given fineness ratio (body length/maximum diameter). These equations have been modified so as to be defined in terms of the following parameters:

- $a, b$, and $c$, the full lengths of the nose-section, constant-radius center-section, and tail-section of the vehicle, respectively

- $n$, an exponential parameter which can be varied to give different body shapes.

- $2 \theta$, the included angle at the tip of the tail

- $d$, the maximum body diameter

These equations assume an origin at the nose of the vehicle.

Nose shape is given by the modified semi-elliptical radius distribution

$$
r(\Xi)=\frac{1}{2} d\left[1-\left(\frac{\Xi+a_{\text {offset }}-a}{a}\right)^{2}\right]^{\frac{1}{n}}
$$

where $r$ is the radius of the vehicle hull measured normal to the centerline, $\Xi$ is the axial position along the centerline, and $a_{\text {offset }}$ is the missing length of the vehicle nose. See Figure 2-1 for a diagram of these parameters, and see Figure 3-1 for a diagram of the vehicle coordinate system.

Tail shape is given by the equation

$$
r(\Xi)=\frac{1}{2} d-\left[\frac{3 d}{2 c^{2}}-\frac{\tan \theta}{c}\right](\Xi-l)^{2}+\left[\frac{d}{c^{3}}-\frac{\tan \theta}{c^{2}}\right]\left(\Xi-l_{f}\right)^{3}
$$

where the forward body length

$$
l_{f}=a+b-a_{\mathrm{offset}}
$$


and again, $r$ is the vehicle hull radius and $\Xi$ is the axial position along the centerline. Note in Figure 2-1 that $c_{\text {offset }}$ is the missing length of the vehicle tail, where $c$ is the full Myring tail length.

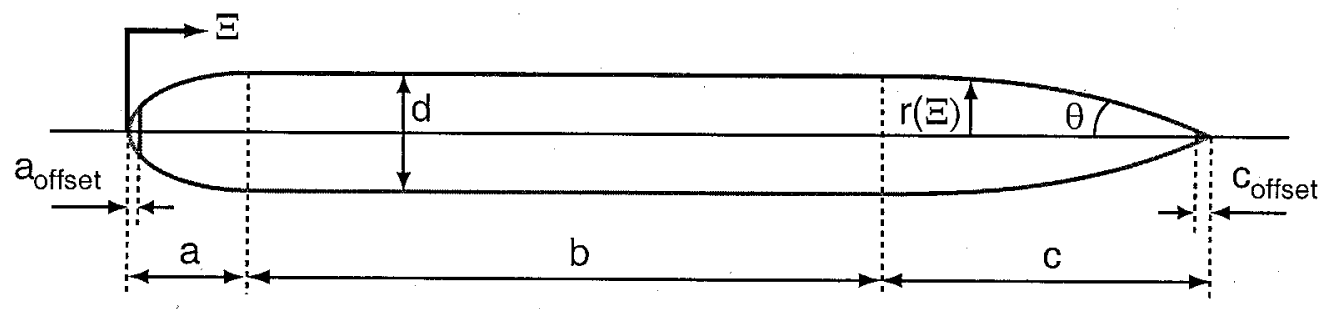

Figure 2-1: Myring Profile: vehicle hull radius as a function of axial position

For reference, Myring [22, p. 189] assumes a total body length of 100 units, and classifies body types by a code of the form $a / b / n / \theta / \frac{1}{2} d$, where $\theta$ is given in radians. REMUS is based on the Myring B hull contour, which is given by the code 15/55/1.25/0.4363/5. Table 2.1 gives the dimensionalized Myring parameters.

Table 2.1: Myring Parameters for STD REMUS

\begin{tabular}{cccl}
\hline \hline Parameter & Value & Units & Description \\
\hline$a$ & $+1.91 \mathrm{e}-001$ & $\mathrm{~m}$ & Nose Length \\
$a_{\text {offset }}$ & $+1.65 \mathrm{e}-002$ & $\mathrm{~m}$ & Nose Offset \\
$b$ & $+6.54 \mathrm{e}-001$ & $\mathrm{~m}$ & Midbody Length \\
$c$ & $+5.41 \mathrm{e}-001$ & $\mathrm{~m}$ & Tail Length \\
$c_{\text {offset }}$ & $+3.68 \mathrm{e}-002$ & $\mathrm{~m}$ & Tail Offset \\
$n$ & +2.00 & $\mathrm{n} / \mathrm{a}$ & Exponential Coefficient \\
$\theta$ & $+4.36 \mathrm{e}-001$ & radians & Included Tail Angle \\
$d$ & $+1.91 \mathrm{e}-001$ & $\mathrm{~m}$ & Maximum Hull Diameter \\
$l_{f}$ & $+8.28 \mathrm{e}-001$ & $\mathrm{~m}$ & Vehicle Forward Length \\
$l$ & $+1.33 \mathrm{e}+000$ & $\mathrm{~m}$ & Vehicle Total Length \\
\hline \hline
\end{tabular}

\subsection{Sonar Transducer}

The REMUS vehicle is equipped with a forward sonar transducer, which is a cylinder $10.1 \mathrm{~cm}$ (4.0 in) diameter. The remaining transducer dimensions are given in Figure 2-2.

\subsection{Control Fins}

The REMUS vehicle is equipped with four identical control fins, mounted in a cruciform pattern near the aft end of the hull. These fins have a NACA 0012 cross-section; their remaining dimensions are given in Figure 2-3. The relevant fin parameters are given in Table 2.2.

\subsection{Vehicle Weight and Buoyancy}

The weight of the REMUS vehicle can change between missions, depending on the type of batteries used in the vehicle and the amount of ballast added. REMUS is typically ballasted with around 


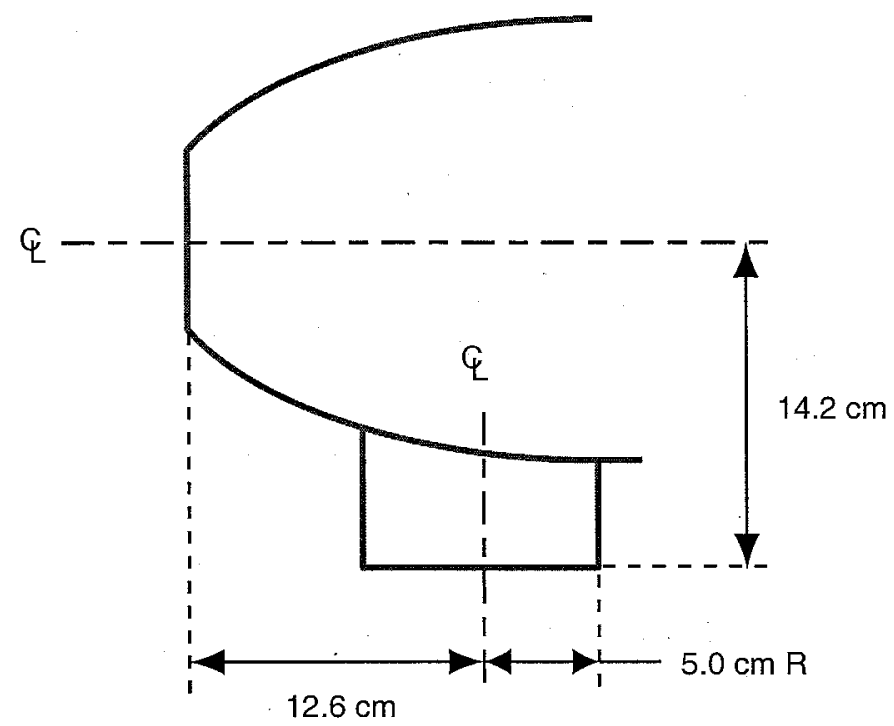

Figure 2-2: REMUS Low-Frequency Sonar Transducer (XZ-plane)

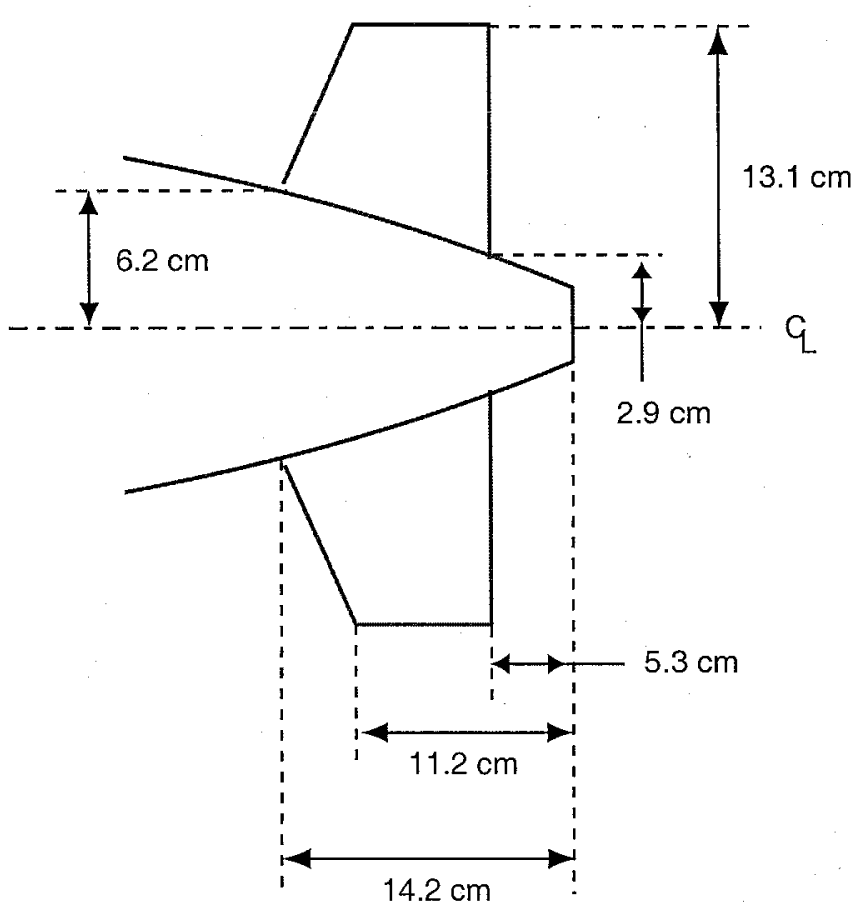

Figure 2-3: REMUS Tail Fins (XY-and XZ-plane) 
Table 2.2: REMUS Fin Parameters

\begin{tabular}{cccl}
\hline \hline Parameter & Value & Units & Description \\
\hline$S_{\text {fin }}$ & $+6.65 e-003$ & $\mathrm{~m}^{2}$ & Planform Area \\
$b_{\text {fin }}$ & $+8.57 e-002$ & $\mathrm{~m}$ & Span \\
$x_{\text {finpost }}$ & $-6.38 \mathrm{e}-001$ & $\mathrm{~m}$ & Moment Arm wrt Vehicle Origin at CB \\
$\delta_{\max }$ & $+1.36 e+001$ & $\mathrm{deg}$ & Maximum Fin Angle \\
$a_{\text {fin }}$ & $+5.14 \mathrm{e}+000$ & $\mathrm{~m}$ & Max Fin Height Above Centerline \\
$c_{\text {mean }}$ & $+7.47 \mathrm{e}-002$ & $\mathrm{~m}$ & Mean Chord Length \\
$t$ & $+6.54 \mathrm{e}-001$ & $\mathrm{n} / \mathrm{a}$ & Fin Taper Ratio (Whicker-Felner) \\
$c_{\mathrm{df}}$ & $+5.58 \mathrm{e}-001$ & $\mathrm{n} / \mathrm{a}$ & Fin Crossflow Drag Coefficient \\
$\mathrm{AR}_{e}$ & $+2.21 \mathrm{e}+000$ & $\mathrm{n} / \mathrm{a}$ & Effective Aspect Ratio \\
$\bar{a}$ & $+9.00 \mathrm{e}-001$ & $\mathrm{n} / \mathrm{a}$ & Lift Slope Parameter \\
$c_{L \alpha}$ & $+3.12 \mathrm{e}+000$ & $\mathrm{n} / \mathrm{a}$ & Fin Lift Slope \\
\hline \hline
\end{tabular}

1.5 pounds of buoyancy, so that it will eventually float to the surface in the event of a computer or power failure. Typical values for the vehicle weight and buoyancy are given in Table 2.3 .

Table 2.3: Vehicle Weight and Buoyancy

\begin{tabular}{ccc}
\hline \hline Parameter & Value & Units \\
\hline$W$ & $+2.99 \mathrm{e}+002$ & $\mathrm{~N}$ \\
$B$ & $+3.06 \mathrm{e}+002$ & $\mathrm{~N}$ \\
\hline \hline
\end{tabular}

\subsection{Centers of Buoyancy and Gravity}

For a given REMUS vehicle during field operations, the center of buoyancy stays roughly constant as there are rarely any changes made to the exterior of the hull. The vehicle center of gravity, on the other hand, can vary, as between missions it is often necessary to change the vehicle battery packs and re-ballast the vehicle.

The average values are given in Tables 2.4 and 2.5 .

Table 2.4: Center of Buoyancy wrt Origin at Vehicle Nose

\begin{tabular}{ccc}
\hline Parameter & Value & Units \\
\hline$x_{\mathrm{cb}}$ & $-6.11 \mathrm{e}-001$ & $\mathrm{~m}$ \\
$y_{\mathrm{cb}}$ & $+0.00 \mathrm{e}+000$ & $\mathrm{~m}$ \\
$z_{\mathrm{cb}}$ & $+0.00 \mathrm{e}+000$ & $\mathrm{~m}$ \\
\hline \hline
\end{tabular}

\subsection{Inertia Tensor}

The vehicle inertia tensor is defined with respect to the body-fixed origin at the vehicle center of buoyancy. As the products of inertia $I_{x y}, I_{x z}$, and $I_{y z}$ are small compared to the moments of inertia $I_{x x}, I_{y y}$, and $I_{z z}$, we will assume that they are zero, in effect assuming that the vehicle has two axial planes of symmetry.

These values were estimated based on the vehicle weight list (a table listing the locations and weights of the various vehicle internal components). Although the changes in the vehicle center of 
Table 2.5: Center of Gravity wrt Origin at CB

\begin{tabular}{ccc}
\hline \hline Parameter & Value & Units \\
\hline$x_{\mathrm{cg}}$ & $+0.00 \mathrm{e}+000$ & $\mathrm{~m}$ \\
$y_{\mathrm{cg}}$ & $+0.00 \mathrm{e}+000$ & $\mathrm{~m}$ \\
$z_{\mathrm{cg}}$ & $+1.96 \mathrm{e}-002$ & $\mathrm{~m}$ \\
\hline \hline
\end{tabular}

gravity described above will obviously affect the vehicle moments of inertia, we will assume that these changes are small enough to be ignored. The estimated values are given in Table 2.6.

Table 2.6: Moments of Inertia wrt Origin at CB

\begin{tabular}{ccc}
\hline \hline Parameter & Value & Units \\
\hline$I_{x x}$ & $+1.77 \mathrm{e}-001$ & $\mathrm{~kg} \cdot \mathrm{m}^{2}$ \\
$I_{y y}$ & $+3.45 \mathrm{e}+000$ & $\mathrm{~kg} \cdot \mathrm{m}^{2}$ \\
$I_{z z}$ & $+3.45 \mathrm{e}+000$ & $\mathrm{~kg} \cdot \mathrm{m}^{2}$ \\
\hline
\end{tabular}

\subsection{Final Vehicle Profile}

Figure 2-4 shows the complete vehicle profile, plotted over an ellipsoid for reference. Some additional hull parameters, mostly functions of hull geometry, are given in Table 2.7.

Note that the estimates for vehicle buoyancy and longitudinal center of buoyancy are based solely on the bare hull profile, and do not account for the vehicle fins and transponder, or the flooded sections in the vehicle nosecap. The $x_{c b}$ value given in Table 2.4 and the total buoyancy $B$ given in Table 2.3 are based on experimental measurements.

Table 2.7: STD REMUS Hull Parameters

\begin{tabular}{cccl}
\hline \hline Parameter & Value & Units & Description \\
\hline$\rho$ & $+1.03 \mathrm{e}+003$ & $\mathrm{~kg} / \mathrm{m}^{3}$ & Seawater Density \\
$A_{f}$ & $+2.85 \mathrm{e}-002$ & $\mathrm{~m}^{2}$ & Hull Frontal Area \\
$A_{p}$ & $+2.26 \mathrm{e}-001$ & $\mathrm{~m}^{2}$ & Hull Projected Area (xz plane) \\
$S_{w}$ & $+7.09 \mathrm{e}-001$ & $\mathrm{~m}^{2}$ & Hull Wetted Surface Area \\
$\nabla$ & $+3.15 \mathrm{e}-002$ & $\mathrm{~m}^{3}$ & Estimated Hull Volume \\
$B_{\text {est }}$ & $+3.17 \mathrm{e}+002$ & $\mathrm{~N}$ & Estimated Hull Buoyancy \\
$x_{\mathrm{cb} \text { (est) }}$ & $+5.54 \mathrm{e}-003$ & $\mathrm{~m}$ & Est. Long. Center of Buoyancy \\
\hline \hline
\end{tabular}




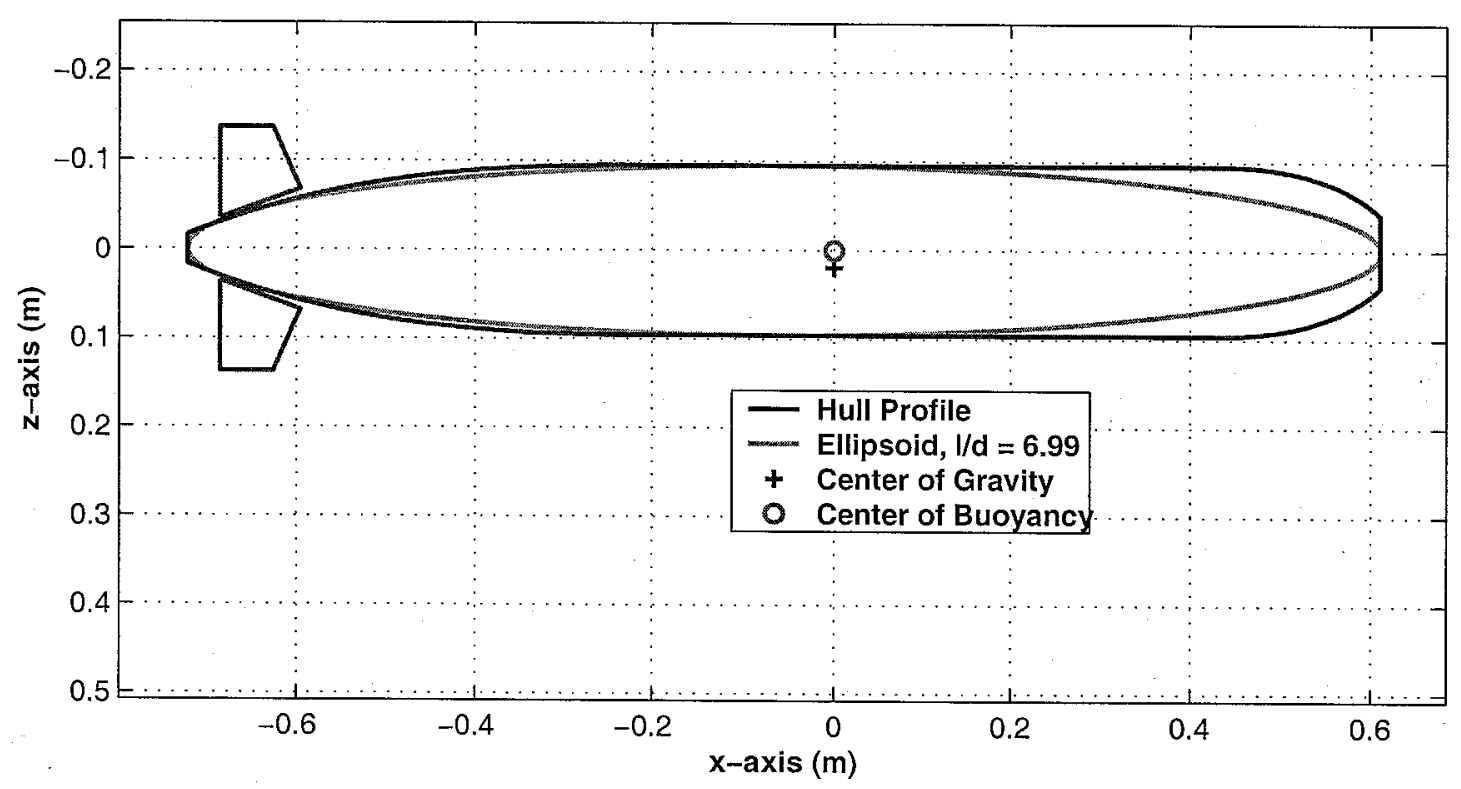

Figure 2-4: STD REMUS Profile (XZ-plane)

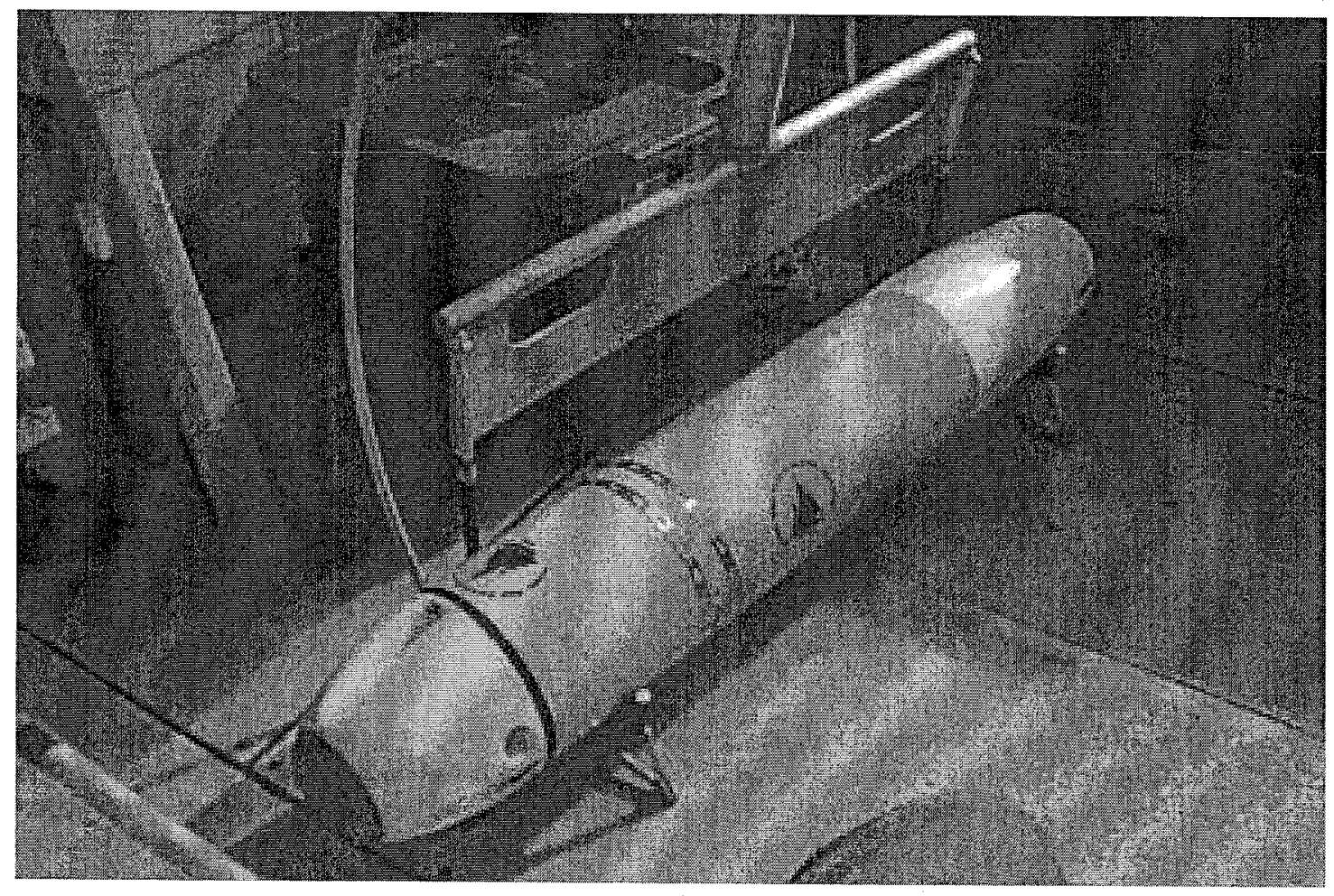

Figure 2-5: The REMUS Autonomous Underwater Vehicle 


\section{Chapter 3}

\section{Elements of the Governing Equations}

In this chapter, we define the equations governing the motion of the vehicle. These equations consist of the following elements:

- Kinematics: the geometric aspects of motion

- Rigid-body Dynamics: the vehicle inertia matrix

- Mechanics: forces and moments causing motion

These elements are addressed in the following sections.

\subsection{Body-Fixed Vehicle Coordinate System Origin}

Please note that in all future calculations, the origin of the vehicle body-fixed coordinate system is located at the vehicle center of buoyancy, as defined in Section 2.5 and illustrated in Figure 2-4.

\subsection{Vehicle Kinematics}

The motion of the body-fixed frame of reference is described relative to an inertial or earth-fixed reference frame. The general motion of the vehicle in six degrees of freedom can be described by the following vectors:

$$
\begin{array}{rlrl}
\eta_{1} & =\left[\begin{array}{lll}
x & y & z
\end{array}\right]^{T} ; & & \eta_{2}=\left[\begin{array}{lll}
\phi & \theta & \psi
\end{array}\right]^{T} \\
\boldsymbol{v}_{1} & =\left[\begin{array}{lll}
u & v & w
\end{array}\right]^{T} ; & \boldsymbol{v}_{2}=\left[\begin{array}{lll}
p & q & r
\end{array}\right]^{T} \\
\tau_{1}=\left[\begin{array}{lll}
X & Y & Z
\end{array}\right]^{T} ; & \tau_{2}=\left[\begin{array}{lll}
K & M & N
\end{array}\right]^{T}
\end{array}
$$

where $\boldsymbol{\eta}$ describes the position and orientation of the vehicle with respect to the inertial or earthfixed reference frame, $v$ the translational and rotational velocities of the vehicle with respect to the body-fixed reference frame, and $\tau$ the total forces and moments acting on the vehicle with respect to the body-fixed reference frame. See Figure 3-1 for a diagram of the vehicle coordinate system. 


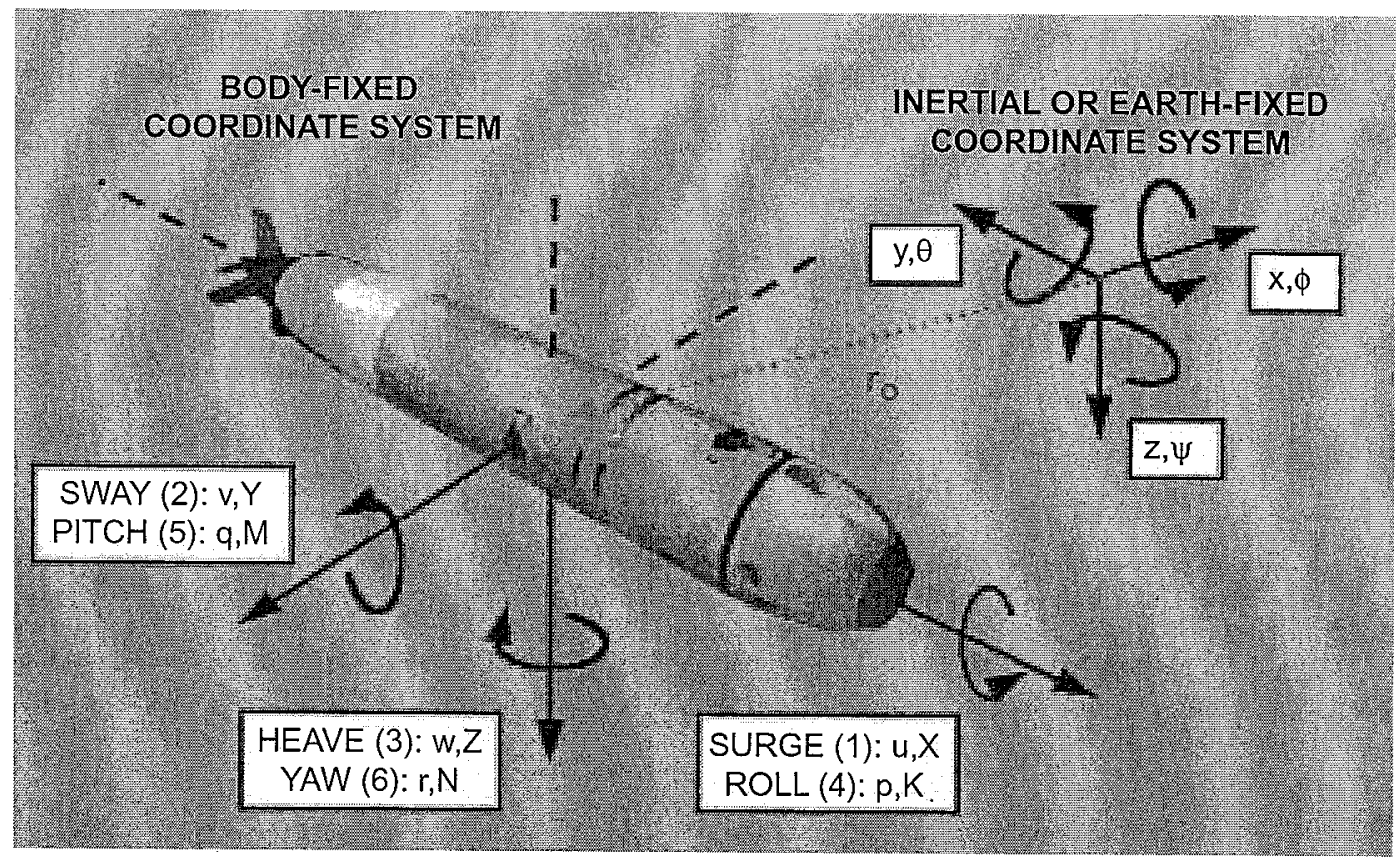

Figure 3-1: REMUS Body-Fixed and Inertial Coordinate Systems

The following coordinate transform relates translational velocities between body-fixed and inertial or earth-fixed coordinates:

$$
\left[\begin{array}{c}
\dot{x} \\
\dot{y} \\
\dot{z}
\end{array}\right]=J_{1}\left(\eta_{2}\right)\left[\begin{array}{c}
u \\
v \\
w
\end{array}\right]
$$

where

$$
J_{1}\left(\eta_{2}\right)=\left[\begin{array}{ccc}
\cos \psi \cos \theta & -\sin \psi \cos \phi+\cos \psi \sin \theta \sin \phi & \sin \psi \sin \phi+\cos \psi \sin \theta \cos \phi \\
\sin \psi \cos \theta & \cos \psi \cos \phi+\sin \psi \sin \theta \sin \phi & -\cos \psi \sin \phi+\sin \psi \sin \theta \cos \phi \\
-\sin \theta & \cos \theta \sin \phi & \cos \theta \cos \phi
\end{array}\right]
$$

Note that $J_{1}\left(\eta_{2}\right)$ is orthogonal:

$$
\left(J_{1}\left(\eta_{2}\right)\right)^{-1}=\left(J_{1}\left(\boldsymbol{\eta}_{2}\right)\right)^{T}
$$

The second coordinate transform relates rotational velocities between body-fixed and earth-fixed coordinates:

$$
\left[\begin{array}{c}
\dot{\phi} \\
\dot{\theta} \\
\dot{\psi}
\end{array}\right]=J_{2}\left(\eta_{2}\right)\left[\begin{array}{l}
p \\
q \\
r
\end{array}\right]
$$

where

$$
J_{2}\left(\eta_{2}\right)=\left[\begin{array}{ccc}
1 & \sin \phi \tan \theta & \cos \phi \tan \theta \\
0 & \cos \phi & -\sin \phi \\
0 & \sin \phi / \cos \theta & \cos \phi / \cos \theta
\end{array}\right]
$$


Note that $J_{2}\left(\eta_{2}\right)$ is not defined for pitch angle $\theta= \pm 90^{\circ}$. This is not a problem, as the vehicle motion does not ordinarily approach this singularity. If we were in a situation where it became necessary to model the vehicle motion through extreme pitch angles, we could resort to an alternate kinematic representation such as quaternions or Rodriguez parameters [17].

\subsection{Vehicle Rigid-Body Dynamics}

The locations of the vehicle centers of gravity and buoyancy are defined in terms of the body-fixed coordinate system as follows:

$$
\boldsymbol{r}_{G}=\left[\begin{array}{l}
x_{g} \\
y_{g} \\
z_{g}
\end{array}\right] \quad \boldsymbol{r}_{B}=\left[\begin{array}{l}
x_{b} \\
y_{b} \\
z_{b}
\end{array}\right]
$$

Given that the origin of the body-fixed coordinate system is located at the center of buoyancy as noted in Section 3.1, the following represent equations of motion for a rigid body in six degrees of freedom, defined in terms of body-fixed coordinates:

$$
\begin{aligned}
m\left[\dot{u}-v r+w q-x_{g}\left(q^{2}+r^{2}\right)+y_{g}(p q-\dot{r})+z_{g}(p r+\dot{q})\right] & =\sum X_{\mathrm{ext}} \\
m\left[\dot{v}-w p+u r-y_{g}\left(r^{2}+p^{2}\right)+z_{g}(q r-\dot{p})+x_{g}(q p+\dot{r})\right] & =\sum Y_{\mathrm{ext}} \\
m\left[\dot{w}-u q+v p-z_{g}\left(p^{2}+q^{2}\right)+x_{g}(r p-\dot{q})+y_{g}(r q+\dot{p})\right] & =\sum Z_{\mathrm{ext}} \\
I_{x x} \dot{p}+\left(I_{z z}-I_{y y}\right) q r-(\dot{r}+p q) I_{x z}+\left(r^{2}-\dot{q}^{2}\right) I_{y z}+(p r-\dot{q}) I_{x y} & \\
+m\left[y_{g}(\dot{w}-u q+v p)-z_{g}(\dot{v}-w p+u r)\right] & =\sum K_{\mathrm{ext}} \\
I_{y y} \dot{q}+\left(I_{x x}-I_{z z}\right) r p-(\dot{p}+q r) I_{x y}+\left(p^{2}-r^{2}\right) I_{x z}+(q p-\dot{r}) I_{y z} & \\
+m\left[z_{g}(\dot{u}-v r+w q)-x_{g}(\dot{w}-u q+v p)\right] & =\sum M_{\mathrm{ext}} \\
I_{z z} \dot{r}+\left(I_{y y}-I_{x x}\right) p q-(\dot{q}+r p) I_{y z}+\left(q^{2}-p^{2}\right) I_{x y}+(r q-\dot{p}) I_{x z} & \\
+m\left[x_{g}(\dot{v}-w p+u r)-y_{g}(\dot{u}-v r+w q)\right] & =\sum N_{\mathrm{ext}}
\end{aligned}
$$

where $m$ is the vehicle mass. The first three equations represent translational motion, the second three rotational motion. Note that these equations neglect the zero-valued center of buoyancy terms.

Given the body-fixed coordinate system centered at the vehicle center of buoyancy, we have the following, diagonal inertia tensor.

$$
I_{o}=\left[\begin{array}{ccc}
I_{x x} & 0 & 0 \\
0 & I_{y y} & 0 \\
0 & 0 & I_{z z}
\end{array}\right]
$$

This is based on the assumption, stated in Section 2.6, that the vehicle products of inertia of inertia are small. 
This simplifies the equations of motion to the following:

$$
\begin{aligned}
m\left[\dot{u}-v r+w q-x_{g}\left(q^{2}+r^{2}\right)+y_{g}(p q-\dot{r})+z_{g}(p r+\dot{q})\right] & =\sum X_{\mathrm{ext}} \\
m\left[\dot{v}-w p+u r-y_{g}\left(r^{2}+p^{2}\right)+z_{g}(q r-\dot{p})+x_{g}(q p+\dot{r})\right] & =\sum Y_{\mathrm{ext}} \\
m\left[\dot{w}-u q+v p-z_{g}\left(p^{2}+q^{2}\right)+x_{g}(r p-\dot{q})+y_{g}(r q+\dot{p})\right] & =\sum Z_{\mathrm{ext}} \\
I_{x x} \dot{p}+\left(I_{z z}-I_{y y}\right) q r+m\left[y_{g}(\dot{w}-u q+v p)-z_{g}(\dot{v}-w p+u r)\right] & =\sum K_{\mathrm{ext}} \\
I_{y y} \dot{q}+\left(I_{x x}-I_{z z}\right) r p+m\left[z_{g}(\dot{u}-v r+w q)-x_{g}(\dot{w}-u q+v p)\right] & =\sum M_{\mathrm{ext}} \\
I_{z z} \dot{r}+\left(I_{y y}-I_{x x}\right) p q+m\left[x_{g}(\dot{v}-w p+u r)-y_{g}(\dot{u}-v r+w q)\right] & =\sum N_{\mathrm{ext}}
\end{aligned}
$$

We can further simply these equations by assuming that $y_{g}$ is small compared to the other terms. Given the layout of the internal components of the REMUS vehicle, unless the vehicle is specially ballasted $y_{g}$ is in fact negligible. This results in the following equations for the vehicle rigid body dynamics:

$$
\begin{aligned}
m\left[\dot{u}-v r+w q-x_{g}\left(q^{2}+r^{2}\right)+z_{g}(p r+\dot{q})\right] & =\sum X_{\mathrm{ext}} \\
m\left[\dot{v}-w p+u r+z_{g}(q r-\dot{p})+x_{g}(q p+\dot{r})\right] & =\sum Y_{\mathrm{ext}} \\
m\left[\dot{w}-u q+v p-z_{g}\left(p^{2}+q^{2}\right)+x_{g}(r p-\dot{q})\right] & =\sum Z_{\mathrm{ext}} \\
I_{x x} \dot{p}+\left(I_{z z}-I_{y y}\right) q r+m\left[-z_{g}(\dot{v}-w p+u r)\right] & =\sum K_{\mathrm{ext}} \\
I_{y y} \dot{q}+\left(I_{x x}-I_{z z}\right) r p+m\left[z_{g}(\dot{u}-v r+w q)-x_{g}(\dot{w}-u q+v p)\right] & =\sum M_{\mathrm{ext}} \\
I_{z z} \dot{r}+\left(I_{y y}-I_{x x}\right) p q+m\left[x_{g}(\dot{v}-w p+u r)\right] & =\sum N_{\mathrm{ext}}
\end{aligned}
$$

\subsection{Vehicle Mechanics}

In the vehicle equations of motion, external forces and moments

$$
\sum F_{\text {ext }}=F_{\text {hydrostatic }}+F_{\text {lift }}+F_{\text {drag }}++F_{\text {control }}
$$

are described in terms of vehicle coefficients. For example, axial drag

$$
F_{\mathrm{d}}=-\left(\frac{1}{2} \rho c_{d} A_{f}\right) u|u|=X_{u|u|} u|u| \quad \Longrightarrow \quad X_{u|u|}=\frac{\partial F_{\mathrm{d}}}{\partial(u|u|)}=-\frac{1}{2} \rho c_{d} A_{f}
$$

These coefficients are based on a combination of theoretical equations and empirically-derived formulae. The actual values of these coefficients are derived Chapter 4. 


\section{Chapter 4}

\section{Coefficient Derivation}

In this chapter, we derive the coefficients defining the forces and moments on the vehicle. The vehicle and fluid parameters necessary for calculating each coefficient are included either in the section describing the coefficient, or are listed in Appendix A.

\subsection{Hydrostatics}

The vehicle experiences hydrostatic forces and moments as a result of the combined effects of the vehicle weight and buoyancy. Let $m$ be the mass of the vehicle. Obviously, the vehicle weight $W=m g$. The vehicle buoyancy is expressed as $B=\rho \nabla g$, where $\rho$ is the density of the surrounding fluid and $\nabla$ the total volume displaced by the vehicle.

It is necessary to express these forces and moments in terms of body-fixed coordinates. This is accomplished using the transformation matrix given in Equation 3.2:

$$
f_{G}\left(\eta_{2}\right)=J_{1}^{-1}\left[\begin{array}{c}
0 \\
0 \\
W
\end{array}\right] \quad f_{B}\left(\eta_{2}\right)=J_{1}^{-1}\left[\begin{array}{c}
0 \\
0 \\
B
\end{array}\right]
$$

The hydrostatic forces and moments on the vehicle can be expressed as:

$$
\begin{aligned}
\boldsymbol{F}_{H S} & =\boldsymbol{f}_{G}-\boldsymbol{f}_{B} \\
\boldsymbol{M}_{H S} & =\boldsymbol{r}_{G} \times \boldsymbol{f}_{G}-\boldsymbol{r}_{B} \times \boldsymbol{f}_{B}
\end{aligned}
$$

These equations can be expanded to yield the nonlinear equations for hydrostatic forces and moments:

$$
\begin{aligned}
X_{H S} & =-(W-B) \sin \theta \\
Y_{H S} & =(W-B) \cos \theta \sin \phi \\
Z_{H S} & =(W-B) \cos \theta \cos \phi \\
K_{H S} & =-\left(y_{g} W-y_{b} B\right) \cos \theta \cos \phi-\left(z_{g} W-z_{b} B\right) \cos \theta \sin \phi \\
M_{H S} & =-\left(z_{g} W-z_{b} B\right) \sin \theta-\left(x_{g} W-x_{b} B\right) \cos \theta \cos \phi \\
N_{H S} & =-\left(x_{g} W-x_{b} B\right) \cos \theta \sin \phi-\left(y_{g} W-y_{b} B\right) \sin \theta
\end{aligned}
$$

Note that the hydrostatic moment is stabilizing in pitch and roll, meaning that the hydrostatic moment opposes deflections in those angular directions. 


\subsection{Hydrodynamic Damping}

It is well known that the damping of an underwater vehicle moving at a high speed in six degrees of freedom is coupled and highly non-linear. In order to simplify modeling the vehicle, we will make the following assumptions:

- We will neglect linear and angular coupled terms. We will assume that terms such as $Y_{r v}$ and $M_{r v}$ are relatively small. Calculating these terms is beyond the scope of this work.

- We will assume the vehicle is top-bottom (xy-plane) and port-starboard (xz-plane) symmetric. We will ignore the vehicle asymmetry caused by the sonar transducer. This allows us to neglect such drag-induced moments as $K_{v|v|}$ and $M_{u|u|}$.

- We will neglect any damping terms greater than second-order. This will allow us to drop such higher-order terms as $Y_{v v v}$.

The principal components of hydrodynamic damping are skin friction due to boundary layers, which are partially laminar and partially turbulent, and damping due to vortex shedding. Nondimensional analysis helps us predict the type of flow around the vehicle. Reynolds number represents the ratio of inertial to viscous forces, and is given by the equation

$$
R e=\frac{U l}{\nu}
$$

where $U$ is the vehicle operating speed, which for REMUS is typically $1.5 \mathrm{~m} / \mathrm{s}$ (3 knots); $l$ the characteristic length, which for REMUS is 1.7 meters; and $\nu$ the fluid kinematic viscosity, which for seawater at $15^{\circ} \mathrm{C}$, Newman [24] gives a value of $1.190 \times 10^{-6} \mathrm{~m}^{2} / \mathrm{s}$.

This yields a Reynolds number of $1.3 \times 10^{6}$, which for a body with a smooth surface falls in the transition zone between laminar and turbulent flow. However, the hull of the REMUS vehicle is broken up by a number of seams, pockets, and bulges, which more than likely trip the flow around the vehicle into the turbulent regime. We can use this information to estimate the drag coefficient of the vehicle.

Note that viscous drag always opposes vehicle motion. In order to result in the proper sign, it is necessary in all equations for drag to consider $v|v|$, as opposed to $v^{2}$.

\subsubsection{Axial Drag}

Vehicle axial drag can be expressed by the following empirical relationship:

$$
X=-\left(\frac{1}{2} \rho c_{\mathrm{d}} A_{f}\right) u|u|
$$

This equation yields the following non-linear axial drag coefficient:

$$
X_{u|u|}=-\frac{1}{2} \rho c_{\mathrm{d}} A_{f}
$$

where $\rho$ is the density of the surrounding fluid, $A_{f}$ the vehicle frontal area, and $c_{\mathrm{d}}$ the axial drag coefficient of the vehicle.

Bottaccini [7, p. 26], Hoerner [15, pg. 3-12] and Triantafyllou [29] offer empirical formulae for calculating the axial drag coefficient. For example, Triantafyllou:

$$
c_{d}=\frac{c_{s s} \pi A_{p}}{A_{f}}\left[1+60\left(\frac{d}{l}\right)^{3}+0.0025\left(\frac{l}{d}\right)\right]
$$


where $c_{s s}$ is Schoenherr's value for flat plate skin friction, $A_{p}=l d$ is the vehicle plan area, and $A_{f}$ is the vehicle frontal area. From Principles of Naval Architecture [20], we get an estimate for $c_{s s}$ of $3.397 \times 10^{-3}$.

These empirical equations yield a value for $c_{\mathrm{d}}$ in the range of 0.11 to 0.13 . Experiments conducted at sea by the Oceanographic Systems Lab measuring the propulsion efficiency of the vehicle resulted in an estimate for $c_{\mathrm{d}}$ of 0.2 .

Full-scale tow tank measurements of the vehicle axial drag-conducted by the author at the University of Rhode Island and described in Chapter 5-yielded an axial drag coefficient of 0.27 . This higher value reflects the drag of the vehicle hull plus the drag of sources neglected in the empirical estimate, such as the vehicle fins and sonar transponder, and the pockets in the vehicle nose section. We will use this higher, experimentally-measured value in the vehicle simulation.

See Table C.1 for the final value of $X_{u|u|}$.

\subsubsection{Crossflow Drag}

Vehicle crossflow drag is considered to be the sum of the hull crossflow drag plus the fin crossflow drag. The method used for calculating the hull drag is analogous to strip theory, the method used to calculate the hull added mass: the total hull drag is approximated as the sum of the drags on the two-dimensional cylindrical vehicle cross-sections.

Slender body theory is a reasonably accurate method for calculating added mass, but for viscous terms it can be off by as much as 100\% [29]. This method does, however, allow us to include all of the terms in the equations of motion. In conducting the vehicle simulation, we will attempt to correct any errors in the crossflow drag terms through comparison with experimental data and observations of the vehicle at sea.

The nonlinear crossflow drag coefficients are expressed as follows:

$$
\begin{gathered}
Y_{v|v|}=Z_{w|w|}=-\frac{1}{2} \rho c_{\mathrm{dc}} \int_{x_{t}}^{x_{b 2}} 2 R(x) \mathrm{d} x-2 \cdot\left(\frac{1}{2} \rho S_{\mathrm{fin}} c_{\mathrm{df}}\right) \\
M_{w|w|}=-N_{v|v|}=\frac{1}{2} \rho c_{\mathrm{dc}} \int_{x_{t}}^{x_{b 2}} 2 x R(x) \mathrm{d} x-2 x_{\mathrm{fin}} \cdot\left(\frac{1}{2} \rho S_{\mathrm{fin}} c_{\mathrm{df}}\right) \\
Y_{r|r|}=-Z_{q|q|}=-\frac{1}{2} \rho c_{\mathrm{dc}} \int_{x_{t}}^{x_{b 2}} 2 x|x| R(x) \mathrm{d} x-2 x_{\mathrm{fin}}\left|x_{\mathrm{fin}}\right| \cdot\left(\frac{1}{2} \rho S_{\mathrm{fin}} c_{\mathrm{df}}\right) \\
M_{q|q|}=N_{r|r|}=-\frac{1}{2} \rho c_{\mathrm{dc}} \int_{x_{t}}^{x_{b 2}} 2 x^{3} R(x) \mathrm{d} x-2 x_{\mathrm{fin}}^{3} \cdot\left(\frac{1}{2} \rho S_{\mathrm{fin}} c_{\mathrm{df}}\right)
\end{gathered}
$$

where $\rho$ is the seawater density, $c_{\mathrm{dc}}$ the drag coefficient of a cylinder, $R(x)$ the hull radius as a function of axial position as given by Equations 2.1 and $2.2, S_{\text {fin }}$ the control fin planform area, and $c_{\mathrm{df}}$ the crossflow drag coefficient of the control fins. See Table A.2 for the limits of integration.

Hoerner [15] estimates the crossflow drag coefficient of a cylinder $c_{\mathrm{dc}}$ to be 1.1. The crossflow drag coefficient $c_{\mathrm{df}}$ is derived using the formula developed by Whicker and Fehlner [32]:

$$
c_{\mathrm{df}}=0.1+0.7 t
$$

where $t$ is the fin taper ratio, or the ratio of the widths of the top and bottom of the fin along the vehicle long axis. From this formula, we get an estimate for $c_{\mathrm{df}}$ of 0.56 .

See Table C.2 for the final coefficient values. 


\subsubsection{Rolling Drag}

We will approximate the rolling resistance of the vehicle by assuming that the principle component comes from the crossflow drag of the fins.

$$
F=\left(Y_{v v f} r_{\text {mean }}\right) r_{\text {mean }}^{2} p|p|
$$

where $Y_{v v f}$ is the fin component of the vehicle crossflow drag coefficient, and $r_{\text {mean }}$ is the mean fin height above the vehicle centerline. This yields the following equation for the vehicle rolling drag coefficient:

$$
K_{p|p|}=Y_{v v f} r_{\text {mean }}^{3}
$$

This is at best a rough approximation for the actual value. It would be better to use experimental data.

See Table C.3 for the coefficient value based on this rough approximation.

\subsection{Added Mass}

Added mass is a measure of the mass of the moving water when the vehicle accelerates. Ideal fluid forces and moments can be expressed by the equations:

$$
\begin{aligned}
& F_{j}=-\dot{u}_{\mathrm{i}} m_{\mathrm{ji}}-\varepsilon_{\mathrm{jkl}} u_{\mathrm{i}} \Omega_{\mathrm{k}} m_{\mathrm{li}} \\
& M_{j}=-\dot{u}_{\mathrm{i}} m_{\mathrm{j}+3, \mathrm{i}}-\varepsilon_{\mathrm{jk} \mathrm{l}} u_{\mathrm{i}} \Omega_{\mathrm{k}} m_{\mathrm{l}+3, \mathrm{i}}-\varepsilon_{\mathrm{jkl}} u_{\mathrm{k}} u_{\mathrm{i}} m_{\mathrm{li}} \\
& \quad \text { where } \quad i=1,2,3,4,5,6 \\
& \quad \text { and } j k l=1,2,3
\end{aligned}
$$

and where the alternating tensor $\varepsilon_{\mathrm{jkl}}$ is equal to +1 if the indices are in cyclic order $(123,231$, $312),-1$ if the indices are acyclic $(132,213,321)$, and zero if any pair of the indices are equal. See Newman [24] or Fossen [10] for the expansion of these equations.

Due to body top-bottom and port-starboard symmetry, the vehicle added mass matrix reduces to:

$$
\left[\begin{array}{cccccc}
m_{11} & 0 & 0 & 0 & 0 & 0 \\
0 & m_{22} & 0 & 0 & 0 & m_{26} \\
0 & 0 & m_{33} & 0 & m_{35} & 0 \\
0 & 0 & 0 & m_{44} & 0 & 0 \\
0 & 0 & m_{53} & 0 & m_{55} & 0 \\
0 & m_{62} & 0 & 0 & 0 & m_{66}
\end{array}\right]
$$

which is equivalent to:

$$
\left[\begin{array}{cccccc}
X_{\dot{u}} & 0 & 0 & 0 & 0 & 0 \\
0 & Y_{\dot{v}} & 0 & 0 & 0 & N_{\dot{v}} \\
0 & 0 & Z_{\dot{w}} & 0 & M_{\dot{w}} & 0 \\
0 & 0 & 0 & K_{\dot{p}} & 0 & 0 \\
0 & 0 & Z_{\dot{q}} & 0 & M_{\dot{q}} & 0 \\
0 & Y_{\dot{r}} & 0 & 0 & 0 & N_{\dot{r}}
\end{array}\right]
$$

Substituting these remaining terms into the expanded equations for fluid forces and moments 
from Equation 4.12 yields the following equations:

$$
\begin{aligned}
X_{\mathrm{A}} & =X_{\dot{u}} \dot{u}+Z_{\dot{w}} w q+Z_{\dot{q}} q^{2}-Y_{\dot{v}} v r-Y_{\dot{r}} \dot{r}^{2} \\
Y_{\mathrm{A}} & =Y_{\dot{v}} \dot{v}+Y_{\dot{r}} \dot{r}+X_{\dot{u}} u r-Z_{\dot{w}} w p-Z_{\dot{q}} p q \\
Z_{\mathrm{A}} & =Z_{\dot{w}} \dot{w}+Z_{\dot{q}} \dot{q}-X_{\dot{u}} u q+Y_{\dot{v}} v p+Y_{\dot{r}} r p \\
K_{\mathrm{A}} & =K_{\dot{p}} \dot{p} \\
M_{\mathrm{A}} & =M_{\dot{w}} \dot{w}+M_{\dot{q}} \dot{q}-\left(Z_{\dot{w}}-X_{\dot{u}}\right) u w-Y_{\dot{r}} v p+\left(K_{\dot{p}}-N_{\dot{r}}\right) r p-Z_{\dot{q}} u q \\
N_{\mathrm{A}} & =N_{\dot{v}} \dot{v}+N_{\dot{r}} \dot{r}-\left(X_{\dot{u}}-Y_{\dot{v}}\right) u v+Z_{\dot{q}} w p-\left(K_{\dot{p}}-M_{\dot{q}}\right) p q+Y_{\dot{r}} u r
\end{aligned}
$$

\subsubsection{Axial Added Mass}

To estimate axial added mass, we approximate the vehicle hull shape by an ellipsoid for which the major axis is half the vehicle length $l$, and the minor axis half the vehicle diameter $d$. See Figure 2-4 for a comparison of the two shapes. Blevins $[6, p .407]$ gives the following empirical formula for the axial added mass of an ellipsoid:

$$
X_{\dot{u}}=-m_{11}=-\frac{4 \alpha \rho \pi}{3}\left(\frac{l}{2}\right)\left(\frac{d}{2}\right)^{2}
$$

or

$$
X_{\dot{u}}=-m_{11}=-\frac{4 \beta \rho \pi}{3}\left(\frac{d}{2}\right)^{3}
$$

where $\rho$ is the density of the surrounding fluid, and $\alpha$ and $\beta$ are empirical parameters measured by Blevins and determined by the ratio of the vehicle length to diameter as shown in Table 4.1.

Table 4.1: Axial Added Mass Parameters $\alpha$ and $\beta$

\begin{tabular}{rlc}
\hline \hline$l / d$ & \multicolumn{1}{c}{$\alpha$} & \multicolumn{1}{c}{$\beta$} \\
\hline 0.01 & - & 0.6348 \\
0.1 & 6.148 & 0.6148 \\
0.2 & 3.008 & 0.6016 \\
0.4 & 1.428 & 0.5712 \\
0.6 & 0.9078 & 0.5447 \\
0.8 & 0.6514 & 0.5211 \\
1.0 & 0.5000 & 0.5000 \\
1.5 & 0.3038 & 0.4557 \\
2.0 & 0.2100 & 0.4200 \\
2.5 & 0.1563 & 0.3908 \\
3.0 & 0.1220 & 0.3660 \\
5.0 & 0.05912 & 0.2956 \\
7.0 & 0.03585 & 0.2510 \\
10.0 & 0.02071 & 0.2071 \\
\hline \hline
\end{tabular}

See Table C.5 for the final coefficient values.

\subsubsection{Crossflow Added Mass}

Vehicle added mass is calculated using strip theory on both cylindrical and cruciform hull cross sections. From Newman [24], the added mass per unit length of a single cylindrical slice is given as:

$$
m_{a}(x)=\pi \rho R(x)^{2}
$$


where $\rho$ is the density of the surrounding fluid, and $R(x)$ the hull radius as a function of axial position as given by Equations 2.1 and 2.2. The added mass of a circle with fins is given in Blevins [6] as:

$$
m_{a f}(x)=\pi \rho\left(a_{\text {fin }}^{2}-R(x)^{2}+\frac{R(x)^{4}}{a_{\text {fin }}^{2}}\right)
$$

where $a_{\text {fin }}$, as defined in Table 2.2, is the maximum height above the centerline of the vehicle fins.

Integrating Equations 4.18 and 4.19 over the length of the vehicle, we arrive at the following equations for crosslow added mass:

$$
\begin{aligned}
Y_{\dot{v}} & =-m_{22}=-\int_{x_{\mathrm{t}}}^{x_{\mathrm{f}}} m_{a}(x) \mathrm{d} x-\int_{x_{\mathrm{f}}}^{x_{\mathrm{f} 2}} m_{a f}(x) \mathrm{d} x-\int_{x_{\mathrm{f} 2}}^{x_{\mathrm{b} 2}} m_{a}(x) \mathrm{d} x \\
Z_{\dot{w}} & =-m_{33}=-m_{22}=Y_{\dot{v}} \\
M_{\dot{w}} & =-m_{53}=\int_{x_{\mathrm{t}}}^{x_{\mathrm{f}}} x m_{a}(x) \mathrm{d} x-\int_{x_{\mathrm{f}}}^{x_{\mathrm{f} 2}} x m_{a f}(x) \mathrm{d} x-\int_{x_{\mathrm{f} 2}}^{x_{\mathrm{b} 2}} x m_{a}(x) \mathrm{d} x \\
N_{\dot{v}} & =-m_{62}=m_{53}=-M_{\dot{w}} \\
Y_{\dot{r}} & =-m_{26}=-m_{62}=N_{\dot{v}} \\
Z_{\dot{q}} & =-m_{35}=-m_{53}=M_{\dot{w}} \\
M_{\dot{q}} & =-m_{55}=-\int_{x_{\mathrm{tail}}}^{x_{\mathrm{fin}}} x^{2} m_{a}(x) \mathrm{d} x-\int_{x_{\mathrm{fin}}}^{x_{\mathrm{fin} 2}} x^{2} m_{a f}(x) \mathrm{d} x-\int_{x_{\mathrm{fin} 2}}^{x_{\mathrm{bow} 2}} x^{2} m_{a}(x) \mathrm{d} x \\
N_{\dot{r}} & =-m_{66}=-m_{55}=M_{\dot{q}}
\end{aligned}
$$

See Table A.2 for the limits of integration.

See Table C.5 for the final coefficient values.

\subsubsection{Rolling Added Mass}

To estimate rolling added mass, we will assume that the relatively smooth sections of the vehicle hull do not generate any added mass in roll. We will also neglect the added mass generated by the sonar transponder and any other small protuberances. Given those assumptions, we need only consider the hull section containing the vehicle control fins.

Blevins [6] offers the following empirical formula for the added mass of a rolling circle with fins:

$$
K_{\dot{p}}=-\int_{x_{\mathrm{fin}}}^{x_{\mathrm{fin} 2}} \frac{2}{\pi} \rho a^{4} \mathrm{~d} x
$$

where $a$ is the fin height above the vehicle centerline, in this case averaged to be $0.1172 \mathrm{~m}$. See Table A.2 for the limits of integration.

See Table C.5 for the final coefficient value.

\subsubsection{Added Mass Cross-terms}

The remaining cross-terms result from added mass coupling, and are listed below:

$$
\begin{aligned}
& \begin{array}{llll}
X_{w q}=Z_{\dot{w}} & X_{q q}=Z_{\dot{q}} \quad X_{v r}=-Y_{\dot{v}} \quad X_{r r}=-Y_{\dot{r}}
\end{array} \\
& Y_{u r}=X_{\dot{u}} \quad Y_{w p}=-Z_{\dot{w}} \quad Y_{p q}=-Z_{\dot{q}} \\
& Z_{u q}=-X_{\dot{u}} \quad Z_{v p}=Y_{\dot{v}} \quad Z_{r p}=Y_{\dot{r}} \\
& M_{u w a}=-\left(Z_{\dot{w}}-X_{\dot{u}}\right) \quad M_{v p}=-Y_{\dot{r}} \quad M_{r p}=\left(K_{\dot{p}}-N_{\dot{r}}\right) \quad M_{u q}=-Z_{\dot{q}} \\
& N_{u v a}=-\left(X_{\dot{u}}-Y_{\dot{v}}\right) \quad N_{w p}=Z_{\dot{q}} \quad N_{p q}=-\left(K_{\dot{p}}-M_{\dot{q}}\right) \quad N_{u r}=Y_{\dot{r}}
\end{aligned}
$$


The added mass cross-terms $M_{u w a}$ and $N_{u v a}$ are known as the Munk Moment, and relates to the pure moment experienced by a body at an angle of attack in ideal, inviscid flow.

See Tables C.6, C.7 and C.8 for the final coefficient values.

\subsection{Body Lift}

Vehicle body lift results from the vehicle moving through the water at an angle of attack, causing How separation and a subsequent drop in pressure along the aft, upper section of the vehicle hull. This pressure drop is modeled as a point force applied at the center of pressure. As this center of pressure does not line up with the origin of the vehicle-fixed coordinate system, this force also leads to a pitching moment about the origin.

In determining the best method for calculating body lift, the author compared three empirical methods based on torpedo data $[7,9,16]$, and one theoretical method [23]. Unfortunately, the estimates for body lift from the four methods ranged over an order of magnitude. Given the lack of agreement between the empirical methods, it would be preferable to base the body lift estimates on actual REMUS data, from perhaps tow tank tests or measurements of the vehicle mounted on a rotating arm.

Until that happens, the author decided to use Hoerner's estimates [16], which appeared the most reliable.

\subsubsection{Body Lift Force}

To calculate body lift, we will use the empirical formula developed by Hoerner [16], which states:

$$
L_{\text {body }}=-\frac{1}{2} \rho d^{2} c_{y d} u^{2}
$$

where $\rho$ is the density of the surrounding fluid, $A_{p}$ the projected area of the vehicle hull, $u$ the vehicle forward velocity, and $c_{y d}$ the body lift coefficient, which by Hoerner's notation is expressed as:

$$
c_{y d}=c_{y d}(\beta)=\frac{\mathrm{d} c_{y d}}{\mathrm{~d} \beta} \beta
$$

where $\beta$ is the vehicle angle of attack in radians and is given by the relationship :

$$
\tan \beta=\frac{w}{u} \quad \Longrightarrow \quad \beta \approx \frac{w}{u}
$$

Hoerner gives the following relationship for lift slope:

$$
\frac{\mathrm{d} c_{y d}^{\circ}}{\mathrm{d} \beta^{\circ}}=c_{y d \beta}^{\circ}=\left(\frac{l}{d}\right) c_{y \beta}^{\circ}
$$

where $l$ is the vehicle length and $d$ the maximum diameter. Hoerner [16, pg. 13-3] states that

$$
\text { for } \quad 6.7 \leq \frac{l}{d} \leq 10, \quad c_{y \beta}^{\circ}=0.003
$$

Note that in Equation 4.30 it is necessary to convert the Hoerner lift slope coefficients $c_{y d \beta}^{\circ}$ and $c_{y \beta}^{\circ}$ from degree to radians. This results in the Hoerner lift slope coefficient $c_{y d \beta}$, defined in terms of radians as follows:

$$
c_{y d \beta}=c_{y d \beta}^{\circ}\left(\frac{180}{\pi}\right)
$$


Substituting into Equation 4.27 the relationships given above, we are left with the following equation for vehicle body lift:

$$
L_{\mathrm{body}}=-\frac{1}{2} \rho d^{2} c_{y d \beta} u w
$$

which results in the following body lift coefficients:

$$
Y_{u v l}=Z_{u w l}=-\frac{1}{2} \rho d^{2} c_{y d \beta}
$$

See Table C.4 for the final coefficient values.

\subsubsection{Body Lift Moment}

Hoerner estimates that for a body of revolution at an angle of attack, the viscous force is centered at a point between 0.6 and 0.7 of the total body length from the nose. His experimental findings suggest that the flow goes smoothly around the forward end of the hull, and that the lateral force only develops on the leeward side of the rear half of the hull.

Following these findings, we will assume that, in body-fixed coordinates:

$$
x_{c p}=-0.65 l-x_{z e r o}
$$

This results in the following equation for body lift moment:

$$
M_{u w l}=-N_{u v l}=-\frac{1}{2} \rho d^{2} c_{y d \beta} x_{c p}
$$

See Table C.4 for the final coefficient values.

\subsection{Fin Lift}

The attitude of the REMUS vehicle is controlled by two horizontal fins, or stern planes, and two vertical fins, or rudders. The pairs of fins move together; in other words the stern planes do not move independently of each other, nor do the rudder planes.

For the vehicle control fins, the empirical formula for fin lift is given as:

$$
\begin{aligned}
L_{\mathrm{fin}} & =\frac{1}{2} \rho c_{L} S_{\mathrm{fin}} \delta_{e} v_{\mathrm{e}}^{2} \\
M_{\mathrm{fin}} & =x_{\mathrm{fin}} L_{\mathrm{fin}}
\end{aligned}
$$

where $c_{L}$ is the fin lift coefficient, $S_{\text {fin }}$ the fin planform area, $\delta_{e}$ the effective fin angle in radians, $v_{\mathbf{e}}$ the effective fin velocity, and $x_{\text {fin }}$ the axial position of the fin post in body-referenced coordinates.

Fin lift coefficient $c_{L}$ is a function of the effective fin angle of attack $\alpha$. Hoerner [16, pg. 3-2] gives the following empirical formula for fin lift as a function of $\alpha$ in radians:

$$
c_{L \alpha}=\frac{\mathrm{d} c_{l}}{\mathrm{~d} \alpha}=\left[\frac{1}{2 \bar{\alpha} \pi}+\frac{1}{\pi\left(\mathrm{AR}_{e}\right)}\right]^{-1}
$$

where the factor $\bar{\alpha}$ was found by Hoerner to be of the order 0.9 , and $\left(\mathrm{AR}_{e}\right)$ is the effective fin aspect ratio, which is given by the formula:

$$
\mathrm{AR}_{e}=2(\mathrm{AR})=2\left(\frac{b_{\mathrm{fin}}^{2}}{S_{\mathrm{fin}}}\right)
$$


As the fin is located at some offset from the origin of the vehicle coordinate system, it experiences the following effective velocities:

$$
\begin{gathered}
u_{\mathrm{fin}}=u+z_{\mathrm{fin}} q-y_{\mathrm{fin}} r \\
v_{\mathrm{fin}}=v+x_{\mathrm{fin}} r-z_{\mathrm{fin}} p \\
w_{\mathrm{fin}}=w+y_{\mathrm{fin}} p-x_{\mathrm{fin}} q
\end{gathered}
$$

where $x_{\text {fin }}, y_{\text {fin }}$, and $z_{\text {fin }}$ are the body-referenced coordinates of the fin posts. For the REMUS vehicle, we will drop the terms involving $y_{\mathrm{fin}}$ and $z_{\mathrm{fin}}$ as they are small compared to the vehicle translational velocities.

The effective fin angles $\delta_{s e}$ and $\delta_{r e}$ can be expressed as

$$
\begin{aligned}
& \delta_{r e}=\delta_{r}-\beta_{r e} \\
& \delta_{s e}=\delta_{s}+\beta_{s e}
\end{aligned}
$$

where $\delta_{s}$ and $\delta_{r}$ are the fin angles referenced to the vehicle hull, $\beta_{s e}$ and $\beta_{r e}$ the effective angles of attack of the fin zero plane, as shown in Figures 4-1 and 4-2. Assuming small angles, these effective angles can be expressed as:

$$
\begin{aligned}
& \beta_{r e}=\frac{v_{\mathrm{fin}}}{u_{\mathrm{fin}}} \approx \frac{1}{u}\left(v+x_{\mathrm{fin}} r\right) \\
& \beta_{\text {se }}=\frac{w_{\mathrm{fin}}}{u_{\mathrm{fin}}} \approx \frac{1}{u}\left(w-x_{\mathrm{fin}} q\right)
\end{aligned}
$$

based on Equation 4.40

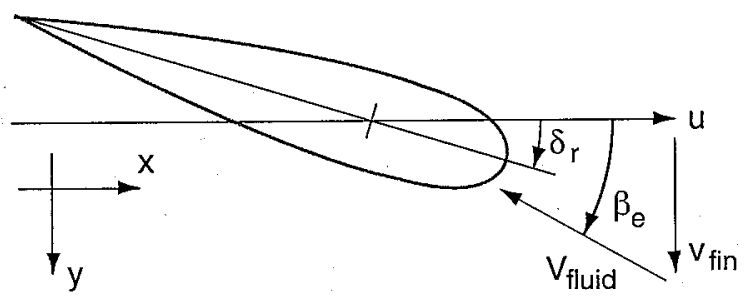

Figure 4-1: Effective Rudder Angle of Attack

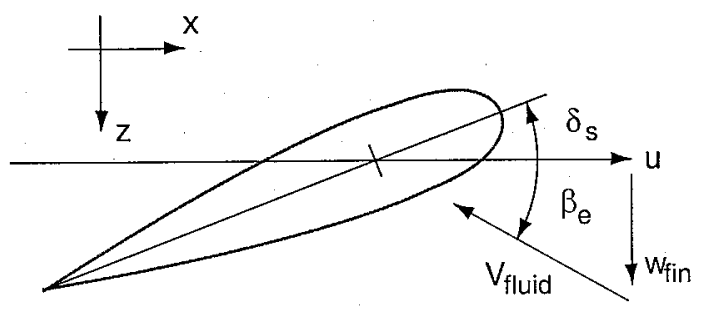

Figure 4-2: Effective Stern Plane Angle of Attack

Substituting the results of Equations 4.40, 4.41 and 4.42 into Equation 4.44 results in the following 
equations for fin lift and moment:

$$
\begin{aligned}
Y_{r} & =\frac{1}{2} \rho c_{L \alpha} S_{\text {fin }}\left[u^{2} \delta_{r}-u v-x_{\text {fin }}(u r)\right] \\
Z_{s} & =-\frac{1}{2} \rho c_{L \alpha} S_{\text {fin }}\left[u^{2} \delta_{s}+u w-x_{\text {fin }}(u q)\right] \\
M_{s} & =\frac{1}{2} \rho c_{L \alpha} S_{\text {fin }} x_{\text {fin }}\left[u^{2} \delta_{s}+u w-x_{\text {fin }}(u q)\right] \\
N_{r} & =\frac{1}{2} \rho c_{L \alpha} S_{\text {fin }} x_{\text {fin }}\left[u^{2} \delta_{r}-u v-x_{\text {fin }}(u r)\right]
\end{aligned}
$$

Finally, we can separate the equation into the following sets of fin lift coefficients:

$$
\begin{aligned}
Y_{u u \delta_{r}} & =-Y_{u v f}=\rho c_{L \alpha} S_{\text {fin }} \\
Z_{u u \delta_{s}} & =Z_{u w f}=-\rho c_{L \alpha} S_{\text {fin }} \\
Y_{u r f} & =-Z_{u q f}=-\rho c_{L \alpha} S_{\text {fin }} x_{\text {fin }}
\end{aligned}
$$

and fin moment coefficients:

$$
\begin{aligned}
M_{u u \delta_{s}} & =M_{u w f}=\rho c_{L \alpha} S_{\mathrm{fin}} x_{\mathrm{fin}} \\
N_{u u \delta_{r}} & =-N_{u v f}=\rho c_{L \alpha} S_{\mathrm{fin}} x_{\mathrm{fin}} \\
M_{u q f} & =N_{u r f}=-\rho c_{L \alpha} S_{\mathrm{fin}} x_{\mathrm{fin}}^{2}
\end{aligned}
$$

See Table C.10 for the final coefficient values.

\subsection{Propulsion Model}

We will use a very simple model for the REMUS propulsion system, which treats the propeller as a source of constant thrust and torque. The values for these coefficients are derived from both vehicle design-stage propeller bench tests conducted by Ben Allen at the Oceanographic Systems Laboratory, and from experiments at sea conducted by the author.

This simple model is acceptable for small amplitude perturbations about the vehicle steady state. If examination of the simulator output indicates that a more sophisticated model is necessary, we can try replacing this with a propeller model, such as Yoerger and Slotine's [35], or with experimentallyderived values.

\subsubsection{Propeller Thrust}

In tests at sea, the REMUS vehicle has been observed to maintain a forward speed of $1.51 \mathrm{~m} / \mathrm{s}$ (3 knots) at a propeller speed of 1500 RPM. We will assume that at this steady velocity, the propeller thrust matches the vehicle axial drag.

$$
\begin{aligned}
X_{\text {prop }} & =-X_{u|u|} u|u| \\
& =-2.28 X_{u|u|}
\end{aligned}
$$

For the purpose of simulation, we will assume that the vehicle makes only small deviations from this forward speed. See Table C.9 for the final coefficient value.

\subsubsection{Propeller Torque}

In sea trials, the REMUS vehicle running at 1500 RPM in steady conditions and zero pitch angle was observed to maintain an average roll offset $\phi$ of -5.3 degrees $\left(-9.3 \times 10^{-2}\right.$ radians). We will 
assume that under these conditions, the propeller torque matches the hydrostatic roll moment.

$$
\begin{aligned}
K_{\text {prop }} & =-K_{H S}=\left(y_{g} W-y_{b} B\right) \cos \theta \cos \phi+\left(z_{g} W-z_{b} B\right) \cos \theta \sin \phi \\
& =0.995\left(y_{g} W-y_{b} B\right)-0.093\left(z_{g} W-z_{b} B\right)
\end{aligned}
$$

See Table C.9 for the final coefficient value.

\subsection{Combined Terms}

Combining like terms from Equations 4.22, 4.34, 4.36, 4.44 and 4.45, we get the following:

$$
\begin{aligned}
Y_{u v} & =Y_{u v l}+Y_{u v f} \\
Y_{u r} & =Y_{u r a}+Y_{u r f} \\
Z_{u w} & =Z_{u w l}+Z_{u w f} \\
Z_{u q} & =Z_{u q a}+Z_{u q f} \\
M_{u w} & =M_{u w a}+M_{u w l}+M_{u w f} \\
M_{u q} & =M_{u q a}+M_{u q f} \\
N_{u v} & =N_{u v a}+N_{u v l}+N_{u v f} \\
N_{u r} & =N_{u r a}+N_{u r f}
\end{aligned}
$$

\subsection{Total Vehicle Forces and Moments}

Combining the coefficient equations for the vehicle

- Hydrostatics: Equation 4.3

- Hydrodynamic Damping: Equations 4.6, 4.8 and 4.11

- Added Mass: Equations 4.16, 4.20, 4.21 and 4.22

- Body Lift and Moment: Equations 4.34 and 4.36

- Fin Lift and Moment: Equations 4.44 and 4.45

- Propeller Thrust and Torque: Equations 4.46 and 4.47 
the sum of the depth-plane forces and moments on the vehicle can be expressed as:

$$
\begin{aligned}
\sum X_{\mathrm{ext}}= & X_{H S}+X_{u|u|} u|u|+X_{\dot{u}} \dot{u}+X_{w q} w q+X_{q q} q q+X_{v r} v r+X_{r r} r r \\
& +X_{\mathrm{prop}} \\
\sum Y_{\mathrm{ext}}= & Y_{H S}+Y_{v|v|} v|v|+Y_{r|r|} r|r|+Y_{\dot{v}} \dot{v}+Y_{\dot{r}} \dot{r} \\
& +Y_{u r} u r+Y_{w p} w p+Y_{p q} p q+Y_{u v} u v+Y_{u u \delta_{r}} u^{2} \delta_{r} \\
\sum Z_{\mathrm{ext}}= & Z_{H S}+Z_{w|w|} w|w|+Z_{q|q|} q|q|+Z_{\dot{w}} \dot{w}+Z_{\dot{q} \dot{q}} \\
& +Z_{u q} u q+Z_{v p} v p+Z_{r p} r p+Z_{u w} u w+Z_{u u \delta_{s}} u^{2} \delta_{s} \\
\sum K_{\mathrm{ext}}= & K_{H S}+K_{p|p|} p|p|+K_{\dot{p} \dot{p}}+K_{\mathrm{prop}} \\
\sum M_{\mathrm{ext}}= & M_{H S}+M_{w|w|} w|w|+M_{q|q|} q|q|+M_{\dot{w}} \dot{w}+M_{\dot{q}} \dot{q} \\
& +M_{u q} u q+M_{v p} v p+M_{r p} r p+M_{u w} u w+M_{u u \delta_{s}} u^{2} \delta_{s} \\
\sum N_{\mathrm{ext}}= & N_{H S}+N_{v|v|} v|v|+N_{r|r|} r|r|+N_{\dot{v}} \dot{v}+N_{\dot{r}} \dot{r} \\
& +N_{u r} u r+N_{w p} w p+N_{p q} p q+N_{u v} u v+N_{u u \delta_{r}} u^{2} \delta_{r}
\end{aligned}
$$

\begin{tabular}{|c|c|c|c|}
\hline Parameter & Value & Units & Description \\
\hline$X_{u u}$ & $-1.62 e+000$ & $\mathrm{~kg} / \mathrm{m}$ & Cross-flow Drag \\
\hline$X_{\dot{u}}$ & $-9.30 e-001$ & $\mathrm{~kg}$ & Added Mass \\
\hline$X_{w q}$ & $-3.55 e+001$ & $\mathrm{~kg} / \mathrm{rad}$ & Added Mass Cross-term \\
\hline$X_{q q}$ & $-1.93 e+000$ & $\mathrm{~kg} \cdot \mathrm{m} / \mathrm{rad}$ & Added Mass Cross-term \\
\hline$X_{v r}$ & $+3.55 e+001$ & $\mathrm{~kg} / \mathrm{rad}$ & Added Mass Cross-term \\
\hline$X_{r r}$ & $-1.93 e+000$ & $\mathrm{~kg} \cdot \mathrm{m} / \mathrm{rad}$ & Added Mass Cross-term \\
\hline$X_{\text {prop }}$ & $+3.86 \mathrm{e}+000$ & $\mathrm{~N}$ & Propeller Thrust \\
\hline$Y_{v v}$ & $-1.31 e+002$ & $\mathrm{~kg} / \mathrm{m}$ & Cross-flow Drag \\
\hline$Y_{r r}$ & $+6.32 \mathrm{e}-001$ & $\mathrm{~kg} \cdot \mathrm{m} / \mathrm{rad}^{2}$ & Cross-flow Drag \\
\hline$Y_{u v}$ & $-2.86 e+001$ & $\mathrm{~kg} / \mathrm{m}$ & Body Lift Force and Fin Lift \\
\hline$Y_{\dot{v}}$ & $-3.55 e+001$ & $\mathrm{~kg}$ & Added Mass \\
\hline$Y_{\dot{r}}$ & $+1.93 e+000$ & $\mathrm{~kg} \cdot \mathrm{m} / \mathrm{rad}$ & Added Mass \\
\hline$Y_{\text {ur }}$ & $+5.22 e+000$ & $\mathrm{~kg} / \mathrm{rad}$ & Added Mass Cross Term and Fin Lift \\
\hline$Y_{w p}$ & $+3.55 e+001$ & $\mathrm{~kg} / \mathrm{rad}$ & Added Mass Cross-term \\
\hline$Y_{p q}$ & $+1.93 e+000$ & $\mathrm{~kg} \cdot \mathrm{m} / \mathrm{rad}$ & Added Mass Cross-term \\
\hline$Y_{u u d r}$ & $+9.64 e+000$ & $\mathrm{~kg} /(\mathrm{m} \cdot \mathrm{rad})$ & Fin Lift Force \\
\hline$Z_{w w}$ & $-1.31 e+002$ & $\mathrm{~kg} / \mathrm{m}$ & Cross-flow Drag \\
\hline$Z_{q q}$ & $-6.32 e-001$ & $\mathrm{~kg} \cdot \mathrm{m} / \mathrm{rad}^{2}$ & Cross-flow Drag \\
\hline$Z_{u w}$ & $-2.86 e+001$ & $\mathrm{~kg} / \mathrm{m}$ & Body Lift Force and Fin Lift \\
\hline$Z_{i \mathfrak{w}}$ & $-3.55 e+001$ & $\mathrm{~kg}$ & Added Mass \\
\hline$Z_{\dot{q}}$ & $-1.93 e+000$ & $\mathrm{~kg} \cdot \mathrm{m} / \mathrm{rad}$ & Added Mass \\
\hline$Z_{u q}$ & $-5.22 e+000$ & $\mathrm{~kg} / \mathrm{rad}$ & Added Mass Cross-term and Fin Lift \\
\hline$Z_{v p}$ & $-3.55 e+001$ & $\mathrm{~kg} / \mathrm{rad}$ & Added Mass Cross-term \\
\hline$Z_{r p}$ & $+1.93 e+000$ & $\mathrm{~kg} / \mathrm{rad}$ & Added Mass Cross-term \\
\hline$Z_{\text {uuds }}$ & $-9.64 e+000$ & $\mathrm{~kg} /(\mathrm{m} \cdot \mathrm{rad})$ & Fin Lift Force \\
\hline
\end{tabular}

See Tables 4.2 and 4.3 for a list of the non-zero vehicle coefficients.

Table 4.2: STD REMUS Non-Linear Maneuvering Coefficients: Forces 
Table 4.3: STD REMUS Non-Linear Maneuvering Coefficients: Moments

\begin{tabular}{cccl}
\hline \hline Parameter & Value & Units & Description \\
\hline$K_{p p}$ & $-1.30 \mathrm{e}-003$ & $\mathrm{~kg} \cdot \mathrm{m}^{2} / \mathrm{rad}^{2}$ & Rolling Resistance \\
$K_{\dot{p}}$ & $-1.41 \mathrm{e}-002$ & $\mathrm{~kg} \cdot \mathrm{m}^{2} / \mathrm{rad}$ & Added Mass \\
$K_{p r o p}$ & $-5.43 \mathrm{e}-001$ & $\mathrm{~N} \cdot \mathrm{m}$ & Propeller Torque \\
$M_{w w}$ & $+3.18 \mathrm{e}+000$ & $\mathrm{~kg}$ & Cross-flow Drag \\
$M_{q q}$ & $-9.40 \mathrm{e}+000$ & $\mathrm{~kg} \cdot \mathrm{m}^{2} / \mathrm{rad}^{2}$ & Cross-flow Drag \\
$M_{u w}$ & $+2.40 \mathrm{e}+001$ & $\mathrm{~kg}$ & Body and Fin Lift and Munk Moment \\
$M_{\dot{w}}$ & $-1.93 \mathrm{e}+000$ & $\mathrm{~kg} \cdot \mathrm{m}$ & Added Mass \\
$M_{\dot{q}}$ & $-4.88 \mathrm{e}+000$ & $\mathrm{~kg} \cdot \mathrm{m}^{2} / \mathrm{rad}$ & Added Mass \\
$M_{u q}$ & $-2.00 \mathrm{e}+000$ & $\mathrm{~kg} \cdot \mathrm{m} / \mathrm{rad}$ & Added Mass Cross Term and Fin Lift \\
$M_{v p}$ & $-1.93 e+000$ & $\mathrm{~kg} \cdot \mathrm{m} / \mathrm{rad}$ & Added Mass Cross Term \\
$M_{r p}$ & $+4.86 e+000$ & $\mathrm{~kg} \cdot \mathrm{m}{ }^{2} / \mathrm{rad}$ & Added Mass Cross-term \\
$M_{u u d s}$ & $-6.15 e+000$ & $\mathrm{~kg} / \mathrm{rad}$ & Fin Lift Moment \\
$N_{v v}$ & $-3.18 \mathrm{e}+000$ & $\mathrm{~kg}$ & Cross-flow Drag \\
$N_{r r}$ & $-9.40 \mathrm{e}+000$ & $\mathrm{~kg} \cdot \mathrm{m}^{2} / \mathrm{rad}$ & Cross-flow Drag \\
$N_{u v}$ & $-2.40 e+001$ & $\mathrm{~kg}$ & Body and Fin Lift and Munk Morment \\
$N_{\dot{v}}$ & $+1.93 e+000$ & $\mathrm{~kg} \cdot \mathrm{m}$ & Added Mass \\
$N_{\dot{r}}$ & $-4.88 \mathrm{e}+000$ & $\mathrm{~kg} \cdot \mathrm{m}^{2} / \mathrm{rad}$ & Added Mass \\
$N_{u r}$ & $-2.00 e+000$ & $\mathrm{~kg} \cdot \mathrm{m} / \mathrm{rad}^{2}$ & Added Mass Cross Term and Fin Lift \\
$N_{w p}$ & $-1.93 e+000$ & $\mathrm{~kg} \cdot \mathrm{m} / \mathrm{rad}^{2}$ & Added Mass Cross Term \\
$N_{p q}$ & $-4.86 e+000$ & $\mathrm{~kg} \cdot \mathrm{m}^{2} / \mathrm{rad}^{2}$ & Added Mass Cross-term \\
$N_{u u d r}$ & $-6.15 e+000$ & $\mathrm{~kg} / \mathrm{rad}$ & Fin Lift Moment \\
\hline \hline
\end{tabular}




\section{Chapter 5}

\section{Vehicle Tow Tank Experiments}

In April through June of 1999, the author collaborated with Ben Allen from WHOI's Oceanographic Systems Lab on a series of tow tank experiments with a full-scale REMUS vehicle [3]. These experiments were intended to measure the vehicle axial drag coefficient and the thrust of the vehicle propeller, and to assist in estimating the overall efficiency of the vehicle propulsion system. The experiments involved recording axial and lateral drag data for a range of vehicle speeds and hull configurations, as well as thrust data from bollard pull tests for a range of propeller speeds. These experiments provided the author with an opportunity to experimentally measure the vehicle axial drag coefficient.

\subsection{Motivation}

One of the more important attributes of any AUV is its endurance, or the range and speed that the vehicle has available to accomplish its mission. An increase in propulsion system efficiency corresponds to a longer range for a given speed, or the ability to cover the same distance in a reduced time. Any efforts to improve the overall efficiency will result in a more useful vehicle.

REMUS is a low-cost, man-portable AUV design with approximately 1000 hours of water time over hundreds of missions on 10 vehicles $[31,30]$. The vehicle design has been very successful in demonstrating the usefulness of AUVs in the ocean [28], however it is limited in its range and speed [2]. The existing design system used model airplane propellers with a DC brush motor, propeller shaft and shaft seal. A recent design effort entailed modifications to this design to provide significantly greater propulsion performance.

It is not possible to determine the difference between effects of hull drag coefficient and propeller efficiency in open water vehicle tests when neither the actual vehicle drag coefficient nor propeller efficiencies are known. Therefore the first step in the design process entailed quantifying the sources of drag in a tow-tank on an existing vehicle, and then determining what improvements were possible.

\subsection{Laboratory Facilities and Equipment}

The experiments were conducted at the University of Rhode Island Tow Tank, located in the Sheets Building on the Narragansett Bay Campus. The URI tow tank, which was filled with fresh water, is approximately 30 meters long by 3.5 meters wide by 1.5 meters deep (100 by 12 by 5 feet). The tow tank carriage had a useful run of almost 21 meters (70 feet). See Figure 5-1 for a diagram of the tow tank layout, and Figure 5-2 for a picture of the tank.

Given the large size of the tank relative to the vehicle, we were able to use an actual REMUS vehicle during the tests, rather than a scale model. The vehicle was suspended in the water by a 


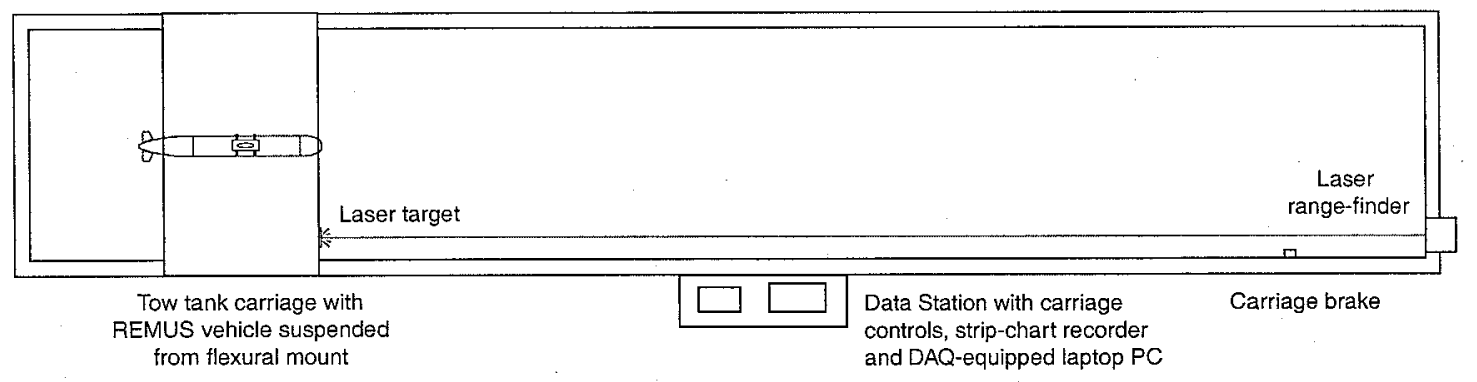

Figure 5-1: URI Tow Tank Layout

faired strut, which was connected to the towing carriage by the bottom plate of a flexural mount. See Figure 5-3 for a diagram of the carriage setup and vehicle mounting, and Figure 5-4 for a photo of the vehicle on the strut.

\subsubsection{Flexural Mount}

The flexural mount was a box consisting of two parallel, horizontal plates connected by flat vertical springs. The springs allowed the lower plate to move in the horizontal plane. The motion of plate relative to the carriage was measured with two orthogonally-mounted linear variable differential transformers (LVDTs), electromechanical transducers which converted the rectilinear motion of the plate along each horizontal axis into corresponding electrical signals.

The LVDT output signals were amplified and electronically filtered, then transmitted to the data station where they were plotted on a strip chart recorder and sampled by an analog-to-digital board connected to a laptop PC.

We were able to calibrate the axially-mounted LVDT to a significantly greater level of accuracy than the laterally-mounted instrument, due to the poor condition of the latter. As such, we only used the laterally-mounted LVDT for gross measurements of lateral drag, as an indicator of strut, vehicle or fin misalignment. s.

\subsubsection{Tow Tank Carriage}

The tow tank carriage was a large flat cart with hard rubber wheels driven by an electric motor. Instead of rails, the carriage rolled along the flat tops of the tank walls.

See Figure 5-5 for a picture of the tow tank carriage.

The desired carriage speed was set by a rheostat at the data station. A simple motor controller measured the carriage speed using an encoder wheel and light sensor mounted on the axle of the motor shaft. On forward runs, the carriage was stopped when a protruding trigger switch was thrown by a flange mounted on the tank wall. On backing up, the cart was stopped only by the alert operators stabbing at the motor kill switch mounted at the data station.

The speed at which we operated the carriage was limited more by the length of the tow tank than by the torque of the carriage motor. Our maximum speed was roughly 1.5 meters per second or 3 knots, the operating speed of the vehicle. At that velocity, the strut vibrations generated by the impulsive start took several seconds to damp out, leaving us with only a few seconds of useful data before the carriage began decelerating.

The actual carriage speed was measured using a laser range finder mounted at the far end of the tank. The range finder, a Nova Ranger NR-100, did not measure time-of-flight; instead, it was calibrated to measure distance based on the location of the projected dot. For a given distance, the instrument output a corresponding voltage. 


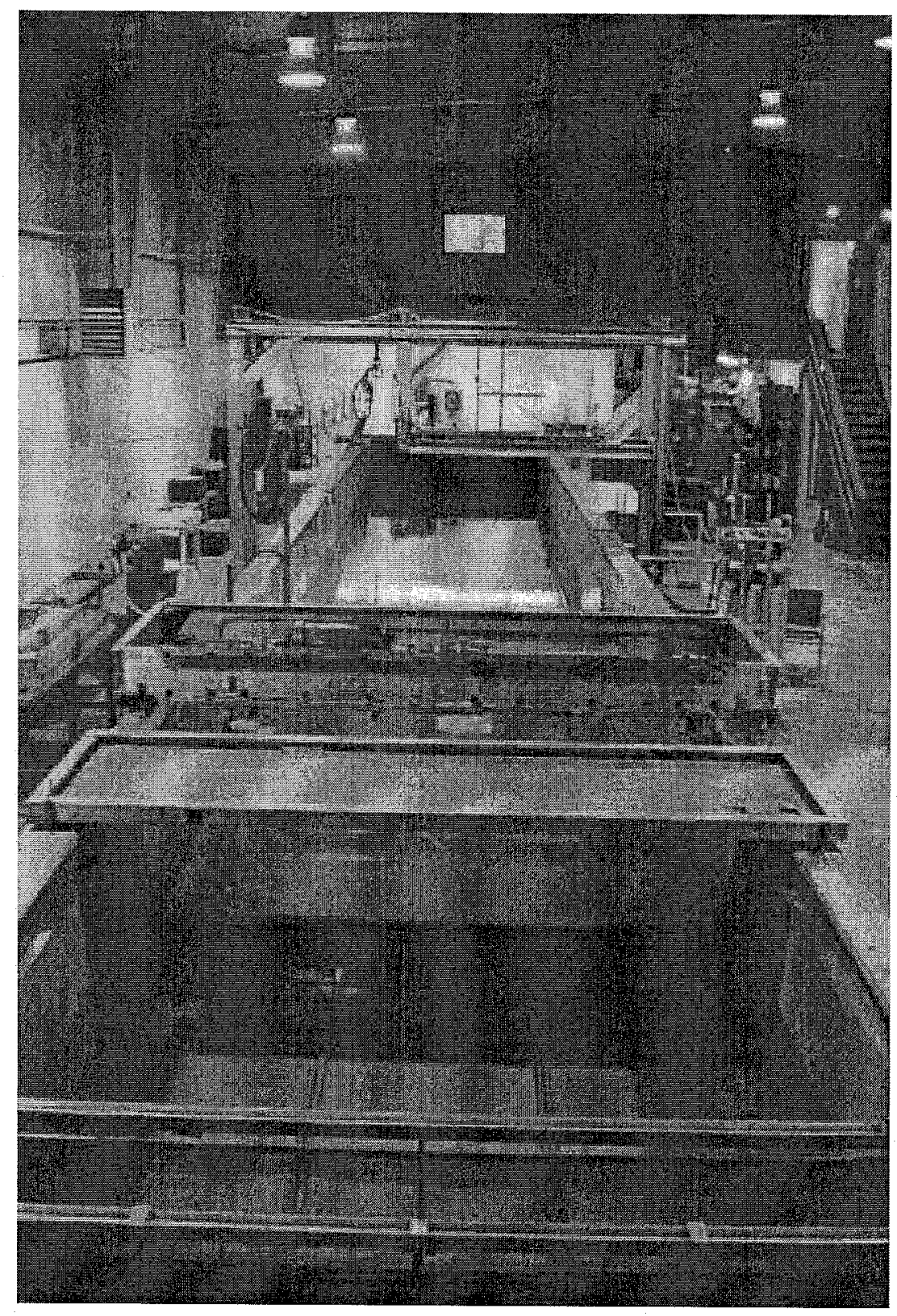

Figure 5-2: URI Tow Tank [Photo courtesy of URI Ocean Engineering Department] 


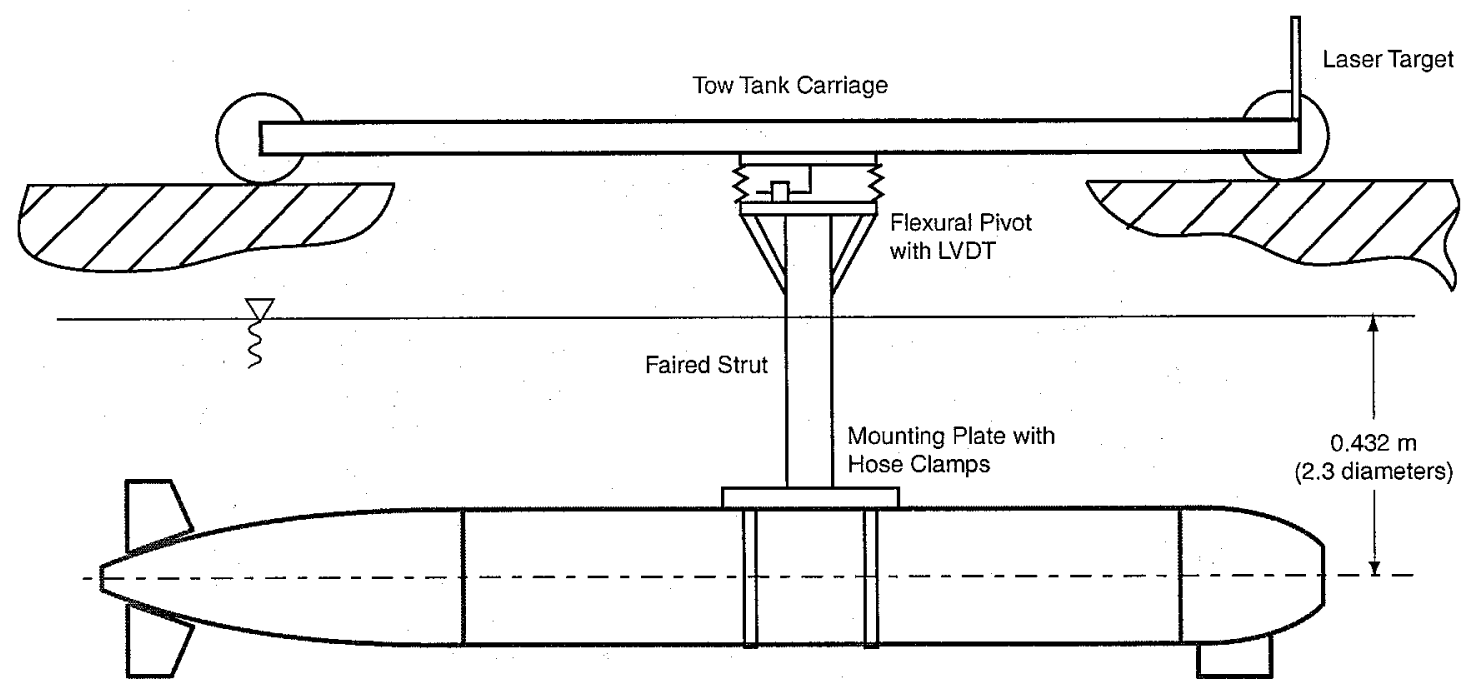

Figure 5-3: Carriage Setup and Vehicle Mounting

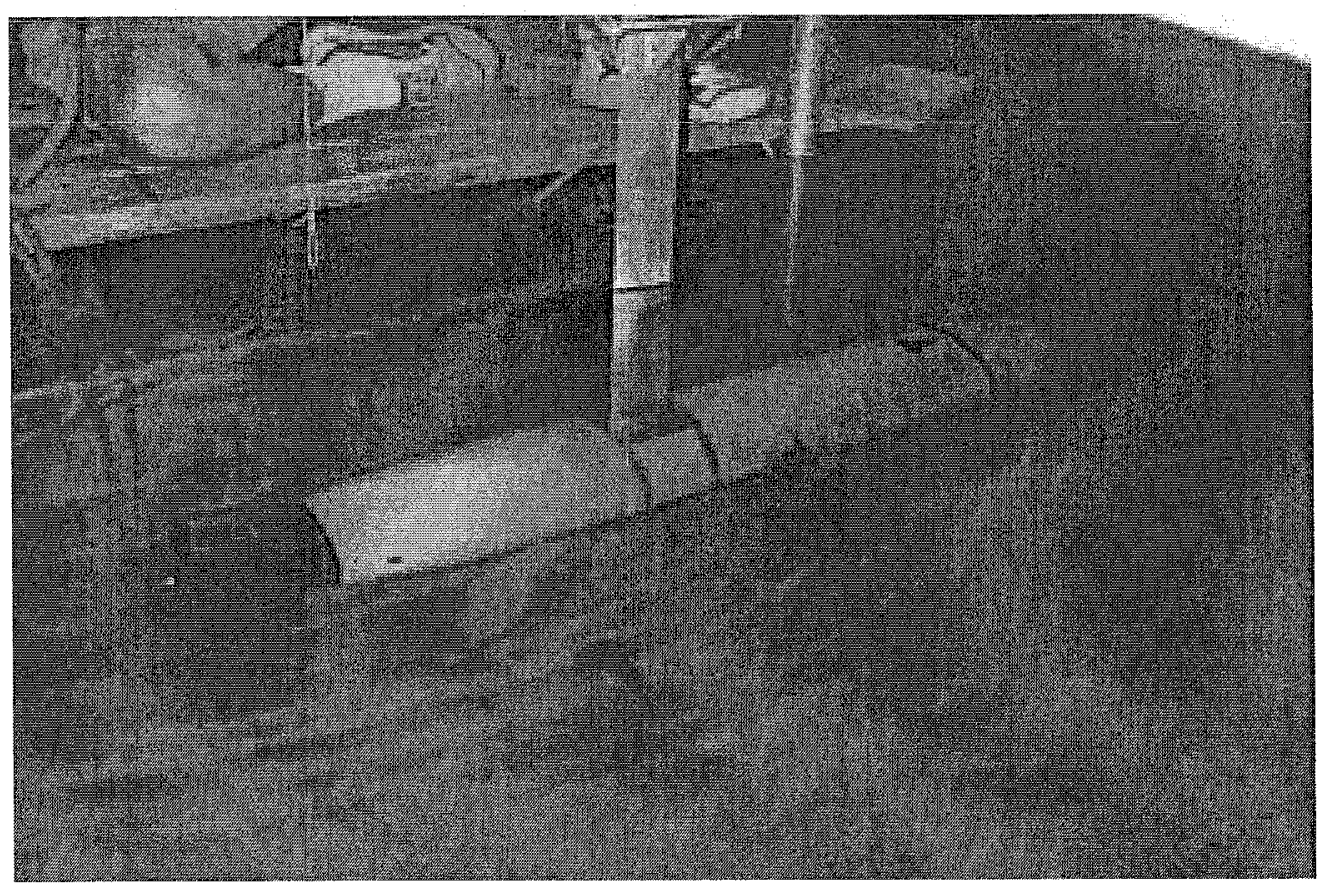

Figure 5-4: URI Tow Tank Carriage 


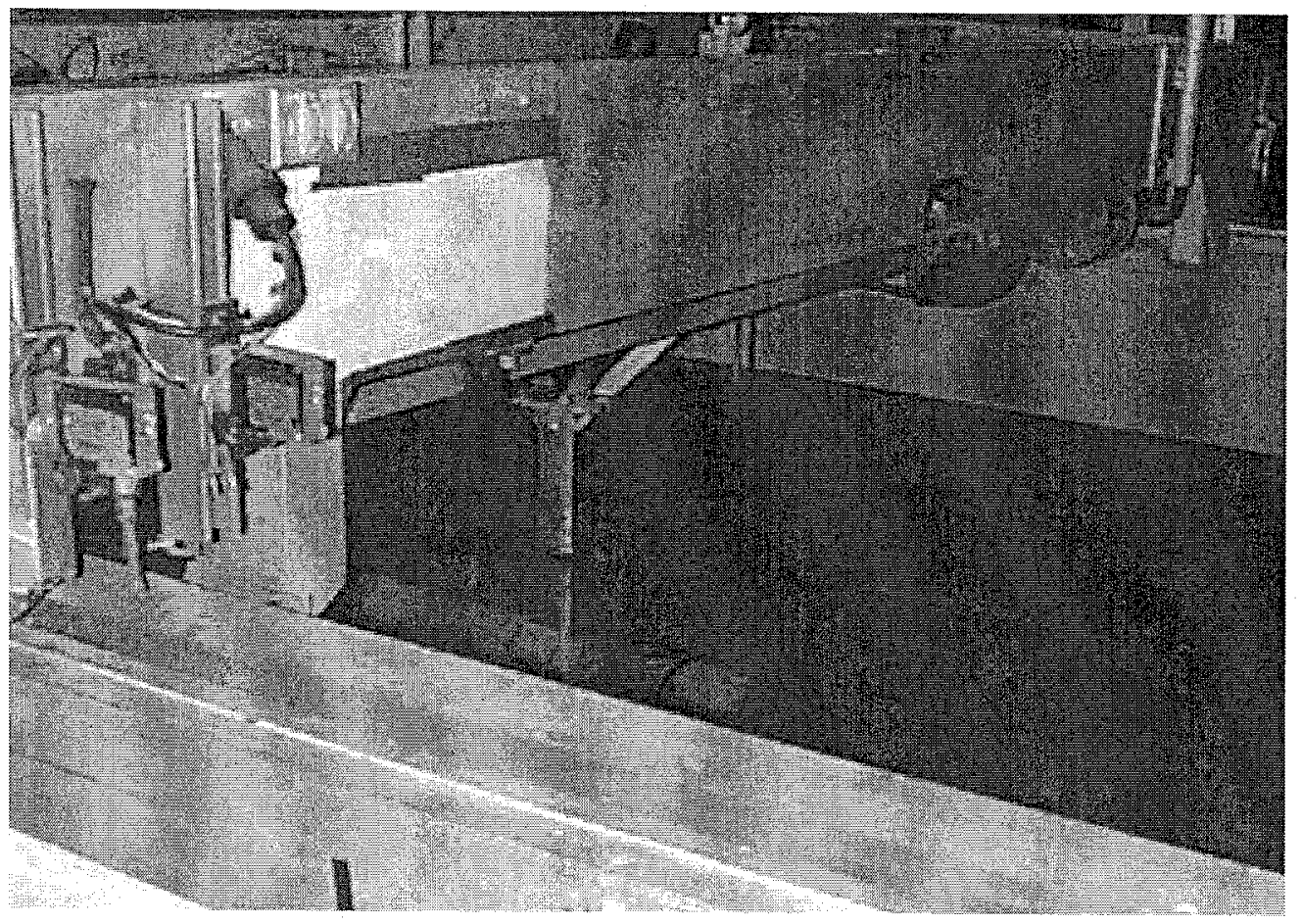

Figure 5-5: URI Tow Tank Carriage

The analog range finder signal was transmitted to the data station, where it too was sampled by the laptop PC's analog-to-digital board. Both digital signals were logged with data acquisition software, then processed with MATLAB.

\subsection{Drag Test Experimental Procedure}

The drag test experimental procedure involved the following steps:

- LVDT and strut pre-calibration

- vehicle mount and alignment check

- fin alignment check

- vehicle drag runs

- LVDT and strut post-calibration

\subsubsection{Instrument Calibration}

In calibrating the LVDT, we would apply a range of known loads to the flexural carriage and record the output voltage. This was accomplished by hanging weights from a line tied to the aft end of the bottom flexural plate and run over a pulley. Given that there was a small amount of friction in the LVDT shaft, after hanging the weights we would whack the flexural mount and allow the vibrations to damp out, recording the average steady value after several whacks. 
During the tank runs, we would periodically check the output voltage of the LVDT power supply, as the output of the aging instrument seemed to vary slightly as it warmed up.

In calibrating the strut, we would run the carriage through a range of speeds with just the bare strut in the water, recording the axial and lateral drag. If necessary, we would re-align the strut and run the test again. The measured strut drag as a function of carriage speed would later be subtracted from the total drag of each vehicle run, isolating the vehicle drag.

After performing the instrument calibrations and mounting the vehicle, we would check the vehicle the yaw alignment with a plumb bob, and vehicle pitch alignment by sighting through a window in the side of the tank.

In the initial experiments, we would check the alignment of the vehicle fins in a similar manner. Unfortunately, the fin drive chains on the WHOI1 tail section were both loose, so it was difficult to keep the fins aligned properly. We tried switching to a different tail section with tighter fins, but it was still difficult to sight the alignment of the lower rudder fin. In the end, we found it convenient to each day collect a data set with the fins removed, in order to verify the alignment of the vehicle.

\subsubsection{Drag Runs}

The tow tank runs were conducted at five different speeds between 0 and 1.5 meters per second. Between runs, we would begin processing the drag data while we waited for the waves in the tank to damp out.

After spending several sessions preparing the lab equipment and developing our calibration procedure, we ran four days of vehicle tests. Table 5.1 gives the dates and details of these experimental runs.

Table 5.1: REMUS Drag Runs

\begin{tabular}{ccl}
\hline \hline Date & Filename & \multicolumn{1}{c}{ Vehicle (notes) } \\
\hline 09 Jun 1999 & remxfps7 & WHOI1 \\
16 Jun 1999 & remdxfps8 & WHOI1 (DOCK2 tail) \\
16 Jun 1999 & remdxfps8b & WHOI1 (DOCK2 tail) \\
16 Jun 1999 & rnfdxfps8 & WHOI1 (DOCK2 tail, no fins) \\
16 Jun 1999 & rnfdxfps8b & WHOI1 (DOCK2 tail, no fins) \\
\hline \hline
\end{tabular}

\subsubsection{Signal Processing}

For a given run, we would collect data from three channels simultaneously-vehicle axial drag, vehicle lateral drag, and carriage speed-at a frequency of $400 \mathrm{~Hz}$ per channel. To remove sensor noise and the high-frequency strut and carriage vibrations, we filtered the data using a zero-phase forwards and reverse digital filter of order 250 and with a cut-off frequency of $2.5 \mathrm{~Hz}$. Figure 5-6 shows a comparison of the filtered and unfiltered data for a single channel.

\subsection{Experimental Results}

Figure 5-7 shows a plot of forward speed versus vehicle axial drag for the different configurations. These data were averaged to find a relationship between forward velocity and axial drag, based on the following formula:

$$
c_{d}=\frac{2 F_{d}}{\rho A_{f} v^{2}}
$$

where $F_{d}$ is the measured drag force (after subtraction of strut drag), $\rho$ the fluid density (999.1 $\left.\mathrm{kg} / \mathrm{m}^{3}\right), A_{f}$ the vehicle frontal area $(0.029$ meters $), v$ the measured vehicle forward velocity, and $c_{d}$ 


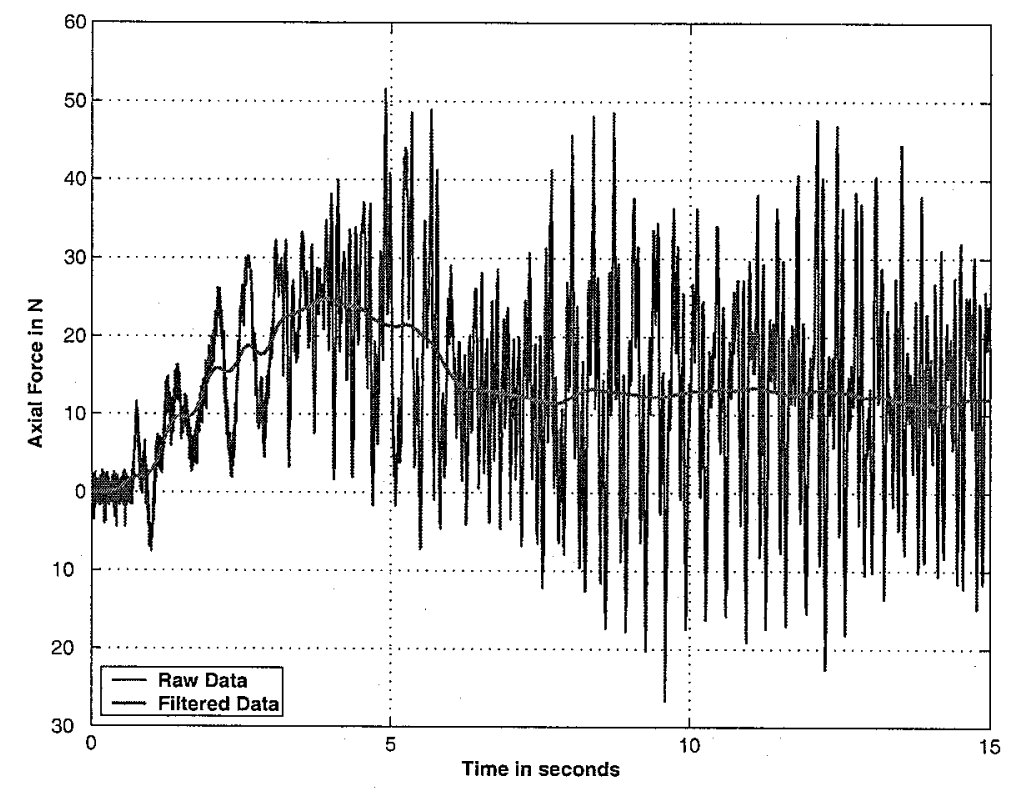

Figure 5-6: Vehicle Axial Drag. Carriage Speed $1.52 \mathrm{~m} / \mathrm{s}$. [remd5fps8b, 16 June 1999]

the vehicle drag coefficient. This resulted in an experimental average drag coefficient of 0.267 . The resulting parabolic fit is also plotted in Figure 5-7. Again, Table 5.1 gives the dates and details of these experimental runs.

Although the vehicle was towed at a depth of 2.3 body diameters, a significant amount of wavemaking was noticed in the tank for carriage speeds above one meter per second. This additional wave-making drag can be seen in Figure 5-7 as a deviation in the experimental data from the parabolic curve fit at higher carriage speeds.

\subsection{Component-Based Drag Model}

Bottaccini [7] and Hoerner [15] suggest a drag coefficient of 0.08 to 0.1 for torpedo shapes similar to REMUS, i.e. for fineness ratios (length over maximum diameter) of 6 to 11 . Given the experimentally-measured drag coefficient of 0.267 , it is obvious that the various hull protrusions contribute significantly to the total vehicle drag.

Table 5.2 lists the different vehicle components and their estimated contributions to the total vehicle drag. The drag coefficient value for the vehicle hull is from Myring [22] for a 'B' hull contour. The drag coefficient estimates for the vehicle components are taken from Hoerner [15]. All estimates assume a vehicle operating speed of 1.54 meters per second ( 3 knots). The resulting estimate for total drag yields, by Equation 5.1, an overall drag coefficient of 0.26, which compares well with the experimental results.

In Table 5.3, a similar component-based analysis is performed to predict the total drag of the sidescan sonar-equipped REMUS vehicle. 


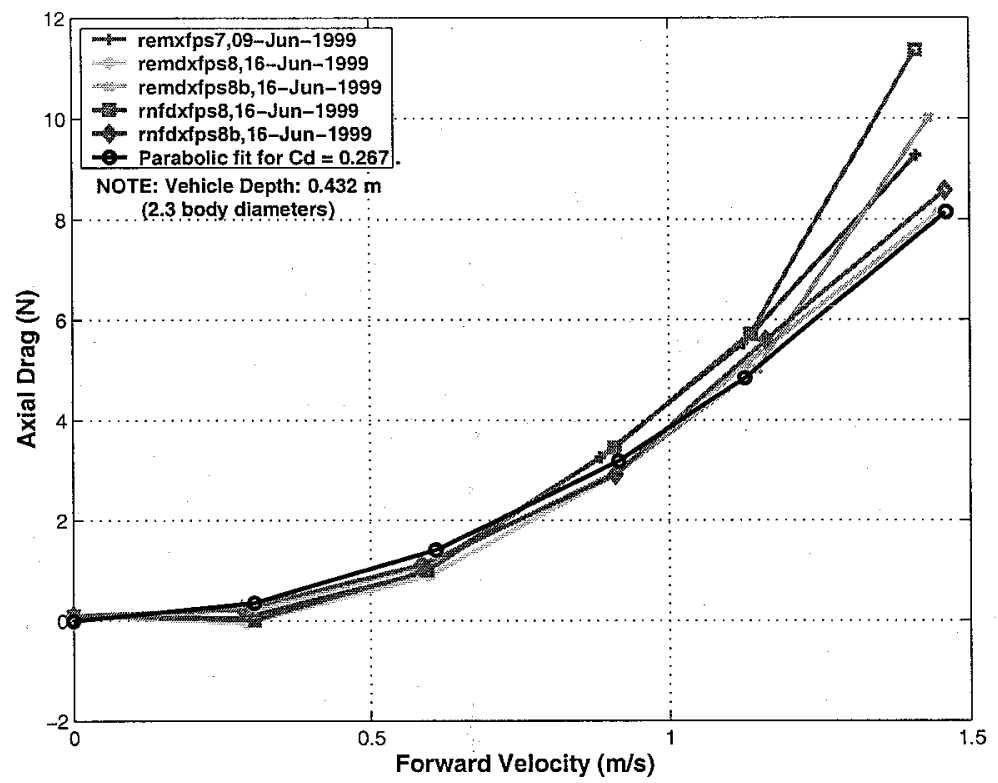

Figure 5-7: Forward Speed vs. Vehicle Axial and Lateral Drag (See Table 5.1 for experiment details)

Table 5.2: REMUS Component-Based Drag Analysis - Standard Vehicle

\begin{tabular}{rccccccc}
\hline \hline & Qty & $C_{d}$ & Length & Width & Diam. & Area & Drag \\
\cline { 5 - 8 } & & & $\mathrm{m}$ & $\mathrm{m}$ & $\mathrm{m}$ & $\mathrm{m}^{2}$ & $\mathrm{~N}$ \\
\hline Myring Hull & 1 & 0.10 & & & 0.19 & $2 . \mathrm{E}-04$ & 3.39 \\
Fins & 4 & 0.02 & 0.09 & 0.08 & & $5 . \mathrm{E}-05$ & 0.62 \\
LBL Transducer & 1 & 1.20 & 0.03 & 0.05 & & $1 . \mathrm{E}-05$ & 2.07 \\
Nose Pockets & 3 & 1.17 & & & 0.03 & $4 . \mathrm{E}-06$ & 2.68 \\
Blunt Nose & 1 & $?$ & & & & \\
& & & & \multicolumn{3}{r}{ Total Vehicle Drag: } & 8.77 \\
\cline { 5 - 8 } & & & & \multicolumn{3}{r}{ Effective Cd: } & 0.26 \\
\hline
\end{tabular}

Table 5.3: REMUS Component-Based Drag Analysis - Sonar Vehicle

\begin{tabular}{rccccccc}
\hline \hline & Qty & $C_{d}$ & Length & Width & Diam. & Area & Drag \\
\cline { 5 - 8 } & & & $\mathrm{m}$ & $\mathrm{m}$ & $\mathrm{m}$ & $\mathrm{m}^{2}$ & $\mathrm{~N}$ \\
\hline Myring Hull & 1 & 0.10 & & & 0.19 & $2 . \mathrm{E}-04$ & 3.39 \\
Fins & 4 & 0.02 & 0.09 & 0.08 & & $5 . \mathrm{E}-05$ & 0.62 \\
LBL Transducer & 1 & 1.20 & 0.03 & 0.05 & & $1 . \mathrm{E}-05$ & 2.07 \\
Nose Pockets & 3 & 1.17 & & & 0.03 & $4 . \mathrm{E}-06$ & 2.68 \\
Blunt Nose & 1 & $?$ & & & & & 0.00 \\
SSS Transducers & 2 & 0.40 & 0.04 & 0.04 & & $1 . \mathrm{E}-05$ & 1.47 \\
ADCP Transducers & 8 & 0.20 & & & 0.05 & $1 . \mathrm{E}-05$ & 3.86 \\
\hline & & & & \multicolumn{3}{r}{ Total Vehicle Drag: } & 14.09 \\
\cline { 5 - 7 } & & & & \multicolumn{3}{r}{ Effective Cd: } & 0.42 \\
\hline
\end{tabular}




\section{Chapter 6}

\section{Vehicle Simulation}

In this chapter, we begin by completing the equations governing vehicle motion. We then derive a numerical approximation for equations of motion and for the kinematic equations relating motion in the body-fixed coordinate frame to that of the inertial or Earth-fixed reference frame. Finally, we use that numerical approximation to write a computer simulation of the vehicle motion.

\subsection{Combined Nonlinear Equations of Motion}

Combining the equations for the vehicle rigid-body dynamics (Equation 3.8 ) with the equations for the forces and moments on the vehicle (Equation 4.49), we arrive at the combined nonlinear equations of motion for the REMUS vehicle in six degrees of freedom.

Surge, or translation along the $\mathrm{x}$-axis:

$$
\begin{gathered}
m\left[\dot{u}-v r+w q-x_{g}\left(q^{2}+r^{2}\right)+y_{g}(p q-\dot{r})+z_{g}(p r+\dot{q})\right]= \\
X_{H S}+X_{u|u|} u|u|+X_{\dot{u}} \dot{u}+X_{w q} w q+X_{q q} q q \\
+X_{v r} v r+X_{r r} r r+X_{\text {prop }}
\end{gathered}
$$

Sway, or translation along the y-axis:

$$
\begin{gathered}
m\left[\dot{v}-w p+u r-y_{g}\left(r^{2}+p^{2}\right)+z_{g}(q r-\dot{p})+x_{g}(q p+\dot{r})\right]= \\
Y_{H S}+Y_{v|v|} v|v|+Y_{r|r|} r|r|+Y_{\dot{v}} \dot{v}+Y_{\dot{r}} \dot{r} \\
+Y_{u r} u r+Y_{w p} w p+Y_{p q} p q+Y_{u v} u v+Y_{u u \delta_{r}} u^{2} \delta_{r}
\end{gathered}
$$

Heave, or translation along the z-axis:

$$
\begin{aligned}
m[\dot{w}-u q & \left.+v p-z_{g}\left(p^{2}+q^{2}\right)+x_{g}(r p-\dot{q})+y_{g}(r q+\dot{p})\right]= \\
& Z_{H S}+Z_{w|w|} w|w|+Z_{q \mid q q} q|q|+Z_{\dot{w}} \dot{w}+Z_{\dot{q}} \dot{q} \\
& +Z_{u q} u q+Z_{v p} v p+Z_{r p} r p+Z_{u w} u w+Z_{u u \delta_{s}} u^{2} \delta_{s}
\end{aligned}
$$

Roll, or rotation about the x-axis:

$$
\begin{gathered}
I_{x x} \dot{p}+\left(I_{z z}-I_{y y}\right) q r+m\left[y_{g}(\dot{w}-u q+v p)-z_{g}(\dot{v}-w p+u r)\right]= \\
K_{H S}+K_{p|p|} p|p|+K_{\dot{p}} \dot{p}+K_{\text {prop }}
\end{gathered}
$$


Pitch, or rotation about the y-axis:

$$
\begin{gathered}
I_{y y} \dot{q}+\left(I_{x x}-I_{z z}\right) r p+m\left[z_{g}(\dot{u}-v r+w q)-x_{g}(\dot{w}-u q+v p)\right]= \\
\quad M_{H S}+M_{w|w|} w|w|+M_{q|q|} q|q|+M_{\dot{w}} \dot{w}+M_{\dot{q}} \dot{q} \\
+M_{u q} u q+M_{v p} v p+M_{r p} r p+M_{u w} u w+M_{u u \delta_{s}} u^{2} \delta_{s}
\end{gathered}
$$

Yaw, or rotation about the z-axis:

$$
\begin{gathered}
I_{z z} \dot{r}+\left(I_{y y}-I_{x x}\right) p q+m\left[x_{g}(\dot{v}-w p+u r)-y_{g}(\dot{u}-v r+w q)\right]= \\
N_{H S}+N_{v|v|} v|v|+N_{r|r|} r|r|+N_{\dot{v}} \dot{v}+N_{\dot{r}} \dot{r} \\
+N_{u r} u r+N_{w p} w p+N_{p q} p q+N_{u v} u v+N_{u u \delta_{r}} u^{2} \delta_{r}
\end{gathered}
$$

We will find it convenient to separate the acceleration terms from the other terms in the vehicle equations of motion. The equations can thus be re-written as:

$$
\begin{aligned}
\left(m-X_{\dot{u}}\right) \dot{u} & +m z_{g} \dot{q}-m y_{g} \dot{r}=X_{H S}+X_{u|u|} u|u| \\
& +\left(X_{w q}-m\right) w q+\left(X_{q q}+m x_{g}\right) q^{2}+\left(X_{v r}+m\right) v r+\left(X_{r r}+m x_{g}\right) r^{2} \\
& -m y_{g} p q-m z_{g} p r+X_{\text {prop }} \\
\left(m-Y_{\dot{v}}\right) \dot{v} & -m z_{g} \dot{p}+\left(m x_{g}-Y_{\dot{r}}\right) \dot{r}=Y_{H S}+Y_{v|v|} v|v|+Y_{r|r|} r|r| \\
& +m y_{g} r^{2}+\left(Y_{u r}-m\right) u r+\left(Y_{w p}+m\right) w p+\left(Y_{p q}-m x_{g}\right) p q \\
& +Y_{u v} u v+m y_{g} p^{2}+m z_{g} q r+Y_{u u \delta_{r}} u^{2} \delta_{r} \\
\left(m-Z_{\dot{w}}\right) \dot{w} & +m y_{g} \dot{p}-\left(m x_{g}+Z_{\dot{q}}\right) \dot{q}=Z_{H S}+Z_{w|w|} w|w|+Z_{q|q| q}|q| \\
& +\left(Z_{u q}+m\right) u q+\left(Z_{v p}-m\right) v p+\left(Z_{r p}-m x_{g}\right) r p+Z_{u w} u w \\
& +m z_{g}\left(p^{2}+q^{2}\right)-m y_{g} r q+Z_{u u \delta_{s}} u^{2} \delta_{s} \\
-m z_{g} \dot{v} & +m y_{g} \dot{w}+\left(I_{x x}-K_{\dot{p}}\right) \dot{p}=K_{H S}+K_{p|p|} p|p| \\
& -\left(I_{z z}-I_{y y}\right) q r+m(u q-v p)-m z_{g}(w p-u r)+K_{\mathrm{prop}} \\
m z_{g} \dot{u}- & \left(m x_{g}+M_{\dot{w}}\right) \dot{w}+\left(I_{y y}-M_{\dot{q}}\right) \dot{q}=M_{H S}+M_{w|w|} w|w|+M_{q|q|} q|q| \\
& +\left(M_{u q}-m x_{g}\right) u q+\left(M_{v p}+m x_{g}\right) v p+\left[M_{r p}-\left(I_{x x}-I_{z z}\right)\right] r p \\
& +m z_{g}(v r-w q)+M_{u w} u w+M_{u u \delta_{s}} u^{2} \delta_{s} \\
-m y_{g} \dot{u} & +\left(m x_{g}-N_{\dot{v}}\right) \dot{v}+\left(I_{z z}-N_{\dot{r}}\right) \dot{r}=N_{H S}+N_{v|v|} v|v|+N_{r|r|}|r| \\
& +\left(N_{u r}-m x_{g}\right) u r+\left(N w p+m x_{g}\right) w p+\left[N_{p q}-\left(I_{y y}-I_{x x}\right)\right] p q \\
& -m y_{g}(v r-w q)+N_{u v} u v+N_{u u \delta_{r}} u^{2} \delta_{r}
\end{aligned}
$$

Finally, these equations can be summarized in matrix form as follows:

$$
\left[\begin{array}{cccccc}
m-X_{\dot{u}} & 0 & 0 & 0 & m z_{g} & -m y_{g} \\
0 & m-Y_{\dot{v}} & 0 & -m z_{g} & 0 & m x_{g}-Y_{\dot{r}} \\
0 & 0 & m-Z_{\dot{w}} & m y_{g} & -m x_{g}-Z_{\dot{q}} & 0 \\
0 & -m z_{g} & m y_{g} & I_{x x}-K_{\dot{p}} & 0 & 0 \\
m z_{g} & 0 & -m x_{g}-M_{\dot{w}} & 0 & I_{y y}-M_{\dot{q}} & 0 \\
-m y_{g} & m x_{g}-N_{\dot{v}} & 0 & 0 & 0 & I_{z z}-N_{\dot{r}}
\end{array}\right]\left[\begin{array}{c}
\dot{u} \\
\dot{v} \\
\dot{w} \\
\dot{p} \\
\dot{q} \\
\dot{r}
\end{array}\right]=\left[\begin{array}{c}
\sum X \\
\sum Y \\
\sum Z \\
\sum K \\
\sum M \\
\sum N
\end{array}\right]
$$

or

$$
\left[\begin{array}{c}
\dot{u} \\
\dot{v} \\
\dot{w} \\
\dot{p} \\
\dot{q} \\
\dot{r}
\end{array}\right]=\left[\begin{array}{cccccc}
m-X_{\dot{u}} & 0 & 0 & 0 & m z_{g} & -m y_{g} \\
0 & m-Y_{\dot{v}} & 0 & -m z_{g} & 0 & m x_{g}-Y_{\dot{r}} \\
0 & 0 & m-Z_{\dot{w}} & m y_{g} & -m x_{g}-Z_{\dot{q}} & 0 \\
0 & -m z_{g} & m y_{g} & I_{x x}-K_{\dot{p}} & 0 & 0 \\
m z_{g} & 0 & -m x_{g}-M_{\dot{w}} & 0 & I_{y y}-M_{\dot{q}} & 0 \\
-m y_{g} & m x_{g}-N_{\dot{v}} & 0 & 0 & 0 & I_{z z}-N_{\dot{r}}
\end{array}\right]^{-1}\left[\begin{array}{c}
\sum X \\
\sum Y \\
\sum Z \\
\sum K \\
\sum M \\
\sum N
\end{array}\right]
$$




\subsection{Numerical Integration of the Equations of Motion}

The nonlinear differential equations defining the vehicle accelerations (Equation 6.9) and the kinematic equations (Equations 3.1 and 3.4) give us the vehicle accelerations in the different reference frames. Given the complex and highly non-linear nature of these equations, we will use numerical integration to solve for the vehicle speed, position, and attitude in time.

Consider that at each time step, we can express Equation 6.9 as follows:

$$
\dot{x}_{n}=f\left(x_{n}, u_{n}\right)
$$

where $\boldsymbol{x}$ is the vehicle state vector:

$$
x=\left[\begin{array}{llllllllllll}
u & v & w & p & q & r & x & y & z & \phi & \theta & \psi
\end{array}\right]^{T}
$$

and $\boldsymbol{u}_{n}$ is the input vector:

$$
\boldsymbol{u}_{n}=\left[\begin{array}{llll}
\delta_{s} & \delta_{r} & X_{\text {prop }} & K_{\text {prop }}
\end{array}\right]^{T}
$$

Refer back to Section 3.2 for the definitions of the vehicle states and inputs, and to Figures 4-1 and 4-2 for the fin angle sign conventions.

The following sections summarize three methods of numerical integration in order of increasing accuracy.

\subsubsection{Euler's Method}

We will first consider Euler's method, a simple numerical approximation which consists of applying the iterative formula:

$$
x_{n+1}=x_{n}+f\left(x_{n}, u_{n}\right) \cdot \Delta t
$$

where $\Delta t$ is the modeling time step. Euler's method, although the least computationally intensive method, is unacceptable as it can lead to divergent solutions for large time steps.

\subsubsection{Improved Euler's Method}

The following method improves the accuracy of Euler's method by averaging the tangent slope for two points along the line. We first calculate the following:

$$
\begin{aligned}
& k_{1}=x_{n}+f\left(x_{n}, u_{n}\right) \cdot \Delta t \\
& k_{2}=f\left(k_{1}, u_{n+1}\right)
\end{aligned}
$$

And then combine them to calculate the new state vector:

$$
x_{n+1}=x_{n}+\frac{\Delta t}{2}\left(f\left(x_{n}, u_{n}\right)+k_{2}\right)
$$

This method is significantly more accurate than Euler's method. 


\subsubsection{Runge-Kutta Method}

This method further improves the accuracy of the approximation by averaging the slope at four points. We first calculate the following:

$$
\begin{aligned}
& k_{1}=x_{n}+f\left(x_{n}, u_{n}\right) \\
& k_{2}=f\left(x+\frac{\Delta t}{2} k_{1}, u_{n+\frac{1}{2}}\right) \\
& k_{3}=f\left(x+\frac{\Delta t}{2} k_{2}, u_{n+\frac{1}{2}}\right) \\
& k_{4}=f\left(x+\Delta t k_{3}, u_{n+1}\right)
\end{aligned}
$$

where the interpolated input vector

$$
\boldsymbol{u}_{n+\frac{1}{2}}=\frac{1}{2}\left(\boldsymbol{u}_{n}+u_{n+1}\right)
$$

We combine the above equations to yield:

$$
x_{n+1}=x_{n}+\frac{\Delta t}{6}\left(k_{1}+2 k_{2}+2 k_{3}+k_{4}\right)
$$

This method is is the most accurate of the three. This is what we shall use in the vehicle model code.

\subsection{Computer Simulation}

As described in the Introduction, the author implemented this numerical approximation using MATLAB. The model code can be seen in Appendix E. The model code works by calculating for each time step the forces and moments on the vehicle as a function of vehicle speed and attitude. These forces determine the vehicle body-fixed accelerations and earth-relative rates of change. These accelerations are then used to approximate the new vehicle velocities, which become the inputs for the next modeling time step.

The vehicle model requires two inputs:

- Initial conditions, or the starting vehicle state vector.

- Control inputs, or the vehicle pitch fin and stern plane angles, either given as a pre-determined vector, when comparing the model output with field data, or calculated at each time step, in the case of control system design. 


\section{Chapter 7}

\section{Field Experiments}

\subsection{Motivation}

In order to verify the accuracy of the vehicle model, the author conducted a series of experiments at sea measuring the response of the vehicle to step changes in rudder and stern plane angle. These experiments were conducted with the assistance of the Oceanographic Systems Lab staff at both the Woods Hole Oceanographic Institution and at the Rutgers University Marine Field Station in Tuckerton, New Jersey.

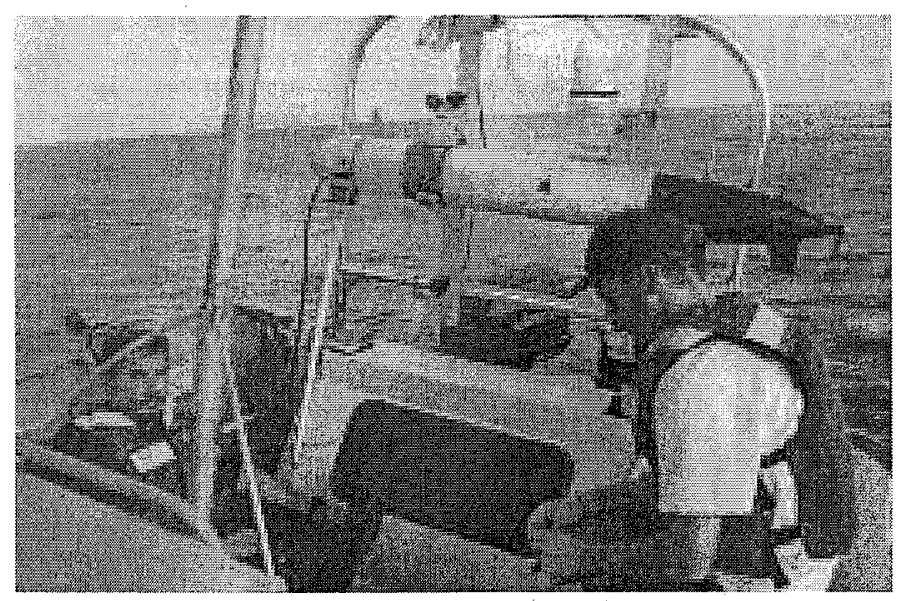

Figure 7-1: The author (left) and Mike Purcell from WHOI OSL, running vehicle experiments at the Rutgers Marine Field Station in Tuckerton, NJ [Photo courtesy of Nuno Cruz, Porto University]

\subsection{Measured States}

In each experiment at sea we measured the vehicle depth and attitude, represented in the vehicle model by the following, globally-referenced vehicle states:

$$
\boldsymbol{x}=\left[\begin{array}{llll}
z & \phi & \theta & \psi
\end{array}\right]^{T}
$$


In these experiments we also recorded the vehicle fin angles, represented in the vehicle model by the following vehicle-referenced control inputs:

$$
\boldsymbol{u}_{n}=\left[\begin{array}{ll}
\delta_{s} & \delta_{r}
\end{array}\right]^{T}
$$

Refer to Figure 3-1 for a diagram of the vehicle coordinate system, and to Figures 4-1 and 4-2 for diagrams of the control fin sign conventions.

Note that for all of the field tests described in this section, the vehicle propeller was not used as a control input, but was instead kept at a constant 1500 RPM. As propeller thrust and torque were difficult to estimate for different propeller RPMs, sticking to a constant value allowed us to remove a source of uncertainty from the vehicle model comparison.

\subsection{Vehicle Sensors}

The following were the navigation sensors available during the author's field experiments. For each sensor, we will give the sensor's function, and its known limitations.

Note that sensor accuracy is often a function of cost. Vehicles like REMUS are designed to be relatively inexpensive- a high precision gyro-compass, for example, might double the cost of the vehicle. The challenge in vehicle design is to identify the least expensive sensor suite that meets the vehicle's navigation requirements.

\subsubsection{Heading: Magnetic Compass}

Vehicle heading was measured with a triaxial fluxgate magnetometer, which senses the orientation of the vehicle with respect to earth's magnetic vector. The magnetometer is sensitive to magnetic noise, such as is generated by the various electronic components within the vehicle housing. The calibration routine for this sensor has the vehicle drive in circles while pitching and rolling-by integrating the yaw rate and comparing it with the measured heading, a table of compass deviation as a function of vehicle heading can be made.

This magnetic calibration can correct for constant sources of magnetic noise, such as the vehicle batteries, but not for intermittent signals such as the fin and propeller motors. As a result, heading measurements can be off by as much as five degrees.

\subsubsection{Yaw Rate: Tuning Fork Gyro}

Vehicle yaw rate is measured with a tuning fork gyroscope. The integral of the sensor output to obtain heading is vulnerable to drift, and is therefore more accurate when measuring high frequency vehicle motions. By combining the low-pass filtered compass data with high-pass filtered and integrated yaw rate gyro data, we can arrive at a more accurate estimate for the vehicle heading.

\subsubsection{Attitude: Tilt Sensor}

Vehicle pitch and roll are measured with an electrolytic tilt and roll sensor. This sensor measures the position of a blob of conducting fluid in a cup. For example, the vehicle pitching down is indicated by the fluid sloshing forward.

This sensor is accurate for low-frequency motion, but will obviously: have problems capturing high frequency motion due to the inertia of the conducting fluid. Furthermore, the motion of the fluid is coupled such that high vehicle yaw rates or surge accelerations give false pitch measurements. 


\subsubsection{Depth: Pressure Sensor}

The vehicle depth is measured by a pressure sensor. This instrument is somewhat sensitive to changes in the surrounding sea water temperature, but its errors are small in magnitude relative to the error in the compass and attitude sensors.

\subsection{Experimental Procedure}

In this section, we describe the procedure used in the field experiment listed in Table 7.1. These experiments were run in roughly ten meters of water, both in Hadley's Cove near the Woods Hole Oceanographic Institution, and off the Atlantic coast near the Rutgers Marine Field Station in Rutgers, New Jersey.

Table 7.1: Vehicle Field Experiments

\begin{tabular}{cccl}
\hline \hline Date & Filename & Vehicle & Location \\
\hline 29 Jul 1998 & d980729a & STD REMUS (Dock1) & RUMFS \\
29 Jul 1998 & d980729b & STD REMUS (Dock1) & RUMFS \\
27 Oct 1998 & d981027 & STD REMUS (Dock1) & Hadley's \\
28 Oct 1998 & d981028 & STD REMUS (Dock1) & Hadley's \\
26 Jul 1999 & a990726 & NSW REMUS (NSW) & RUMFS \\
27 Jul 1999 & a990727 & NSW REMUS (NSW) & RUMFS \\
\hline \hline
\end{tabular}

\subsubsection{Pre-launch Check List}

Before each mission, the author ran through the checklist shown in Figure 7-2 to check the vehicle housing seals, and to verify operation of the vehicle sensors and communications.

\subsubsection{Trim and Ballast Check}

Following the pre-launch checklist, the author weighted the vehicle and measured the longitudinal center of gravity, $x_{c g}$, on a balance. The vehicle buoyancy was measured in a sea water tank, and the vehicle ballast adjusted to achieve 1.5 pounds of positive buoyancy as described in Section 2.4 .

\subsubsection{Vehicle Mission Programming}

The REMUS vehicle uses a component-based mission programming architecture. Each element in the mission is called an objective. The following types of objectives were used in the thesis experiments:

- SET POSITION: This command gives the vehicle its starting position as a range and bearing from a given latitude and longitude.

- WATT PROP: This command tells the vehicle to remain on standby until it detects the given propeller RPM. This allows us to start the vehicle mission by reaching into the water and spinning the vehicle propeller. The mission program starts, the propeller starts spinning on its own, we push the vehicle underwater and it is on its way.

- LONG BASELINE: This command tells the vehicle to navigate to the given latitude and longitude, using the given transponder beacons. In this mode, the vehicle uses long baseline navigation, dead-reckoning its position between acoustic fixes. See Roger Stokey's paper [28] for details on REMUS navigation. 


\section{THESIS EXPERIHENT PRE-LAUNCH CHECKLIST}

MISIONOBJ ESTHE

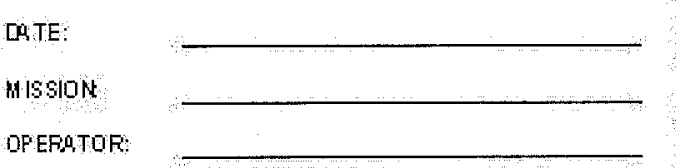

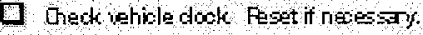

Q Thed that the OES E worling

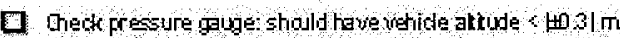

0 Ged tha CTOdata ndies senee in ar

bono Temp: Aes:

a Fecord onpass fhoh and nd offest:

Ath: Foll:

0 Thed ADCP operation

Listen for tanstitson fings Eventiles beirg colected in tenturidow.

Q Theck ocoustes on berioh

Hioomment ATS dagnostios in mes on file

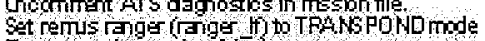
Aace tariduors 1 to 2 inches ad

Diect numbers:

SN> 7000

attonutios 45 ([nop os wten nemoned]

IBSN 6

Pe $+>2000$

Tum RAliGg of, \&enty otenuacor drope bebu $x$

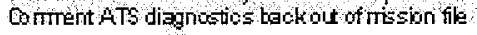

Q Thed torews or oflasas bird-ina e connetre mointing plate

¿ Ghed llul seril and power ooninetons, tewrap ompass serialounnotor.

Q Verify RBulusrangs operation on 5 seonds.

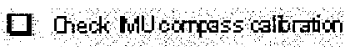
ASCl cormends though serial de Eug:

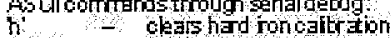
hat - stope poling

$\mathrm{bo}^{\prime}-$ dears ompassoa retart - retatsoompass

a Gect Muloperation $n$ headrig ptoh, and roll Compass light GREAN?

Q Thed $9 /$ sorer back up batton nottage. Peplace if necesay.

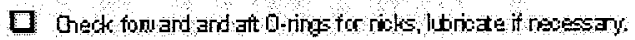

$\square$ bte ADutail section:

\begin{tabular}{|c|c|}
\hline & REHUS, HOUSMG CLOSED \\
\hline 0 & Qhed mith dy housing fon and and aft tor pineted 0 -nitgs \\
\hline$\square$ & 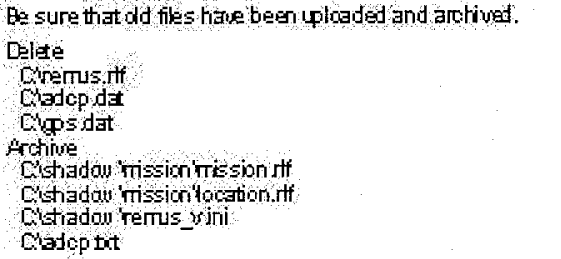 \\
\hline$\square$ & 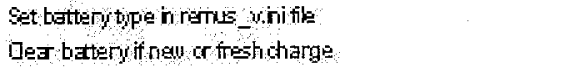 \\
\hline
\end{tabular}

G yenfy RBUUS range abort. Weke sire the prop is clear, therisend five pings

Q set detarde to maxium nrenuses ini Set Logevernth tatus $m g^{\prime \prime}=1$

0 Ged pith ard rudde fin algiments Beord offes from remus y iní

Ftch:

Fudder:

D. Fogram LOCATIUN tik.

Triple efeek all bury LATLONG, be au are of offeet and abuas tee the tue Eubng lootiors indisted in the LOCATION INFO pull doun.

D ForamMss okfik

(2) Thed mission usng FOUTEINFO. Wrif OETH Ltanca and Filfor eschkg: Vinftata tire of nission

] Ges al taut resoges.

$\square$ FESTART Ueticle.

a. Giedt that al light: are GRER Fead any falt mes age that apper.

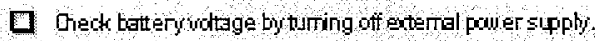
Shoud be $\geq 24$ bit Fecord battorinoltoge:

Figure 7-2: REMUS Pre-Launch Checklist (Page One) 
- TIMER: This command tells the vehicle to maintain the given depth or heading using feedback control, or to maintain a given, fixed fin angle or propeller RPM. The timer commands represent the experimental sections of each mission.

These objectives are edited and sent to the vehicle as a text files. See Appendix F for an example mission file.

In order to measure the vehicle response to step changes in fin angle, the vehicle was given the following commands:

- Timer to desired depth For "pitch up", the vehicle was commanded to six meters depth, to avoid breaking the surface. For "pitch down" commands, the vehicle was commanded to 2 meters depth.

- Step change in fin angle Upon achieving depth, the vehicle was commanded to hold a certain fin angle for two seconds in the case of vertical plane response, or longer for horizontal plane response.

The fin angle duration of two seconds was chosen as a result of the experimental run shown in Figure 7-3. In the depth plot right around the seven-second mark, you can see that the vehicle ran. into and bounced off the bottom. Given the unpredictable vehicle open-loop response, the author thought it wise to use short periods.

\subsubsection{Compass Calibration}

As described in Section 7.3.1, it was periodically necessary to update the vehicle compass calibration. The compass calibration objective could be included at the start of any mission file.

\subsubsection{Vehicle Tracking}

During the mission, the vehicle was tracked using a sonar transponder. See Figure 7-5 for a photo of the tracking equipment.

At the end of the mission, the vehicle would be recovered from the surface, and reprogrammed and relaunched if necessary.

\subsection{Experimental Results}

From these vehicle experiments, we get measurements for the vehicle response to temporary step changes in rudder and stern plane angle. It is important to note that during straight and level flight, the vehicle operates at a roll offset of negative five degrees $(\phi=-5)$ due to the propeller torque. As a result, we never get pure vertical- or horizontal-plane motion. That said, the vehicle roll is small enough that we are still able identify the vehicle behavior in pitch and yaw.

See Figure 7-6 for REMUS motion while operating under closed-loop control, for comparison with the open-loop, step response data. In the example shown, the vehicle was commanded to maintain a depth of two meters.

\subsubsection{Horizontal-Plane Dynamics}

The vehicle response to a step change in rudder angle is shown in Figure 7-7. For the objective shown, the rudder fin was fixed at four degrees, and the vehicle was commanded to maintain constant depth through closed-loop control..

The relevant information in this set of plots is that, for a rudder angle $\left(\delta_{r}\right)$ of roughly four degrees, the vehicle yaw rate was approximately 10 degrees/second. 

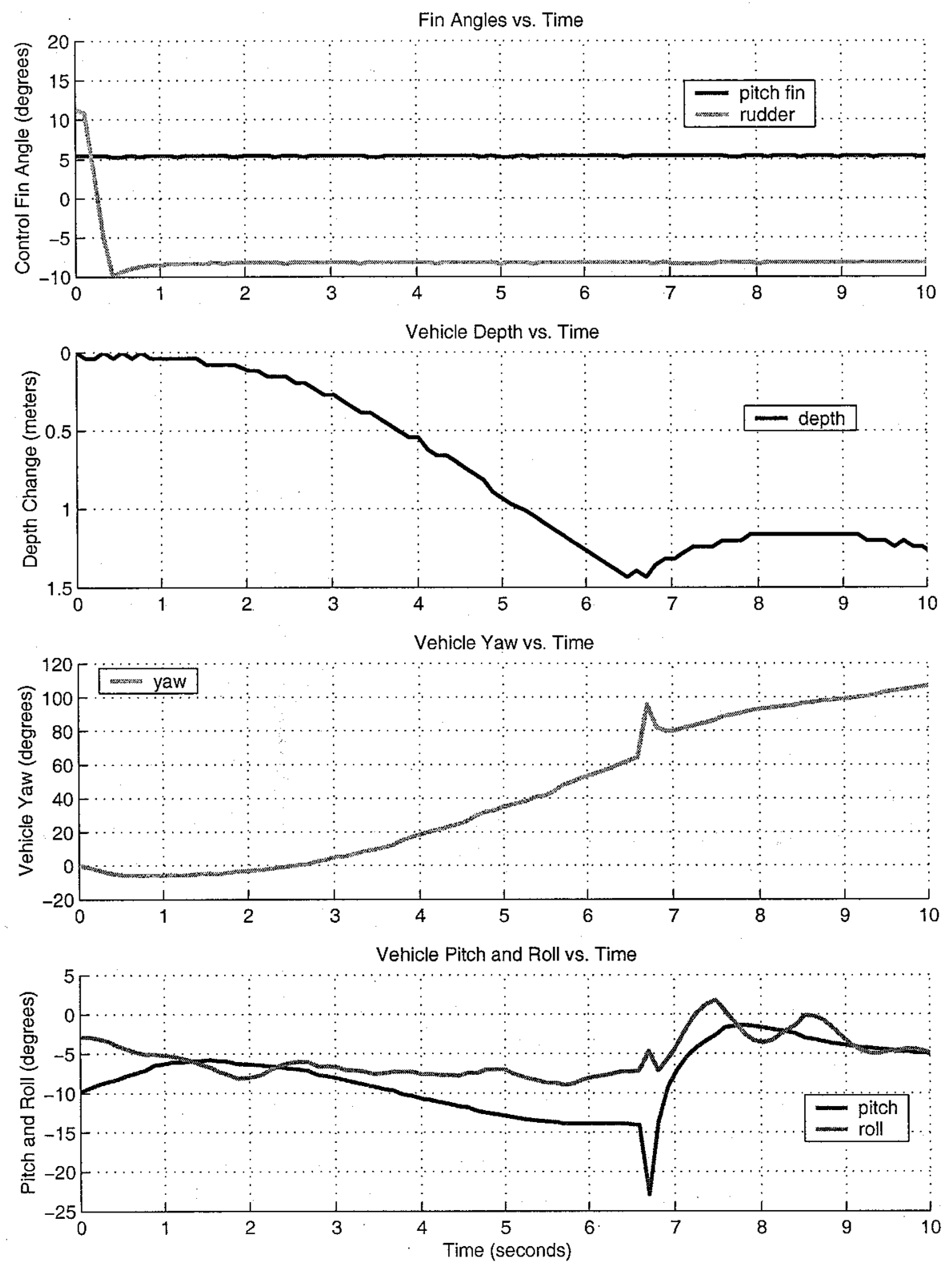

Figure 7-3: REMUS Mission Data: Vehicle bounces off the bottom [d980729a, Obj. 6 ] 

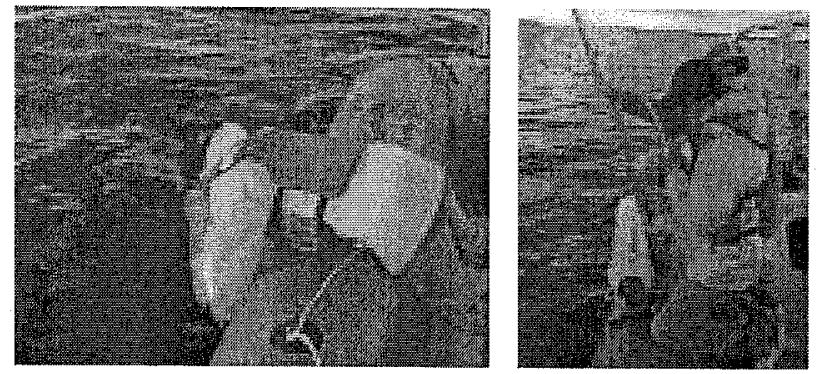

Figure 7-4: Launching and recovering the REMUS vehicle. [Photo courtesy of Rob Goldsborough, WHOI $O S L]$

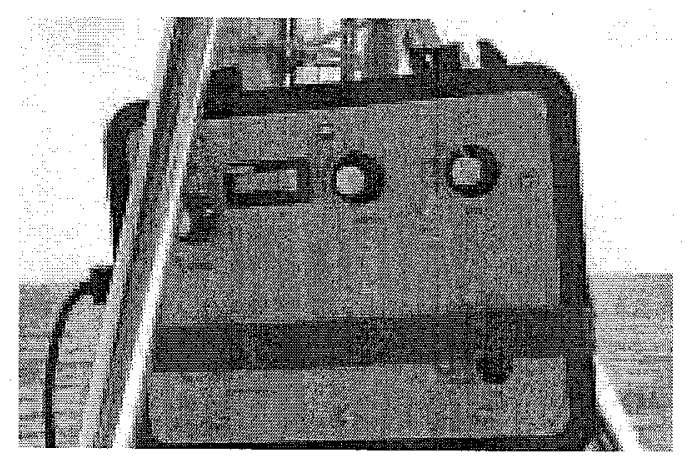

Figure 7-5: The REMUS Ranger

\subsubsection{Vertical-Plane Dynamics}

Figures 7-8 and 7-9 show the vehicle response to different temporary step changes in fin angle. In both cases, the vehicle was operating under closed-loop depth control until the step change command was given. The time scale on each plot has been shifted such that the step change command occurs at time $t=2$ seconds.

In Figure 7-8, the vehicle pitch fin (stern plane) angle $\delta_{s}$ was fixed at negative two degrees. The vehicle is seen to rise roughly 0.5 meters, and that the pitch change is roughly 20 degrees. At the end of the interval, both the depth and pitch rates were increasing. The vehicle is show to have a slightly negative depth rate (rising at roughly 0.5 meters per second) at the instant of the fin step change.

In Figure 7-9, the vehicle pitch fin angle $\delta_{s}$ was fixed at eight degrees. The vehicle is seen to dive roughly 0.4 meters, and the pitch change is roughly 18 degrees. Again, at the end of the interval, both the depth and pitch rates were increasing.

Also in Figure 7-9, the vehicle is shown to require a fin angle of positive four degrees in order to maintain a constant depth in the first two seconds. This suggests that the vehicle was ballasted slightly nose-down. This may be due to internal ballast weights shifting during the launch of the vehicle.

Despite the fact that the vehicle was operating under closed-loop heading control at all times, you will notice some heading drift in the data. It is not clear whether this reflects actual vehicle motion, or instrument error. 

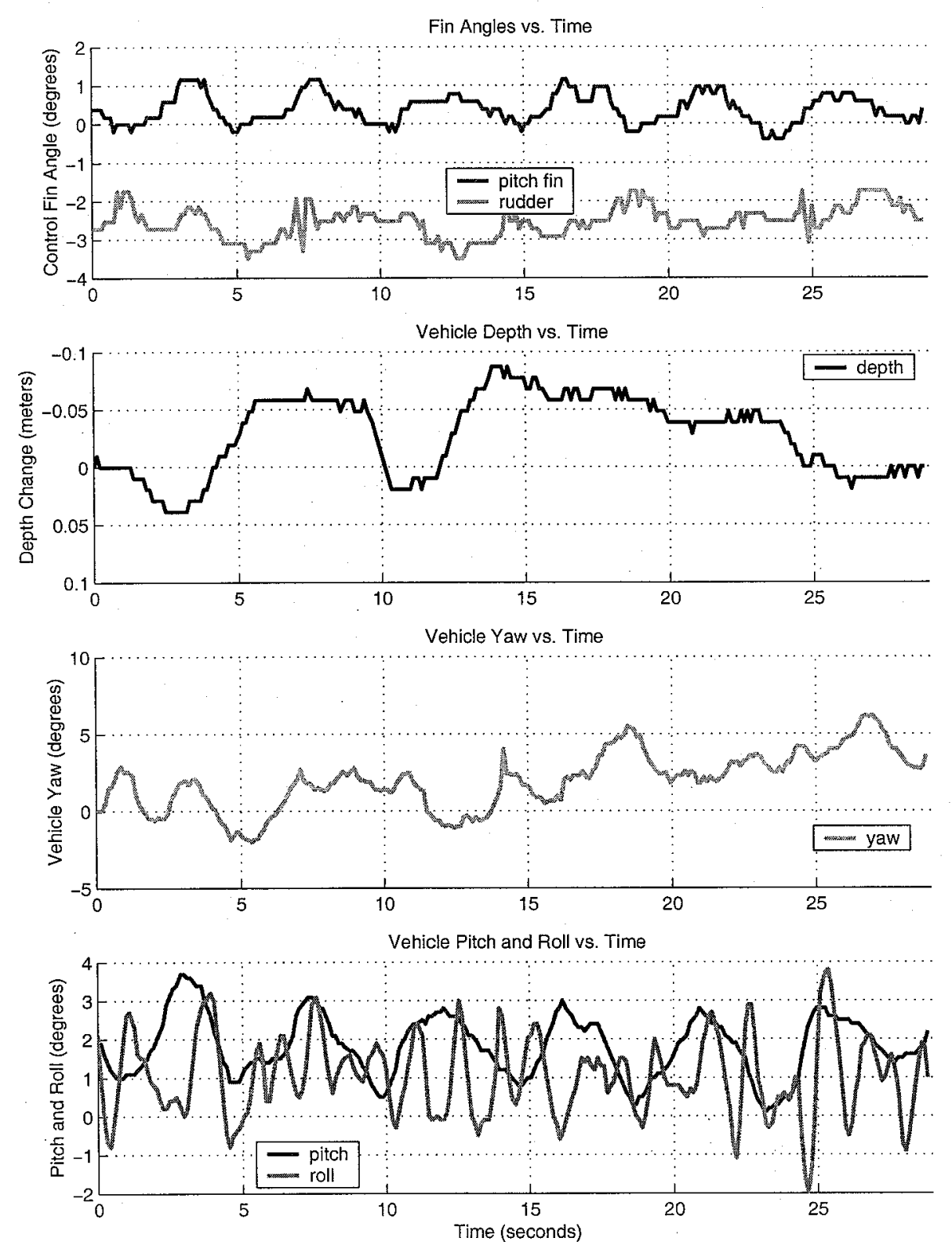

Figure 7-6: REMUS Mission Data: Vehicle under closed-loop control. [d990727, Obj. 4] 

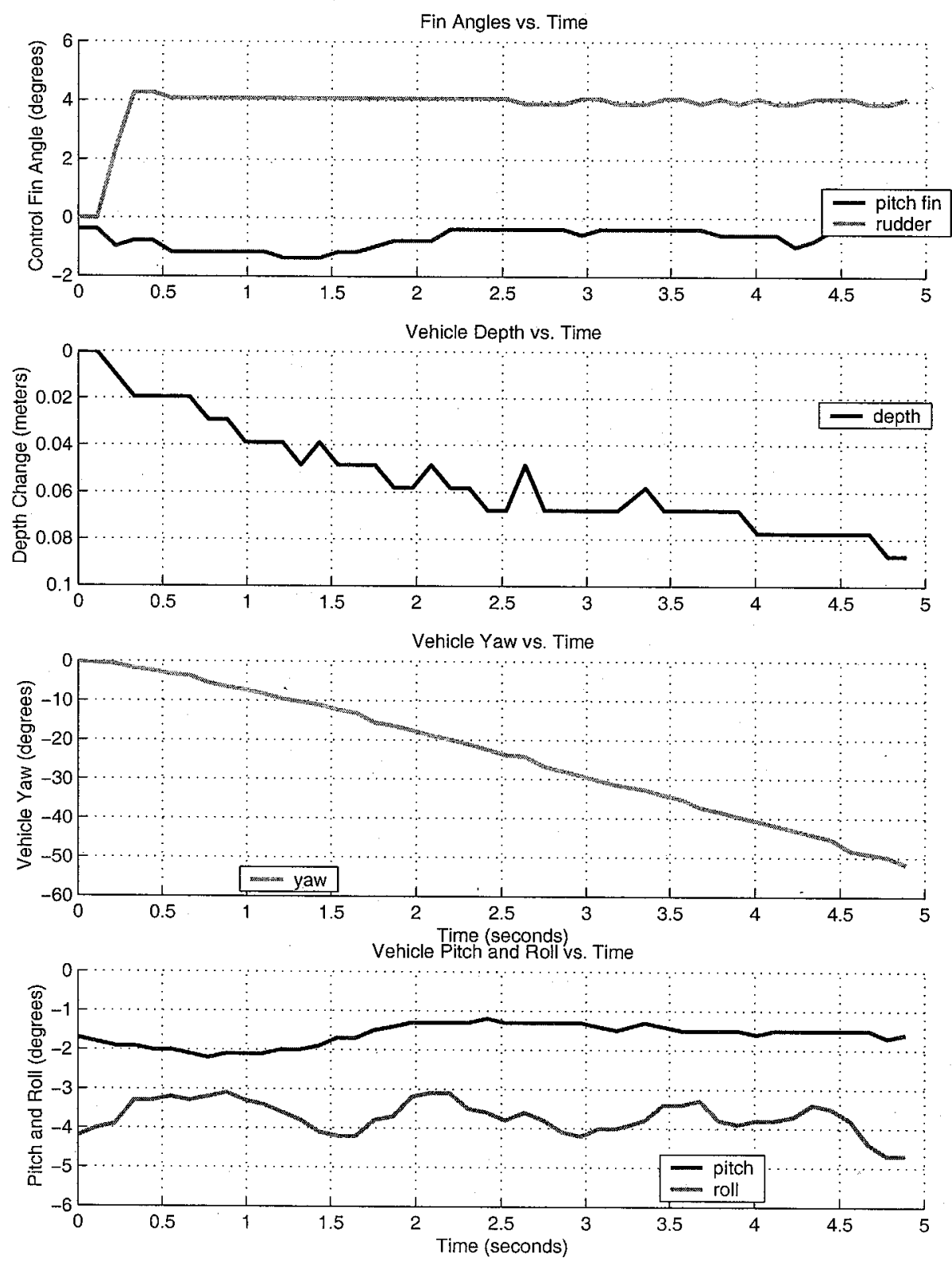

Figure 7-7: REMUS Mission Data: Step change in rudder angle. [d981028, Obj. 25] 

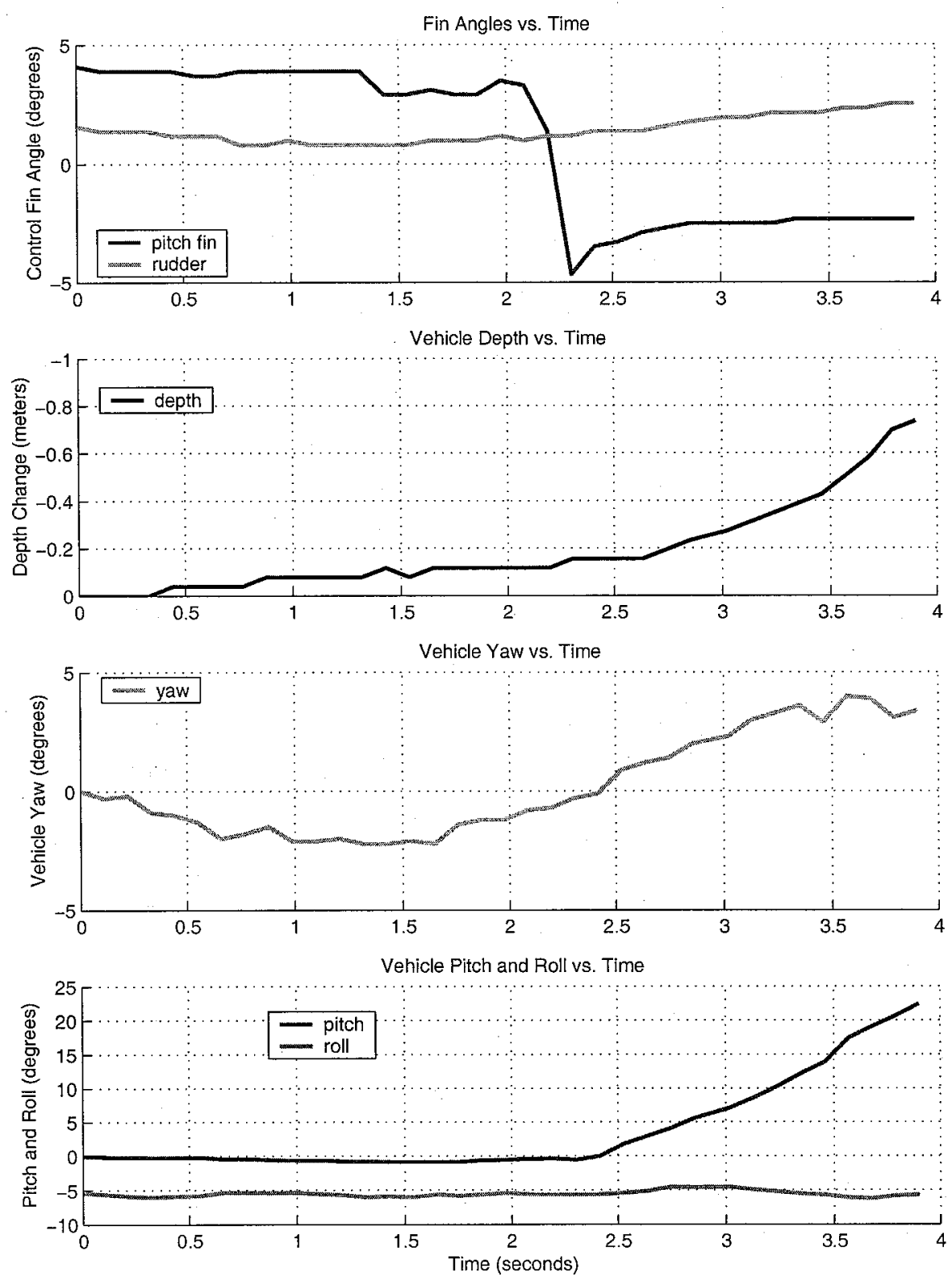

Figure 7-8: REMUS Mission Data: Vehicle pitching up. [d980729b, Obj. 14] 

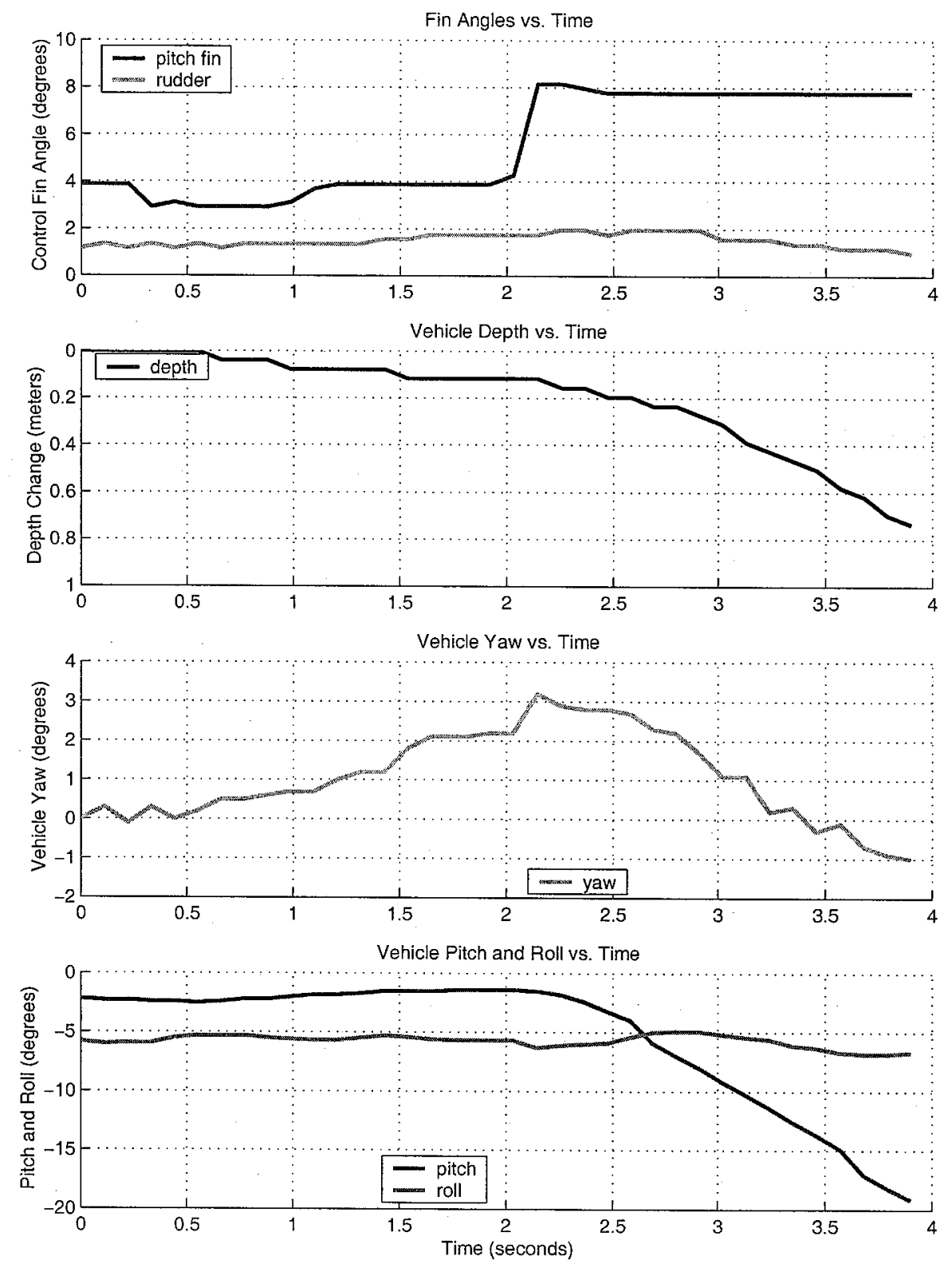

Figure 7-9: REMUS Mission Data: Vehicle pitching down. [d980729b, Obj. 22] 


\section{Chapter 8}

\section{Comparisons of Simulator Output and Experimental Data}

In this chapter, we will compare the simulator output with the vehicle response data described in Chapter 7. We discuss the discrepancies between the two data sets, and the coefficient adjustments used to correct for them.

\subsection{Model Preparation}

The model was given initial conditions and fin inputs matching the experimental data. Early model comparisons lead the author to adjust some of the vehicle coefficients.

\subsubsection{Initial Conditions}

Each run of the model was given the following initial conditions:

Table 8.1: REMUS Simulator Initial Conditions

\begin{tabular}{|c|c|c|c|}
\hline Parameter & Value & Units & Description \\
\hline$\overline{z_{g}}$ & $+1.96 e-002$ & $\mathrm{~m}$ & vertical center of gravity \\
\hline$u$ & $+1.54 \mathrm{e}+000$ & $\mathrm{~m} / \mathrm{s}$ & Forward velocity \\
\hline$\phi$ & $-5.00 e+000$ & $\operatorname{deg}$ & Roll Angle \\
\hline
\end{tabular}

The forward velocity of $1.54 \mathrm{~m} / \mathrm{s}$ ( 3 knots) is the operating speed of the vehicle at a propeller RPM of 1500 . The initial roll angle is the experimentally-measured steady-state roll offset due to propeller torque.

The remaining angles, angular rates, and velocities were entered as zero. Although vehicle rates and velocities were not measured directly in the experiments, it is assumed that they were small.

\subsubsection{Coefficient Adjustments}

The author found it necessary to adjust a subset of the vehicle coefficients derived in Chapter 4 by the factors listed below in Table 8.2.

These adjustments were based on comparisons with the experimental data, and were not entirely unexpected. The methods used in Sections 4.2 .3 and 4.3 .3 to calculate rolling resistance had a high degree of uncertainty. More accurate methods to calculate this rolling resistance and added 
Table 8.2: Vehicle Coefficient Adjustment Factors

\begin{tabular}{cll}
\hline \hline Coefficient & Adjustment Factor & Description \\
\hline$K_{p p}$ & 100 & Rolling Resistance Moment \\
$K_{\dot{p}}$ & 5 & Roll Added Mass Moment \\
$Y_{v v}$ & 10 & Sway Resistance Force \\
$Z_{w w}$ & 10 & Heave Resistance Force \\
$M_{q q}$ & 12.5 & Pitch Rate Resistance Moment \\
$N_{r r}$ & 10 & Yaw Rate Resistance Moment \\
\hline \hline
\end{tabular}

mass should be explored. Similarly, the strip integration method used in Section 4.2 .2 to estimate crossflow drag was understood to be inaccurate.

Note that Tables 4.2 and 4.3 list the unadjusted vehicle coefficients, while Appendices B and $\mathrm{C}$ list the adjusted vehicle coefficients.

\subsection{Uncertainties in Model Comparison}

The following uncertainties affected the accuracy of the model comparison:

- Vehicle Initial Conditions The greatest uncertainty was the vehicle state at the start of each experimental objective. We were unable to measure currents, wave effects, and non-axial vehicle velocities. which would have all affected the vehicle motion during open-loop maneuvers.

- Control Fin Alignment Although the alignment of the vehicle fins was checked before each experimental mission, it was difficult to keep the vehicle control fins from getting knocked during vehicle transportation and launch. This could have resulted in fin misalignments as great as five degrees.

- Attitude Sensor Dynamics The vehicle attitude sensor was sensitive to coupling due to vehicle accelerations. Although most likely a small effect, the author did not have the opportunity to characterize these sensor dynamics.

\subsection{Horizontal Plane Dynamics}

Figures 8-1 through 8-5 show the vehicle response to step changes in rudder angle. In Figures 8-1 through 8-4, the vehicle was given zero fin inputs for ten seconds, then four degrees of positive rudder for 25 seconds, then four degrees of negative rudder for 30 seconds.

The lower plot in Figure 8-2 shows a vehicle yaw rate of roughly ten degrees per second, which compares well with the experimental data in Figure 7-7, Section 7.5.1.

Figure 8-5 shows a direct comparison of the experiment and simulator data. The simulated vehicle yaw rate is shown to be a very close match to the experiment. Discrepancies between the vehicle depth rates, and vehicle pitch and roll angles likely have to do with differences in the simulator initial conditions.

\subsection{Vertical Plane Dynamics}

Unlike the horizontal plane motion, it is important to carefully consider the vehicle response to both positive and negative pitch fin angles, due to the effect of the vehicle center of gravity-center of buoyancy separation. 

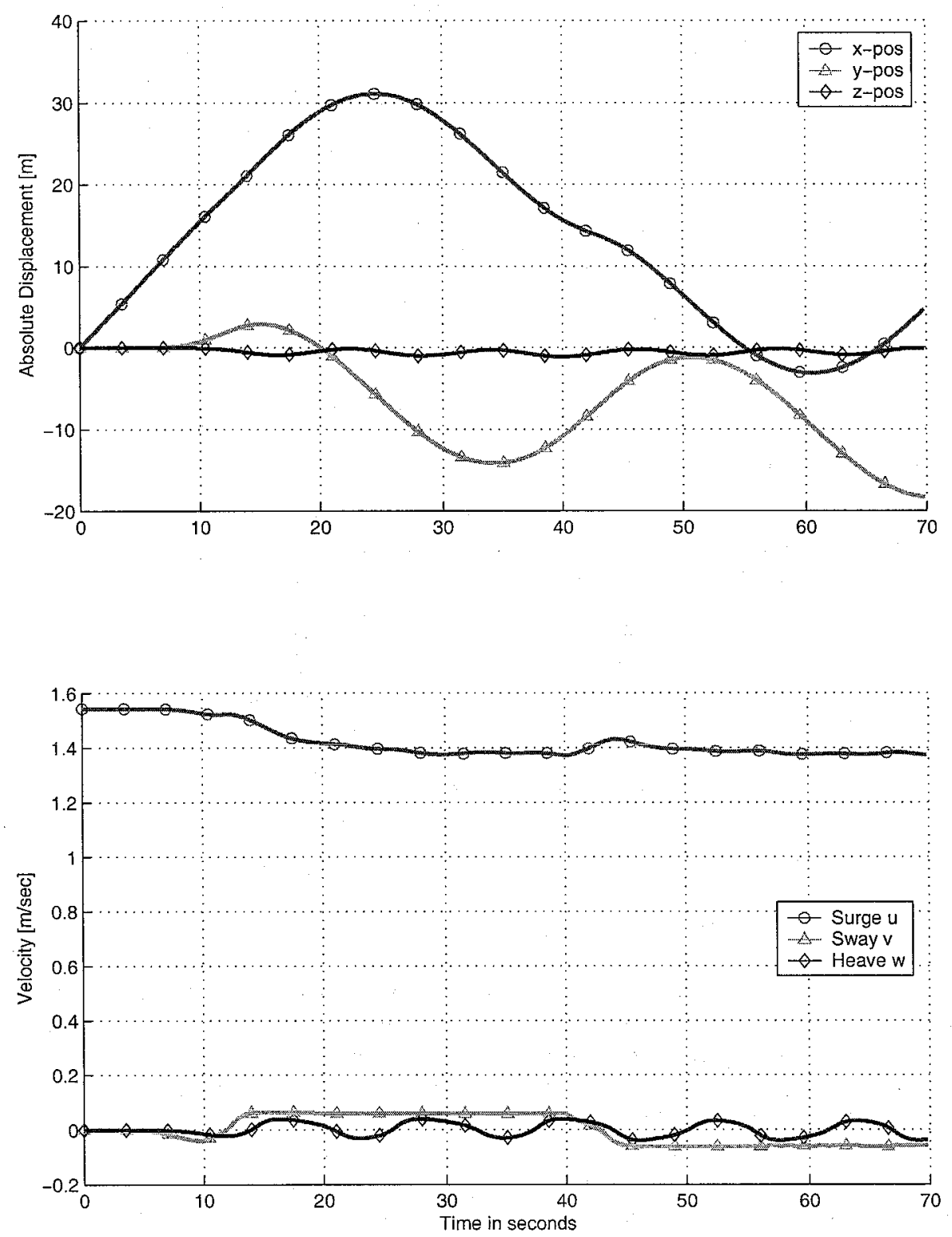

Figure 8-1: REMUS Simulator Data: Linear displacements and velocities for vehicle response in the horizontal plane 

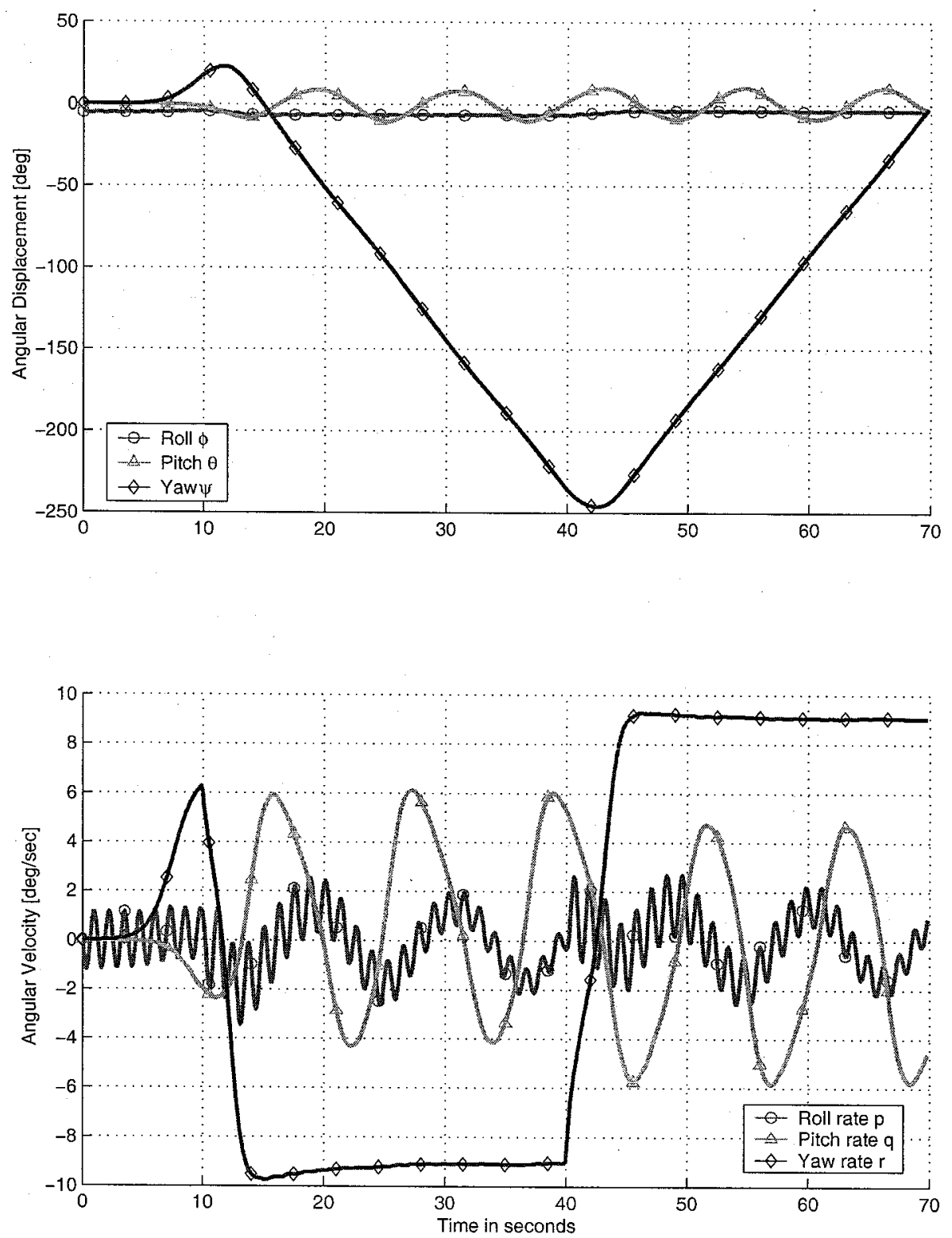

Figure 8-2: REMUS Simulator Data: Angular displacements and velocities for vehicle response in the horizontal plane 

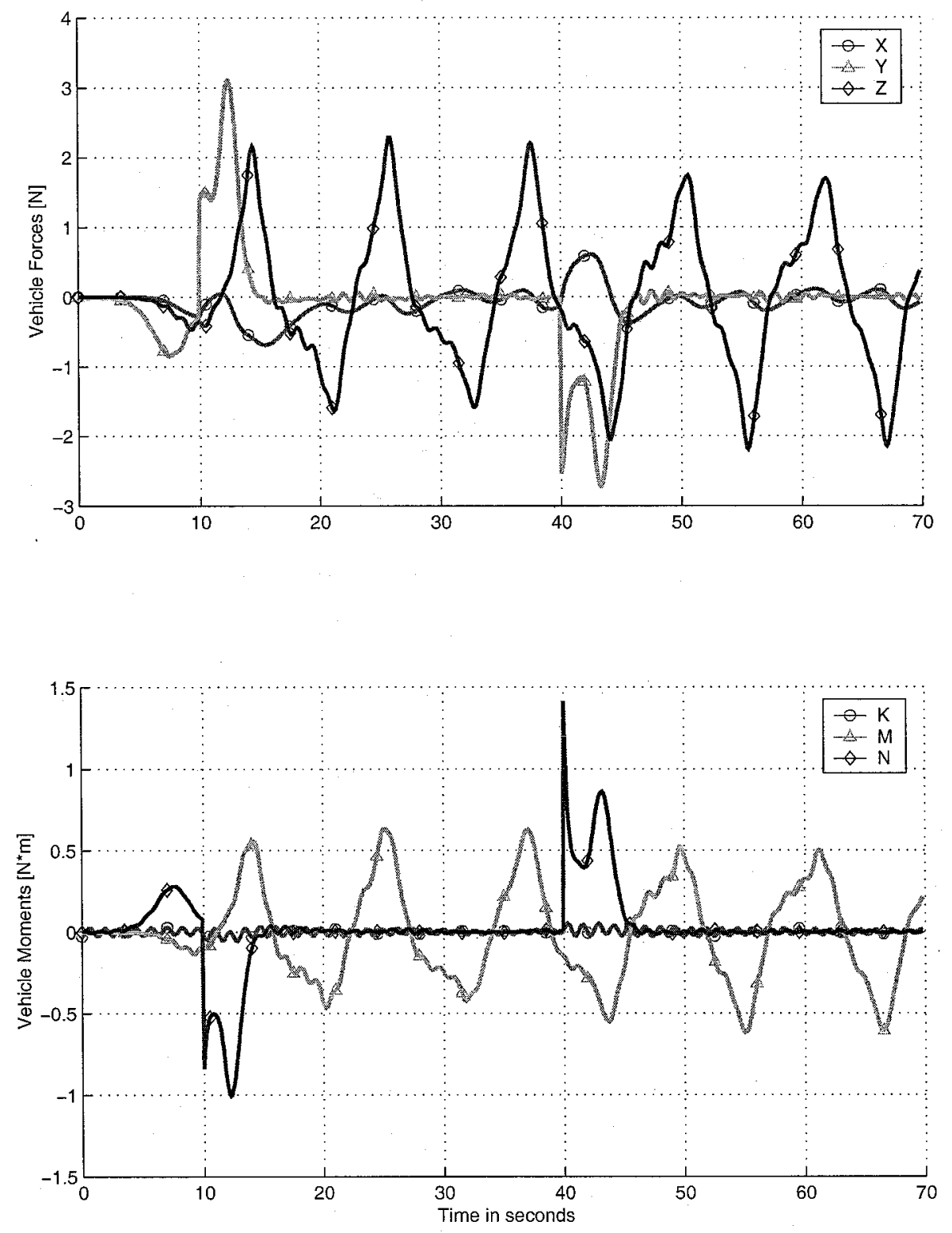

Figure 8-3: REMUS Simulator Data: Forces and moments for vehicle response in the horizontal plane 

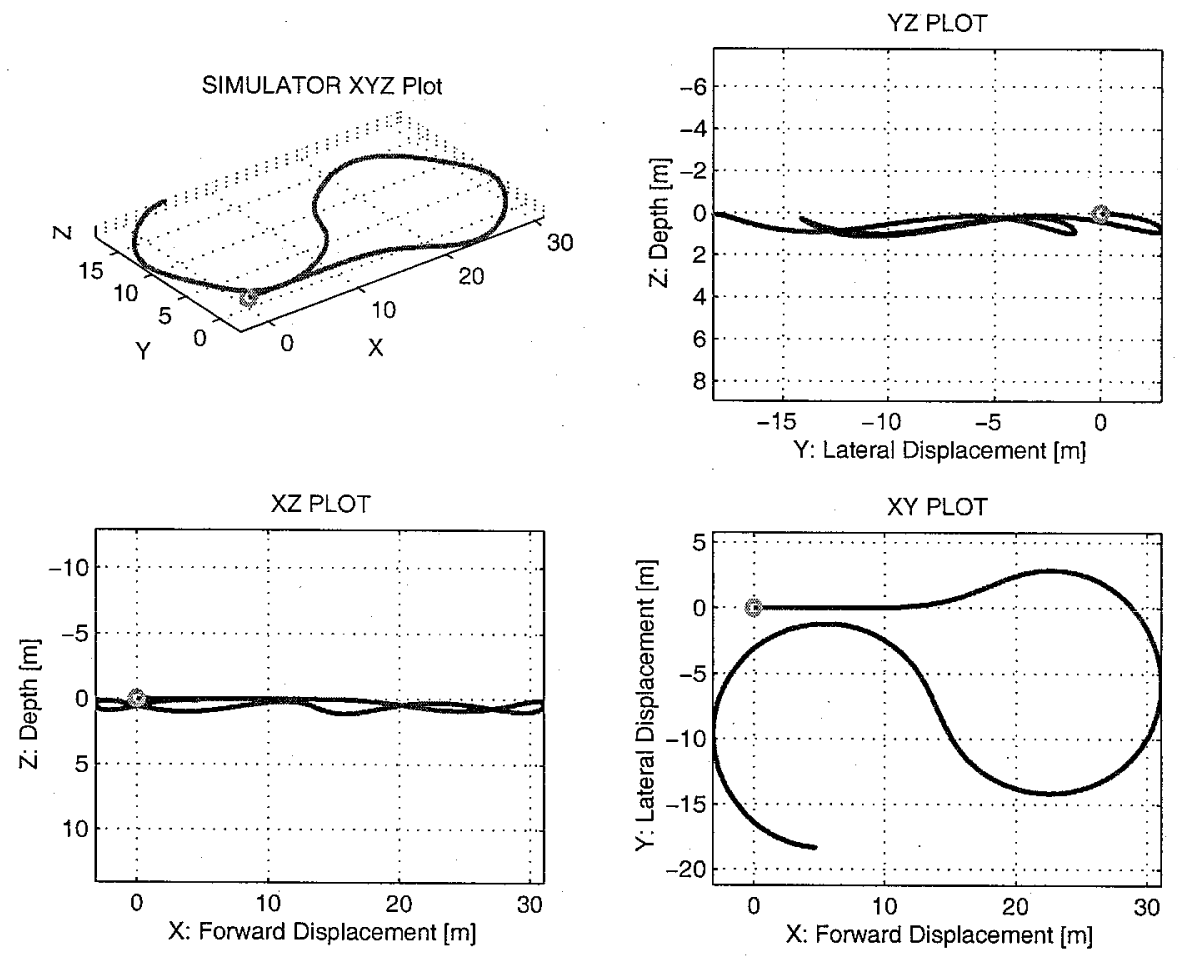

Figure 8-4: REMUS Simulator Data: Vehicle trajectory for vehicle response in the horizontal plane 

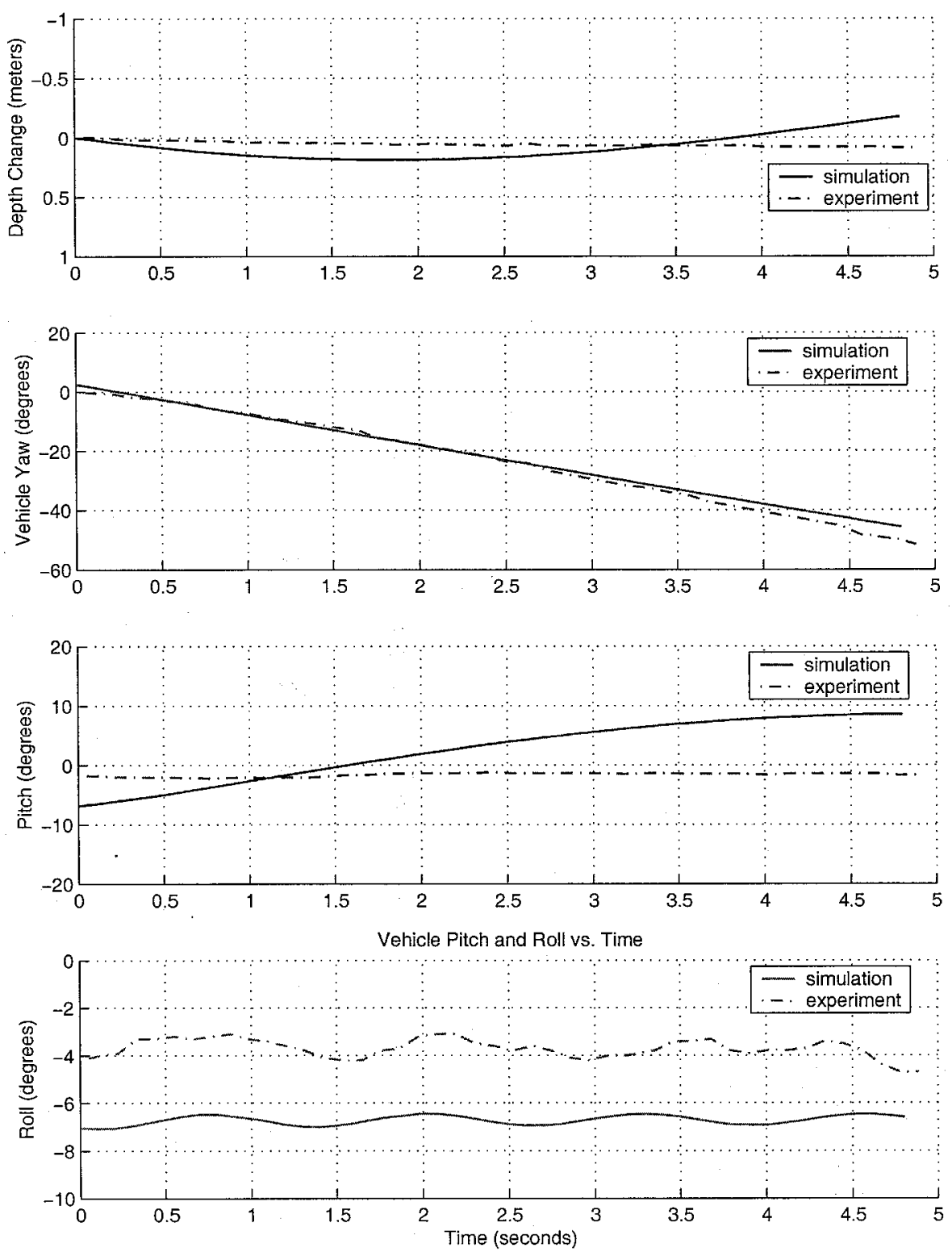

Figure 8-5: REMUS Simulator Data: Comparison plots for vehicle response in the yaw plane 


\subsubsection{Vehicle Pitching Up}

Figures 8-6 through 8-10 show the vehicle response to a step change in pitch fin angle. As shown in Figure 8-10, the vehicle was given zero fin inputs for two seconds, then four degrees of negative pitch fin for two seconds.

Figure 8-10 shows a vehicle depth change of roughly 0.5 meters and a pitch change of twenty degrees, which both compare well with the experimental data in Figure 7-8, Section 7.5.2.

\subsubsection{Vehicle Pitching Down}

Figures 8-11 through 8-15 show the vehicle response to a step change in pitch fin angle. As shown in Figure 8-15, the vehicle was given zero fin inputs for two seconds, then eight degrees of positive pitch fin for two seconds.

Figure 8-15 shows a vehicle depth change of roughly 0.6 meters and a pitch change of thirty degrees, which both compare well with the experimental data in Figure 7-9, Section 7.5.2. The model pitch rate is slightly higher than the experimental data, but this could be due a difference in initial conditions. 

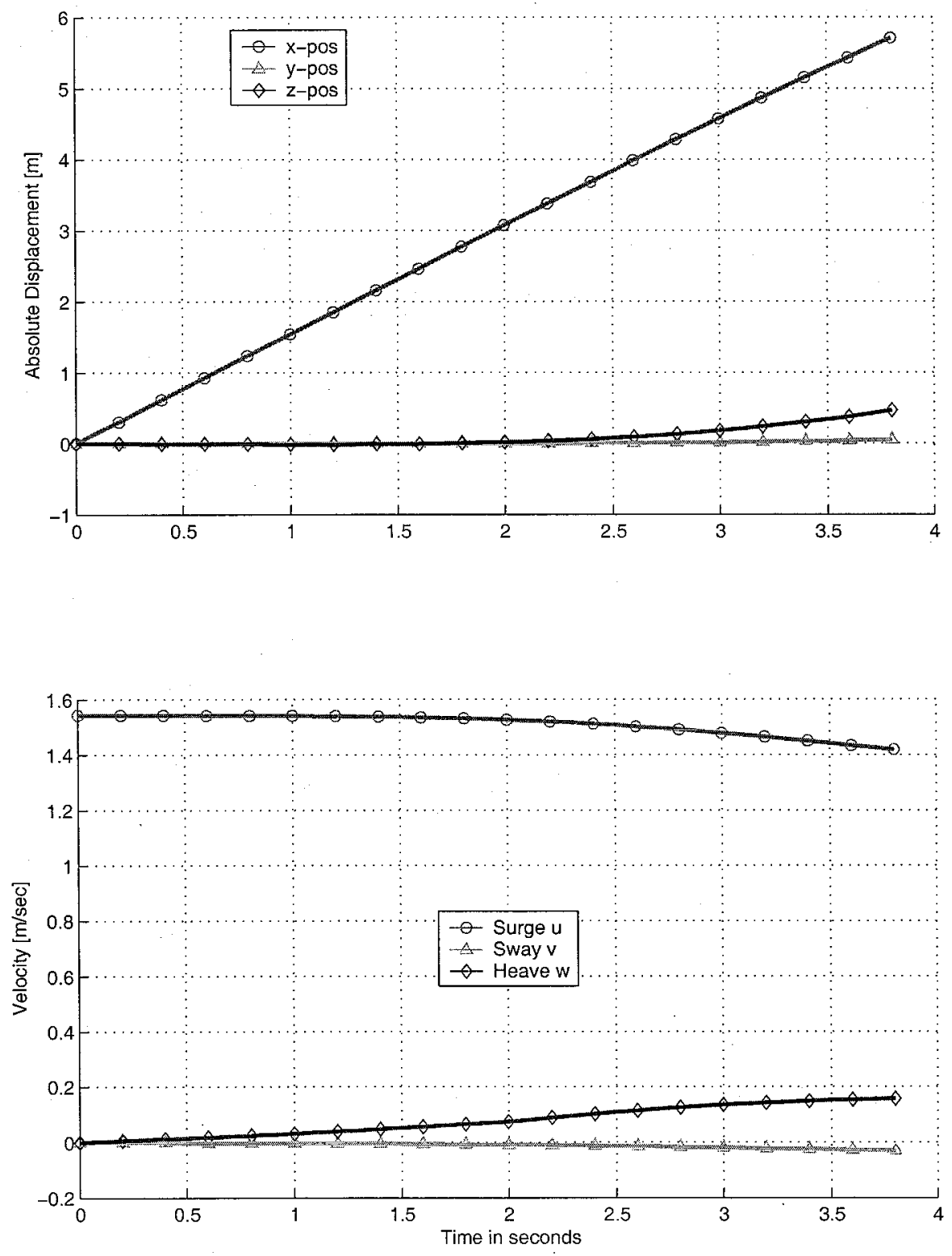

Figure 8-6: REMUS Simulator Data: Linear displacements and velocities for vehicle pitching up 

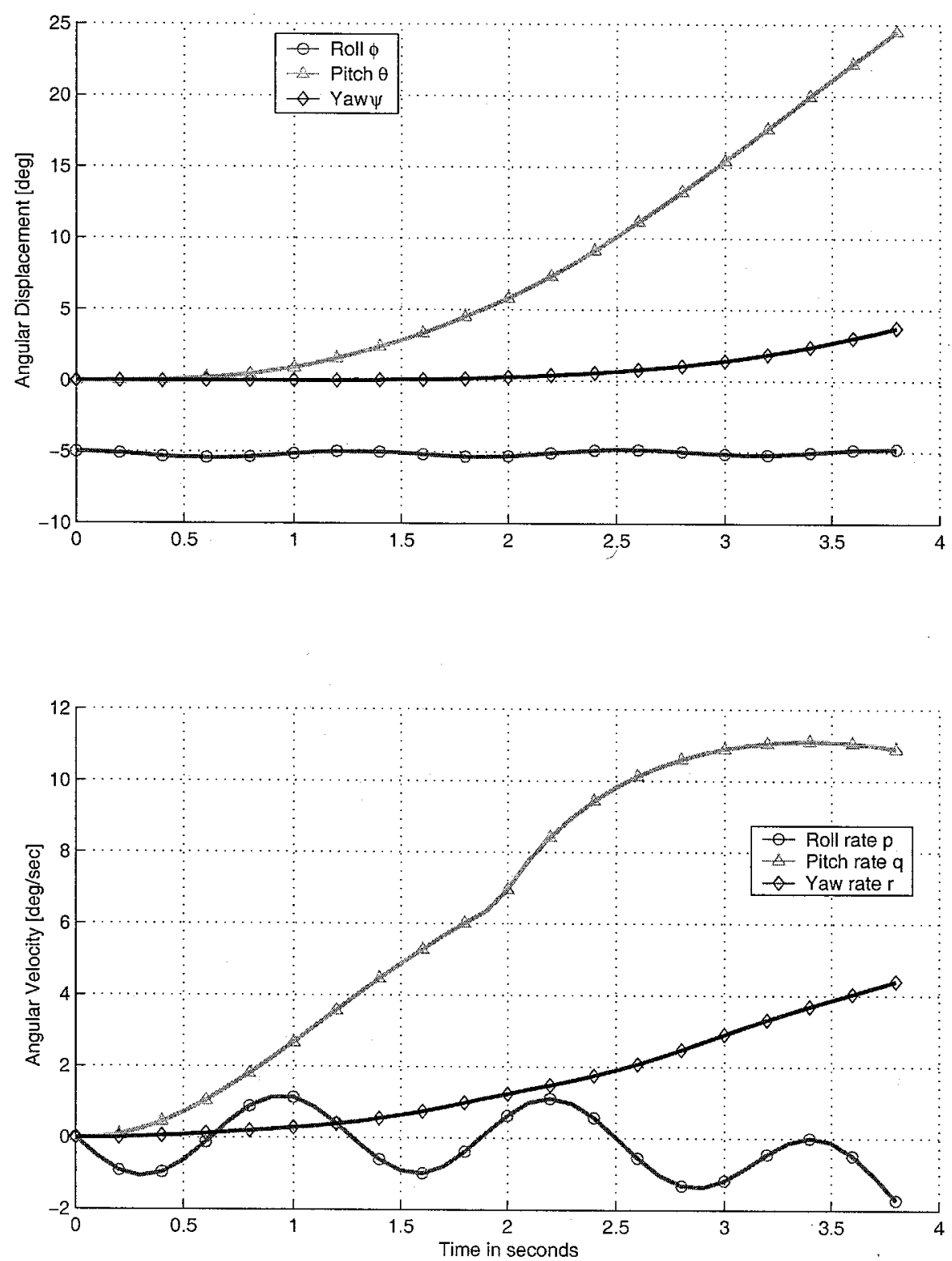

Figure 8-7: REMUS Simulator Data: Angular displacements and velocities for vehicle pitching up 

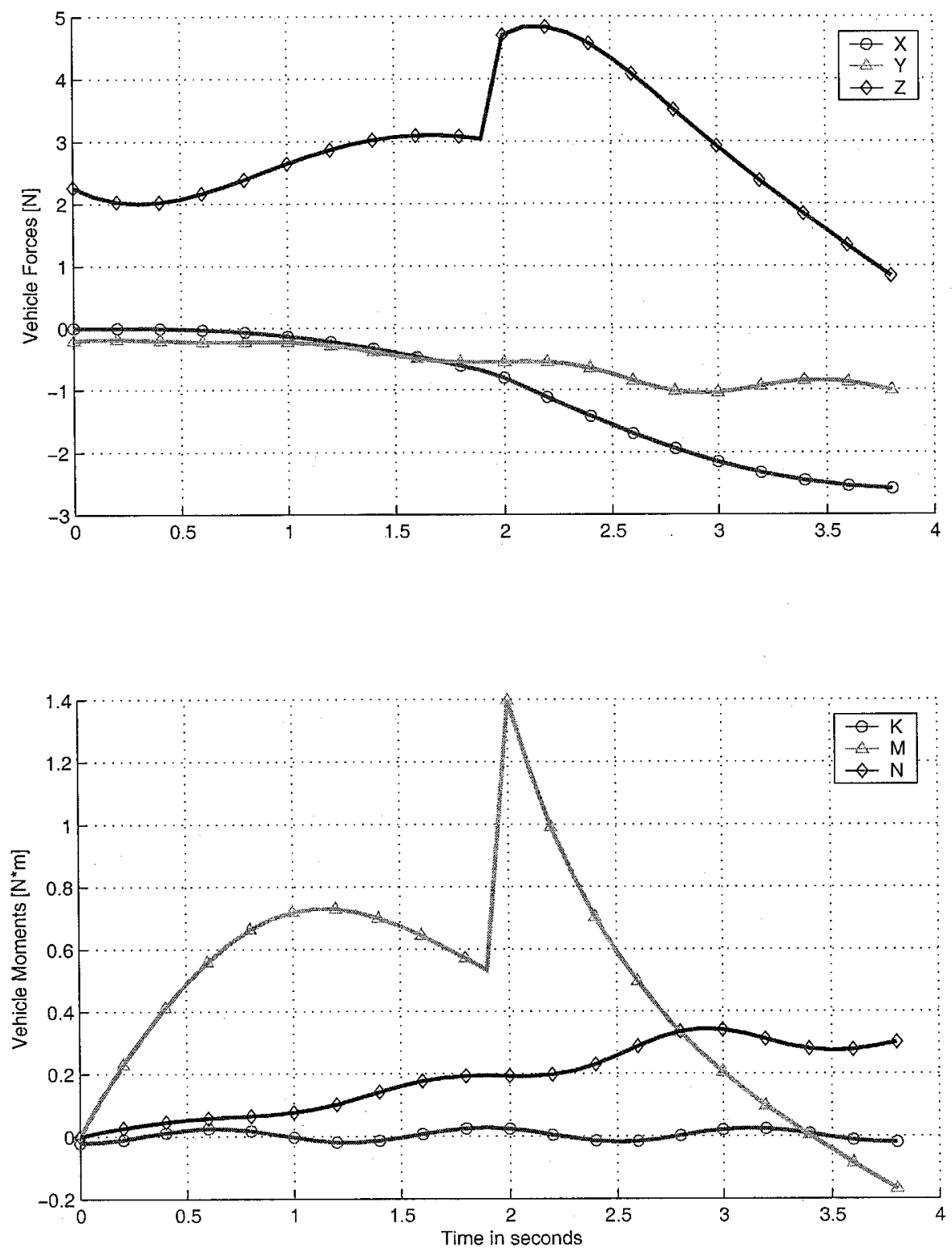

Figure 8-8: REMUS Simulator Data: Forces and moments for vehicle pitching up 

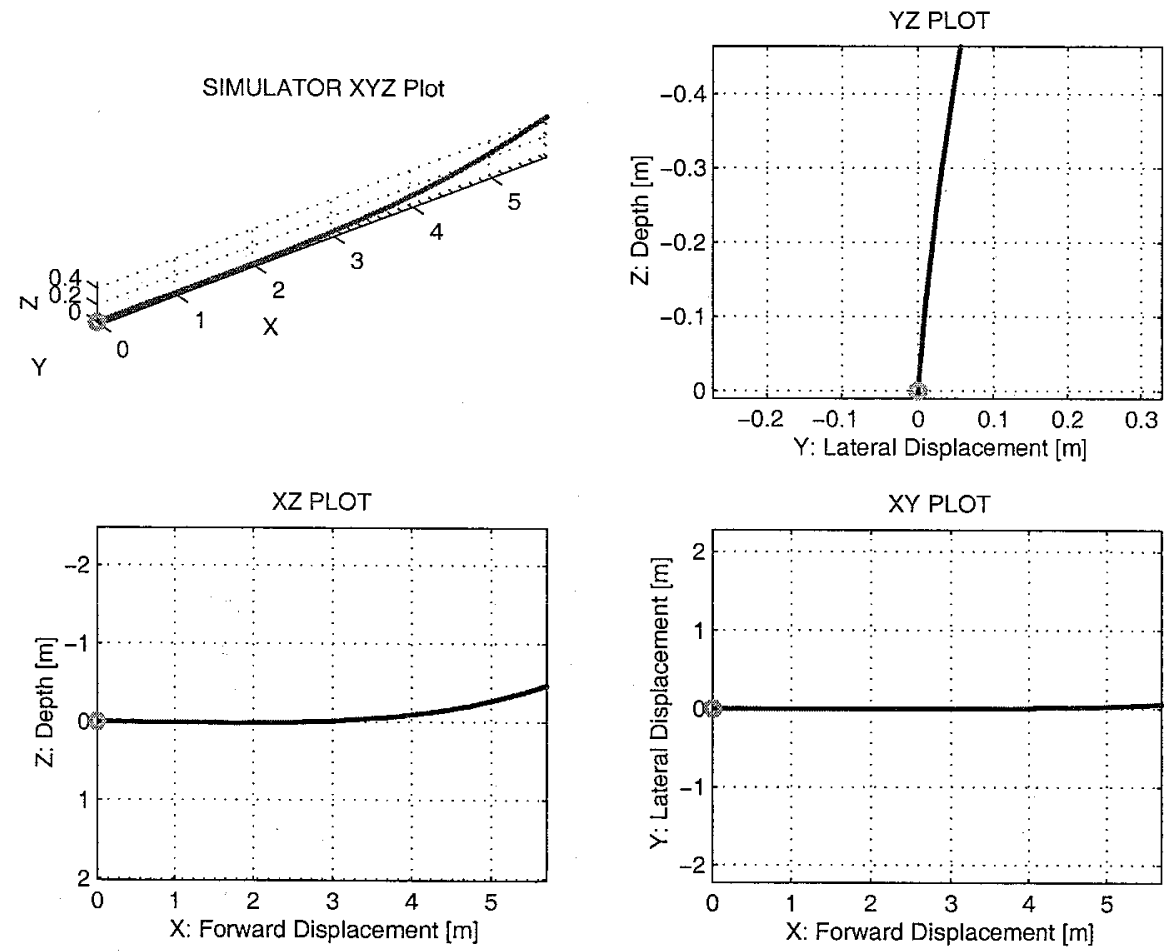

Figure 8-9: REMUS Simulator Data: Vehicle trajectory for vehicle pitching up 

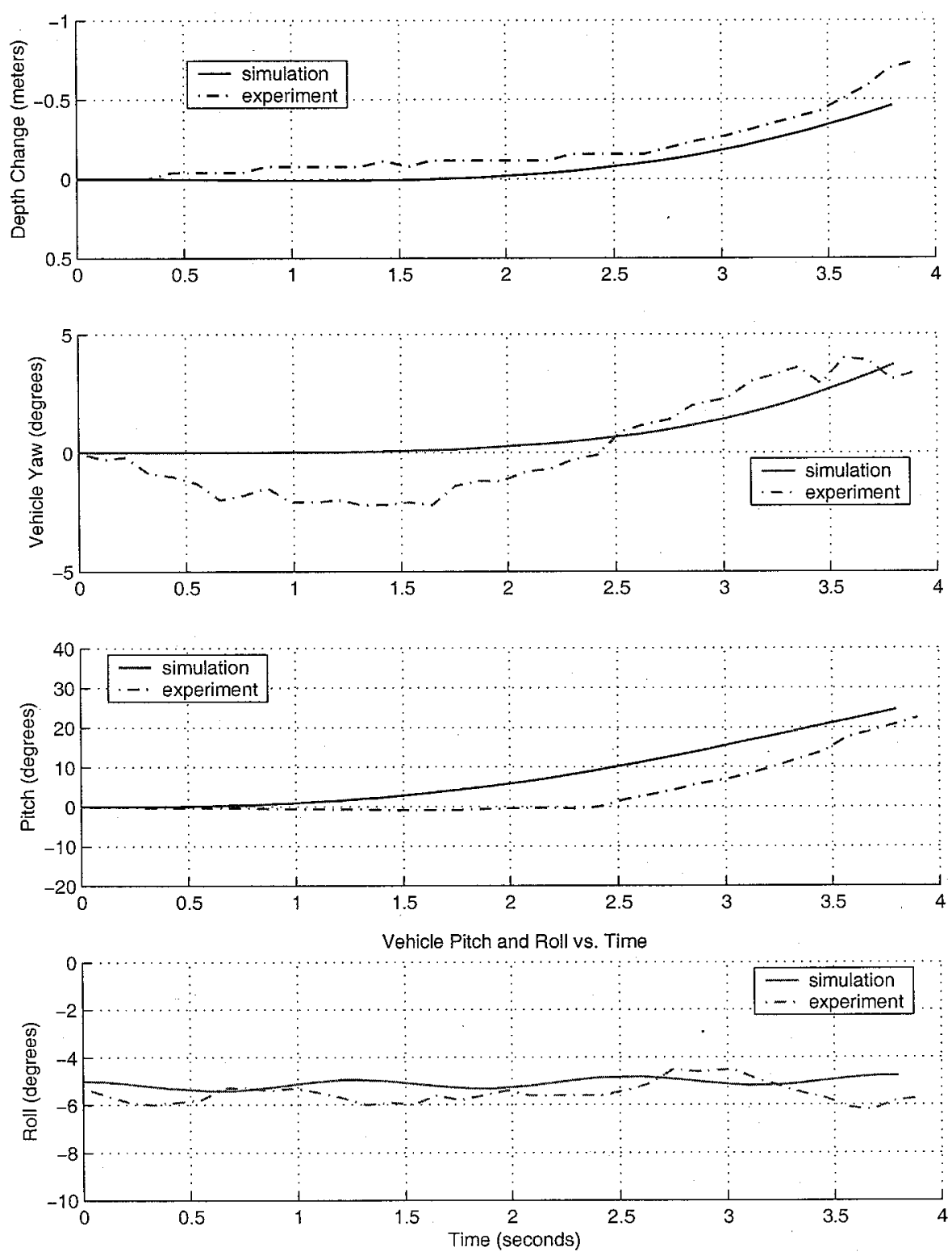

Figure 8-10: REMUS Simulator Data: Comparison plots for vehicle pitching up 

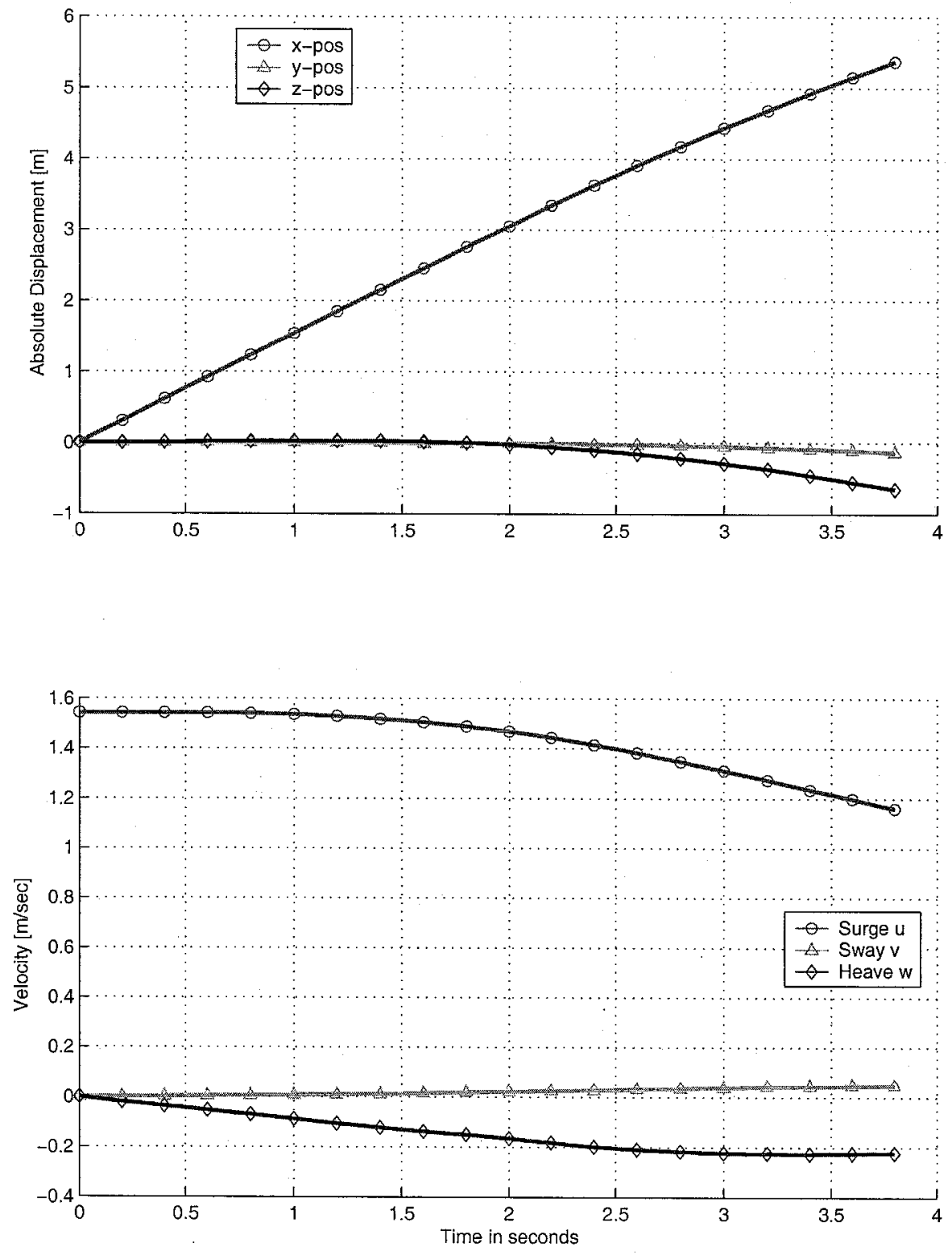

Figure 8-11: REMUS Simulator Data: Linear displacements and velocities for vehicle pitching down 

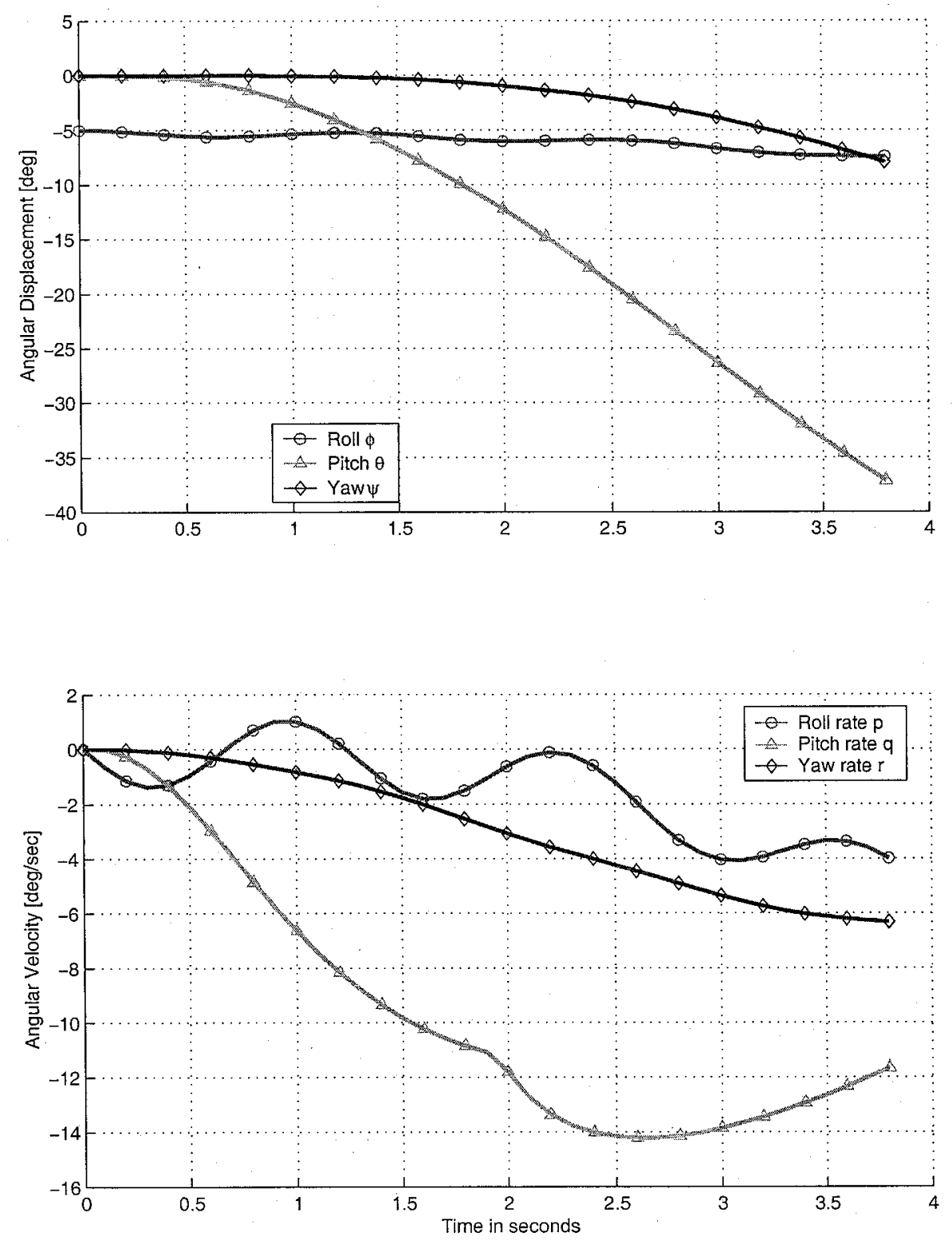

Figure 8-12: REMUS Simulator Data: Angular displacements and velocities for vehicle pitching down 

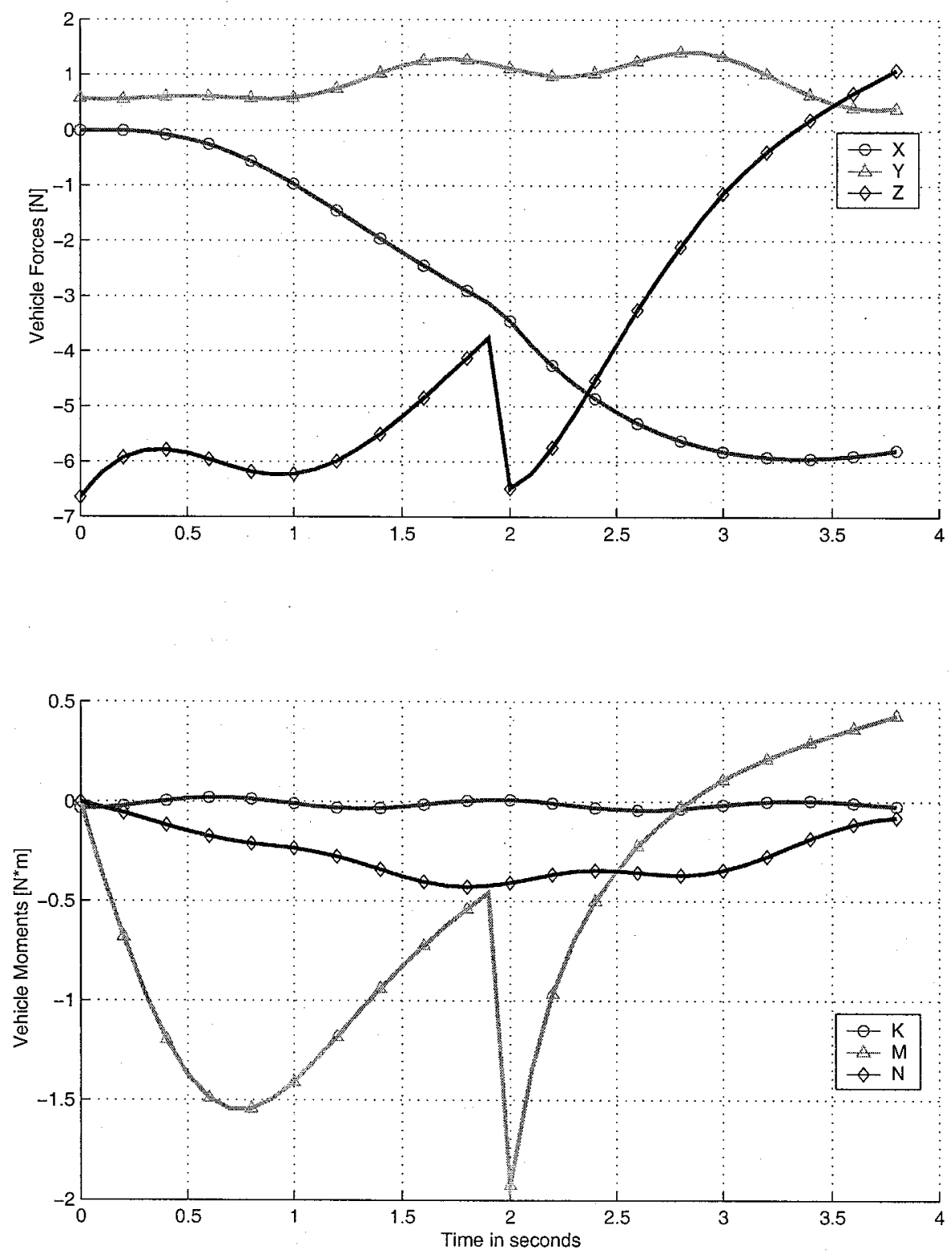

Figure 8-13: REMUS Simulator Data: Forces and moments for vehicle pitching down 

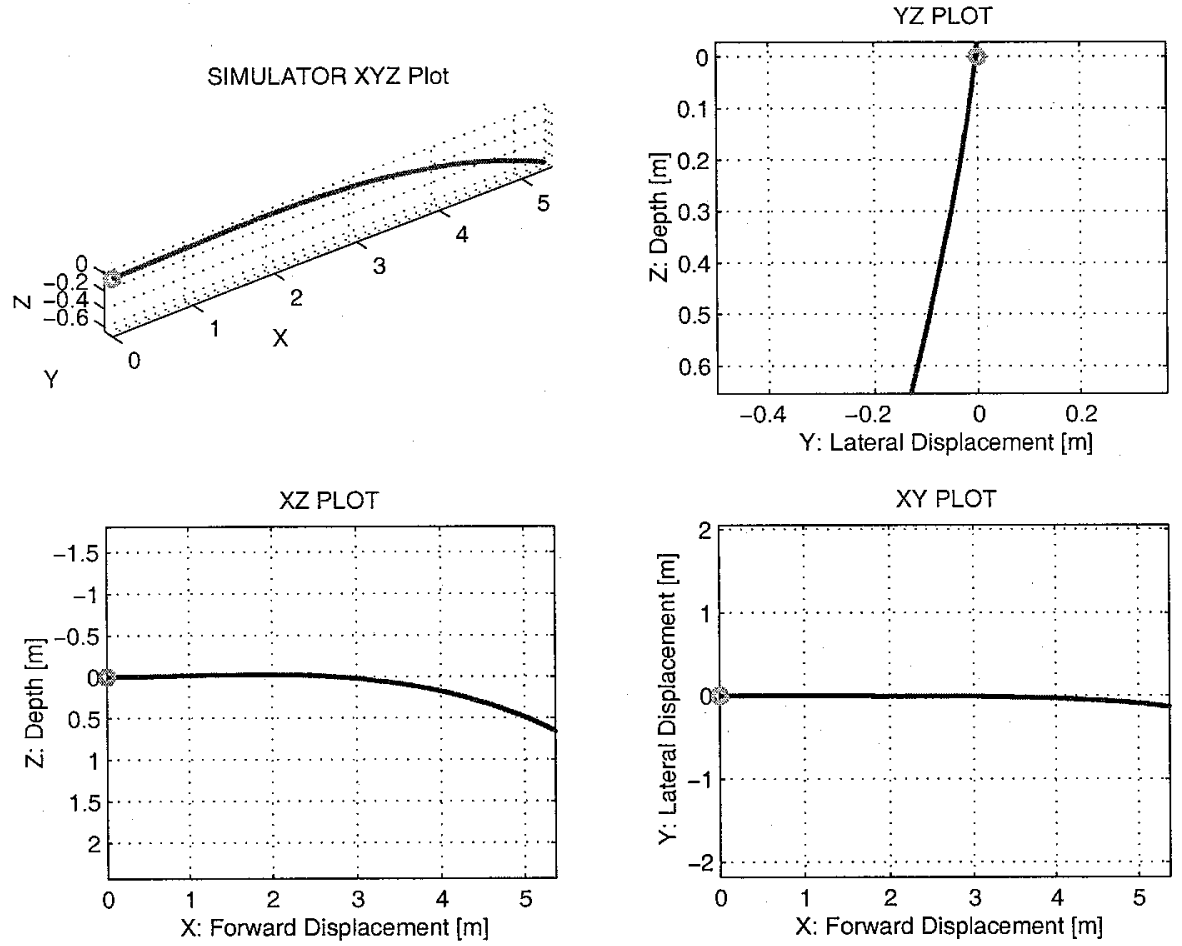

Figure 8-14: REMUS Simulator Data: Vehicle trajectory for vehicle pitching down 

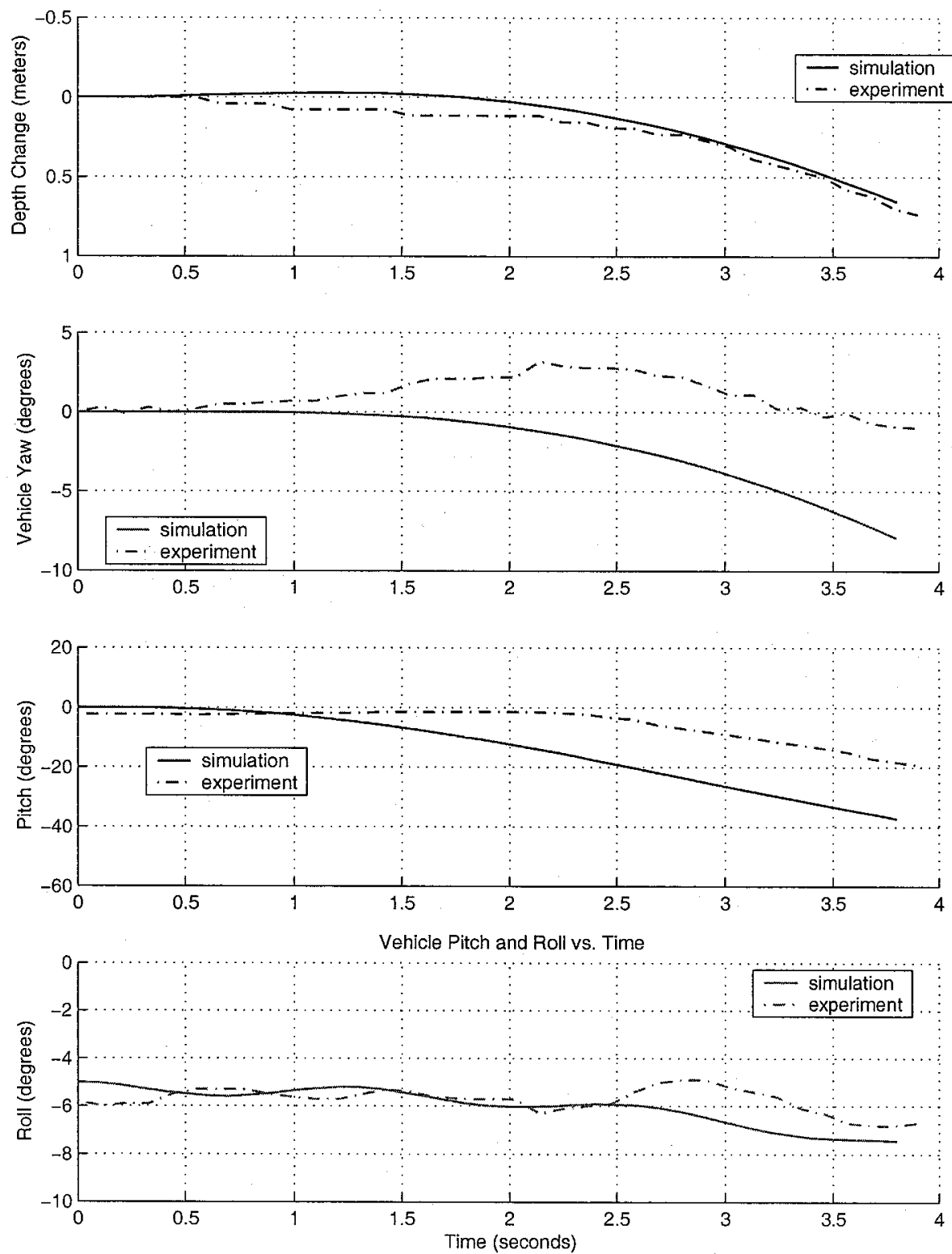

Figure 8-15: REMUS Simulator Data: Comparison plots for vehicle pitching down 


\section{Chapter 9}

\section{Linearized Depth Plane Model and Controller}

This chapter describes the depth-plane linearization of the vehicle equations of motion and the coefficients used in those equations. It demonstrates the use of that model to design a simple innerand-outer (pitch-and-depth) loop PD depth controller. Finally, this chapter shows how real world effects such as environmental disturbances, sensor noise and actuator non-linearities can be added to the model.

Although this chapter covers only the depth-plane model, the equations of motion and vehicle coefficients are explained in sufficient detail to allow the development of a more sophisticated linearized, decoupled model in five degrees of freedom (disallowing vehicle roll).

\subsection{Linearizing the Vehicle Equations of Motion}

The equations governing the motion of the vehicle are described in Chapter 3 . We will briefly describe the linearization of the vehicle kinematics, rigid-body dynamics, and mechanics.

\subsubsection{Vehicle Kinematics}

The vehicle kinematic equations are developed in Section 3.2. Note that the rotational coordinate transform matrix $J_{2}\left(\eta_{2}\right)$, described in Equation 3.5, is not defined for $\cos \theta=\frac{\pi}{2}$. This will not be a problem, given that when we linearize the model we will be assuming small vehicle perturbations about $\theta=0$.

As we are assuming pure depth-plane motion, we need only consider the body-relative surge velocity $u$, heave velocity $w$, and pitch rate $q$, and the earth-relative vehicle forward position $x$, depth $z$, and pitch angle $\theta$. We will set to zero all other velocities $(v, p, r)$, and drop the equations for any out-of-plane terms.

By these assumptions, Equation 3.1 and Equation 3.4 result in the following relationships between body- and earth-fixed vehicle velocities:

$$
\begin{aligned}
& \dot{x}=\cos \theta u+\sin \theta w \\
& \dot{z}=-\sin \theta u+\cos \theta w \\
& \dot{\theta}=q
\end{aligned}
$$

We will linearize these equations by assuming that the vehicle motion consists of small perturbations around a steady point. $U$ in this case represents the steady-state forward velocity of the vehicle; 
heave and pitch are linearized about zero.

$$
\begin{aligned}
& u=U+u^{\prime} \\
& w=w^{\prime} \\
& q=q^{\prime}
\end{aligned}
$$

We will also use the Maclaurin expansion of the trigonometric terms:

$$
\begin{aligned}
& \sin \theta=\theta-\frac{\theta^{3}}{3 !}+\frac{\theta^{5}}{5 !}+\ldots \\
& \cos \theta=1-\frac{\theta^{2}}{2 !}+\frac{\theta^{4}}{4 !}+\ldots
\end{aligned}
$$

Applying these linearizations and dropping any higher-order terms results in the following linearized kinematic relationships between earth- and body-fixed velocities:

$$
\begin{aligned}
& \dot{x}=u+\theta w \\
& \dot{z}=-U \theta+w \\
& \dot{\theta}=q
\end{aligned}
$$

\subsubsection{Vehicle Rigid-Body Dynamics}

The vehicle kinematic equations are developed in Section 3.3. As in the equations for vehicle kinematics, we will simplify the equations for the rigid body dynamics (Equation 3.8) to a description of pure depth-plane motion. We will set to zero all unrelated terms $\left(v, p, r, y_{g}\right)$, and drop the equations for out-of-plane vehicle motion:

$$
\begin{aligned}
& \sum X=m\left(\dot{u}+w q-x_{g} q^{2}+z_{g} \dot{q}\right) \\
& \sum Z=m\left(\dot{w}-u q-z_{g} q^{2}-x_{g} \dot{q}\right) \\
& \sum M=I_{y y} \dot{q}+m\left[z_{g}(\dot{u}+w q)-x_{g}(\dot{w}-u q)\right]
\end{aligned}
$$

Substituting the linearized velocities from Equation 9.2 and dropping any higher-order terms results in the following linearized equations of motion:

$$
\begin{aligned}
& \sum X=m\left[\dot{u}+z_{g} \dot{q}\right] \\
& \sum Z=m\left[\dot{w}-x_{g} \dot{q}-U q\right] \\
& \sum M=I_{y y} \dot{q}+m\left[z_{g} \dot{u}-x_{g}(\dot{w}-U q)\right]
\end{aligned}
$$

\subsubsection{Vehicle Mechanics}

Our assumptions about the vehicle mechanics are identical to those developed in Section 3.4. In the linearized vehicle equations of motion, external forces and moments

$$
\sum F_{\text {ext }}=F_{\text {hydrostatic }}+F_{\text {lift }}+F_{\text {drag }}+F_{\text {control }}+F_{\text {disturbance }}
$$

are described in terms of vehicle coefficients. For example, linearized axial drag

$$
F_{\mathrm{d}}=-\left(\frac{1}{2} \rho c_{d} A_{f} U\right) u=X_{u} u \quad \Longrightarrow \quad X_{u}=\frac{\partial F_{\mathrm{d}}}{\partial u}=-\frac{1}{2} \rho c_{d} A_{f} U
$$


These linearized coefficients are based on a combination of theoretical equations and empiricallyderived formulae. Note that we neglect the forward force due to body lift as it is a nonlinear term.

\subsection{Linearized Coefficient Derivation}

The various parameters necessary to derive the vehicle coefficients are either included in the section describing the coefficient, or are listed in the Appendix.

\subsubsection{Hydrostatics}

The nonlinear equations for hydrostatic forces and moments (see Equation 4.3) are developed in Section 4.1. We will simplify these by dropping the out-of-plane terms, assuming that $x_{g} \approx x_{b}$, and using the Maclaurin expansion of the trigonometric terms (see Equation 9.3). We then drop the higher-order terms, as well as any resulting constant terms. This yields the following linearized hydrostatic equations:

$$
\begin{aligned}
& X_{\theta}=-(W-B) \theta \\
& M_{\theta}=-\left(z_{g} W-z_{b} B\right) \theta
\end{aligned}
$$

\subsubsection{Axial Drag}

Vehicle axial drag can be expressed in Equation 4.6. Linearizing this equation using the relationship given in Equation 9.2, results in the following:

$$
\begin{aligned}
& X=-\frac{1}{2} \rho c_{d} A_{f}(U+u)|U+u| \\
& X=-\frac{1}{2} \rho c_{d} A_{f}\left(U^{2}+2 U u+u^{2}\right)
\end{aligned}
$$

Assuming

$$
u \ll U, \quad U>0
$$

and dropping any constant terms results in the following linearized axial drag coefficient:

$$
X_{u}=-\rho c_{d} A_{f} U
$$

\subsubsection{Crossflow Drag}

Vehicle crossflow drag is discussed in Section 4.2.2. In order to linearize the quadratic crossflow drag coefficients described in Equation 4.8, we must linearize the heave and pitch perturbation velocities about zero. This is accomplished by fitting a slope to the parabolic velocity-squared curve, as show in Figure 9-1.

$$
\begin{aligned}
w^{2} & \approx m_{\mathrm{w}} w \\
q^{2} & \approx m_{\mathrm{q}} q
\end{aligned}
$$

The actual values used in this parameterization are given in Table 9.1. The estimates for maximum expected heave and pitch velocity were taken from field data. 


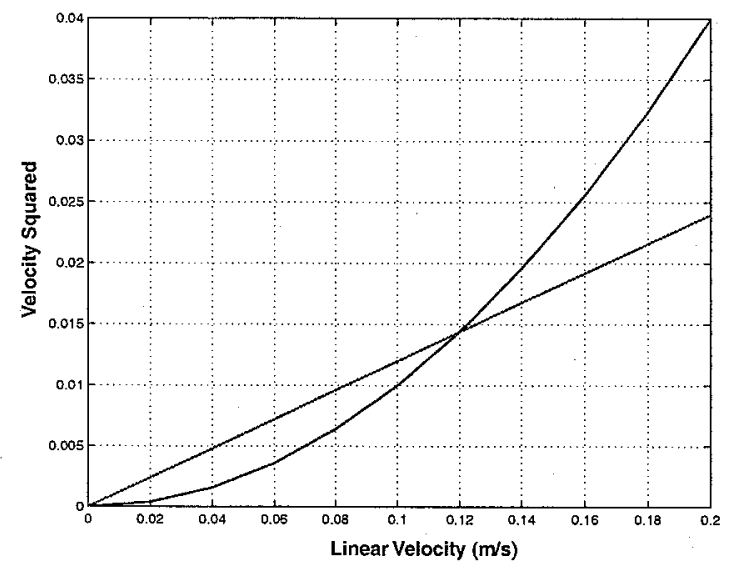

Figure 9-1: Perturbation Velocity Linearization

Table 9.1: Linearized Velocity Parameters

\begin{tabular}{cccl}
\hline \hline Parameter & Value & Units & Description \\
\hline$w_{\max }$ & $+2.00 \mathrm{e}-001$ & $\mathrm{~m} / \mathrm{s}$ & Maximum Heave Perturbation \\
$q_{\max }$ & $+5.00 \mathrm{e}-001$ & $\mathrm{rad} / \mathrm{s}$ & Maximum Pitch Perturbation \\
$m_{\mathrm{w}}$ & $+1.20 \mathrm{e}-001$ & $\mathrm{~m} / \mathrm{s}$ & Heave Coefficient \\
$m_{\mathrm{q}}$ & $+3.00 \mathrm{e}-001$ & $\mathrm{rad} / \mathrm{s}$ & Pitch Coefficient \\
\hline \hline
\end{tabular}

Substituting these parameters results in the following linear equations for crossflow drag:

$$
\begin{aligned}
Z_{w c} & =-\frac{1}{2} \rho c_{\mathrm{dc}} m_{w} \int_{x_{t}}^{x_{b 2}} 2 R(x) \mathrm{d} x-2 \cdot\left(\frac{1}{2} \rho S_{\mathrm{fin}} c_{\mathrm{df}} m_{w}\right) \\
M_{w c} & =-\frac{1}{2} \rho c_{\mathrm{dc}} m_{w} \int_{x_{t}}^{x_{b 2}} 2 x R(x) \mathrm{d} x-2 x_{\mathrm{fin}} \cdot\left(\frac{1}{2} \rho S_{\mathrm{fin}} c_{\mathrm{df}} m_{w}\right) \\
Z_{q c} & =-\frac{1}{2} \rho c_{\mathrm{dc}} m_{q} \int_{x_{t}}^{x_{b 2}} 2 x|x| R(x) \mathrm{d} x-2 x_{\mathrm{fin}}\left|x_{\mathrm{fin}}\right| \cdot\left(\frac{1}{2} \rho S_{\mathrm{fin}} c_{\mathrm{df}} m_{q}\right) \\
M_{q c} & =-\frac{1}{2} \rho c_{\mathrm{dc}} m_{q} \int_{x_{t}}^{x_{b 2}} 2 x^{3} R(x) \mathrm{d} x-2 x_{\mathrm{fin}}^{3} \cdot\left(\frac{1}{2} \rho S_{\mathrm{fin}} c_{\mathrm{df}} m_{q}\right)
\end{aligned}
$$

See Table A.2 for the limits of integration.

\subsubsection{Added Mass}

Vehicle added mass is discussed in Section 4.3. By substituting linearized velocities and dropping the out-of-plane and higher-order terms, the non-linear equations (see Equation 4.15) reduce to the following:

$$
\begin{aligned}
X_{\mathrm{A}} & =X_{\dot{u}} \dot{u}+Z_{\dot{q}} m_{\mathrm{q}} q \\
Z_{\mathrm{A}} & =Z_{\dot{w}} \dot{w}+Z_{\dot{q}} \dot{q}-X_{\dot{u}} U q \\
M_{\mathrm{A}} & =M_{\dot{w}} \dot{w}+M_{\dot{q}} \dot{q}-\left(Z_{\dot{w}}-X_{\dot{u}}\right) U w-Z_{\dot{q}} U q
\end{aligned}
$$


Linearized axial added mass is still given by the equation:

$$
X_{\dot{u}}=-m_{11}
$$

and crossflow added masses are given by the equations:

$$
\begin{aligned}
Z_{\dot{w}}= & -\int_{x_{\mathrm{t}}}^{x_{\mathrm{f}}} m_{22}(x) \mathrm{d} x-\int_{x_{\mathrm{f}}}^{x_{\mathrm{f} 2}} m_{22 f}(x) \mathrm{d} x-\int_{x_{\mathrm{f} 2}}^{x_{\mathrm{b} 2}} m_{22}(x) \mathrm{d} x \\
M_{\dot{w}}= & Z_{\dot{q}}= \\
& -\int_{x_{\mathrm{t}}}^{x_{\mathrm{f}}} x m_{22}(x) \mathrm{d} x-\int_{x_{\mathfrak{f}}}^{x_{\mathrm{f} 2}} x m_{22 f}(x) \mathrm{d} x-\int_{x_{\mathrm{f} 2}}^{x_{\mathrm{b} 2}} x m_{22}(x) \mathrm{d} x \\
M_{\dot{q}}= & -\int_{x_{\mathrm{t}}}^{x_{\mathrm{f}}} x^{2} m_{22}(x) \mathrm{d} x-\int_{x_{\mathrm{f}}}^{x_{\mathrm{f} 2}} x^{2} m_{22 f}(x) \mathrm{d} x-\int_{x_{\mathrm{f} 2}}^{x_{\mathrm{b}}} x^{2} m_{22}(x) \mathrm{d} x .
\end{aligned}
$$

See Table A.2 for the limits of integration.

The remaining cross-terms result from added mass coupling:

$$
\begin{aligned}
X_{q a} & =Z_{\dot{q}} m_{\mathrm{q}} \\
Z_{q a} & =-X_{\dot{u}} U \\
M_{w a} & =-\left(Z_{\dot{u}}-X_{\dot{u}}\right) U \\
M_{q a} & =-Z_{\dot{q}} U
\end{aligned}
$$

The added mass cross-term $M_{w A}$ is also called the Munk Moment, and relates to the pure moment experienced by a body in ideal, inviscid flow at an angle of attack.

\subsubsection{Body Lift Force and Moment}

The non-linear equations for vehicle body lift are discussed in Section 4.4. Linearizing the vehicle velocities according to Equation 9.2 and substituting into Equation 4.27 the relationships given above, we are left with the following, linearized equations for vehicle body lift:

$$
Z_{L}=-\frac{1}{2} \rho d^{2} c_{y d \beta} U w
$$

which results in the body lift coefficient :

$$
Z_{w l}=-\frac{1}{2} \rho d^{2} c_{y d \beta} U
$$

and body lift moment:

$$
M_{u l l}=-\frac{1}{2} \rho d^{2} c_{y d \beta} x_{c p} U
$$

\subsubsection{Fin Lift}

Vehicle fin lift is discussed in Section 4.5

Dropping the out-of-plane terms in the equations for effective fin velocities (see Equation 4.40) leads to the following:

$$
\begin{aligned}
& u_{\text {fin }}=u \\
& w_{\text {fin }}=w-x_{\text {fin }} q
\end{aligned}
$$


Effective fin angle $\delta_{e}$ can be expressed as

$$
\delta_{e}=\delta_{s}+\beta_{e}
$$

where $\delta_{s}$ is the stern plane angle, and $\beta_{e}$ the effective angle of attack of the fin zero plane, as shown in Figure 4-2. This effective angle is expressed as

$$
\beta_{e}=\frac{w_{\mathrm{fin}}}{u_{\mathrm{fin}}} \approx \frac{1}{u}\left(w-x_{\mathrm{fin}} q\right)
$$

After linearizing the velocities in Equations 4.44 and 4.45 according to Equation 9.2 results in the following sets of fin lift coefficients:

$$
\begin{aligned}
& Z_{\delta_{s}}=-\rho c_{L \alpha} S_{\mathrm{fin}} U^{2} \\
& Z_{w f}=-\frac{1}{2} \rho c_{L \alpha} S_{\mathrm{fin}} U \\
& Z_{q f}=\frac{1}{2} \rho c_{L \alpha} S_{\mathrm{fin}} x_{\mathrm{fin}} U
\end{aligned}
$$

and fin moment coefficients:

$$
\begin{aligned}
& M_{\delta_{s}}=\rho c_{L \alpha} S_{\mathrm{fin}} x_{\mathrm{fin}} U^{2} \\
& M_{w f}=\frac{1}{2} \rho c_{L \alpha} S_{\mathrm{fin}} x_{\mathrm{fin}} U \\
& M_{q f}=-\frac{1}{2} \rho c_{L \alpha} S_{\mathrm{fin}} x_{\mathrm{fin}}^{2} U
\end{aligned}
$$

\subsubsection{Combined Terms}

The sum of the depth-plane forces and moments on the vehicle can be expressed as:

$$
\begin{aligned}
& \sum X=X_{\dot{u}} \dot{u}+X_{u} u+X_{q} q+X_{\theta} \theta \\
& \sum Z=Z_{\dot{w}} \dot{w}+Z_{\dot{q}} \dot{q}+Z_{w} w+Z_{q} q+Z_{\delta_{s}} \delta_{s} \\
& \sum M=M_{\dot{w}} \dot{w}+M_{\dot{q}} \dot{q}+M_{w} w+M_{q} q+M_{\theta} \theta+M_{\delta_{s}} \delta_{s}
\end{aligned}
$$

where

$$
\begin{aligned}
Z_{w} & =Z_{w c}+Z_{w l}+Z_{w f} \\
M_{w} & =M_{w c}+M_{w a}+M_{w l}+M_{w f} \\
Z_{q} & =Z_{q c}+Z_{q a}+Z_{q f} \\
M_{q} & =M_{q c}+M_{q a}+M_{q f}
\end{aligned}
$$

The values for these combined terms are given in Table 9.2 .

\subsubsection{Linearized Coefficients}

The final values for the linearized coefficients are given below in Table 9.3. 
Table 9.2: Combined Linearized Coefficients

\begin{tabular}{cccl}
\hline \hline Parameter & Value & Units & Description \\
\hline$Z_{w c}$ & $-1.57 \mathrm{e}+001$ & $\mathrm{~kg} / \mathrm{s}$ & Crossflow Drag \\
$Z_{w l}$ & $-3.45 \mathrm{e}+001$ & $\mathrm{~kg} \cdot \mathrm{m} / \mathrm{s}$ & Body Lift \\
$Z_{w f}$ & $-1.64 \mathrm{e}+001$ & $\mathrm{~kg} \cdot \mathrm{m} / \mathrm{s}$ & Fin Lift \\
& & & \\
$Z_{q c}$ & $+1.20 \mathrm{e}-001$ & $\mathrm{~kg} \cdot \mathrm{m} / \mathrm{s}$ & Crossflow Drag \\
$Z_{q a}$ & $+1.44 \mathrm{e}+000$ & $\mathrm{~kg} \cdot \mathrm{m} / \mathrm{s}$ & Added Mass Cross Term \\
$Z_{q f}$ & $-1.12 \mathrm{e}+001$ & $\mathrm{~kg} \cdot \mathrm{m} / \mathrm{s}$ & Fin Lift \\
& & & \\
$M_{w c}$ & $-4.03 e-001$ & $\mathrm{~kg} \cdot \mathrm{m} / \mathrm{s}$ & Crossflow Drag \\
$M_{w a}$ & $+5.34 \mathrm{e}+001$ & $\mathrm{~kg} \cdot \mathrm{m} / \mathrm{s}$ & Added Mass Cross Term \\
$M_{w l}$ & $-1.11 \mathrm{e}+001$ & $\mathrm{~kg} \cdot \mathrm{m} / \mathrm{s}$ & Body Lift \\
$M_{w f}$ & $-1.12 \mathrm{e}+001$ & $\mathrm{~kg} \cdot \mathrm{m} / \mathrm{s}$ & Fin Lift \\
& & & \\
$M_{q c}$ & $-2.16 \mathrm{e}+000$ & $\mathrm{~kg} \cdot \mathrm{m}^{2} / \mathrm{s}$ & Crossflow Drag \\
$M_{q a}$ & $+2.97 \mathrm{e}+000$ & $\mathrm{~kg} \cdot \mathrm{m}^{2} / \mathrm{s}$ & Added Mass Cross Term \\
$M_{q f}$ & $-7.68 e+000$ & $\mathrm{~kg} \cdot \mathrm{m}^{2} / \mathrm{s}$ & Fin Lift \\
\hline \hline
\end{tabular}

\subsection{Linearized Equations of Motion}

We will now combine the equations developed in the preceding chapters to develop the linearized equations of motion.

\subsubsection{Equations of Motion}

Combining Equations 9.6 and 9.25 results in the following linearized vehicle equations of motion:

$$
\begin{gathered}
\left(m-X_{\dot{u}}\right) \dot{u}+m z_{g} \dot{q}-X_{u} u-X_{q} q-X_{\theta} \theta=0 \\
\left(m-Z_{\dot{w}}\right) \dot{w}-\left(m x_{g}+Z_{\dot{q}}\right) \dot{q}-Z_{w} w-\left(m U+Z_{q}\right) q=Z_{\delta_{s}} \delta_{s} \\
m z_{g} \dot{u}-\left(m x_{g}+M_{\dot{w}}\right) \dot{w}+\left(I_{y y}-M_{\dot{q}}\right) \dot{q} \\
\quad-M_{w} w+\left(m x_{g} U-M_{q}\right) q-M_{\theta} \theta=M_{\delta_{s}} \delta_{s}
\end{gathered}
$$

if we assume $z_{g}$ is small compared to the other terms, we can decouple heave and pitch from surge, resulting in the following equations of motion:

$$
\begin{aligned}
& \left(m-Z_{\dot{w}}\right) \dot{w}-\left(m x_{g}+Z_{\dot{q}}\right) \dot{q}-Z_{w} w-\left(m U+Z_{q}\right) q=Z_{\delta_{s}} \delta_{s} \\
& -\left(m x_{g}+M_{\dot{w}}\right) \dot{w}+\left(I_{y y}-M_{\dot{q}}\right) \dot{q} \\
& \quad-M_{w} w+\left(m x_{g} U-M_{q}\right) q-M_{\theta} \theta=M_{\delta_{s}} \delta_{s}
\end{aligned}
$$

Similarly, the kinematic equations of motion from Equation 9.4 reduce to:

$$
\begin{aligned}
& \dot{z}=w-\dot{U} \theta \\
& \dot{\theta}=q
\end{aligned}
$$


Table 9.3: Linearized Maneuvering Coefficients

\begin{tabular}{cccl}
\hline \hline Parameter & Value & Units & Description \\
\hline$X_{\theta}$ & $+8.90 \mathrm{e}+000$ & $\mathrm{~kg} \cdot \mathrm{m} / \mathrm{s}^{2}$ & Hydrostatic \\
$X_{u}$ & $-1.35 \mathrm{e}+001$ & $\mathrm{~kg} / \mathrm{s}$ & Axial Drag \\
$X_{\dot{u}}$ & $-9.30 \mathrm{e}-001$ & $\mathrm{~kg}$ & Added Mass \\
$X_{q}$ & $-5.78 \mathrm{e}-001$ & $\mathrm{~kg} \cdot \mathrm{m} / \mathrm{s}$ & Added Mass Cross Term \\
& & & \\
$Z_{w}$ & $-6.66 \mathrm{e}+001$ & $\mathrm{~kg} / \mathrm{s}$ & Combined Term \\
$Z_{q}$ & $-9.67 \mathrm{e}+000$ & $\mathrm{~kg} \cdot \mathrm{m} / \mathrm{s}$ & Combined Term \\
$Z_{\dot{w}}$ & $-3.55 \mathrm{e}+001$ & $\mathrm{~kg}$ & Added Mass \\
$Z_{\dot{q}}$ & $-1.93 \mathrm{e}+000$ & $\mathrm{~kg} \cdot \mathrm{m}$ & Added Mass \\
$Z_{\delta_{s}}$ & $-5.06 \mathrm{e}+001$ & $\mathrm{~kg} \cdot \mathrm{m} / \mathrm{s}^{2}$ & Fin Lift \\
& & & \\
$M_{\theta}$ & $-5.77 \mathrm{e}+000$ & $\mathrm{~kg} \cdot \mathrm{m}^{2} / \mathrm{s}^{2}$ & Hydrostatic \\
$M_{w}$ & $+3.07 \mathrm{e}+001$ & $\mathrm{~kg} \cdot \mathrm{m} / \mathrm{s}$ & Combined Term \\
$M_{q}$ & $-6.87 \mathrm{e}+000$ & $\mathrm{~kg} \cdot \mathrm{m}^{2} / \mathrm{s}$ & Combined Term \\
$M_{\dot{w}}$ & $-1.93 \mathrm{e}+000$ & $\mathrm{~kg} \cdot \mathrm{m}$ & Added Mass \\
$M_{\dot{q}}$ & $-4.88 \mathrm{e}+000$ & $\mathrm{~kg} \cdot \mathrm{m}^{2}$ & Added Mass \\
$Z_{\delta_{s}}$ & $-3.46 \mathrm{e}+001$ & $\mathrm{~kg} \cdot \mathrm{m}^{2} / \mathrm{s}^{2}$ & Fin Lift \\
\hline \hline
\end{tabular}

\subsubsection{Four-term State Vector}

We will find it convenient to combine Equations 9.28 and 9.29 into a single equation in matrix form, as follows:

$$
\begin{gathered}
{\left[\begin{array}{cccc}
m-X_{\dot{u}} & -\left(m x_{s}+Z_{\dot{q}}\right) & 0 & 0 \\
-\left(m x_{g}+M_{\dot{w}}\right) & I_{y y}-M_{\dot{q}} & 0 & 0 \\
0 & 0 & 1 & 0 \\
0 & 0 & 0 & 1
\end{array}\right]\left[\begin{array}{c}
\dot{w} \\
\dot{q} \\
\dot{z} \\
\dot{\theta}
\end{array}\right]} \\
- \\
-\left[\begin{array}{cccc}
Z_{w} & m U+Z_{q} & 0 & 0 \\
M_{w} & -m x_{g} U+M_{q} & 0 & M_{\theta} \\
1 & 0 & 0 & -U \\
0 & 1 & 0 & 0
\end{array}\right]\left[\begin{array}{c}
w \\
q \\
z \\
\theta
\end{array}\right]=\left[\begin{array}{c}
Z_{\delta_{s}} \\
M_{\delta_{s}} \\
0 \\
0
\end{array}\right]\left[\delta_{s}\right]
\end{gathered}
$$

Given the state vector

$$
x=\left[\begin{array}{llll}
w & q & z & \theta
\end{array}\right]^{T}
$$

and the input vector

$$
u=\left[\delta_{s}\right]^{T}
$$

we can write Equation 9.30 as

$$
M \dot{\boldsymbol{x}}-C_{d} x=D u
$$

or

$$
\dot{x}=M^{-1} C_{d} x+M^{-1} D u
$$

which is typically represented using the notation

$$
\dot{x}=A x+B u
$$

Substituting the coefficient values developed in Chapter 4 and listed in Appendix D, we arrive 
at the following matrices:

$$
\begin{aligned}
& A=M^{-1} C_{d}=\left[\begin{array}{llll}
-2.38 & +1.26 & +0.00 & +0.04 \\
+4.23 & -1.12 & +0.00 & -0.70 \\
+1.00 & +0.00 & +0.00 & -1.54 \\
+0.00 & +1.00 & +0.00 & +0.00
\end{array}\right] \\
& B=M^{-1} D=\left[\begin{array}{l}
-1.38 \\
-3.84 \\
+0.00 \\
+0.00
\end{array}\right]
\end{aligned}
$$

\subsubsection{Three-term State Vector}

Assuming that the heave velocities are small compared to the other terms, we can further reduce the equations of motion to the following:

$$
\left[\begin{array}{ccc}
I_{y y}-M_{\dot{q}} & 0 & 0 \\
0 & 1 & 0 \\
0 & 0 & 1
\end{array}\right]\left[\begin{array}{c}
\dot{q} \\
\dot{z} \\
\dot{\theta}
\end{array}\right]
$$

$$
+\left[\begin{array}{ccc}
-M_{q} & 0 & -M_{\theta} \\
0 & 0 & U \\
-1 & 0 & 0
\end{array}\right]\left[\begin{array}{c}
q \\
z \\
\theta
\end{array}\right]=\left[\begin{array}{c}
M_{\delta_{s}} \\
0 \\
0
\end{array}\right]\left[\delta_{s}\right]
$$

which we can simply to :

$$
\left[\begin{array}{c}
\dot{q} \\
\dot{z} \\
\dot{\theta}
\end{array}\right]=\left[\begin{array}{ccc}
\frac{M_{q}}{I_{y y}-M_{\dot{q}}} & 0 & \frac{M_{\theta}}{I_{y y}-M_{\dot{q}}} \\
0 & 0 & -U \\
1 & 0 & 0
\end{array}\right]\left[\begin{array}{c}
q \\
z \\
\theta
\end{array}\right]+\left[\begin{array}{c}
\frac{M_{\delta_{s}}}{I_{y y}-M_{\dot{q}}} \\
0 \\
0
\end{array}\right]\left[\delta_{s}\right]
$$

Again substituting the coefficient values developed in Chapter 4 and listed in Appendix D, and applying the form:

$$
\dot{x}=A x+B u
$$

we arrive at the following matrices:

$$
\begin{aligned}
& \boldsymbol{A}=\left[\begin{array}{lll}
-0.82 & +0.00 & -0.69 \\
+0.00 & +0.00 & -1.54 \\
+1.00 & +0.00 & +0.00
\end{array}\right] \\
& \boldsymbol{B}=\left[\begin{array}{l}
-4.16 \\
+0.00 \\
+0.00
\end{array}\right]
\end{aligned}
$$

\subsection{Control System Design}

We will now look at the design of a simple vehicle controller, using the state equations developed for the three-term state vector model (see Equation 9.38). The example controller, which is similar to the actual vehicle controller, consists of an inner proportional and derivative (PD) pitch loop, and an outer proportional depth loop. We will address the design of each of these controllers in turn. 


\subsubsection{Vehicle Transfer Functions}

The first step in designing the vehicle control system is to derive the vehicle transfer functions. First, we want to derive the transfer function for the inner pitch loop, relating input stern plane angle $\delta_{s}$ to the output vehicle pitch angle $\theta$. By taking the Laplace transform of Equation 9.38 , we can express this open-loop transfer function as:

$$
G_{\theta}(s)=\frac{\theta(s)}{\delta_{s}(s)}=\frac{\frac{M_{\delta_{s}}}{I_{y y}-M_{\dot{q}}}}{s^{2}-\frac{M_{q}}{I_{y y}-M_{\dot{q}}} s-\frac{M_{\theta}}{I_{y y}-M_{\dot{q}}}}
$$

Next we want to find the transfer function of the outer depth loop, which relates the input vehicle pitch angle $\theta_{d}$ to the output vehicle depth $z$. In actuality, the inner pitch loop responds sufficiently fast enough compared to the outer depth loop that we can consider the desired vehicle pitch $\theta_{d}$ to be the same as the actual vehicle pitch $\theta$. Again taking the Laplace transform of the vehicle state equations, Equation 9.38, we arrive at the desired open-loop transfer function:

$$
G_{z}(s)=\frac{z(s)}{\theta(s)}=-\frac{U}{s}
$$

\subsubsection{Control Law}

We will now define the control law for the inner and outer loops. As stated in at the beginning of this chapter, we will design a proportional-derivative (PD) inner loop, and a proportional outer loop. The control law, then, for the inner loop can be expressed as:

$$
\frac{\delta_{s}(s)}{e_{\theta}(s)}=-K_{p}\left(\tau_{d} s+1\right)
$$

where

$$
e_{\theta}=\theta_{d}-\theta
$$

$K_{p}$ is the proportional gain, and $\tau_{d}$ the derivative time constant in seconds.

There is a minus sign applied to the proportional gain due to the difference in sign conventions between the stern plane angle and vehicle pitch angle. Positive stern plane angle will generate a negative moment about the y-axis, forcing the vehicle to pitch down (negative pitch rate).

The control law for the outer loop can be expressed as:

$$
\frac{\theta(s)}{e_{z}(s)}=\gamma
$$

where

$$
e_{z}=z_{d}-z
$$

and $\gamma$ is the proportional gain.

We can express the vehicle control system as a block diagram:

\subsubsection{Controller Design Procedure}

Before getting into the specifics of selecting the vehicle controller gains, we will first review a general controller design procedure.

This procedure assumes that the system has a second order response. Given that we have chosen to work with the three-term state vector, this is true for the inner pitch loop. This assumption is also valid for higher-order systems, provided the higher order poles are at least five times further from the origin of the s-plane than the two dominant poles. 


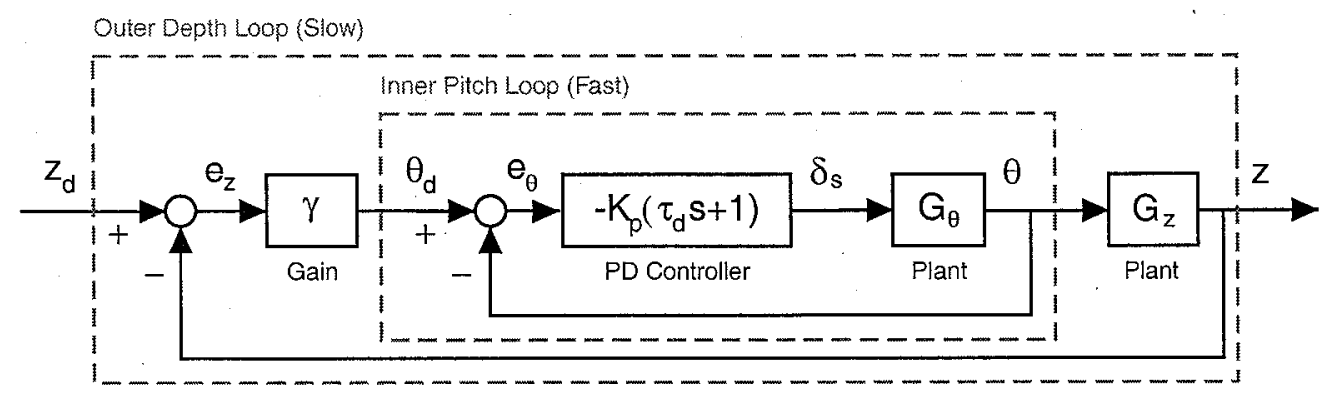

Figure 9-2: Depth-Plane Control System Block Diagram

We will design our controller to have a specific second-order response in terms of natural frequency $\omega_{n}$ and damping ratio $\zeta$, where, for a second order system:

$$
G(s)=\frac{K}{s^{2}+2 \zeta \omega_{n} s+\omega_{n}^{2}}
$$

\section{Percent Overshoot and Settling or Peak Time}

Our primary consideration in choosing a system response will be the percentage overshoot, \%OS, and peak or settling time, $T_{p}$ or $T_{s}$. See Nise [25] for a graphical explanation of these terms.

Our desired damping ratio is a function of percent overshoot, and is given by the equation:

$$
\zeta=\frac{-\ln \left(\frac{\% \mathrm{OS}}{100}\right)}{\left[\pi^{2}+\ln ^{2}\left(\frac{\% \mathrm{OS}}{100}\right)\right]^{\frac{1}{2}}}
$$

Table 9.4 gives a range of values. One can also plot lines of constant damping ratio on the pole-zero diagram.

Table 9.4: Percent Overshoot and Damping Ratio

\begin{tabular}{|c|c|c|c|c|c|c|}
\hline$\%$ OS & 5 & 10 & 15 & 20 & 25 & 30 \\
\hline$\zeta$ & 0.690 & 0.591 & 0.517 & 0.456 & 0.404 & 0.358 \\
\hline
\end{tabular}

Given the desired damping ratio, our desired natural frequency can be found through the equation:

$$
\omega_{n}=\frac{\pi}{T_{p} \sqrt{1-\zeta^{2}}}
$$

or

$$
\omega_{n}=\frac{4}{\zeta T_{s}}
$$

\section{Desired Poles}

From Equation 9.47, the second order transfer function, we can now find the desired pole locations through the quadratic formula:

$$
s_{1,2}=-\zeta \omega_{n} \pm \frac{1}{2} \sqrt{4 \zeta^{2} \omega_{n}^{2}-4 \omega_{n}^{2}}
$$




\subsubsection{Pitch Loop Controller Gains}

Substituting the coefficient values from Table 9.3 into Equation 9.41 results in the following openloop transfer function:

$$
G_{\theta}(s)=\frac{-3.18}{s^{2}+1.09 s+0.52}
$$

and the following open-loop poles.

$$
s_{1,2}=-0.55 \pm 0.47 i
$$

Figure 9-3 plots these open-loop poles. Figure 9-4 shows the open-loop step response.

Given that the REMUS vehicle operates in shallow water and cannot tolerate significant depth overshoot, I chose the following parameters in designing the vehicle pitch controller:

$$
\begin{aligned}
\% \mathrm{OS} & =0.05 \\
T_{p} & =0.75 \text { seconds }
\end{aligned}
$$

These values resulted in the damping ratio $\zeta$ and natural frequency $\omega_{n}$, shown in the legend of the root-locus plot on Figure 9-5.

There are many systematic methods for generating the necessary controller gains--in this case, I set the derivative time constant $\tau_{d}$, and used the root-locus plot to find the necessary gain $K_{p}$. The resulting controller values are also shown in the legend of Figure 9-5.

The resulting closed loop response of the pitch loop transfer function plus controller, $H_{\theta}$, is shown in Figure 9-6.

\subsubsection{Depth Loop Controller Gains}

The method used to find the depth loop controller gains is similar to that of the previous section. The depth transfer function $G_{z}$ adds a third pole, as shown in the pole-zero plot in Figure 9-7.

In order to ensure that the pitch loop response is sufficiently faster than the depth loop response, we must ensure that the pitch loop poles end up at least five times further away from the origin than the depth loop pole.

This was accomplished using a root-locus plot to identify the appropriate proportional gain $\gamma$. The resulting value can be found in the legend of the root-locus plot shown in Figure 9-8. The resulting closed loop response can be seen in Figure 9-9.

\subsection{Real-World Phenomena}

Although this controller appears to exhibit ideal performance characteristics, it is not of much use in the real world. For example, if the controller was given a sufficiently large depth error, there would be nothing to prevent it from commanding preposterous pitch and stern plane angles of greater than 180 degrees. In other words, as designed, the controller assumes the transfer function relationships to be linear out to infinity. In reality, the stern planes will stall at an effective fin angle of greater than about 12 degrees. Similarly, the REMUS vehicle shuts down at greater than 30 degrees of pitch angle, as the software assumes that something has gone disastrously wrong with the vehicle mission.

Furthermore, the simulation assumes that the vehicle sensors are free from noise, and the vehicle experiences no unmodeled disturbances. In reality, this is rarely the case.

It is an interesting exercise to discretize the transfer functions, and attempt to incorporate some of these real-world effects into the vehicle model. Figure 9-10 shows a block diagram which incorporates the saturation of the commanded stern plane and vehicle pitch angles, as well as pitch sensor noise and environmental disturbances. Figures 9-11 and 9-14 show the results of this kind of 


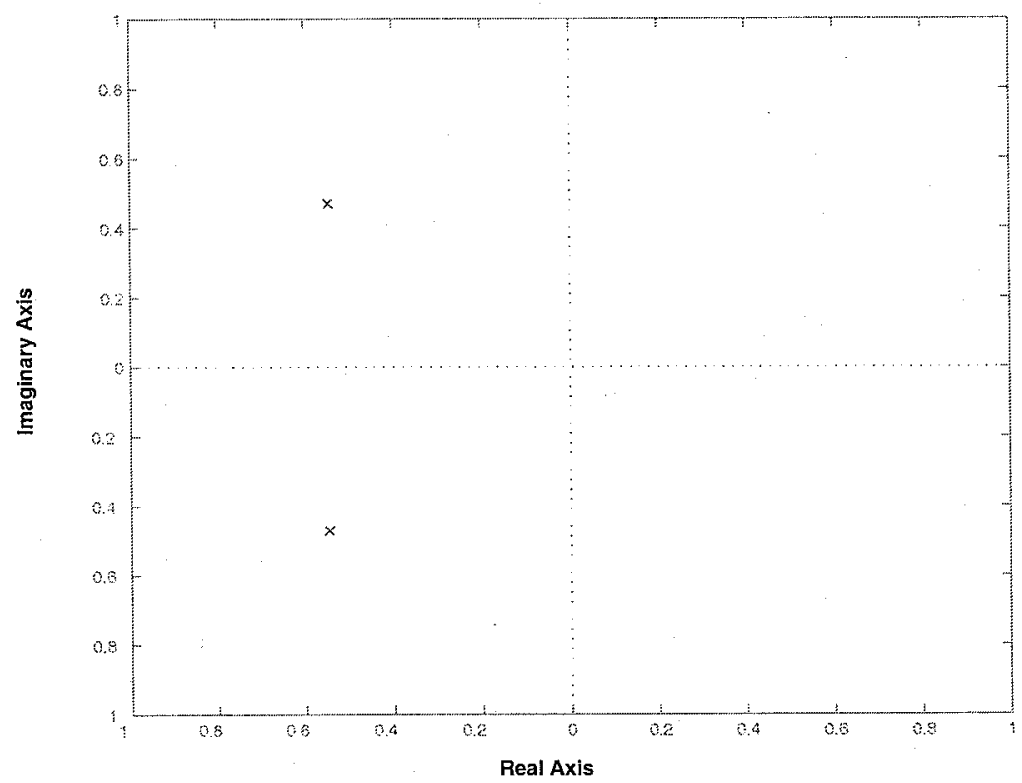

Figure 9-3: $G_{\theta}$ Pole-Zero Plot

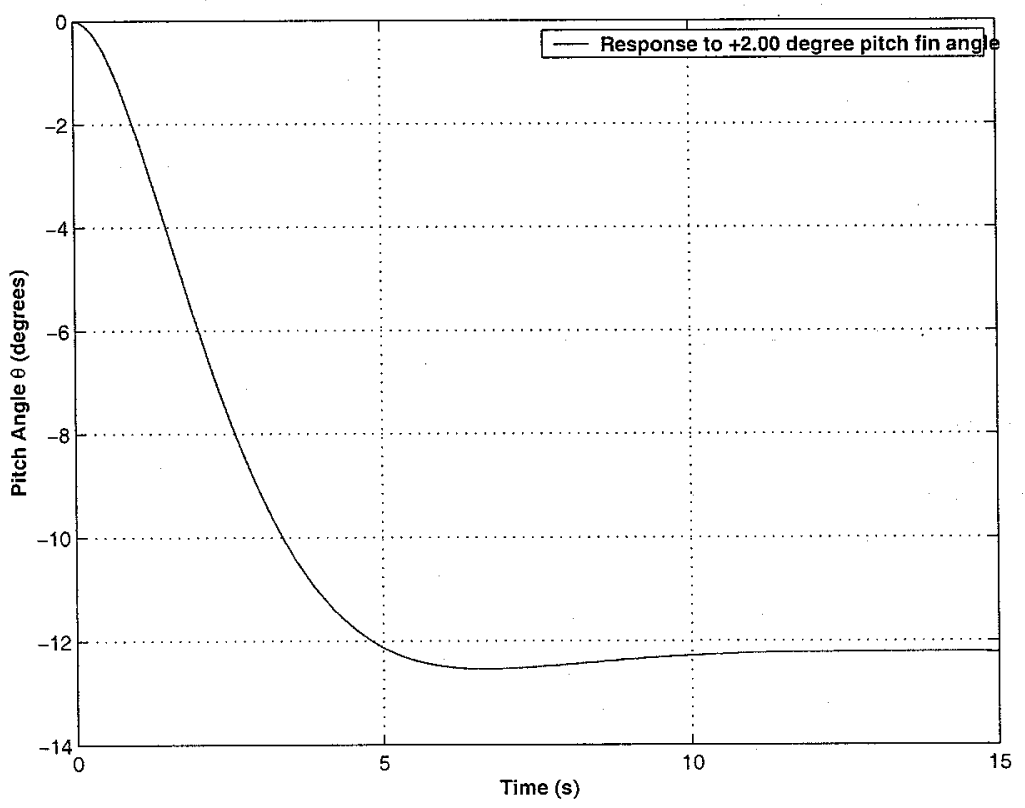

Figure 9-4: $G_{\theta}$ Open-Loop Step Response 


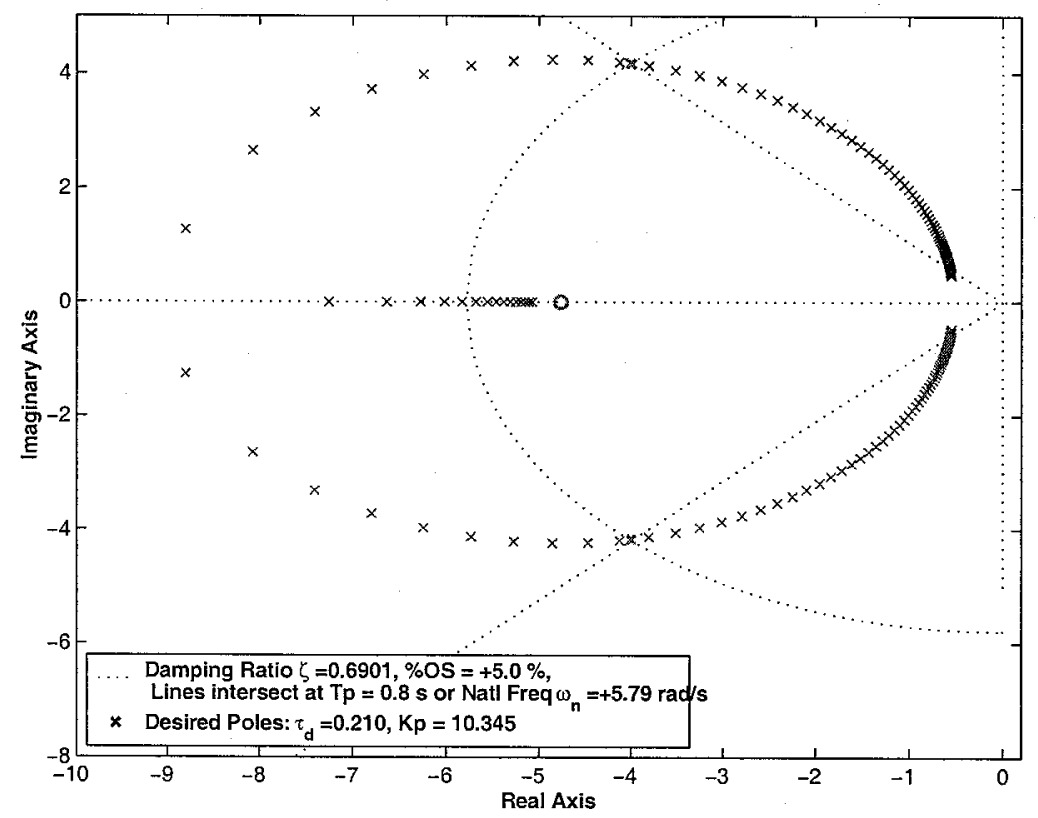

Figure 9-5: $G_{\theta}$ Root-Locus Plot

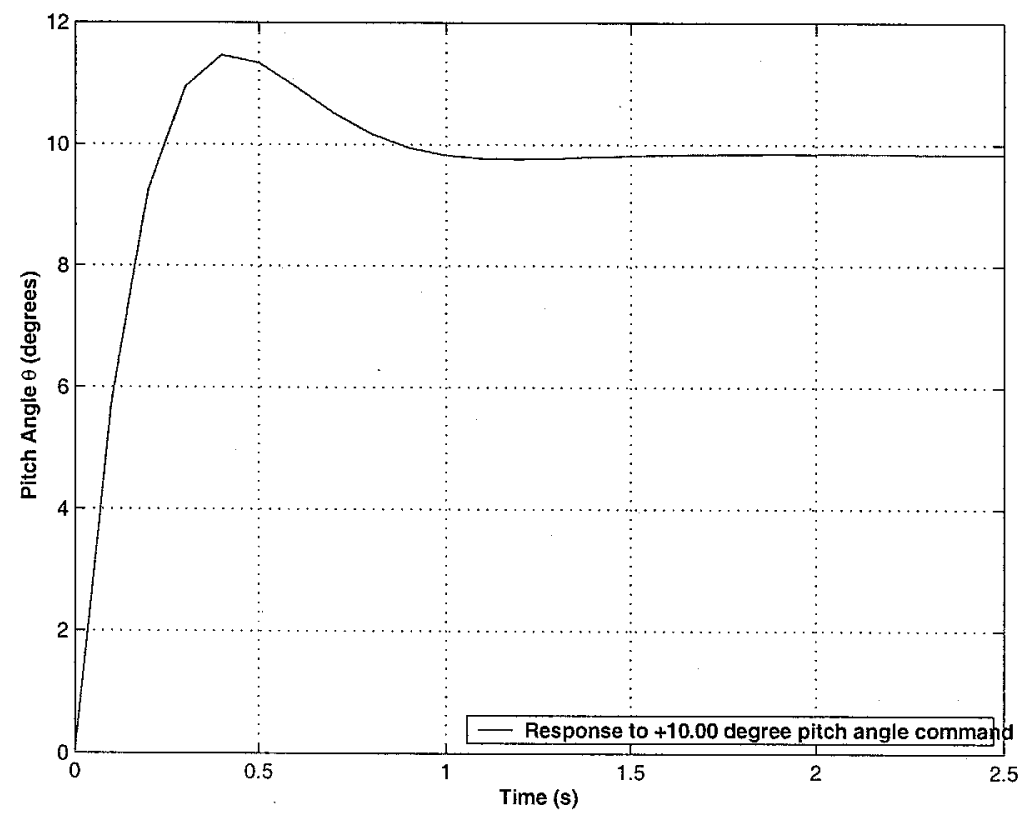

Figure 9-6: $G_{\theta}$ Closed-Loop Step Response 


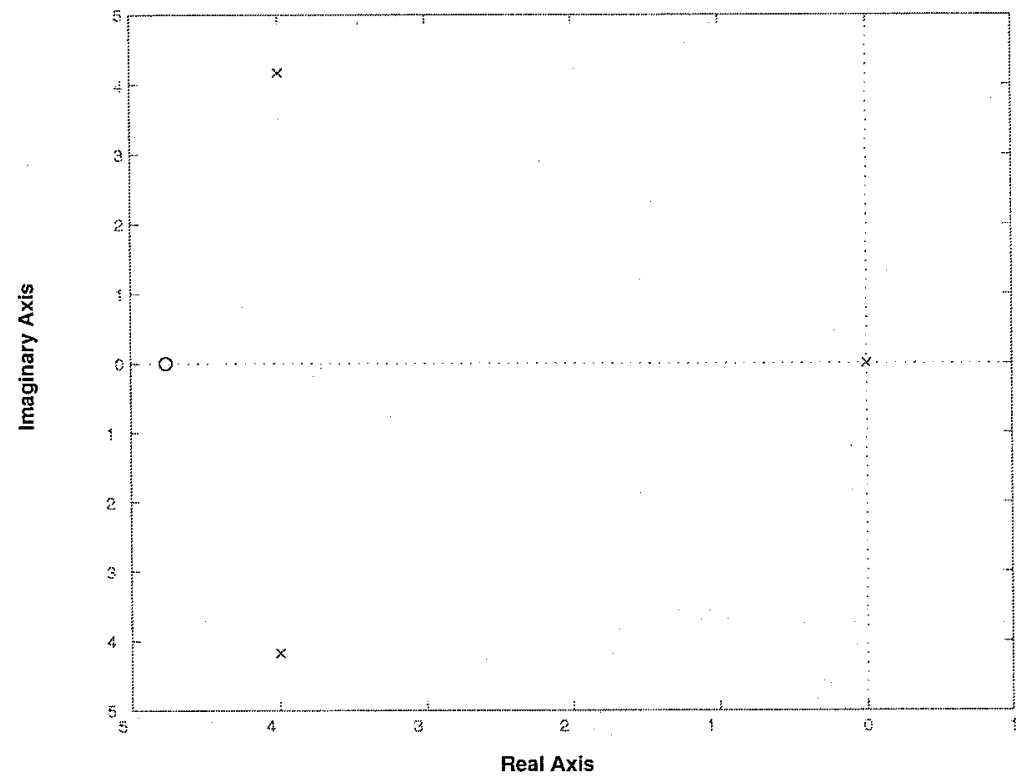

Figure 9-7: $G_{z} * H_{\theta}$ Pole-Zero Plot

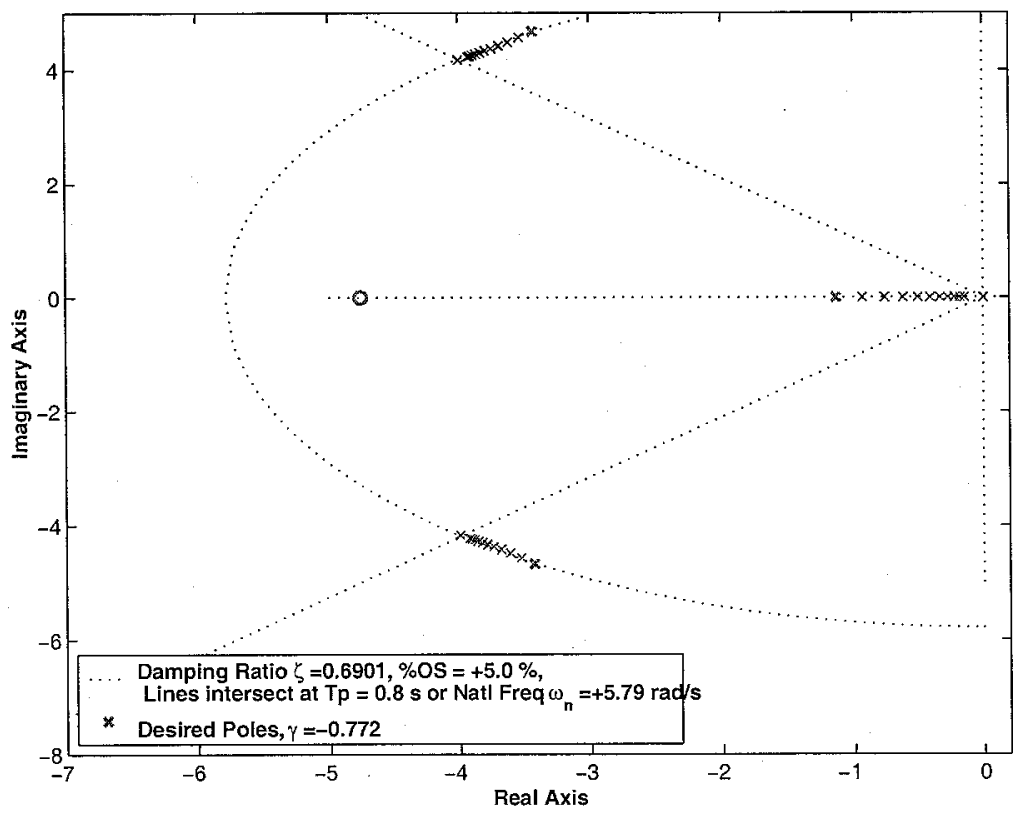

Figure 9-8: $G_{z} * H_{\theta}$ Root-Locus Plot 
a simulation. In Figure 9-11, the model is run without disturbances or actuator saturation. Notice that the controller commands nonsensical vehicle fin angles in excess of 180 degrees. In reality, the vehicle fins stall at an angle of attack less than 15 degrees.

In Figure 9-14, the model incorporates fin angle saturation, random pitch sensor noise, and the effects of a 1 knot vertical current. Notice that the vehicle response is slower, and that the fins exhibit a considerable amount of "flutter".

\subsection{Controller Implementation}

In this chapter we have shown the development of vehicle depth control system based on a linearized model of the vehicle dynamics. Although in simulation the controller appears to achieve the desired response, it remains to be seen if it would work as well on the actual vehicle.

The next step in testing the controller would be to replace the linearized depth-plane plant in Figure 9-10 with the non-linear, six degree of freedom vehicle model developed in the earlier chapters. This would allow us to gauge how well the controller handles vehicle behavior outside the linearized regime of small angles and small accelerations.

The final step would be to test the depth-plane controller on the actual vehicle at sea. 


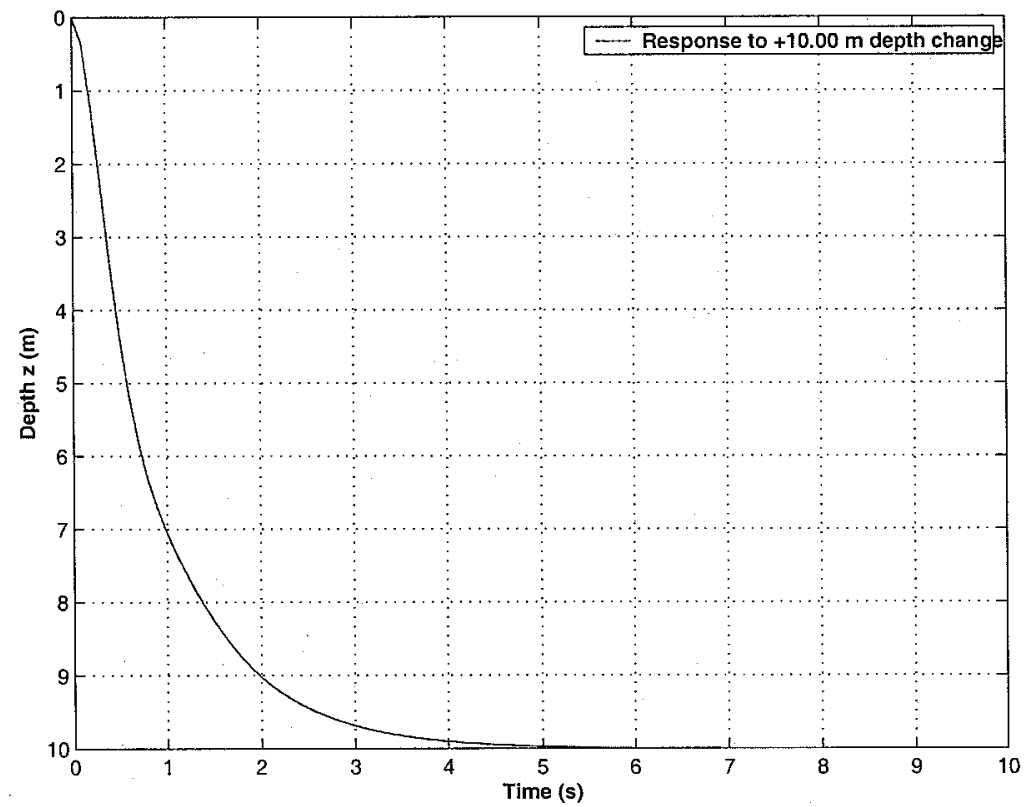

Figure 9-9: $G_{z} * H_{\theta}$ Closed-Loop Step Response

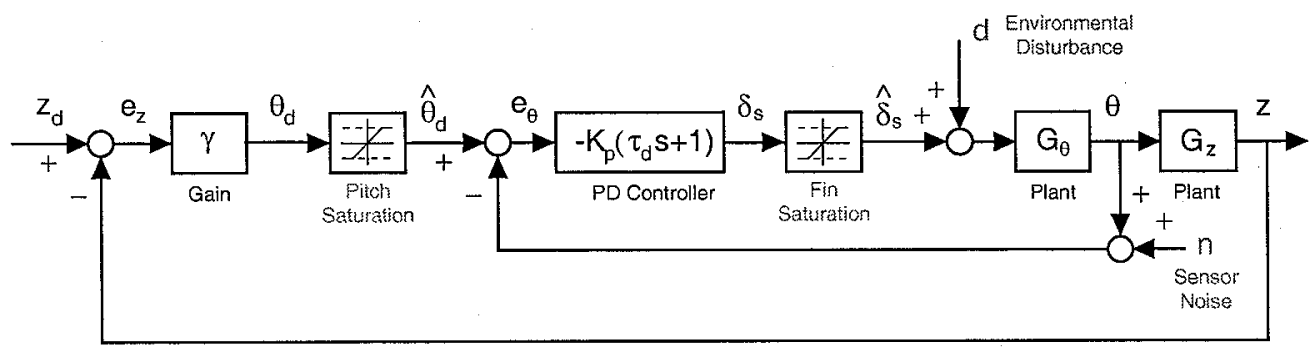

Figure 9-10: Depth-Plane Control System Block Diagram with Actuator Limits, Environmental Disturbance, and Sensor Noise 

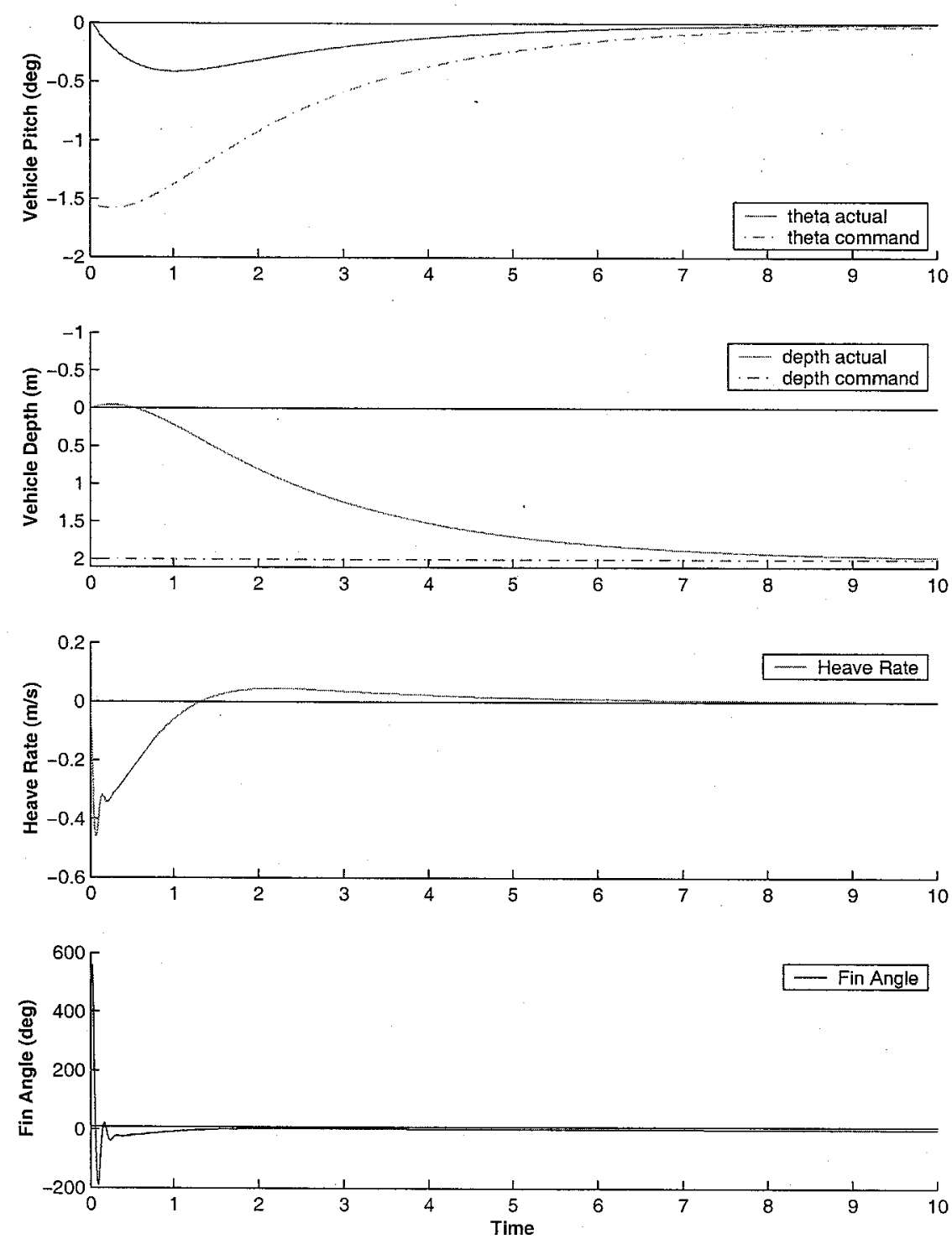

Figure 9-11: Vehicle Simulation, based on basic block diagram 

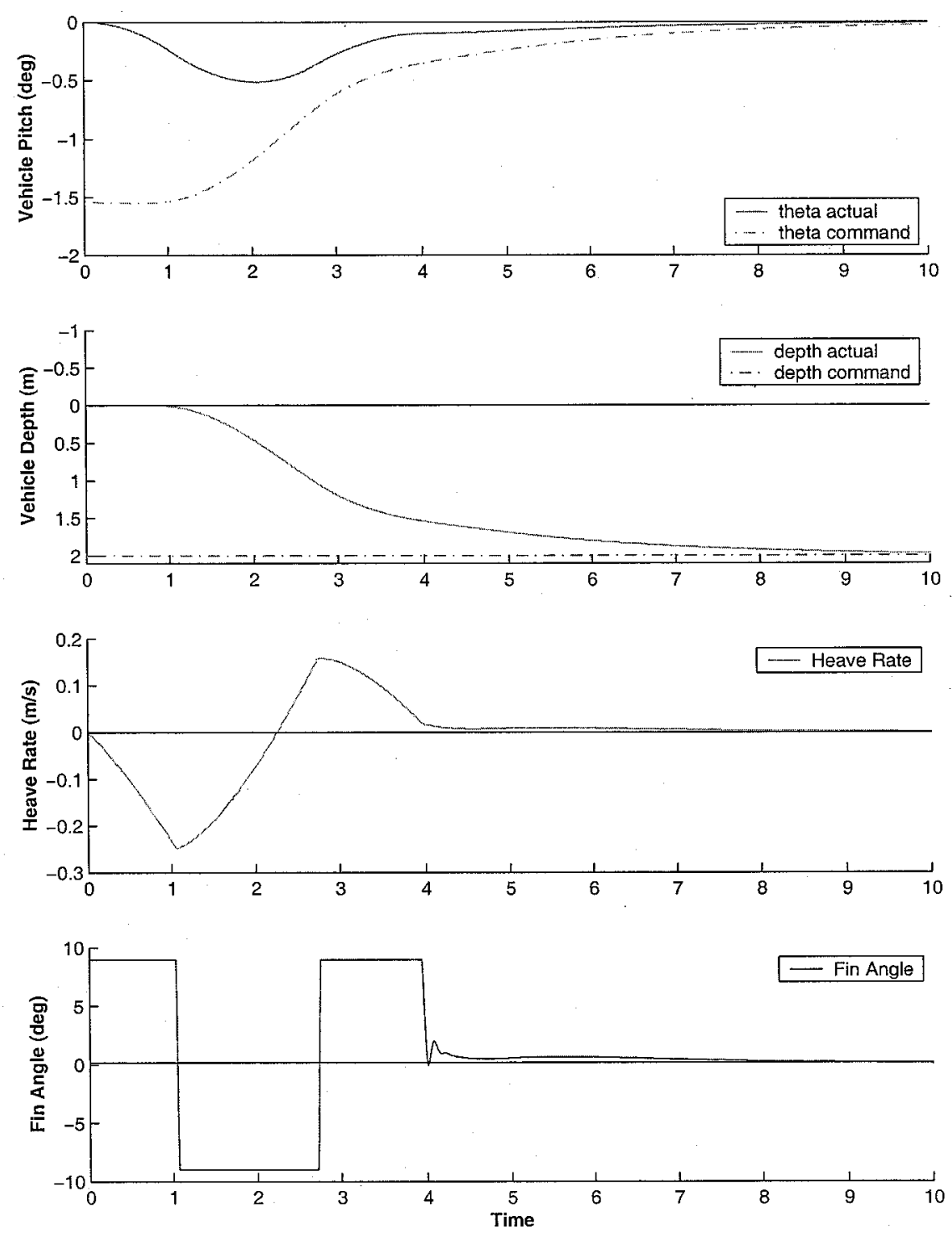

Figure 9-12: Vehicle Simulation, with fin angle saturation 

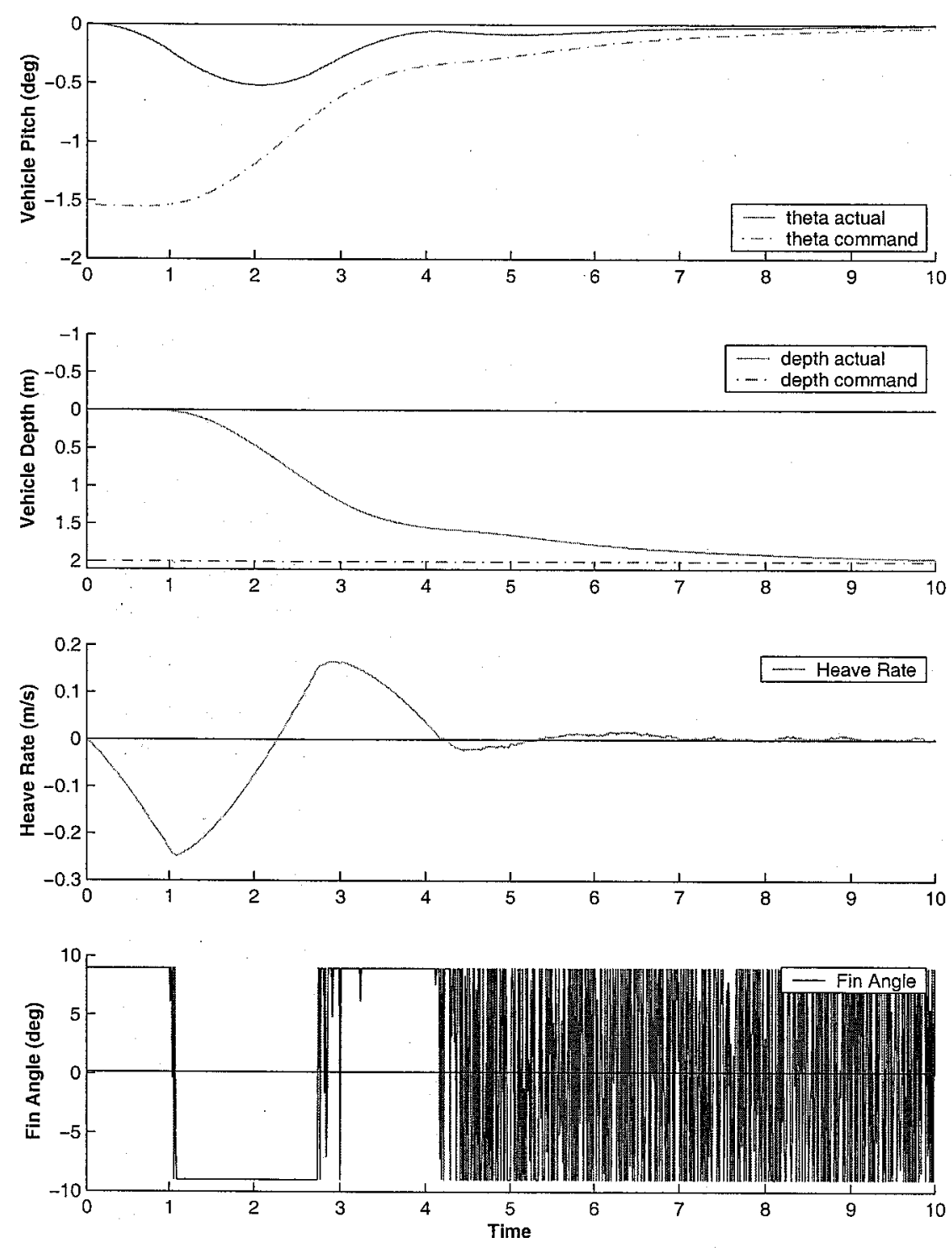

Figure 9-13: Vehicle Simulation, with fin angle saturation and random pitch sensor noise 

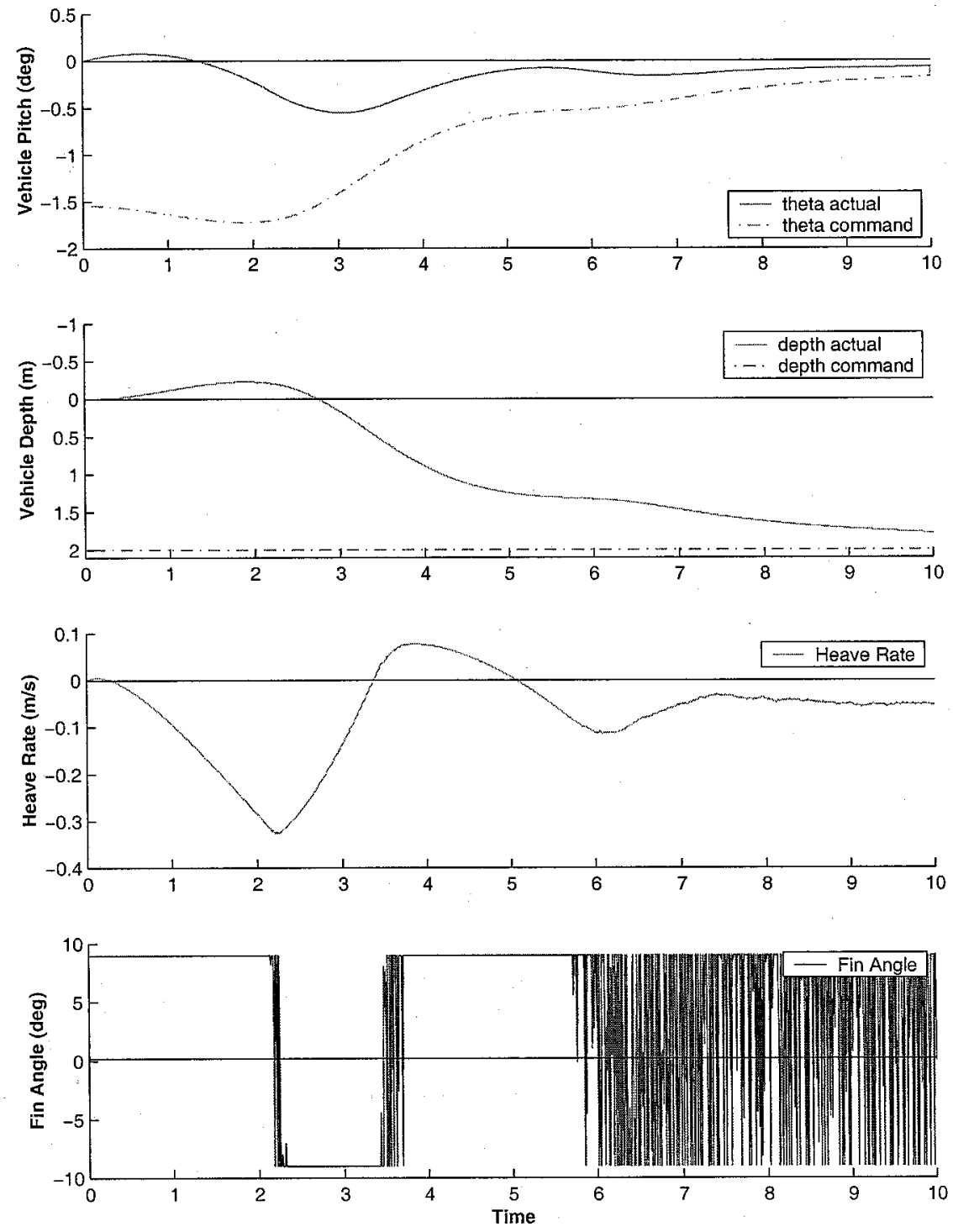

Figure 9-14: Vehicle Simulation, incorporating fin angle saturation, random pitch sensor noise, and 1 knot vertical current 


\section{Chapter 10}

\section{Conclusion}

In the preceding chapters, we have demonstrated the development of a mathematical model for the dynamics of an autonomous underwater vehicle. We have examined methods for validating the performance of this model. Finally, we have seen how such a model can be applied to the development of a vehicle control system.

In the following sections, we outline a series of recommendations for expanding upon this work.

\subsection{Expanded Tow Tank Measurements}

In the previous chapter, the author outlined the limitations of vehicle coefficients based solely upon semi-empirical formulae. The most significant source of error is in the way coefficients are used to model the vehicle moving at some angle of attack, as shown in Figure 10-1. The fluid effects are

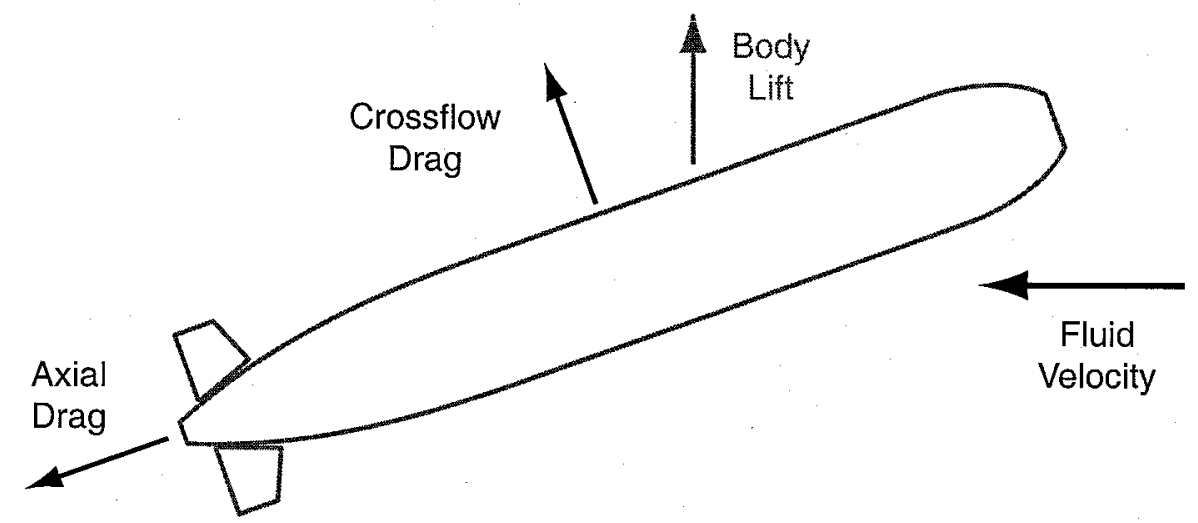

Figure 10-1: Forces on the vehicle at an angle of attack

broken up into vehicle body lift, vehicle crossflow drag, and vehicle axial drag. Of these, the author had the most difficulty estimating body lift.

The semi-empirical methods of calculating the body lift coefficient used by Hoerner [16], Bottaccini [7], and Nahon [23], explored in the development of this thesis, were found to differ by an order of magnitude. It will therefore be important to experimentally measure forces and moments on the vehicle moving at an angle of attack, in order to verify the empirical estimates. 


\subsection{Future Experiments at Sea}

The following are recommendations for future experiments at sea, derived from the author's experience and consultation with experts in the field.

\subsubsection{Improved Vehicle Instrumentation}

The vehicle dynamics data collected in the experiments described in Chapter 7 were limited in the number of vehicle states recorded, and the accuracy of those measurements. This made it extremely difficult to judge the validity of the model comparison.

For future experiments, the author has augmented the standard REMUS sensors described in Section 7.3 with an inertial measurement unit: the Crossbow DMU-AHRS (Dynamic Measurement Unit-Attitude and Heading Reference System). This instrument outputs magnetic orientation, accelerations and angular rates on three axes. From this instrument, we will be able to accurately measure or derive the following vehicle states.

$$
\boldsymbol{x}=\left[\begin{array}{llllllllllll}
\dot{u} & \dot{v} & \dot{w} & u & v & w & p & q & r & \phi & \theta & \psi
\end{array}\right]^{T}
$$

This will significantly improve our ability to measure the vehicle initial conditions in particular, and the vehicle motion in general.

\subsubsection{Measurement of Vehicle Parameters}

With the exception of the longitudinal center of gravity $x_{c g}$, which was measured before each experiment using a balance, the author's estimates for the vehicle mass distribution were calculated using the vehicle weight list. Similarly, the estimates for the vehicle center of buoyancy were calculated using the Myring hull shape.

To reduce uncertainty in future experiments, it will be necessary to measure these values experimentally, preferably before each experiment.

\subsubsection{Isolation of Vehicle Motion}

One limitation of the experimental step response data used in the model validation was imperfect knowledge of the vehicle initial conditions. At the time of the experiments, the author had no way of measuring the vehicle accelerations and angular rates prior to the changes in fin angles.

These vehicle motions resulted from two sources: vehicle control inputs and external disturbances. In future experiments, every effort should be made to minimize these motions.

To minimize vehicle control motions, it is important to understand the vehicle steady-state conditions. AUVs like REMUS can be unstable when operating without control. Using the inertial measurement unit, it will be possible to identify the propeller RPM and fixed fin angles which result in straight and level vehicle flight. These settings should then be used at the start of every experimental run.

To minimize environmental disturbances, the experiments could be run in an area known to be free of currents, and at sufficient depth to avoid free surface interactions. Two locations meeting these criteria would be deep lakes and flooded sinkholes.

\subsection{Controller-Based Model Comparison}

The vehicle model is particularly useful as a tool in developing vehicle control systems. To that end, rather than comparing the model output to vehicle data collected during open-loop maneuvers, it would be more useful to compare the model to the vehicle behavior during closed-loop control. This 
would mean incorporating the vehicle controller as the interface to the model code. Rather than actuator states such as the vehicle fin angles $\delta_{s}$ and deltar, the model inputs would instead be the commanded states, such as desired depth $z_{d}$ or desired heading $\psi_{d}$.

\subsection{Vehicle Sensor Model}

Developing a vehicle sensor model would enable us to improve the vehicle performance without necessarily improving the vehicle sensors. As part of the vehicle tow tank experiments and experiments at sea, precision vehicle inertial measurements could be used to calibrate the other vehicle sensors and estimate their dynamic response.

\subsection{Improved Coefficient-Based Model}

Mission planning for the shallow water operation of AUVs depends on an accurate knowledge of the performance limits of these vehicles in terms of water depth and sea state. A map of this twodimensional space for shallow water is illustrated in Figure 10-2. At present, these limits are not known.

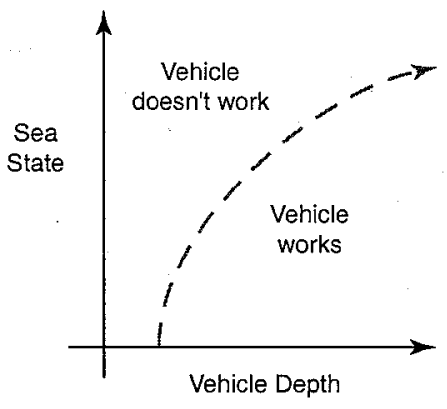

Figure 10-2: Vehicle performance limits as a function of depth and sea state

A vehicle model based on vehicle state- and environment-dependent transfer functions could prove an effective method for simulating the dynamics of underwater vehicles near the surf zone. The transfer functions for such a vehicle model would themselves be functions of the operating state of the vehicle, the water depth and the sea state.

These transfer functions could be derived from a combination of existing data sets for AUVs in shallow water and waves $[4,27,33]$ and numerical vehicle model codes $[26,13,34,21]$.

Given such a model and a method for simulating the disturbances caused by a random wave field, one could predict the probabilistic deviation of an underwater vehicle from a given desired trajectory. Control system designers and mission planners could use this stochastic analysis to determine the operating limits of their vehicles. 


\section{Appendix A}

\section{Tables of Parameters}

Table A.1: STD REMUS Hull Parameters

\begin{tabular}{cccl}
\hline \hline Parameter & Value & Units & Description \\
\hline$\rho$ & $+1.03 \mathrm{e}+003$ & $\mathrm{~kg} / \mathrm{m}^{3}$ & Seawater Density \\
$A_{f}$ & $+2.85 \mathrm{e}-002$ & $\mathrm{~m}^{2}$ & Hull Frontal Area \\
$A_{p}$ & $+2.26 \mathrm{e}-001$ & $\mathrm{~m}^{2}$ & Hull Projected Area (xz plane) \\
$S_{w}$ & $+7.09 \mathrm{e}-001$ & $\mathrm{~m}^{2}$ & Hull Wetted Surface Area \\
$\nabla$ & $+3.15 \mathrm{e}-002$ & $\mathrm{~m}^{3}$ & Estimated Hull Volume \\
$W$ & $+2.99 \mathrm{e}+002$ & $\mathrm{~N}$ & Measured Vehicle Weight \\
$B$ & $+3.08 \mathrm{e}+002$ & $\mathrm{~N}$ & Measured Vehicle Buoyancy \\
$B_{\text {est }}$ & $+3.17 \mathrm{e}+002$ & $\mathrm{~N}$ & Estimated Hull Buoyancy \\
$x_{\mathrm{cb}(\mathrm{est})}$ & $+5.54 \mathrm{e}-003$ & $\mathrm{~m}$ & Est. Long. Center of Buoyancy \\
$c_{\mathrm{d}}$ & $+3.00 \mathrm{e}-001$ & $\mathrm{n} / \mathrm{a}$ & REMUS Axial Drag Coeff. \\
$c_{\mathrm{dc}}$ & $+1.10 \mathrm{e}+000$ & $\mathrm{n} / \mathrm{a}$ & Cylinder Crossflow Drag Coeff. \\
$c_{\mathrm{yd} \beta}$ & $+1.20 \mathrm{e}+000$ & $\mathrm{n} / \mathrm{a}$ & Hoerner Body Lift Coeff. \\
$x_{\mathrm{cp}}$ & $-3.21 \mathrm{e}-001$ & $\mathrm{n} / \mathrm{a}$ & Center of Pressure \\
$\alpha$ & $+3.59 \mathrm{e}-002$ & $\mathrm{n} / \mathrm{a}$ & Ellipsoid Added Mass Coeff. \\
\hline \hline
\end{tabular}

Table A.2: Hull Coordinates for Limits of Integration

\begin{tabular}{cccl}
\hline \hline Parameter & Value & Units & Description \\
\hline$x_{\mathrm{t}}$ & $-7.21 \mathrm{e}-001$ & $\mathrm{~m}$ & Aft End of Tail Section \\
$x_{\mathrm{t} 2}$ & $-2.18 \mathrm{e}-001$ & $\mathrm{~m}$ & Forward End of Tail Section \\
$x_{\mathrm{f}}$ & $-6.85 \mathrm{e}-001$ & $\mathrm{~m}$ & Aft End of Fin Section \\
$x_{\mathrm{f} 2}$ & $-6.11 \mathrm{e}-001$ & $\mathrm{~m}$ & Forward End of Fin Section \\
$x_{\mathrm{b}}$ & $+4.37 \mathrm{e}-001$ & $\mathrm{~m}$ & Aft End of Bow Section \\
$x_{\mathrm{b} 2}$ & $+6.10 \mathrm{e}-001$ & $\mathrm{~m}$ & Forward End of Bow Section \\
\hline \hline
\end{tabular}


Table A.3: Center of Buoyancy wrt Origin at Vehicle Nose

\begin{tabular}{ccc}
\hline \hline Parameter & Value & Units \\
\hline$x_{\mathrm{cb}}$ & $-6.11 \mathrm{e}-001$ & $\mathrm{~m}$ \\
$y_{\mathrm{cb}}$ & $+0.00 \mathrm{e}+000$ & $\mathrm{~m}$ \\
$z_{\mathrm{cb}}$ & $+0.00 \mathrm{e}+000$ & $\mathrm{~m}$ \\
\hline \hline
\end{tabular}

Table A.4: Center of Gravity wrt Origin at CB

\begin{tabular}{ccc}
\hline Parameter & Value & Units \\
\hline$x_{\mathrm{cg}}$ & $+0.00 \mathrm{e}+000$ & $\mathrm{~m}$ \\
$y_{\mathrm{cg}}$ & $+0.00 \mathrm{e}+000$ & $\mathrm{~m}$ \\
$z_{\mathrm{cg}}$ & $+1.96 \mathrm{e}-002$ & $\mathrm{~m}$ \\
\hline \hline
\end{tabular}

Table A.5: REMUS Fin Parameters

\begin{tabular}{cccl}
\hline \hline Parameter & Value & Units & Description \\
\hline$S_{\text {fin }}$ & $+6.65 e-003$ & $\mathrm{~m}^{2}$ & Planform Area \\
$b_{\text {fin }}$ & $+8.57 e-002$ & $\mathrm{~m}$ & Span \\
$x_{\text {finpost }}$ & $-6.38 \mathrm{e}-001$ & $\mathrm{~m}$ & Moment Arm wrt Vehicle Origin at CB \\
$\delta_{\max }$ & $+1.36 \mathrm{e}+001$ & $\mathrm{deg}$ & Maximum Fin Angle \\
$a_{\mathrm{fin}}$ & $+5.14 \mathrm{e}+000$ & $\mathrm{~m}$ & Max Fin Height Above Centerline \\
$c_{\text {mean }}$ & $+7.47 \mathrm{e}-002$ & $\mathrm{~m}$ & Mean Chord Length \\
$t$ & $+6.54 \mathrm{e}-001$ & $\mathrm{n} / \mathrm{a}$ & Fin Taper Ratio (Whicker-Felner) \\
$c_{\mathrm{df}}$ & $+5.58 \mathrm{e}-001$ & $\mathrm{n} / \mathrm{a}$ & Fin Crossflow Drag Coefficient \\
$\mathrm{AR}$ & $+2.21 \mathrm{e}+000$ & $\mathrm{n} / \mathrm{a}$ & Effective Aspect Ratio \\
$\bar{a}$ & $+9.00 \mathrm{e}-001$ & $\mathrm{n} / \mathrm{a}$ & Lift Slope Parameter \\
$c_{L \alpha}$ & $+3.12 \mathrm{e}+000$ & $\mathrm{n} / \mathrm{a}$ & Fin Lift Slope \\
\hline \hline
\end{tabular}




\section{Appendix B}

\section{Tables of Combined Non-Linear Coefficients}

Note that all coefficients are calculated for the STD REMUS hull profile. Unlike those given in Tables 4.2 and 4.3, these values include the correction factors described in Section 8.1.2.

Table B.1: Non-Linear Force Coefficients

\begin{tabular}{cccl}
\hline \hline Parameter & Value & Units & Description \\
\hline$X_{u u}$ & $-1.62 \mathrm{e}+000$ & $\mathrm{~kg} / \mathrm{m}$ & Cross-flow Drag \\
$X_{\dot{u}}$ & $-9.30 \mathrm{e}-001$ & $\mathrm{~kg}$ & Added Mass \\
$X_{w q}$ & $-3.55 \mathrm{e}+001$ & $\mathrm{~kg} / \mathrm{rad}$ & Added Mass Cross-term \\
$X_{q q}$ & $-1.93 \mathrm{e}+000$ & $\mathrm{~kg} \cdot \mathrm{m} / \mathrm{rad}$ & Added Mass Cross-term \\
$X_{v r}$ & $+3.55 \mathrm{e}+001$ & $\mathrm{~kg} / \mathrm{rad}$ & Added Mass Cross-term \\
$X_{r r}$ & $-1.93 \mathrm{e}+000$ & $\mathrm{~kg} \cdot \mathrm{m} / \mathrm{rad}$ & Added Mass Cross-term \\
$X_{p r o p}$ & $+3.86 \mathrm{e}+000$ & $\mathrm{~N}$ & Propeller Thrust \\
$Y_{v v}$ & $-1.31 \mathrm{e}+003$ & $\mathrm{~kg} / \mathrm{m}$ & Cross-flow Drag \\
$Y_{r r}$ & $+6.32 \mathrm{e}-001$ & $\mathrm{~kg} \cdot \mathrm{m} / \mathrm{rad}$ & Cross-flow Drag \\
$Y_{u v}$ & $-2.86 \mathrm{e}+001$ & $\mathrm{~kg} / \mathrm{m}$ & Body Lift Force and Fin Lift \\
$Y_{\dot{v}}$ & $-3.55 \mathrm{e}+001$ & $\mathrm{~kg}$ & Added Mass \\
$Y_{\dot{r}}$ & $+1.93 \mathrm{e}+000$ & $\mathrm{~kg} \cdot \mathrm{m} / \mathrm{rad}$ & Added Mass \\
$Y_{u r}$ & $+5.22 \mathrm{e}+000$ & $\mathrm{~kg} / \mathrm{rad}$ & Added Mass Cross Term and Fin Lift \\
$Y_{w p}$ & $+3.55 \mathrm{e}+001$ & $\mathrm{~kg} / \mathrm{rad}$ & Added Mass Cross-term \\
$Y_{p q}$ & $+1.93 \mathrm{e}+000$ & $\mathrm{~kg} \cdot \mathrm{m} / \mathrm{rad}$ & Added Mass Cross-term \\
$Y_{u u d r}$ & $+9.64 \mathrm{e}+000$ & $\mathrm{~kg} /(\mathrm{m} \cdot \mathrm{rad})$ & Fin Lift Force \\
$Z_{w w}$ & $-1.31 \mathrm{e}+002$ & $\mathrm{~kg} / \mathrm{m}$ & Cross-flow Drag \\
$Z_{q q}$ & $-6.32 \mathrm{e}-001$ & $\mathrm{~kg} \cdot \mathrm{m} / \mathrm{rad}$ & Cross-flow Drag \\
$Z_{u w}$ & $-2.86 \mathrm{e}+001$ & $\mathrm{~kg} / \mathrm{m}$ & Body Lift Force and Fin Lift \\
$Z_{w}$ & $-3.55 \mathrm{e}+001$ & $\mathrm{~kg}$ & Added Mass \\
$Z_{\dot{q}}$ & $-1.93 \mathrm{e}+000$ & $\mathrm{~kg} \cdot \mathrm{m} / \mathrm{rad}$ & Added Mass \\
$Z_{u q}$ & $-5.22 \mathrm{e}+000$ & $\mathrm{~kg} / \mathrm{rad}$ & Added Mass Cross-term and Fin Lift \\
$Z_{v p}$ & $-3.55 \mathrm{e}+001$ & $\mathrm{~kg} / \mathrm{rad}$ & Added Mass Cross-term \\
$Z_{r p}$ & $+1.93 \mathrm{e}+000$ & $\mathrm{~kg} / \mathrm{rad}$ & Added Mass Cross-term \\
$Z_{u u d s}$ & $-9.64 \mathrm{e}+000$ & $\mathrm{~kg} /(\mathrm{m} \cdot \mathrm{rad})$ & Fin Lift Force \\
\hline \hline
\end{tabular}


Table B.2: Non-Linear Moment Coefficients

\begin{tabular}{cccl}
\hline \hline Parameter & Value & Units & Description \\
\hline$K_{p p}$ & $-1.30 \mathrm{e}-001$ & $\mathrm{~kg} \cdot \mathrm{m}^{2} / \mathrm{rad}^{2}$ & Rolling Resistance \\
$K_{\dot{p}}$ & $-7.04 \mathrm{e}-002$ & $\mathrm{~kg} \cdot \mathrm{m}^{2} / \mathrm{rad}$ & Added Mass \\
$K_{p r o p}$ & $-5.43 \mathrm{e}-001$ & $\mathrm{~N} \cdot \mathrm{m}$ & Propeller Torque \\
$M_{w w}$ & $+3.18 \mathrm{e}+000$ & $\mathrm{~kg}$ & Cross-flow Drag \\
$M_{q q}$ & $-1.88 \mathrm{e}+002$ & $\mathrm{~kg} \cdot \mathrm{m}^{2} / \mathrm{rad}^{2}$ & Cross-flow Drag \\
$M_{u w}$ & $+2.40 \mathrm{e}+001$ & $\mathrm{~kg}$ & Body and Fin Lift and Munk Moment \\
$M_{w}$ & $-1.93 \mathrm{e}+000$ & $\mathrm{~kg} \cdot \mathrm{m}$ & Added Mass \\
$M_{\dot{q}}$ & $-4.88 \mathrm{e}+000$ & $\mathrm{~kg} \cdot \mathrm{m}^{2} / \mathrm{rad}$ & Added Mass \\
$M_{u q}$ & $-2.00 \mathrm{e}+000$ & $\mathrm{~kg} \cdot \mathrm{m} / \mathrm{rad}$ & Added Mass Cross Term and Fin Lift \\
$M_{v p}$ & $-1.93 \mathrm{e}+000$ & $\mathrm{~kg} \cdot \mathrm{m} / \mathrm{rad}$ & Added Mass Cross Term \\
$M_{r p}$ & $+4.86 \mathrm{e}+000$ & $\mathrm{~kg} \cdot \mathrm{m}^{2} / \mathrm{rad}$ & Added Mass Cross-term \\
$M_{u u d s}$ & $-6.15 \mathrm{e}+000$ & $\mathrm{~kg} / \mathrm{rad}$ & Fin Lift Moment \\
$N_{v v}$ & $-3.18 \mathrm{e}+000$ & $\mathrm{~kg}$ & Cross-flow Drag \\
$N_{r r}$ & $-9.40 \mathrm{e}+001$ & $\mathrm{~kg} \cdot \mathrm{m}^{2} / \mathrm{rad}$ & Cross-flow Drag \\
$N_{u v}$ & $-2.40 \mathrm{e}+001$ & $\mathrm{~kg}$ & Body and Fin Lift and Munk Moment \\
$N_{\dot{v}}$ & $+1.93 \mathrm{e}+000$ & $\mathrm{~kg} \cdot \mathrm{m}$ & Added Mass \\
$N_{\dot{r}}$ & $-4.88 \mathrm{e}+000$ & $\mathrm{~kg} \cdot \mathrm{m}^{2} / \mathrm{rad}$ & Added Mass \\
$N_{u r}$ & $-2.00 \mathrm{e}+000$ & $\mathrm{~kg} \cdot \mathrm{m} / \mathrm{rad}$ & Added Mass Cross Term and Fin Lift \\
$N_{w p}$ & $-1.93 \mathrm{e}+000$ & $\mathrm{~kg} \cdot \mathrm{m} / \mathrm{rad}$ & Added Mass Cross Term \\
$N_{p q}$ & $-4.86 \mathrm{e}+000$ & $\mathrm{~kg} \cdot \mathrm{m}^{2} / \mathrm{rad}$ & Added Mass Cross-term \\
$N_{u u d r}$ & $-6.15 \mathrm{e}+000$ & $\mathrm{~kg} / \mathrm{rad}$ & Fin Lift Moment \\
\hline \hline
\end{tabular}




\section{Appendix C}

\section{Tables of Non-Linear Coefficients by Type}

Note that all coefficients are calculated for the STD REMUS hull profile. Unlike those given in Tables 4.2 and 4.3, these values include the correction factors described in Section 8.1.2.

\begin{tabular}{ccc}
\multicolumn{3}{c}{ Table C.1: Axial Drag Coefficient } \\
\hline \hline Parameter & Value & Units \\
\hline$X_{u u}$ & $-1.62 \mathrm{e}+000$ & $\mathrm{~kg} / \mathrm{m}$ \\
\hline \hline
\end{tabular}

Table C.2: Crossflow Drag Coefficients

\begin{tabular}{ccc}
\hline \hline Parameter & Value & Units \\
\hline$Y_{v v}$ & $-1.31 \mathrm{e}+003$ & $\mathrm{~kg} / \mathrm{m}$ \\
$Y_{r r d}$ & $+6.32 \mathrm{e}-001$ & $\mathrm{~kg} \cdot \mathrm{m} / \mathrm{rad}^{2}$ \\
$Z_{w w}$ & $-1.31 \mathrm{e}+002$ & $\mathrm{~kg} / \mathrm{m}$ \\
$Z_{q q d}$ & $-6.32 \mathrm{e}-001$ & $\mathrm{~kg} \cdot \mathrm{m} / \mathrm{rad}^{2}$ \\
$M_{w w d}$ & $+3.18 \mathrm{e}+000$ & $\mathrm{~kg}$ \\
$M_{q q}$ & $-1.88 \mathrm{e}+002$ & $\mathrm{~kg} \cdot \mathrm{m}^{2} / \mathrm{rad}^{2}$ \\
$N_{v v d}$ & $-3.18 \mathrm{e}+000$ & $\mathrm{~kg}$ \\
$N_{r r}$ & $-9.40 \mathrm{e}+001$ & $\mathrm{~kg} \cdot \mathrm{m}^{2} / \mathrm{rad}^{2}$ \\
\hline \hline
\end{tabular}

Table C.3: Rolling Resistance Coefficient

\begin{tabular}{ccc}
\hline \hline Parameter & Value & Units \\
\hline$K_{p p}$ & $-1.30 \mathrm{e}-001$ & $\mathrm{~kg} \cdot \mathrm{m}^{2} / \mathrm{rad}^{2}$ \\
\hline \hline
\end{tabular}


Table C.4: Body Lift and Moment Coefficients

\begin{tabular}{ccc}
\hline \hline Parameter & Value & Units \\
\hline$Y_{u v}$ & $-2.86 \mathrm{e}+001$ & $\mathrm{~kg} / \mathrm{m}$ \\
$Z_{u w}$ & $-2.86 \mathrm{e}+001$ & $\mathrm{~kg} / \mathrm{m}$ \\
$M_{u w b}$ & $-4.47 \mathrm{e}+000$ & $\mathrm{~kg}$ \\
$N_{u v b}$ & $+4.47 \mathrm{e}+000$ & $\mathrm{~kg}$ \\
\hline \hline
\end{tabular}

Table C.5: Added Mass Coefficients

\begin{tabular}{ccc}
\hline \hline Parameter & Value & $\mathrm{Units}$ \\
\hline$X_{\dot{u}}$ & $-9.30 \mathrm{e}-001$ & $\mathrm{~kg}$ \\
$X_{\dot{v}}$ & $+0.00 \mathrm{e}+000$ & $\mathrm{~kg}$ \\
$X_{\dot{w}}$ & $+0.00 \mathrm{e}+000$ & $\mathrm{~kg}$ \\
$X_{\dot{p}}$ & $+0.00 \mathrm{e}+000$ & $\mathrm{~kg} \cdot \mathrm{m} / \mathrm{rad}$ \\
$X_{\dot{q}}$ & $+0.00 \mathrm{e}+000$ & $\mathrm{~kg} \cdot \mathrm{m} / \mathrm{rad}$ \\
$X_{\dot{r}}$ & $+0.00 \mathrm{e}+000$ & $\mathrm{~kg} \cdot \mathrm{m} / \mathrm{rad}$ \\
$Y_{\dot{u}}$ & $+0.00 \mathrm{e}+000$ & $\mathrm{~kg}$ \\
$Y_{\dot{v}}$ & $-3.55 \mathrm{e}+001$ & $\mathrm{~kg}$ \\
$Y_{\dot{w}}$ & $+0.00 \mathrm{e}+000$ & $\mathrm{~kg}$ \\
$Y_{\dot{p}}$ & $+0.00 \mathrm{e}+000$ & $\mathrm{~kg} \cdot \mathrm{m} / \mathrm{rad}$ \\
$Y_{\dot{q}}$ & $+0.00 \mathrm{e}+000$ & $\mathrm{~kg} \cdot \mathrm{m} / \mathrm{rad}$ \\
$Y_{\dot{r}}$ & $+1.93 \mathrm{e}+000$ & $\mathrm{~kg} \cdot \mathrm{m} / \mathrm{rad}$ \\
$Z_{\dot{u}}$ & $+0.00 \mathrm{e}+000$ & $\mathrm{~kg}$ \\
$Z_{\dot{v}}$ & $+0.00 \mathrm{e}+000$ & $\mathrm{~kg}$ \\
$Z_{\dot{w}}$ & $-3.55 \mathrm{e}+001$ & $\mathrm{~kg}$ \\
$Z_{\dot{p}}$ & $+0.00 \mathrm{e}+000$ & $\mathrm{~kg} \cdot \mathrm{m} / \mathrm{rad}$ \\
$Z_{\dot{q}}$ & $-1.93 \mathrm{e}+000$ & $\mathrm{~kg} \cdot \mathrm{m} / \mathrm{rad}$ \\
$Z_{\dot{r}}$ & $+0.00 \mathrm{e}+000$ & $\mathrm{~kg} \cdot \mathrm{m} / \mathrm{rad}$ \\
$K_{\dot{u}}$ & $+0.00 \mathrm{e}+000$ & $\mathrm{~kg} \cdot \mathrm{m}$ \\
$K_{\dot{v}}$ & $+0.00 \mathrm{e}+000$ & $\mathrm{~kg} \cdot \mathrm{m}$ \\
$K_{\dot{w}}$ & $+0.00 \mathrm{e}+000$ & $\mathrm{~kg} \cdot \mathrm{m}$ \\
$K_{\dot{p}}$ & $-7.04 \mathrm{e}-002$ & $\mathrm{~kg} \cdot \mathrm{m}^{2} / \mathrm{rad}$ \\
$K_{\dot{q}}$ & $+0.00 \mathrm{e}+000$ & $\mathrm{~kg} \cdot \mathrm{m}{ }^{2} / \mathrm{rad}$ \\
$K_{\dot{r}}$ & $+0.00 \mathrm{e}+000$ & $\mathrm{~kg} \cdot \mathrm{m}{ }^{2} / \mathrm{rad}$ \\
$M_{\dot{u}}$ & $+0.00 \mathrm{e}+000$ & $\mathrm{~kg} \cdot \mathrm{m}$ \\
$M_{\dot{v}}$ & $+0.00 \mathrm{e}+000$ & $\mathrm{~kg} \cdot \mathrm{m}$ \\
$M_{\dot{w}}$ & $-1.93 \mathrm{e}+000$ & $\mathrm{~kg} \cdot \mathrm{m}$ \\
$M_{\dot{p}}$ & $+0.00 \mathrm{e}+000$ & $\mathrm{~kg} \cdot \mathrm{m}{ }^{2} / \mathrm{rad}$ \\
$M_{\dot{q}}$ & $-4.88 \mathrm{e}+000$ & $\mathrm{~kg} \cdot \mathrm{m}^{2} / \mathrm{rad}$ \\
$M_{\dot{r}}$ & $+0.00 \mathrm{e}+000$ & $\mathrm{~kg} \cdot \mathrm{m}{ }^{2} / \mathrm{rad}$ \\
$N_{\dot{u}}$ & $+0.00 \mathrm{e}+000$ & $\mathrm{~kg} \cdot \mathrm{m}$ \\
$N_{\dot{v}}$ & $+1.93 \mathrm{e}+000$ & $\mathrm{~kg} \cdot \mathrm{m}$ \\
$N_{\dot{w}}$ & $+0.00 \mathrm{e}+000$ & $\mathrm{~kg} \cdot \mathrm{m}$ \\
$N_{\dot{p}}$ & $+0.00 \mathrm{e}+000$ & $\mathrm{~kg} \cdot \mathrm{m}^{2} / \mathrm{rad}$ \\
$N_{\dot{q}}$ & $+0.00 \mathrm{e}+000$ & $\mathrm{~kg} \cdot \mathrm{m} 2 / \mathrm{rad}$ \\
$N_{\dot{r}}$ & $-4.88 \mathrm{e}+000$ & $\mathrm{~kg} \cdot \mathrm{m}^{2} / \mathrm{rad}$ \\
\hline & & \\
\hline & & \\
& & \\
\hline
\end{tabular}


Table C.6: Added Mass Force Cross-term Coefficients

\begin{tabular}{ccc}
\hline Parameter & Value & Units \\
\hline$X_{u q}$ & $+0.00 \mathrm{e}+000$ & $\mathrm{~kg} / \mathrm{rad}$ \\
$X_{w q}$ & $-3.55 \mathrm{e}+001$ & $\mathrm{~kg} / \mathrm{rad}$ \\
$X_{q q}$ & $-1.93 \mathrm{e}+000$ & $\mathrm{~kg} \cdot \mathrm{m} / \mathrm{rad}$ \\
$X_{v r}$ & $+3.55 \mathrm{e}+001$ & $\mathrm{~kg} / \mathrm{rad}$ \\
$X_{r p}$ & $+0.00 \mathrm{e}+000$ & $\mathrm{~kg} \cdot \mathrm{m} / \mathrm{rad}$ \\
$X_{r r}$ & $-1.93 \mathrm{e}+000$ & $\mathrm{~kg} \cdot \mathrm{m} / \mathrm{rad}$ \\
$X_{u r}$ & $+0.00 \mathrm{e}+000$ & $\mathrm{~kg} / \mathrm{rad}$ \\
$X_{w r}$ & $+0.00 \mathrm{e}+000$ & $\mathrm{~kg} / \mathrm{rad}$ \\
$X_{v q}$ & $+0.00 \mathrm{e}+000$ & $\mathrm{~kg} / \mathrm{rad}$ \\
$X_{p q}$ & $+0.00 \mathrm{e}+000$ & $\mathrm{~kg} \cdot \mathrm{m} / \mathrm{rad}$ \\
$X_{q r}$ & $+0.00 \mathrm{e}+000$ & $\mathrm{~kg} \cdot \mathrm{m} / \mathrm{rad}$ \\
$Y_{v r}$ & $+0.00 \mathrm{e}+000$ & $\mathrm{~kg} / \mathrm{rad}$ \\
$Y_{v p}$ & $+0.00 \mathrm{e}+000$ & $\mathrm{~kg} / \mathrm{rad}$ \\
$Y_{r r a}$ & $+0.00 \mathrm{e}+000$ & $\mathrm{~kg} \cdot \mathrm{m} / \mathrm{rad}$ \\
$Y_{r p}$ & $+0.00 \mathrm{e}+000$ & $\mathrm{~kg} / \mathrm{rad}$ \\
$Y_{p p}$ & $+0.00 \mathrm{e}+000$ & $\mathrm{~kg} \cdot \mathrm{m} / \mathrm{rad}$ \\
$Y_{u p}$ & $+0.00 \mathrm{e}+000$ & $\mathrm{~kg} / \mathrm{rad}$ \\
$Y_{w r}$ & $+0.00 \mathrm{e}+000$ & $\mathrm{~kg} / \mathrm{rad}$ \\
$Y_{u r a}$ & $-9.30 \mathrm{e}-001$ & $\mathrm{~kg} / \mathrm{rad}$ \\
$Y_{w p}$ & $+3.55 \mathrm{e}+001$ & $\mathrm{~kg} / \mathrm{rad}$ \\
$Y_{p q}$ & $+1.93 \mathrm{e}+000$ & $\mathrm{~kg} \cdot \mathrm{m} / \mathrm{rad}$ \\
$Y_{q r}$ & $+0.00 \mathrm{e}+000$ & $\mathrm{~kg} \cdot \mathrm{m} / \mathrm{rad}$ \\
$Z_{w q}$ & $+0.00 \mathrm{e}+000$ & $\mathrm{~kg} / \mathrm{rad}$ \\
$Z_{u q a}$ & $+9.30 \mathrm{e}-001$ & $\mathrm{~kg} / \mathrm{rad}$ \\
$Z_{q q a}$ & $+0.00 \mathrm{e}+000$ & $\mathrm{~kg} \cdot \mathrm{m} / \mathrm{rad}$ \\
$Z_{v p}$ & $-3.55 \mathrm{e}+001$ & $\mathrm{~kg} / \mathrm{rad}$ \\
$Z_{r p}$ & $+1.93 \mathrm{e}+000$ & $\mathrm{~kg} / \mathrm{rad}$ \\
$Z_{p p}$ & $+0.00 \mathrm{e}+000$ & $\mathrm{~kg} \cdot \mathrm{m} / \mathrm{rad}$ \\
$Z_{u p}$ & $+0.00 \mathrm{e}+000$ & $\mathrm{~kg} / \mathrm{rad}$ \\
$Z_{w p}$ & $+0.00 \mathrm{e}+000$ & $\mathrm{~kg} / \mathrm{rad}$ \\
$Z_{v q}$ & $+0.00 \mathrm{e}+000$ & $\mathrm{~kg} / \mathrm{rad}$ \\
$Z_{p q}$ & $+0.00 \mathrm{e}+000$ & $\mathrm{~kg} \cdot \mathrm{m} / \mathrm{rad}$ \\
$Z_{q r}$ & $+0.00 \mathrm{e}+000$ & $\mathrm{~kg} \cdot \mathrm{m} / \mathrm{rad}$ \\
\hline \hline & &
\end{tabular}


Table C.7: Added Mass K-Moment Cross-term Coefficients

\begin{tabular}{ccc}
\hline Parameter & Value & Units \\
\hline$K_{w u}$ & $+0.00 \mathrm{e}+000$ & $\mathrm{~kg}$ \\
$K_{u q}$ & $+0.00 \mathrm{e}+000$ & $\mathrm{~kg} \cdot \mathrm{m} / \mathrm{rad}$ \\
$K_{w w}$ & $+0.00 \mathrm{e}+000$ & $\mathrm{~kg}$ \\
$K_{w q}$ & $+0.00 \mathrm{e}+000$ & $\mathrm{~kg} \cdot \mathrm{m} / \mathrm{rad}$ \\
$K_{q q}$ & $+0.00 \mathrm{e}+000$ & $\mathrm{~kg} \cdot \mathrm{m}^{2} / \mathrm{rad}^{2}$ \\
$K_{v v}$ & $+0.00 \mathrm{e}+000$ & $\mathrm{~kg}$ \\
$K_{v r}$ & $+0.00 \mathrm{e}+000$ & $\mathrm{~kg} \cdot \mathrm{m} / \mathrm{rad}$ \\
$K_{v p}$ & $+0.00 \mathrm{e}+000$ & $\mathrm{~kg} \cdot \mathrm{m} / \mathrm{rad}$ \\
$K_{r r}$ & $+0.00 \mathrm{e}+000$ & $\mathrm{~kg} \cdot \mathrm{m}^{2} / \mathrm{rad}^{2}$ \\
$K_{r p}$ & $+0.00 \mathrm{e}+000$ & $\mathrm{~kg} \cdot \mathrm{m}^{2} / \mathrm{rad}^{2}$ \\
$K_{u v}$ & $+0.00 e+000$ & $\mathrm{~kg}$ \\
$K_{v w}$ & $+0.00 e+000$ & $\mathrm{~kg}$ \\
$K_{w r}$ & $+0.00 \mathrm{e}+000$ & $\mathrm{~kg} \cdot \mathrm{m} / \mathrm{rad}$ \\
$K_{w p}$ & $+0.00 e+000$ & $\mathrm{~kg} \cdot \mathrm{m} / \mathrm{rad}$ \\
$K_{u r}$ & $+0.00 e+000$ & $\mathrm{~kg} \cdot \mathrm{m} / \mathrm{rad}$ \\
$K_{v q}$ & $+0.00 e+000$ & $\mathrm{~kg} \cdot \mathrm{m} / \mathrm{rad}^{2}$ \\
$K_{p q}$ & $+0.00 \mathrm{e}+000$ & $\mathrm{~kg} \cdot \mathrm{m}^{2} / \mathrm{rad}$ \\
$K_{q r}$ & $+0.00 \mathrm{e}+000$ & $\mathrm{~kg} \cdot \mathrm{m}^{2} / \mathrm{rad}^{2}$ \\
\hline \hline
\end{tabular}


Table C.8: Added Mass M-, N-Moment Cross-term Coefficients

\begin{tabular}{|c|c|c|}
\hline Parameter & Value & Units \\
\hline$\overline{M_{w q}}$ & $+0.00 \mathrm{e}+000$ & $\mathrm{~kg} \cdot \mathrm{m} / \mathrm{rad}$ \\
\hline$M_{u q a}$ & $+1.93 e+000$ & $\mathrm{~kg} \cdot \mathrm{m} / \mathrm{rad}$ \\
\hline$M_{u u}$ & $+0.00 e+000$ & $\mathrm{~kg}$ \\
\hline$M_{w w a}$ & $+0.00 e+000$ & $\mathrm{~kg}$ \\
\hline$M_{\text {uwa }}$ & $+3.46 e+001$ & $\mathrm{~kg}$ \\
\hline$M_{v r}$ & $+0.00 e+000$ & $\mathrm{~kg} \cdot \mathrm{m} / \mathrm{rad}$ \\
\hline$M_{v p}$ & $-1.93 e+000$ & $\mathrm{~kg} \cdot \mathrm{m} / \mathrm{rad}$ \\
\hline$M_{p p}$ & $+0.00 \mathrm{e}+000$ & $\mathrm{~kg} \cdot \mathrm{m}^{2} / \mathrm{rad}^{2}$ \\
\hline$M_{r r}$ & $+0.00 e+000$ & $\mathrm{~kg} \cdot \mathrm{m}^{2} / \mathrm{rad}^{2}$ \\
\hline$M_{r p}$ & $+4.86 e+000$ & $\mathrm{~kg} \cdot \mathrm{m}^{2} / \mathrm{rad}^{2}$ \\
\hline$M_{u v}$ & $+0.00 e+000$ & $\mathrm{~kg}$ \\
\hline$M_{v w}$ & $+0.00 \mathrm{e}+000$ & $\mathrm{~kg}$ \\
\hline$M_{u p}$ & $+0.00 \mathrm{e}+000$ & $\mathrm{~kg} \cdot \mathrm{m} / \mathrm{rad}$ \\
\hline$M_{w r}$ & $+0.00 e+000$ & $\mathrm{~kg} \cdot \mathrm{m} / \mathrm{rad}$ \\
\hline$M_{w p}$ & $+0.00 e+000$ & $\mathrm{~kg} \cdot \mathrm{m} / \mathrm{rad}$ \\
\hline$M_{u r}$ & $+0.00 e+000$ & $\mathrm{~kg} \cdot \mathrm{m} / \mathrm{rad}$ \\
\hline$M_{p q}$ & $+0.00 e+000$ & $\mathrm{~kg} \cdot \mathrm{m}^{2} / \mathrm{rad}^{2}$ \\
\hline$M_{q r}$ & $+0.00 e+000$ & $\mathrm{~kg} \cdot \mathrm{m}^{2} / \mathrm{rad}^{2}$ \\
\hline$N_{u u}$ & $+0.00 e+000$ & $\mathrm{~kg}$ \\
\hline$N_{w u}$ & $+0.00 e+000$ & $\mathrm{~kg}$ \\
\hline$N_{u q}$ & $+0.00 e+000$ & $\mathrm{~kg} \cdot \mathrm{m} / \mathrm{rad}$ \\
\hline$N_{w q}$ & $+0.00 \mathrm{e}+000$ & $\mathrm{~kg} \cdot \mathrm{m} / \mathrm{rad}$ \\
\hline$N_{q q}$ & $+0.00 e+000$ & $\mathrm{~kg} \cdot \mathrm{m}^{2} / \mathrm{rad}^{2}$ \\
\hline$N_{v v a}$ & $+0.00 \mathrm{e}+000$ & $\mathrm{~kg}$ \\
\hline$N_{v r}$ & $+0.00 \mathrm{e}+000$ & $\mathrm{~kg} \cdot \mathrm{m} / \mathrm{rad}$ \\
\hline$N_{v p}$ & $+0.00 e+000$ & $\mathrm{~kg} \cdot \mathrm{m} / \mathrm{rad}$ \\
\hline$N_{r p}$ & $+0.00 e+000$ & $\mathrm{~kg} \cdot \mathrm{m}^{2} / \mathrm{rad}^{2}$ \\
\hline$N_{p p}$ & $+0.00 \mathrm{e}+000$ & $\mathrm{~kg} \cdot \mathrm{m}^{2} / \mathrm{rad}^{2}$ \\
\hline$N_{u v a}$ & $-3.46 e+001$ & $\mathrm{~kg}$ \\
\hline$N_{v w}$ & $+0.00 \mathrm{e}+000$ & $\mathrm{~kg}$ \\
\hline$N_{u p}$ & $+0.00 \mathrm{e}+000$ & $\mathrm{~kg} \cdot \mathrm{m} / \mathrm{rad}$ \\
\hline$N_{u r a}$ & $+1.93 e+000$ & $\mathrm{~kg} \cdot \mathrm{m} / \mathrm{rad}$ \\
\hline$N_{w p}$ & $-1.93 e+000$ & $\mathrm{~kg} \cdot \mathrm{m} / \mathrm{rad}$ \\
\hline$N_{v q}$ & $+0.00 e+000$ & $\mathrm{~kg} \cdot \mathrm{m} / \mathrm{rad}$ \\
\hline$N_{p q}$ & $-4.86 e+000$ & $\mathrm{~kg} \cdot \mathrm{m}^{2} / \mathrm{rad}^{2}$ \\
\hline$N_{q r}$ & $+0.00 e+000$ & $\mathrm{~kg} \cdot \mathrm{m}^{2} / \mathrm{rad}^{2}$ \\
\hline
\end{tabular}

Table C.9: Propeller Terms

\begin{tabular}{ccc}
\hline \hline Parameter & Value & Units \\
\hline$X_{\text {prop }}$ & $+3.86 \mathrm{e}+000$ & $\mathrm{~N}$ \\
$K_{\text {prop }}$ & $-5.43 \mathrm{e}-001$ & $\mathrm{~N} \cdot \mathrm{m}$ \\
\hline \hline
\end{tabular}


Table C.10: Control Fin Coefficients

\begin{tabular}{ccc}
\hline \hline Parameter & Value & Units \\
\hline$Y_{\text {uudr }}$ & $+9.64 \mathrm{e}+000$ & $\mathrm{~kg} /(\mathrm{m} \cdot \mathrm{rad})$ \\
$Z_{\text {uuds }}$ & $-9.64 \mathrm{e}+000$ & $\mathrm{~kg} /(\mathrm{m} \cdot \mathrm{rad})$ \\
$M_{u u d s}$ & $-6.15 \mathrm{e}+000$ & $\mathrm{~kg} / \mathrm{rad}$ \\
$N_{\text {uudr }}$ & $-6.15 \mathrm{e}+000$ & $\mathrm{~kg} / \mathrm{rad}$ \\
$Y_{\text {uvf }}$ & $-9.64 \mathrm{e}+000$ & $\mathrm{~kg} / \mathrm{m}$ \\
$Z_{\text {uwf }}$ & $-9.64 \mathrm{e}+000$ & $\mathrm{~kg} / \mathrm{m}$ \\
$Y_{\text {urf }}$ & $+6.15 \mathrm{e}+000$ & $\mathrm{~kg} / \mathrm{rad}$ \\
$Z_{u q f}$ & $-6.15 \mathrm{e}+000$ & $\mathrm{~kg} / \mathrm{rad}$ \\
$M_{u w f}$ & $-6.15 \mathrm{e}+000$ & $\mathrm{~kg}$ \\
$N_{u v f}$ & $+6.15 \mathrm{e}+000$ & $\mathrm{~kg}$ \\
$M_{u q f}$ & $-3.93 \mathrm{e}+000$ & $\mathrm{~kg} \cdot \mathrm{m} / \mathrm{rad}$ \\
$N_{\text {urf }}$ & $-3.93 \mathrm{e}+000$ & $\mathrm{~kg} \cdot \mathrm{m} / \mathrm{rad}$ \\
\hline \hline
\end{tabular}




\section{Appendix D}

\section{Tables of Linearized Model Parameters}

Note that all coefficients are calculated for the STD REMUS hull profile.

Table D.1: Linearized Combined Coefficients

\begin{tabular}{cccl}
\hline \hline Parameter & Value & Units & Description \\
\hline$Z_{w c}$ & $-1.57 \mathrm{e}+001$ & $\mathrm{~kg} / \mathrm{s}$ & Crossflow Drag \\
$Z_{w l}$ & $-3.45 \mathrm{e}+001$ & $\mathrm{~kg} \cdot \mathrm{m} / \mathrm{s}$ & Body Lift \\
$Z_{w f}$ & $-1.64 \mathrm{e}+001$ & $\mathrm{~kg} \cdot \mathrm{m} / \mathrm{s}$ & Fin Lift \\
& & & \\
$Z_{q c}$ & $+1.20 \mathrm{e}-001$ & $\mathrm{~kg} \cdot \mathrm{m} / \mathrm{s}$ & Crossflow Drag \\
$Z_{q a}$ & $+1.44 \mathrm{e}+000$ & $\mathrm{~kg} \cdot \mathrm{m} / \mathrm{s}$ & Added Mass Cross Term \\
$Z_{q f}$ & $-1.12 \mathrm{e}+001$ & $\mathrm{~kg} \cdot \mathrm{m} / \mathrm{s}$ & Fin Lift \\
& & & \\
$M_{w c}$ & $-4.03 \mathrm{e}-001$ & $\mathrm{~kg} \cdot \mathrm{m} / \mathrm{s}$ & Crossflow Drag \\
$M_{w a}$ & $+5.34 \mathrm{e}+001$ & $\mathrm{~kg} \cdot \mathrm{m} / \mathrm{s}$ & Added Mass Cross Term \\
$M_{w l}$ & $-1.11 \mathrm{e}+001$ & $\mathrm{~kg} \cdot \mathrm{m} / \mathrm{s}$ & Body Lift \\
$M_{w f}$ & $-1.12 \mathrm{e}+001$ & $\mathrm{~kg} \cdot \mathrm{m} / \mathrm{s}$ & Fin Lift \\
& & & \\
$M_{q c}$ & $-2.16 \mathrm{e}+000$ & $\mathrm{~kg} \cdot \mathrm{m} 2 / \mathrm{s}$ & Crossflow Drag \\
$M_{q a}$ & $+2.97 \mathrm{e}+000$ & $\mathrm{~kg} \cdot \mathrm{m}^{2} / \mathrm{s}$ & Added Mass Cross Term \\
$M_{q f}$ & $-7.68 \mathrm{e}+000$ & $\mathrm{~kg} \cdot \mathrm{m}^{2} / \mathrm{s}$ & Fin Lift \\
\hline \hline
\end{tabular}


Table D.2: Linearized Maneuvering Coefficients

\begin{tabular}{cccl}
\hline \hline Parameter & Value & Units & Description \\
\hline$X_{\theta}$ & $+8.90 \mathrm{e}+000$ & $\mathrm{~kg} \cdot \mathrm{m} / \mathrm{s}^{2}$ & Hydrostatic \\
$X_{u}$ & $-1.35 \mathrm{e}+001$ & $\mathrm{~kg} / \mathrm{s}$ & Axial Drag \\
$X_{\dot{u}}$ & $-9.30 \mathrm{e}-001$ & $\mathrm{~kg}$ & Added Mass \\
$X_{q}$ & $-5.78 \mathrm{e}-001$ & $\mathrm{~kg} \cdot \mathrm{m} / \mathrm{s}$ & Added Mass Cross Term \\
& & & \\
$Z_{w}$ & $-6.66 \mathrm{e}+001$ & $\mathrm{~kg} / \mathrm{s}$ & Combined Term \\
$Z_{q}$ & $-9.67 \mathrm{e}+000$ & $\mathrm{~kg} \cdot \mathrm{m} / \mathrm{s}$ & Combined Term \\
$Z_{w}$ & $-3.55 \mathrm{e}+001$ & $\mathrm{~kg}$ & Added Mass \\
$Z_{\dot{q}}$ & $-1.93 \mathrm{e}+000$ & $\mathrm{~kg} \cdot \mathrm{m}$ & Added Mass \\
$Z_{\delta_{s}}$ & $-5.06 \mathrm{e}+001$ & $\mathrm{~kg} \cdot \mathrm{m} / \mathrm{s}^{2}$ & Fin Lift \\
& & & \\
$M_{\theta}$ & $-5.77 \mathrm{e}+000$ & $\mathrm{~kg} \cdot \mathrm{m}^{2} / \mathrm{s}^{2}$ & Hydrostatic \\
$M_{w}$ & $+3.07 \mathrm{e}+001$ & $\mathrm{~kg} \cdot \mathrm{m} / \mathrm{s}$ & Combined Term \\
$M_{q}$ & $-6.87 \mathrm{e}+000$ & $\mathrm{~kg} \cdot \mathrm{m}^{2} / \mathrm{s}$ & Combined Term \\
$M_{\dot{w}}$ & $-1.93 \mathrm{e}+000$ & $\mathrm{~kg} \cdot \mathrm{m}$ & Added Mass \\
$M_{\dot{q}}$ & $-4.88 \mathrm{e}+000$ & $\mathrm{~kg} \cdot \mathrm{m}^{2}$ & Added Mass \\
$Z_{\delta_{s}}$ & $-3.46 \mathrm{e}+001$ & $\mathrm{~kg} \cdot \mathrm{m}^{2} / \mathrm{s}^{2}$ & Fin Lift \\
\hline \hline
\end{tabular}




\section{Appendix E}

\section{MATLAB Code}

\section{E.1 Vehicle Simulation}

The REMUS simulator program was written using MATLAB. The first program, REMUS_SIM.m, loads the vehicle initial conditions and tracks the vehicle state. The second program, REMUS.m, calculates the new vehicle accelerations based on the vehicle state and control inputs.

\section{E.1.1 REMUS_SIM.m}

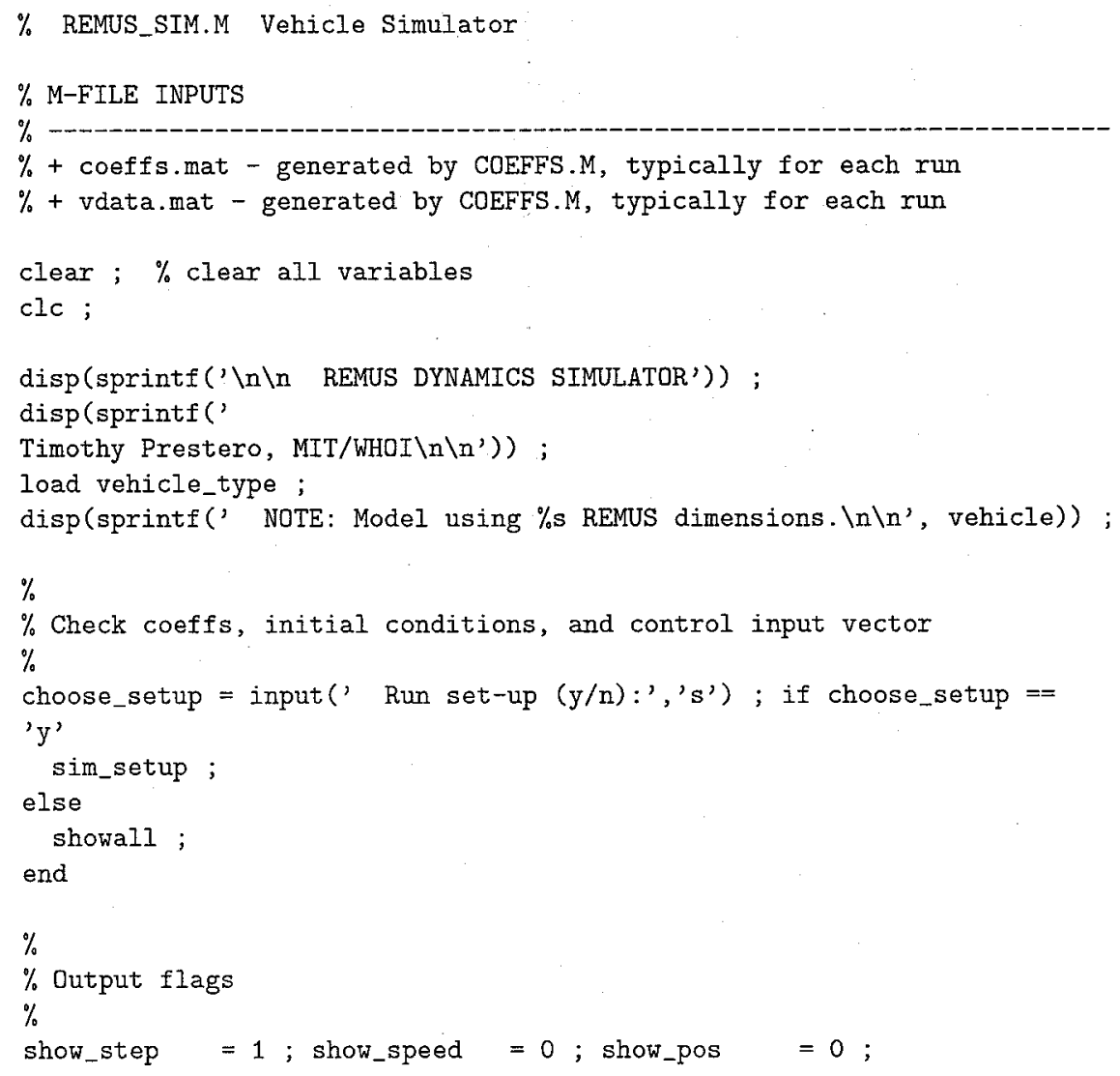




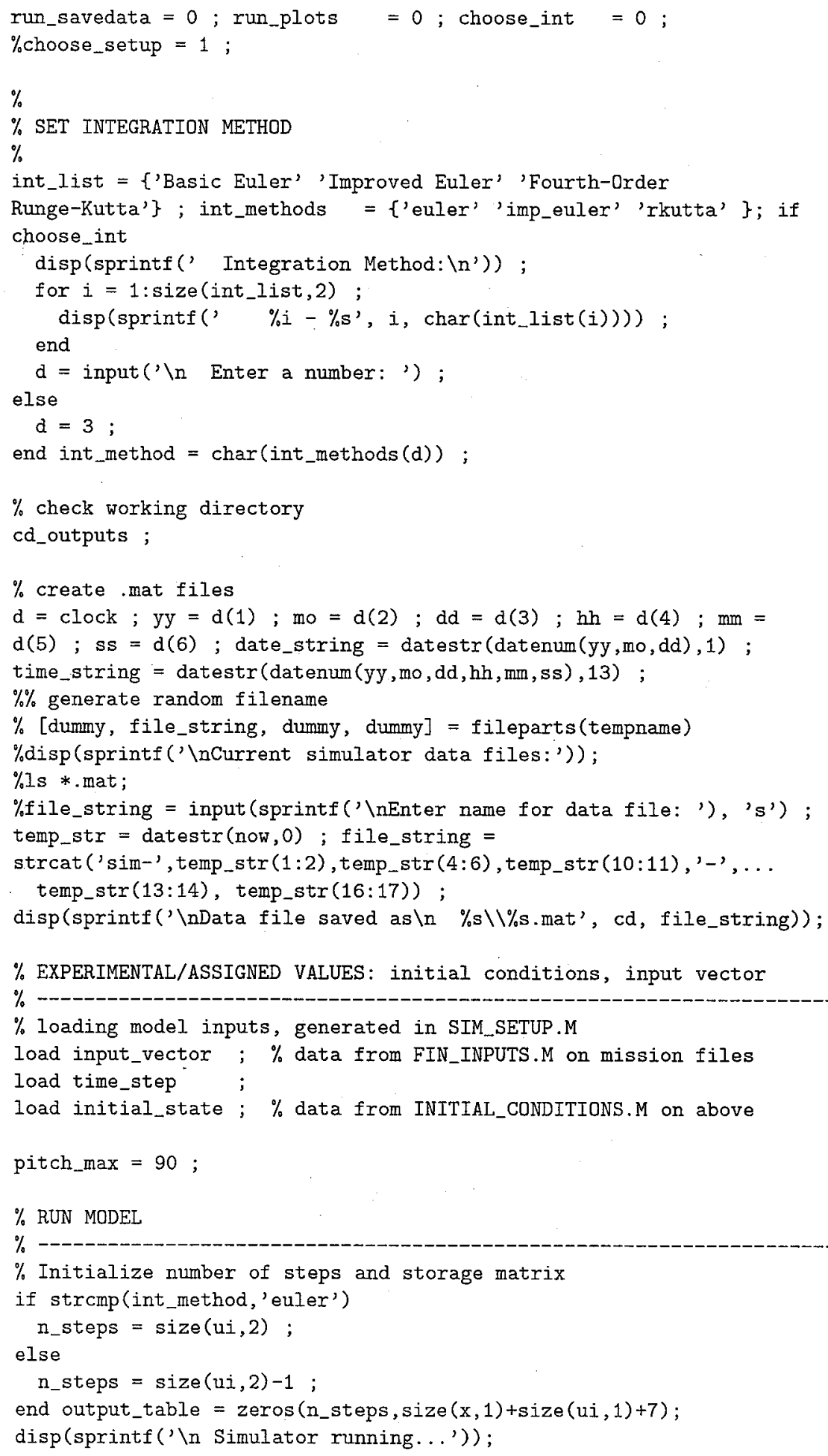




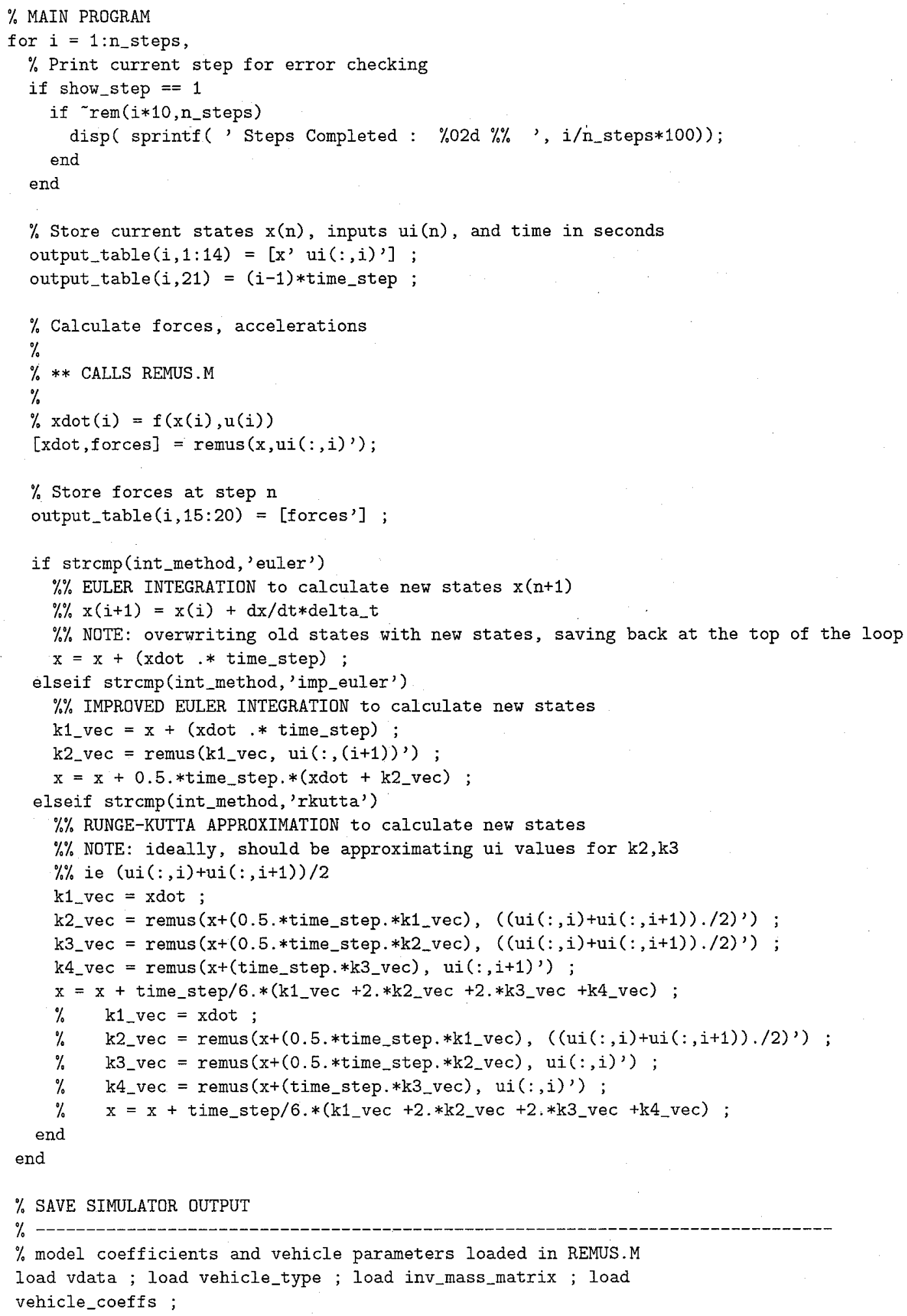




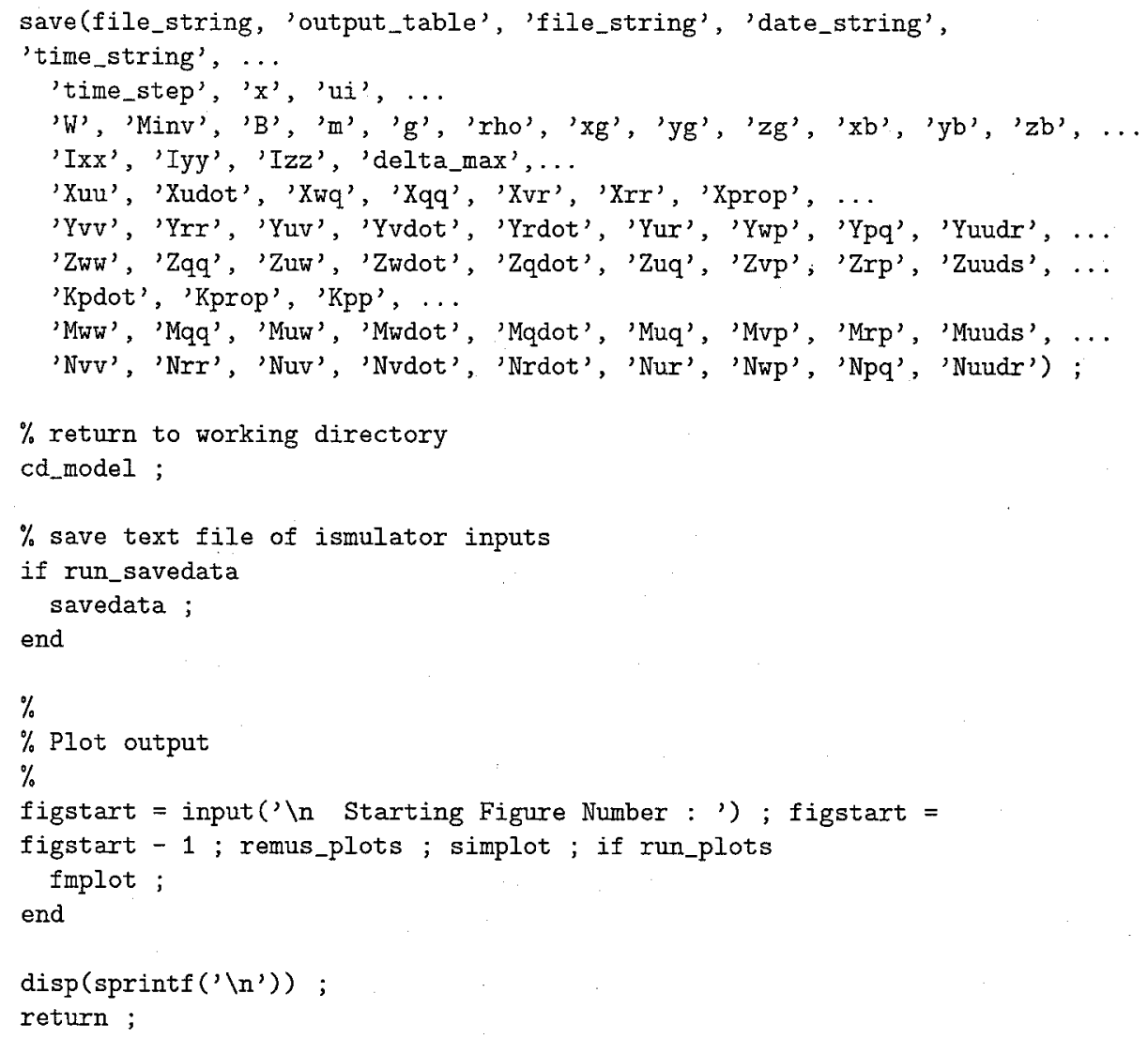

\section{E.1.2 REMUS.m}

\% REMUS.M Vehicle Simulator, returns the

$\%$ time derivative of the state vector

function [ACCELERATIONS, FORCES] = remus $(x, u i)$

$\%$ TERMS

$\%$

$\%$ STATE VECTOR:

$\% \mathrm{x}=[\mathrm{u}$ v w p q $\mathrm{r}$ xpos ypos zpos phi theta psi]'

$\%$ Body-referenced Coordinates

$\%$ u $\quad$ Surge velocity [m/sec]

$\% \mathrm{v} \quad=$ Sway velocity $\quad[\mathrm{m} / \mathrm{sec}]$

$\%$ w $\quad$ Heave velocity [m/sec]

$\% \mathrm{p} \quad=$ Roll rate $[\mathrm{rad} / \mathrm{sec}]$

$\% \mathrm{q} \quad=$ Pitch rate [rad $/ \mathrm{sec}]$

$\% \mathrm{r} \quad=$ Yaw rate [rad $/ \mathrm{sec}]$

$\%$ Earth-fixed coordinates

$\%$ xpos= Position in $\mathrm{x}$-direction [m]

$\%$ ypos $\quad=$ Position in $\mathrm{y}$-direction [m] 


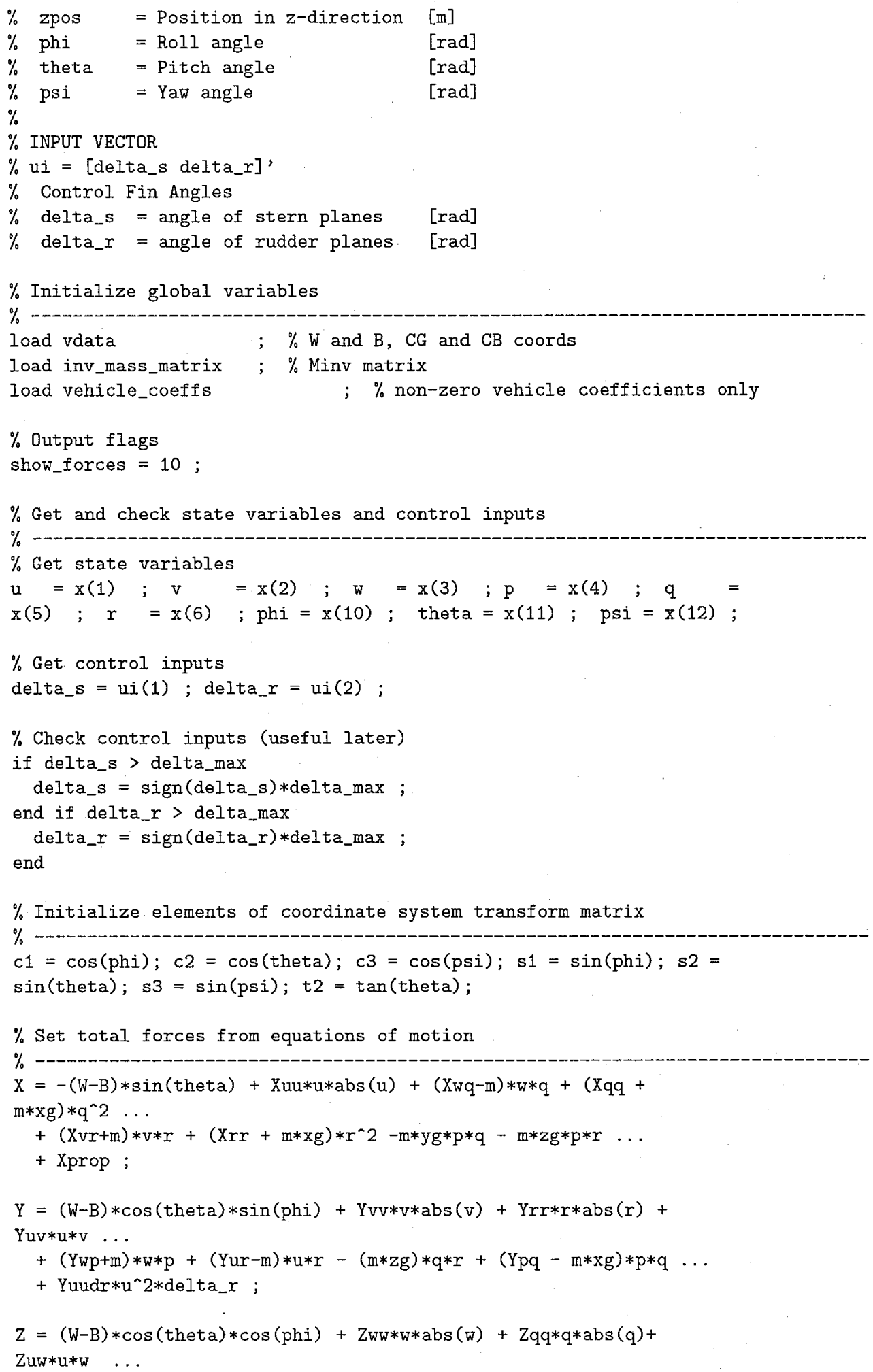




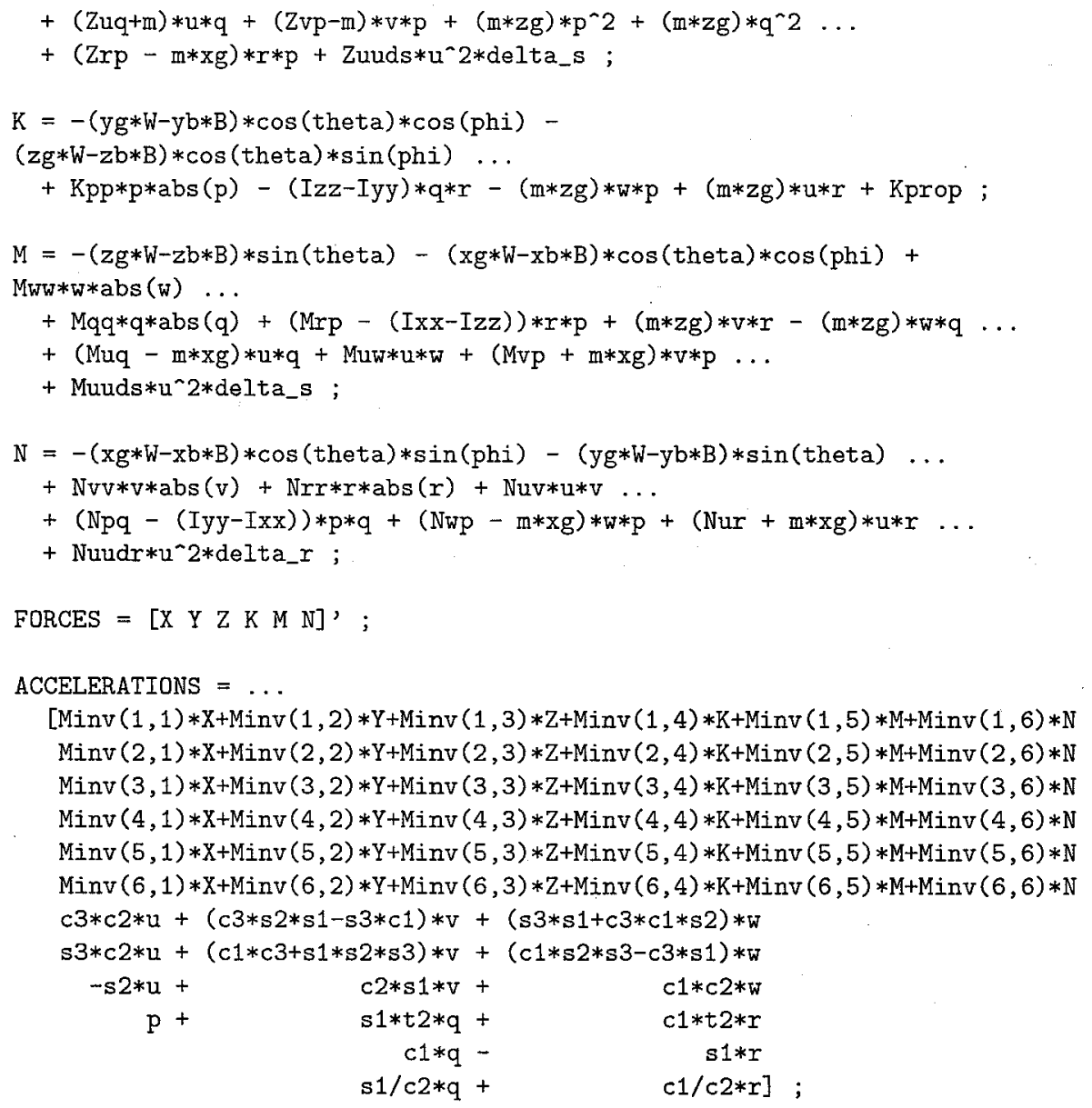




\section{Appendix $\mathrm{F}$}

\section{Example REMUS Mission File}

The following is an example REMUS mission file, taken from the thesis field experiment conducted on 27 July 1999. The goals of this particular experiment were to measure:

- the vehicle behaviour with zeroed fins

- the vehicle response to step changes in stern plane and rudder angle

- the vehicle turn radius as a function of steady-state rudder angle

- the vehicle roll offset as a function of propeller RPM

See Section 7.4.3 for the details of mission programming.

\section{F.1 REMUS Mission Code}

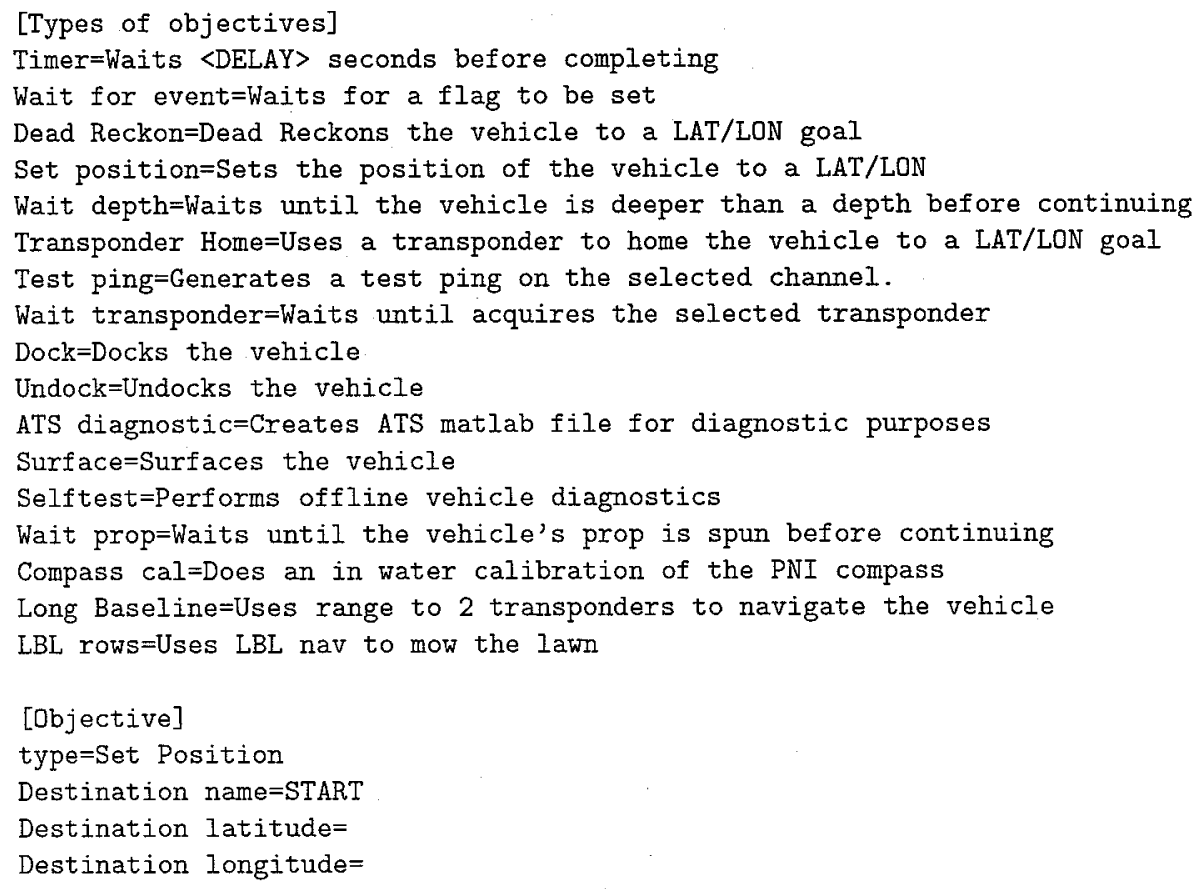




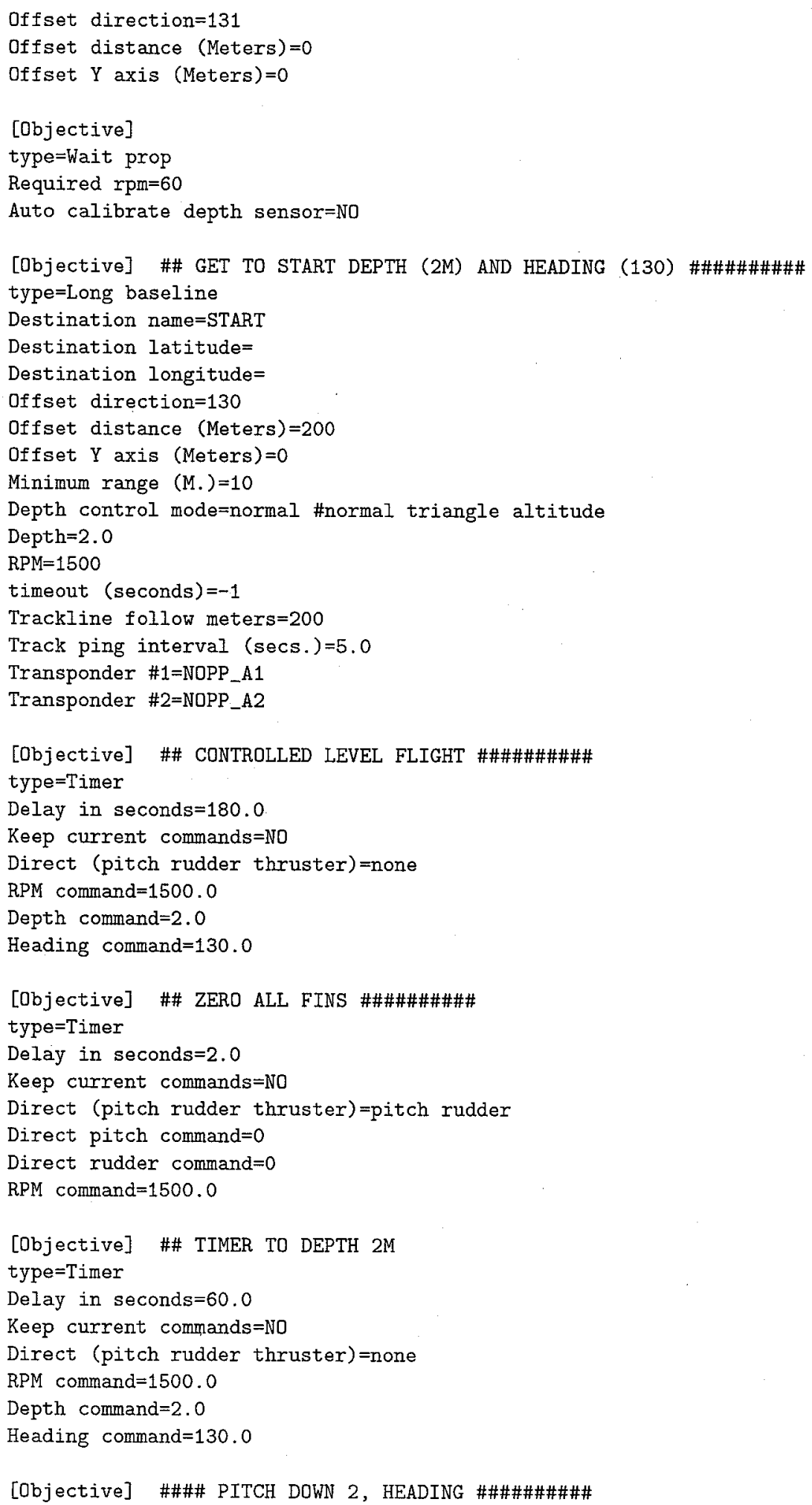




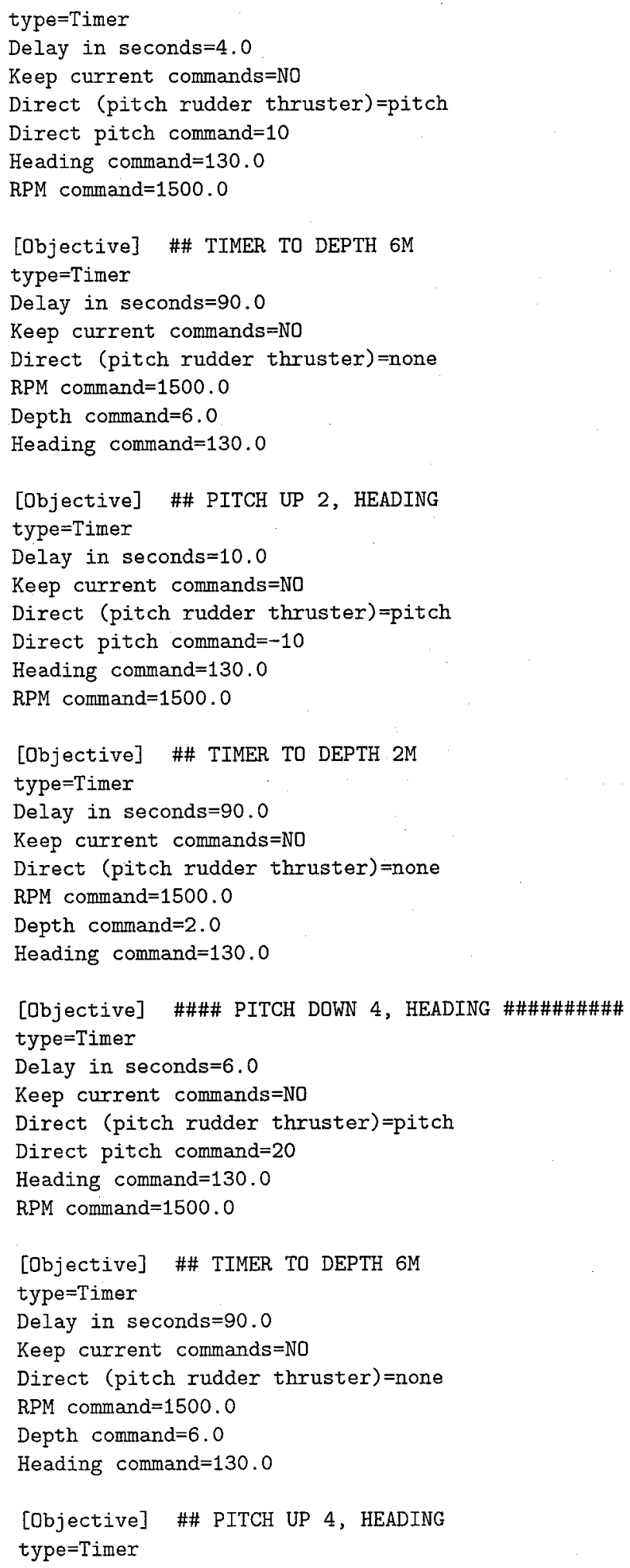


Delay in seconds $=8.0$

Keep current commands=NO

Direct (pitch rudder thruster) =pitch

Direct pitch command $=-20$

Heading command $=130.0$

RPM command $=1500.0$

[Objective] \#\# TIMER TO DEPTH 3M

type=Timer

Delay in seconds $=90.0$

Keep current commands=NO

Direct (pitch rudder thruster)=none

RPM command $=1500.0$

Depth command $=3.0$

Heading command $=130.0$

[Objective] \#\#\#\# PITCH DOWN 6, HEADING \#\#\#\#\#\#\#\#\# type=Timer

Delay in seconds $=3.0$

Keep current commands=NO

Direct (pitch rudder thruster) =pitch

Direct pitch command $=20$

Heading command $=130.0$

RPM command $=1500.0$

[Objective] \#\# TIMER TO DEPTH 6M type=Timer

Delay in seconds $=90.0$

Keep current commands=NO

Direct (pitch rudder thruster)=none

RPM command $=1500.0$

Depth command $=6.0$

Heading command $=130.0$

[Objective] \#\# PITCH UP 6, HEADING type=Timer

Delay in seconds $=4.0$

Keep current commands=NO

Direct (pitch rudder thruster)=pitch

Direct pitch command $=-20$

Heading command $=130.0$

RPM command $=1500.0$

[Objective] \#\# TIMER TO DEPTH 3M

type=Timer

Delay in seconds $=90.0$

Keep current commands=NO

Direct (pitch rudder thruster) =none

RPM command $=1500.0$

Depth command $=3.0$

Heading command $=130.0$

[Objective] \#\#\#\# RUDDER CIRCLE PORT, FIXED PITCH \#\#\#\#\#\#\#\# type=Timer

Delay in seconds $=20.0$ 


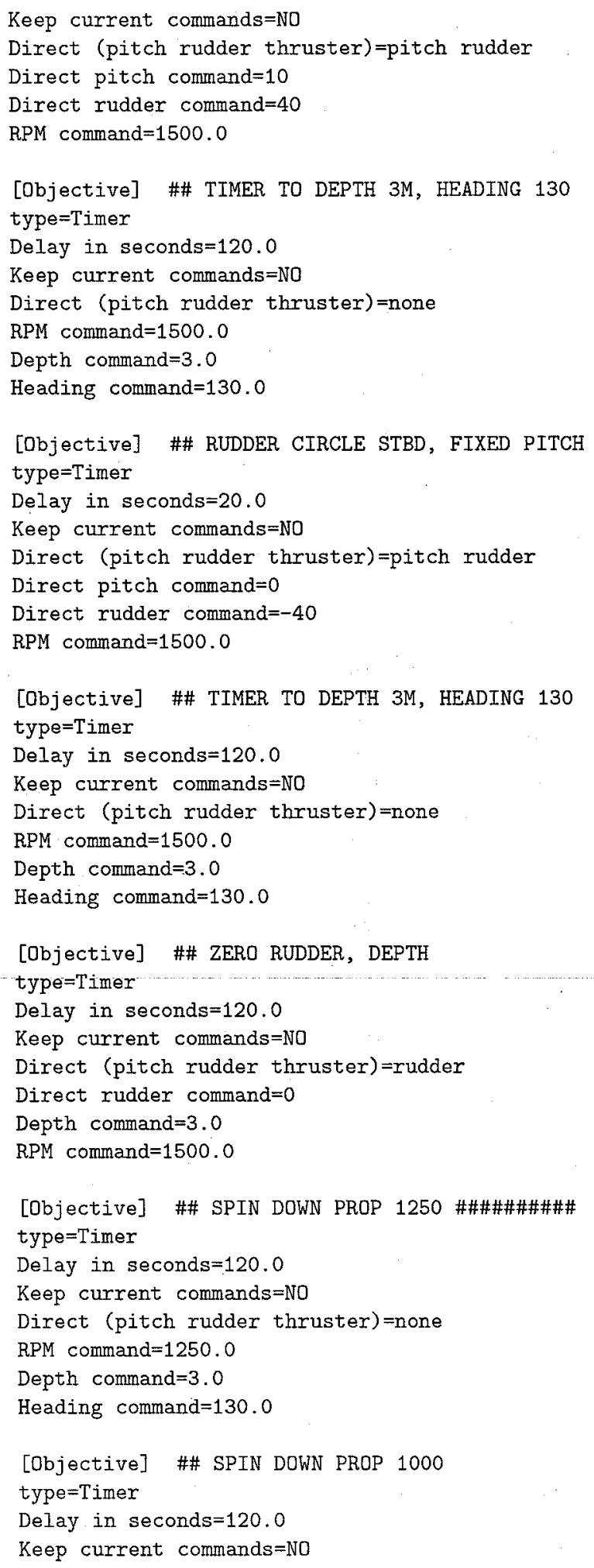


Direct (pitch rudder thruster)=none RPM command $=1000.0$

Depth command $=3.0$

Heading command $=130.0$

[Objective] \#\# SPIN DOWN PROP 750 type=Timer

Delay in seconds $=120.0$

Keep current commands $=$ NO

Direct (pitch rudder thruster)=none

RPM command $=750.0$

Depth command $=3.0$

Heading command $=130.0$

[Objective] \#\# SPIN DOWN PROP 500

type=Timer

Delay in seconds $=120.0$

Keep current commands=NO

Direct (pitch rudder thruster)=none

RPM command $=500.0$

Depth command $=3.0$

Heading command $=130.0$

[Objective]

type $=$ END 


\section{Bibliography}

[1] Martin Abkowitz. Stability and Motion Control of Ocean Vehicles. MIT Press, Cambridge, MA, 1972.

[2] B. Allen, R. Stokey, T. Austin, N. Forrester, R. Goldsborough, M. Purcell, and C. von Alt. REMUS: A small, low cost AUV; system description, field trials and performance results. In Proceedings MTS/IEEE Oceans 1997, Halifax, Canada, 1997. 37

[3] B. Allen, W. Vorus, and T. Prestero. Propulsion system performance enhancements on REMUS AUVs. In Proceedings MTS/IEEE Oceans 2000, Providence, Rhode Island, September 2000. 37

[4] P. Edgar An. An experimental self-motion study of the Ocean Explorer AUV in controlled sea states. IEEE Journal of Oceanic Engineering, 23(3):274-284, 1998. 101

[5] P. Ananthakrishnan. Dynamic response of an underwater body to surface waves. In Proceedings ASME Forum on Advances in Free Surface and Interface Fluid Dyanamics, San Francisco, CA, 1999.

[6] Robert D. Blevins. Formulas for Natural Frequency and Mode Shape. Kreiger Publishing, Florida, 1979. 28, 29

[7] M. R. Bottaccini. The stability coefficients of standard torpedoes. NAVORD Report 3346, U.S. Naval Ordnance Test Station, China Lake, CA, 1954. 25, 30, 43, 99

[8] J. Feldman. Revised standard submarine equations of motion. Report DTNSRDC/SPD-039309, David W. Taylor Naval Ship Research and Development Center, Bethesda, MD, June 1979.

[9] John E. Fidler and Charles A. Smith. Methods for predicting submersible hydrodynamic characteristics. Report NCSC TM-238-78, Naval Coastal Systems Laboratory, Panama City, FL, 1978. 30

[10] Thor I. Fossen. Guidance and Control of Ocean Vehicles. John Wiley \& Sons, New York, 1994. 27

[11] R. W. Fox and A. T. McDonald. Introduction to Fluid Mechanics. J. Wiley and Sons, New York, 4th edition, 1992.

[12] M. Gertler and G. Hagen. Standard equations of motion for submarine simulation. Report DTNSRDC 2510, David W. Taylor Naval Ship Research and Development Center, Bethesda, MD, June 1967.

[13] Michael J. Griffin. Numerical prediction of the forces and moments on submerged bodies operating near the free surface. In Proceedings of the 2000 SNAME/ASNE Student Paper Night, Massachusetts Institute of Technology, January 2000. SNAME. 101 
[14] Michael F. Hajosy. Six Degree of Freedom Vehicle Controller Design for the Operation of an Unmanned Underwater Vehicle in a Shallow Water Environment. Ocean Engineer's thesis, Massachusetts Institute of Technology, Department of Ocean Engineering, May 1994.

[15] Sighard F. Hoerner. Fluid Dynamic Drag. Published by author, 1965. 25, 26, 43

[16] Sighard F. Hoerner and Henry V. Borst. Fluid Dynamic Lift. Published by author, second edition, 1985. 30, 31, 99

[17] P. C. Hughes. Spacecraft Attitude Dynamics. John Wiley and Sons, New York, 1986. 22

[18] D. E. Humphreys. Development of the equations of motion and transfer functions for underwater vehicles. Report NCSL 287-76, Naval Coastal Systems Laboratory, Panama City, FL, July 1976.

[19] D. E. Humphreys. Dynamics and hydrodynamics of ocean vehicles. In Proceedings MTS/IEEE Oceans 2000, Providence, Rhode Island, September 2000.

[20] E. V. Lewis, editor. Principles of Naval Architecture. Society of Naval Architects and Marine Engineers, Jersey City, New Jersey, second edition, 1988. 26

[21] Woei-Min Lin and Dick Y. P. K. Yue. Numerical solutions for large-amplitude ship motions in the time domain. In Proceedings Eighteenth Symposium on Naval Hydrodynamics, Ann Arbor, Michigan, 1990. 101

[22] D. F. Myring. A theoretical study of body drag in subcritical axisymmetric flow. Aeronautical Quarterly, 27(3):186-94, August 1976. 14, 15, 43

[23] Meyer Nahon. A simplified dynamics model for autonomous underwater vehicles. In Proceedings 1996 Symposium on Autonomous Underwater Vehicle Technology, pages 373-379, June 1996. 30,99

[24] J. N. Newman. Marine Hydrodynamics. MIT Press, Massachusetts, 1977. 25, 27, 28

[25] Norman S. Nise. Control Systems Engineering. Benjamin/Cummings, San Francisco, CA, first edition, 1992. 88

[26] William D. Ramsey. Boundary Integral Methods for Lifting Bodies with Vortex Wakes. PhD dissertation, Massachusetts Institute of Technology, Department of Ocean Engineering, May 1996. 101

[27] Jeffery S. Riedel. Seaway Learning and Motion Compensation in Shallow Waters for Small $A U V s$. PhD dissertation, Naval Postgraduate School, Department of Ocean Engineering, June 1999. 101

[28] R. Stokey and T. Austin. Sequential long baseline navigation for REMUS, an autonomous underwater vehicle. In Proceedings Information Systems for Navy Divers and AUVs Operating in Very Shallow Water and Surf Zone Regions, April 1999. 37, 51

[29] Michael S. Triantafyllou. Maneuvering and control of surface and underwater vehicles. Lecture Notes for MIT Ocean Engineering Course 13.49, 1996. 25, 26

[30] C. von Alt, B. Allen, T. Austin, and R. Stokey. Remote environmental monitoring units. In Proceedings MTS/IEEE Oceans 1994, Cambridge, MA, 1994. 13, 37

[31] C. von Alt and J.F. Grassle. LEO-15: An unmanned long term environmental observatory. In Proceedings MTS/IEEE Oceans 1992, Newport, RI, 1992. 12, 37 
[32] L. F. Whicker and L. F. Fehlner. Free-stream characteristics of a family of low-aspect ratio control surfaces. Technical Report 933, David Taylor Model Basin, 1958. NC. 26

[33] Christopher J. Willy. Attitude Control of an Underwater Vehicle Subjected to Waves. Ocean Engineer's thesis, Massachusetts Institute of Technology, Department of Ocean Engineering, May 1994. 101

[34] Ming Xue. Three-dimensional fully non-linear simulation of waves and wave-body interactions. $\mathrm{PhD}$ dissertation, Massachusetts Institute of Technology, Department of Ocean Engineering, May 1997. 101

[35] D. R. Yoerger, J.G. Cooke, and J.-J. E. Slotine. The influence of thruster dynamics on underwater vehicle behavior and their incorporation into control system design. IEEE Journal of Oceanic Engineering, 15:167-178, July 1990. 33 